\title{
Anwendungsbezogene Weiterentwicklung der abbildenden Ellipsometrie
}

\author{
Dissertation
}

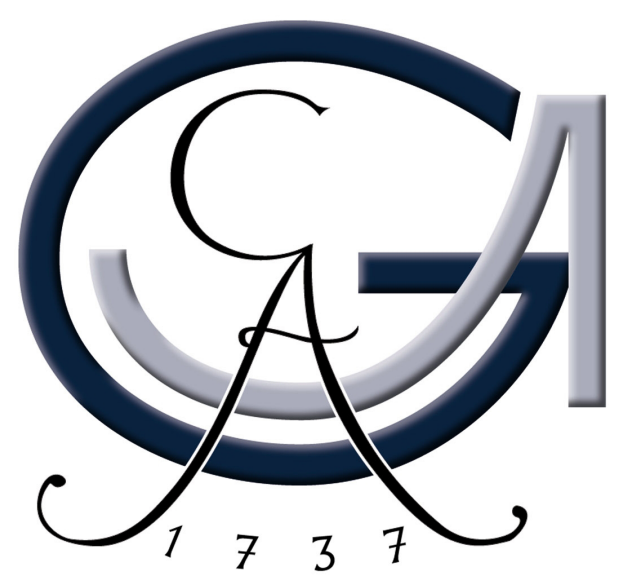

zur Erlangung des mathematisch-naturwissenschaftlichen Doktorgrades

\author{
„Doctor rerum naturalium“ \\ der Georg-August-Universität Göttingen \\ im Promotionsprogramm Chemie \\ der Georg-August-Universität Göttingen (GAUSS) \\ vorgelegt von
}

Christian Röling

Göttingen, 2017 


\section{Betreuungsausschuss}

Prof. Dr. Philipp Vana, Institut für Physikalische Chemie,

MBA Georg-August-Universität Göttingen

Prof. Dr. Andreas Janshoff Institut für Physikalische Chemie, Georg-August-Universität Göttingen

Mitglieder der Prüfungskommission

\section{Referent}

Prof. Dr. Philipp Vana, Institut für Physikalische Chemie, MBA Georg-August-Universität Göttingen

\section{Korreferent}

Prof. Dr. Andreas Janshoff Institut für Physikalische Chemie, Georg-August-Universität Göttingen

\section{Weitere Mitglieder der Prüfungskommission}

Prof. Dr. Jörg Enderlein

Prof. Dr. Jörg Schroeder

Dr. Florian Ehlers

Dr. Thomas Zeuch
III. Physikalisches Institut, Georg-August-Universität Göttingen Institut für Physikalische Chemie, Georg-August-Universität Göttingen Institut für Physikalische Chemie, Georg-August-Universität Göttingen Institut für Physikalische Chemie, Georg-August-Universität Göttingen

Tag der mündlichen Prüfung 7.9.2017 


\section{Abstract}

Since the work of Geim of graphene as the first 2D material, application and research of 2D materials is the main booming branch this decade. Overall the imaging techniques like RAMAN Imaging, AFM and optical microscopy are very unique in localizing characterizing these kind of materials, which are in the beginning only several micron large. This work is about one technique that combines the advantage of the sensitivity of an AFM and the lateral resolution of an optical microscope: Imaging Ellipsometry. This non-destructive method offers the opportunity for characterizing micro-structured thin films regarding thickness and optical properties.

How does ellipsometry and especially imaging ellipsometry work? What kind of applications can be considered for this method? What is the confinement of this technique? How could this technique can be extended for new applications? These are the main aspects of this work. As a non-destructive tool in the field of surface science, this work also show several applications ranging from micro-structured organic semi-conductive single-crystals to micro-structured polymer surfaces. Another aspects are the enhancements of the instrument regarding spectroscopy in the UV/NIR range and spectroscopic mapping for example of a micro graphene flake.

This work also shows that an imaging ellipsometer is a multi-tool in the field of surface science and can be used as a polarization microscope, brewster angle microscope or contact angle measuring instrument. 


\section{Inhaltsverzeichnis}

1 Einleitung und Zielsetzung $\quad 7$

2 Grundlagen 10

2.1 Einleitung . . . . . . . . . . . . . . . . . . . . 10

2.2 Polarisation . . . . . . . . . . . . . . . . . . . . . . . 10

2.3 Reflexion und Brechung . . . . . . . . . . . . . . . . . . . 14

2.4 Optische Interferenz _ . . . . . . . . . . . . . . . . . . . . . 19

2.5 Fundamentalgleichung . . . . . . . . . . . . . . . . . . . . . 22

2.6 Optische Konstanten . . . . . . . . . . . . . . . . . . . . . . 23

2.6.1 Isotropes Medium . . . . . . . . . . . . . . . . 23

2.6.2 Anisotropes Medium . . . . . . . . . . . . . . . . . 24

2.6 .3 Dispersion . . . . . . . . . . . . . . . . . 25

2.6.4 Dielektrische Funktionsmodelle . . . . . . . . . . . . . 27

2.6.4.1 Lorentz . . . . . . . . . . . . . . . . 27

2.6.4.2 Sellmeier und Cauchy . . . . . . . . . . . 30

2.6.4.3 Urbach . . . . . . . . . . . . . . . . . 33

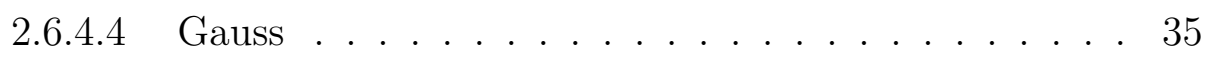

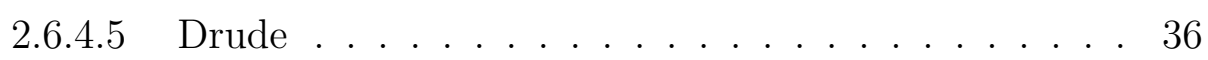

2.6.4.6 Tauc-Lorentz, Tauc-Lorentz-Urbach und Cody-Lorentz-

Urbach . . . . . . . . . . . . . 37

2.6.5 Effektives Medium Theorie . . . . . . . . . . . . . 39

2.6.5.1 Sphärische Mikrostruktur . . . . . . . . . . . . 39

2.6.5.2 Laminare Mikrostruktur . . . . . . . . . . . . 40

3 Methoden $\quad \mathbf{4 2}$

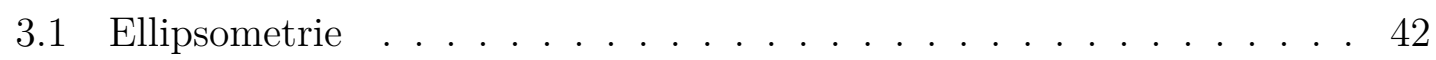

3.1.1 Messmodi ..................... 42

3.1.1.1 Einwellenellipsometrie . . . . . . . . . . . . 42

3.1.1.2 Spektroskopische Ellipsometrie . . . . . . . . . 44

3.1.2 Ellipsometriearten ................... . . 46

3.1.2.1 Nulling Ellipsometrie . . . . . . . . . . . . . . . . 46

3.1.3 Abbildende Nulling Ellipsometrie . . . . . . . . . . . . . . . . 48

3.1.3.1 Historie . . . . . . . . . . . . . . . . . . . 48

3.1.3.2 Ellipsometric Platform (EP) . . . . . . . . . . . 49

3.1.3.3 Software ......................... 55

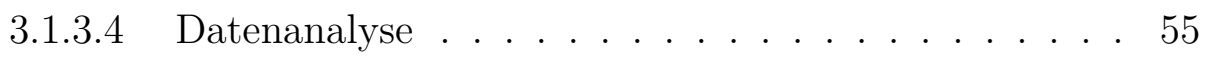

3.2 Zusätzliche Messmethoden . . . . . . . . . . . . . . . . 57

3.2 .1 ATR-FTIR-Spektroskopie . . . . . . . . . . 57

3.2.1.1 Infrarot-Spektroskopie . . . . . . . . . . 57 
3.2.1.2 Fourier-Transform-IR-Spektroskopie . . . . . . . 57

3.2.1.3 Attenuated Total Reflection FTIR-Spektroskopie . . 58

3.2.2 Rasterkraftmikroskopie . . . . . . . . . . . . . . 60

3.2.2.1 Messprinzip und Aufbau . . . . . . . . . . . 60

3.2 .3 Kontaktwinkel . . . . . . . . . . . . . . . . . 62

3.2.3.1 Messprinzip und Aufbau . . . . . . . . . . . 62

4 Ergebnisse

4.1 Imaging . . . . . . . . . . . . . . . . . . . . . . 64

4.1 .1 Quantitative Analyse . . . . . . . . . . . . . 64

4.1.1.1 Micro-contact Printing . . . . . . . . . . . . 64

4.1.1.2 Laserablation . . . . . . . . . . . . . . 67

4.1.1.3 Mikroprägelithografie . . . . . . . . . . . . . 69

4.1.1.4 $\mathrm{As}_{2} \mathrm{~S}_{3} \ldots \ldots \ldots \ldots \ldots \ldots . \ldots \ldots 71$

4.1.1.5 Mikrokristalle............... 75

4.1.1.6 Einfluss des Objektives auf die ellipsometrischen Messwerte bei Maps . . . . . . . . . . . . . . . . 82

4.1 .2 Ultra-Objektiv . . . . . . . . . . . . . . . . . 85

4.1 .3 Beamcutter . . . . . . . . . . . . . . 88

4.1.3.1 PET . . . . . . . . . . . . . 89

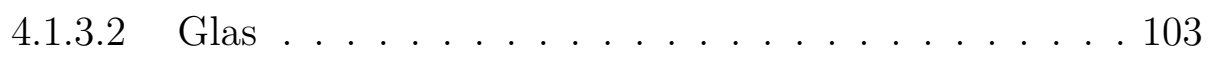

4.1.4 Kontaktwinkelmessungen . . . . . . . . . . . . . . . 108

4.2 Spektroskopie . . . . . . . . . . . . . . . . . . . . . 110

4.2.1 Monochromator . . . . . . . . . . . . . . . . 110

4.2 .2 UV/NIR-Erweiterung . . . . . . . . . . . . . . . . . . . . . . . . . . . . 125

4.2 .3 Spektroskopisches Mapping . . . . . . . . . . . . . 128

4.3 Messablauf . . . . . . . . . . . . . . . . . . . . . . . . . . 131

4.3.1 Kompensator . . . . . . . . . . . . . . . 131

4.3 .2 Fit . . . . . . . . . . . . . . . . . 132

4.4 Zellen . . . . . . . . . . . . . . . . . . 136

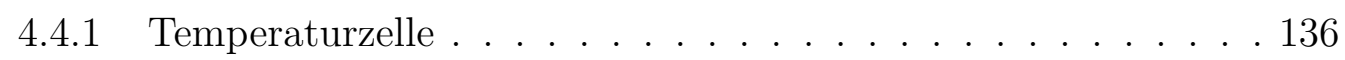

4.4 Light-guides . . . . . . . . . . . . . . . . . 138

5 Zusammenfassung und Ausblick $\quad 143$

6 Probenpräparation $\quad \mathbf{1 4 5}$

6.1 Selbst hergestellte Proben . . . . . . . . . . . . . . . . . . 145

6.1.1 Hydrophilisierung . . . . . . . . . . . . . . . . . . . . . . . . . . . . . . . . . . . . . . . . .

6.1 .2 Spin-Coating . . . . . . . . . . . . . . . . . . . . . . . . . . . . . . . . . . . .

6.1.3 Mikrokontakt Printing . . . . . . . . . . . . . 145

6.2 Kooperation . . . . . . . . . . . . . . . . . . . 146

6.2.1 Laserablation . . . . . . . . . . . . . . . . . 146

6.2.2 Mikroprägelithographie von PET . . . . . . . . . . . . . . 146

$6.2 .3 \quad \mathrm{As}_{2} \mathrm{~S}_{3} \ldots \ldots \ldots \ldots \ldots \ldots$

6.2 .4 Mikrokristalle . . . . . . . . . . . . . . . . 146

6.2 .5 PET-Folie . . . . . . . . . . . . . . . . . . . . . . . . . . . . . . . . . . . . . . .

6.2.6 Graphen . . . . . . . . . . . . . . . . . 147 
6.2.7 Plastik Solar Zellen . . . . . . . . . . . . . . . . . . . . 147

7 Appendix $\quad 148$

$\begin{array}{ll}\text { Tabellenverzeichnis } & 154\end{array}$

$\begin{array}{ll}\text { Literaturverzeichnis } & 154\end{array}$ 


\section{Einleitung und Zielsetzung}

Tausende Naturwissenschaftler weltweit streben nach der höchsten aller wissenschaftlichen Auszeichungen: dem Nobelpreis. Im Jahr 2010 erhielten Andre Geim und Kostya Novoselov diese Ehrung für ihre herausragenden Arbeiten an Graphen. Das 2D-Material zeichnet sich neben einer hohen elektrischen Leitfähigkeit durch Flexibilität und Transparenz aus und scheint dadurch bestens geeignet für Anwendungen im Bereich der Optoelektronik [18]. Eine Monolage Graphen wird in der Literatur als dünnste atomare Schicht bezeichnet und wurde von Geim und Novoselov mittels Rasterkraftmikroskopie (atomic force microscopy AFM), Transmissionselektronenmikroskopie (transmission electron microscopy TEM) und Rasterelektronenmikroskopie (scanning electron microscopy SEM) visualisiert [25]. Zusätzlich zu den abbildenden Messmethoden wie AFM, TEM und SEM wurden ebenfalls spektroskopische Verfahren wie RAMAN-Spektroskopie und spektroskopische Ellipsometrie zur Charakterisierung der optischen Eigenschaften von Graphen eingesetzt [25, 59].

Aufgrund von Größe und Eigenschaften der Graphenflocken (Durchmesser etwa 15 $\mu \mathrm{m}$, Schichtdicke etwa $0.4 \mathrm{~nm}$ ) wurden darüber hinaus Studien mittels abbildender Ellipsometrie spektroskopisch durchgeführt. Nach der Lokalisierung der dünnsten atomaren Schichten konnten Graphen Monolagen spektroskopisch untersucht und mittels Maps visualisiert werden [2, 62]. Neben Graphen und anderen Anwendungsgebieten der klassischen Ellipsometrie wie Plastic solar cell, Halbleiter oder optischen Coatings wird die abbildende Ellipsometrie meist bei sehr dünnen und mikro-strukturierten Proben eingesetzt $[4,45]$. Die abbildende Ellipsometrie ${ }^{1}$ ist im Vergleich zur klassischen Ellipsometrie ${ }^{2}$ eine relativ junge Messtechnik.

In dieser Arbeit steht die abbildende Ellipsometrie, deren Weiterentwicklung und Anwendung als zentrale Messtechnik von nanoskaligen Materialien an planaren Oberflächen im Fokus. An geeigneten Beispielen werden die Vorteile der abbildenden Ellipsometrie, wie Mapping oder Auswahl der Messbereiche, gezeigt. Neben einer detaillierten Beschreibung der Messtechnik wird an geeigneten Probensystemen aufgezeigt, welche Schlussfolgerungen sich anhand der erhobenen Daten bezüglich der Proben ableiten lassen. Zusätzlich soll die angefertigte Arbeit zukünftigen Anwendern im Bereich der Oberflächenanalytik als Leitfaden dienen.

Neben der abbildenden Ellipsometrie werden auch anderen Messtechniken zur Oberflächencharakterisierung - wie FTIR-ATR-Spektroskopie, Kontaktwinkelmessungen und Rasterkraftmikroskopie - verwendet. Die Dissertation wurde in Kooperation mit der Firma Accurion GmbH im Zeitraum von 2009 bis 2015 angefertigt. Neben einer Dokumentation der Weiterentwicklung neuer Anwendungsbereiche - wie

\footnotetext{
${ }^{1}$ nahezu 30 Jahre $[32,9]$

${ }^{2}$ fast 130 Jahre [16]
} 
beispielsweise Kontaktwinkelmessungen mittels abbildendem Ellipsometer - werden Neuerungen und Verbesserungen von Hard- und Software ebenfalls durch geeignete Probensysteme verdeutlicht.

Zusätzlich zu den klassischen Einschichtsystemen bekannter Polymere (wie PS und PMMA) wurden neue polymere Materialien im Bereich der organischen Solarzellen spektrokopisch untersucht, da insbesondere dieses Anwendungsgebiet im letzten Jahrzehnt von besonderem Interesse für die Industrie zu sein scheint.

Ziel dieser angefertigten Arbeit war es die enormen Vorteile, die diese Messtechnik im Vergleich zu nicht bildgebenden System bietet, herauszuarbeiten und an geeigneten Fallbeispielen zu verdeutlichen. Aufgrund der Komplexität der Messtechnik, sowie der physikalischen Grundlagen an sich, sind Anwender häufig geneigt andere Messtechniken für die Charakterisierung ihrer Oberflächen zu nutzen. Im Bereich der Oberflächenanalytik an planaren Flächen werden neben AFM und Kontaktwinkelmessungen Messverfahren wie optische Mikroskopie, Polarisationsmikroskopie, Floureszenzmikroskopie, IR-Mikroskopie, RAMAN-Imaging, Reflektometrie, XPS und Ellipsometrie genutzt. So wird häufig stets mehr als nur eine der Methoden genutzt um Schichten an Oberflächen zu charakterisieren. Die abbildende Ellipsometrie, eine allgemein anwendbare kontaktfreie Technik, kann man unter anderem als eine der elementarsten Methoden ansehen, die dem Wissenschaftler zur Charaketerisierung planarer Oberflächen zur Verfügung steht. Denn besonders im Zeitalter der Miniaturisierung von Sensoren und Chips ist die Bildgebung des Systems bei wissenschaftlichen Studien essentiell. Häufig findet diese Methode jedoch in der Literatur nur eine untergeordnete Rolle im Vergleich zu anderen Ellipsometriearten. Aufgrund der derzeitig geringen Beachtung der Messmethode, soll diese Arbeit dem Anwender einen fundierten Beitrag, die Vor- und Nachteile sowie die Anwendbarkeit der Messmethode an sich, verdeutlichen.

Wie bereits in Kapitel 3.1.3.3 erwähnt, entwickelte sich während der Anfertigung der Arbeit die neue Gerätegeneration Ep4, die neben einer neuen Benutzeroberfläche ebenfalls neue Messmöglichkeiten bietet, die an den Fallbeispielen aufgezeigt werden. Die Ergebnisse zeigen die 4 großen Neuerungen der Ep4 im Vergleich zur vorherigen Version des bildgebenden Ellipsometers Ep3, die in den Unterkapiteln Imaging (Kapitel 4.1) , Spektroskopie (Kapitel 4.2), Messablauf (Kapitel 4.3) und Zellen (Kapitel 4.4) aufgeteilt sind.

Das USP der Imaging Ellipsometrie, die Bildgebung, wird für quantitative Analysen (Kapitel 4.1.1) mikrostruktierter Proben genutzt. Aufgrund der ellipsometrischen lateralen Auflösung des System konnten ebenfalls erstmals Schichten auf dünnen, anisotropen, transparenten Substraten vermessen werden (Kapitel 4.1.3.1). Auch weitere bildgebende Verfahren wie Brewsterwinkelmikroskopie (Kapitel 4.1.2) und Kontaktwinkelmessungen (Kapitel 4.1.4) sollten mit dem System getesten werden und das Anwendungsfeld des Systems abrunden.

Die Erweiterungen des Wellenlängenbereichs (Kapitel 4.2.2) und Wechsel zum Monochromator (Kapitel 4.2.1) mit durchstimmbarer Wellenlänge wurden erfolgreich von der Forschung und Entwicklung umgesetzt. In dieser Arbeit sollten die Neuerungen, wie das spektroskopsiche Mapping (Kapitel 4.2.3), für den Anwender aufgezeigt und verifiziert werden. 
Im Verlauf dieser Arbeit wurde ebenfalls erreicht den hohen Zeitaufwand der Datenaufnahme durch geeignete Einstellungen und Messabläufe (Kapitel 4.3) zu minimieren.

Zusätzlich sollten neben den Messungen unter konstanten Bedingungen an der Festkörperoberfläche unter Ätmosphäre Untersuchungen von dünnen Schichten an der flüssig-flüssig Grenzfläche (Kapitel 4.4.2) und temperaturabhängige Messungen an Polymeren (Kapitel 4.4.1) durchgeführt werden. 


\section{Grundlagen}

Im ersten Kapitel werden die physikalischen Phänomene wie Interferenz, Reflexion, Absorption, Dispersion und Polarisation von Licht beschrieben, die die Grundlagen der Ellipsometrie bilden.

\subsection{Einleitung}

Der Name Ellipsometrie beruht etymologisch auf der Verwendung von elliptisch polarisiertem Licht. Ellipsometrie ist eine optische, zerstörungsfreie Messtechnik, bei der durch Bestimmung der Änderung des Polarisationsgrades des Lichts, die aufgrund von Reflexion an einer Grenzfläche auftritt, physikalische Eigenschaften (dielektrische Parameter, Schichtdicke) dünner Schichten und Materialien indirekt bestimmt werden können[22, 1].

Namenhafte Naturwissenschaftler wie Brewster, Boyle und Newton beschäftigten sich mit dem Phänomen der Wechselwirkung zwischen Licht und Materie[56]. Ihre Beobachtung von Lichtbrechung sowie Interferenz des Lichtes bei Reflexion an verschiedenen Grenzflächen stellen - gemeinsam mit der Entdeckung von Malus, der partiellen linearen Polarisation bei Reflexion von Licht [41] - die physikalischen Grundlagen der Ellipsometrie dar. Als erster Naturwissenschaftler nutzte der deutsche Physiker Paul Drude den Effekt der Phasendifferenz zwischen zwei senkrecht zueinander polarisierten Lichtstrahlen, um die Dicke eines dünnen Films zu bestimmen[16].

\subsection{Polarisation}

Licht ist eine elektromagnetische Welle ${ }^{3}$ und alle für die Ellipsometrie relevanten Eigenschaften von Licht werden durch die Maxwellschen Gleichungen ausgedrückt. In den Maxwellschen Gleichungen ist das elektrische Feld $E$ orthogonal zum magnetischen Feld $B$ und der Ausbreitungsrichtung des Lichtes in z-Richtung. In dieser Arbeit wird lediglich das Licht hinsichtlich des elektrischen Feldes betrachtet und das magnetische Feld vernachlässigt bzw. gleich null gesetzt. Photonen, als Elementarteilchen von Licht, zeigen bezüglich des elektrischen Feldes eine Orientierung im Verhältnis zur Ausbreitungsrichtung. Bei unpolarisiertem Licht sind die elektrischen Felder der aufeinander folgenden Photonen statistisch verteilt und zeigen in unterschiedliche Richtungen. Bei polarisiertem Licht ist das elektrische Feld $E$ der Gesamtheit der Photonen in eine bestimmte Richtung orientiert.

\footnotetext{
${ }^{3}$ Welle-Teilchen-Dualismus
} 
Die Wellengleichung kann durch die Kombination zweier senkrecht aufeinander stehenden, linearen Einzelwellen mit gleicher Ausbreitungsrichtung (z-Achse) beschrieben werden. Die Wellengleichung für monochromatisches ${ }^{4}$ Licht, das sich entlang der z-Achse ausbreitet, sieht im kartesischen Koordinatensystem ${ }^{5}$ wie folgt aus:

$$
\vec{E}(z, t)=\left[\begin{array}{c}
E_{\mathrm{x}} \\
E_{\mathrm{y}}
\end{array}\right] \exp \left(i\left(k_{\mathrm{z}}-\omega t\right)\right.
$$

mit

$$
\begin{aligned}
& E_{\mathrm{x}}=E_{\mathrm{x} 0} \exp \left(i \delta_{\mathrm{x}}\right) \\
& E_{\mathrm{y}}=E_{\mathrm{y} 0} \exp \left(i \delta_{\mathrm{y}}\right) \\
& k_{\mathrm{z}}=\frac{\omega}{c} \tilde{N}=\frac{\omega}{c}(n-i k)
\end{aligned}
$$

Dabei sind $E_{\mathrm{x}}$ und $E_{\mathrm{y}}$ die komplexen Amplituden des elektrischen Feldes entlang der x- bzw. y-Achse (dargestellt als Jones-Vektor), $\omega$ die Kreisfrequenz, $c$ die Lichtgeschwindigkeit im Vakuum, $\delta$ die Phase, $t$ die Zeit, $z$ die Position, $\tilde{N}$ der komplexe Brechungsindex, $n$ der Brechungsindex und $k$ der Absorptionskoeffizient des Mediums. Da ausschließlich der phasenabhängige Teil genügt, um den Zustand der Polarisation zu beschreiben, wird häufig der zeitabhängige Teil unbeachtet gelassen und einfachheitshalber in Form eines Vektors dargestellt. Häufig findet sich in der einschlägigen Literatur der Jones-Formalismus, der in dieser Arbeit mit aufgeführt wird. Für einen umfassenden Überblick sei auf die Bücher „Ellipsometry and polarized light" von R.M.A Azzam und N.M. Bashara [7] "Spectroscopic ellipsometry" von H. Fujiwara [22] sowie "Handbook of ellipsometry" von H. Tompkins und E. A. Irene[55] verwiesen.

Die Amplitude und Phase der Einzelwellen bestimmen den jeweiligen Polarisationszustand, um den es sich handelt.

Man unterscheidet zwischen drei Zuständen der Polarisation des Lichtes: linear, zirkular und ellipstisch. Die zugehörigen Jones-Vektor ${ }^{6}$ werden in Tabelle 2.1 dargestellt.

\footnotetext{
${ }^{4}$ Strahlung mit definierter Wellenlänge

${ }^{5}$ Später spricht man in einem $s$ - $p$-Koordinatensystem von $s$ - bzw. $p$-polarisiertem Licht.

${ }^{6}$ Jones-Formalismus

Diese Art der Darstellung beschreibt Licht als ebene elektromagnetische Welle in Form der Vektordarstellung, bei der die Raum- und Zeitabhängigkeit der Amplitude vernachlässigt wird. Zusätzlich werden optische Effekte bei Nichtlinearität mittels 2x2-Matrizen dargestellt und auf den Jones-Vektor angewendet.
} 


\title{
Linear
}

Die beiden senkrecht zueinander stehenden Wellen besitzen die gleiche Frequenz und schwingen in Phase. Besitzen die beiden Einzelwellen ebenfalls die gleiche Amplitude (Amplitudenverhältnis $=1$ ), ist das resultierende elektrische Feld stets um $45^{\circ} \mathrm{zu}$ den beiden Einzelwellen versetzt (Abbildung 2.1).

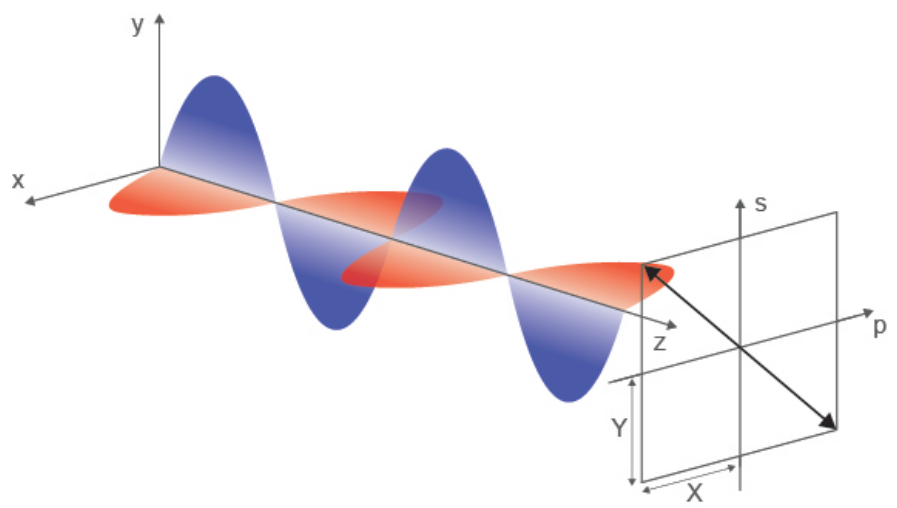

\begin{abstract}
Abbildung 2.1 Darstellung von linear polarisiertem Licht durch Kombination zweier Teilwellen gleicher Frequenz und phasengleicher Amplitude mit Projektion des zeitlichen Verlaufs des resultierenden elektrischen Feldvektors $(\mathrm{z}=0)[22,51]$.
\end{abstract}

\section{Zirkular}

Die Teilwellen haben, wie im vorherigen Fall, die gleiche Frequenz und Amplitude. Im Gegensatz zu linear polarisiertem Licht sind die Teilwellen um eine Viertelwellenlänge zueinander verschoben, was einen Phasenshift $\Delta$ von $90^{\circ}$ entspricht (Abbildung 2.2). Man unterscheidet zwischen rechts- $\left(\Delta=+90^{\circ}\right)$ und links-drehendem $\left(\Delta=-90^{\circ}\right)$ zirkular polarisiertem Licht.

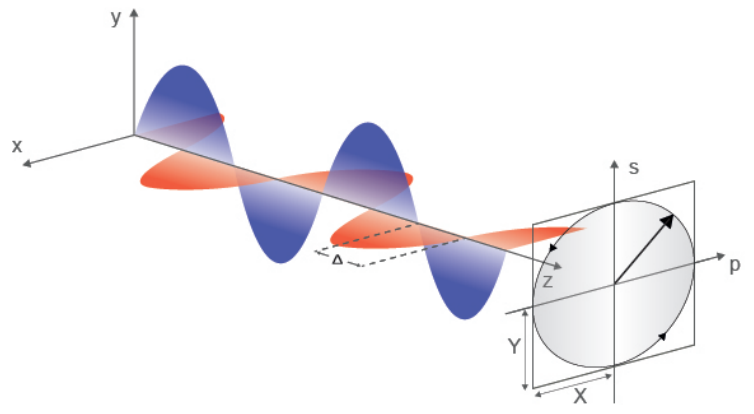

Abbildung 2.2 Darstellung von zirkular polarisiertem Licht durch die Kombination zweier Teilwellen gleicher Frequenz und Amplitude mit einer Phasenverschiebung von $90^{\circ}$ sowie Projektion des zeitlichen Verlaufs der Zirkulation des resultierenden elektrischen Feldvektors $(\mathrm{z}=0)[22,51]$. 


\section{Elliptisch}

Durch die Kombination zweier Wellen, die zwar die gleiche Frequenz besitzen, jedoch außer Phase sind, resultiert elliptisch polarisiertes Licht (Abbildung 2.3). Bei positivem Phasenshift rotiert der elektrische Feldvektor im Uhrzeigersinn, bei negativem gegen den Uhrzeigersinn.

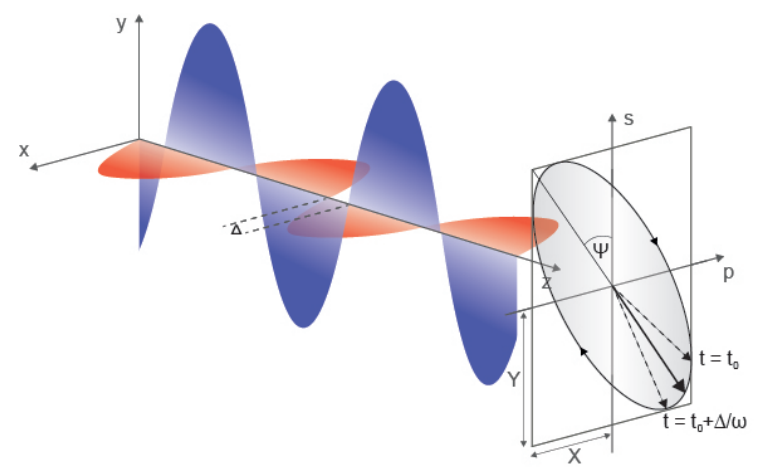

Abbildung 2.3 Darstellung von elliptisch polarisiertem Licht durch die Kombination zweier Teilwellen gleicher Frequenz und Projektion des zeitlichen Verlaufs der Polarisationsellipse mit den elliptischen Parametern Delta und Psi $(\mathrm{z}=0)[22,55,7]$.

Tabelle 2.1 Jones-Vektoren der Polarisationszustände [55, 46].

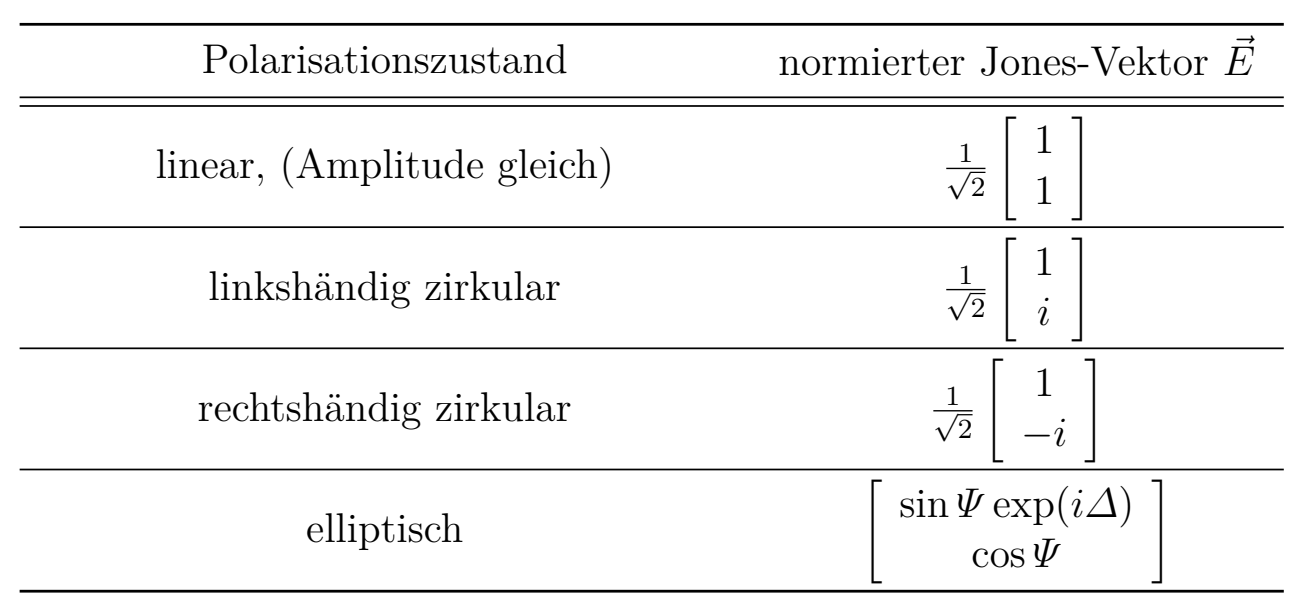

Wie in Abbildung 2.3 ersichtlich, bestimmen sowohl der Phasenshift $\Delta$ als auch die Amplituden X und Y die Ellipsenform. Das relative Verhältnis der Amplituden wird durch den Winkel $\Psi$ ausgedrückt. Es gilt:

$$
\begin{aligned}
& \Delta=\delta_{\mathrm{x}}-\delta_{\mathrm{y}} \\
& \tan \Psi=\frac{X}{Y}
\end{aligned}
$$




\subsection{Reflexion und Brechung}

Bei der Ellipsometrie ändert sich aufgrund von Reflexion an einer Grenzfläche zweier Materialien unterschiedlich optischer Dichte der Polarisationszustand des Lichtes. Im nächsten Kapitel wird detailierter auf die Grundlagen und physikalischen Gesetzmäßigkeiten der Wechselwirkung von Licht an einer Grenzfläche eingegangen.

Trifft Licht beispielsweise auf die Luft-Wasser-Grenzfläche, so wird ein Teil des Lichtes von der Wasseroberfläche reflektiert, der andere Teil ins Wasser gebrochen. Somit spaltet sich der einfallende Lichtkegel in Teilstrahlen auf (Abbildung 2.4).

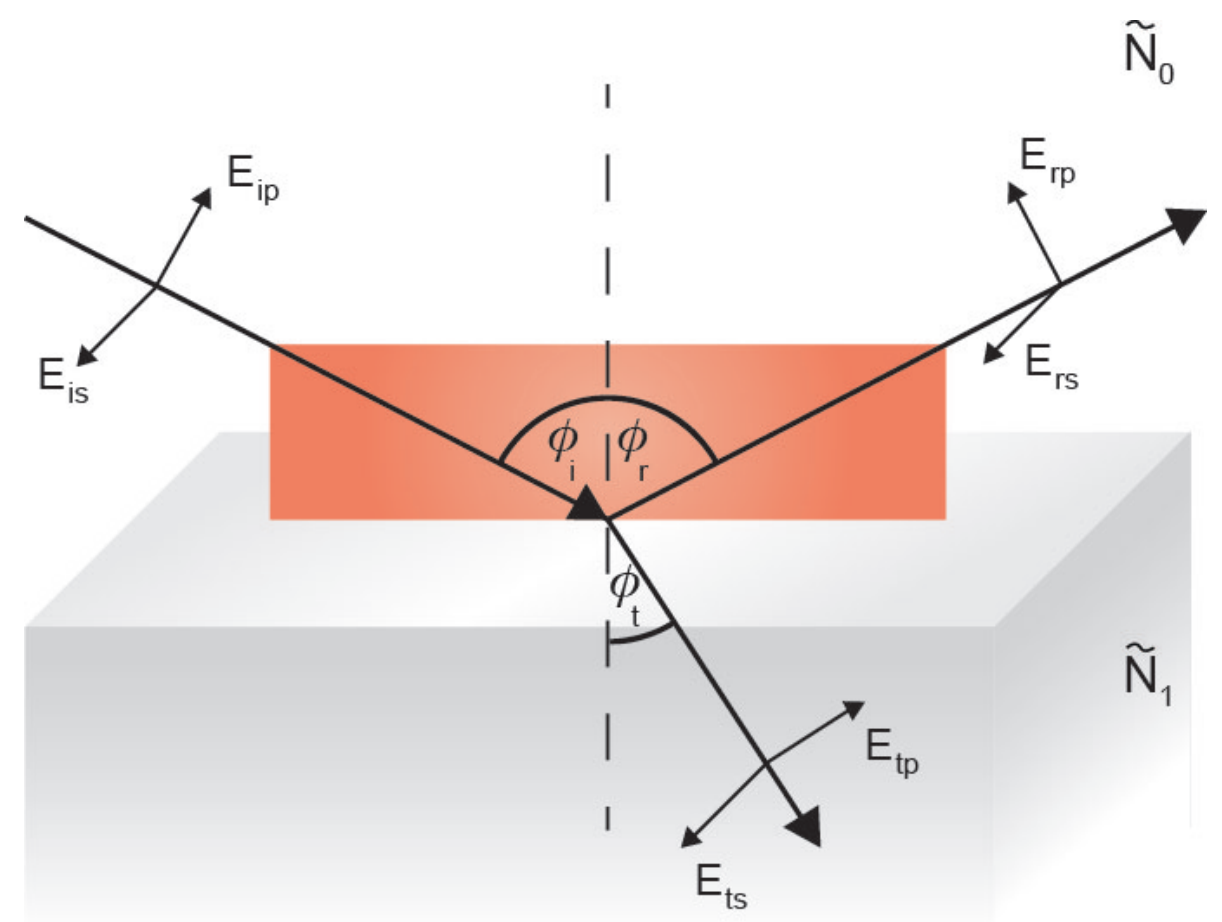

Abbildung 2.4 Darstellung des Reflexionsverhaltens von Licht an einer Grenzfläche zweier optisch verschiedener Materialien $\left(\tilde{N}_{0}<\tilde{N}_{1}\right)$.

Der einfallende und der reflektierte Lichtstrahl befinden sich in der Einfallsebene (rote Fläche). Der Winkel zwischen dem Lot, die Senkrechte zur Reflexionsfläche (gestrichelte Linie), und dem einfallenden Lichtkegel wird Einfallswinkel $\phi_{\mathrm{i}}$ bzw. auch AOI (angle of incidence) genannt. Dieser entspricht gleichzeitig dem Reflexionswinkel $\phi_{\mathrm{r}}$. In Abbildung 2.4 sind die Teilkomponenten der Wellengleichung des einfallenden, reflektierten sowie transmittierten Lichtstrahls visualisiert. Die Amplitude des elektrischen Feldes der Welle, die in der Einfallsebene oszilliert, wird mit $E_{\mathrm{p}}$ bezeichnet. Analog dazu wird die senkrecht dazu stehende Amplitude des elektrischen Feldes der zweiten Teilwelle mit $E_{\mathrm{s}}$ bezeichnet. Neben der Reflexion wird die Strahlung auch teilweise gebrochen bzw. transmittiert. Dabei ist der Brechungswinkel $\phi_{\mathrm{t}}$ abhängig vom Verhältnis der komplexen Brechungsindizes der beiden Materialien $\tilde{N}_{0}$ und $\tilde{N}_{1}$ sowie vom Einfallswinkel $\phi_{\mathrm{i}}$. Das physikalische Verhältnis der 
beiden komplexen Brechungsindizes wird durch das Snelliussche Brechungsgesetz beschrieben $[22,33]$ :

$$
\tilde{N}_{0} \sin \phi_{\mathrm{i}}=\tilde{N}_{1} \sin \phi_{\mathrm{t}}
$$

mit $[22,22]$

$$
\tilde{N}=n-i k
$$

Bei der physikalischen Beschreibung der Reflexion ist das Verhältnis der Lichtintensität des Lichtstrahls vor und nach Reflexion relevant. Das Verhältnis der Teilkomponenten s und p wird durch die Fresnelschen Reflexionskoeffizienten beschrieben $[22,72]$ :

$$
\begin{aligned}
& r_{\mathrm{p}}=\frac{E_{\mathrm{rp}}}{E_{\mathrm{ir}}}=\frac{\tilde{N}_{0} \cos \phi_{\mathrm{t}}-\tilde{N}_{1} \cos \phi_{\mathrm{i}}}{\tilde{N}_{0} \cos \phi_{\mathrm{t}}+\tilde{N}_{1} \cos \phi_{\mathrm{i}}} \\
& r_{\mathrm{s}}=\frac{E_{\mathrm{rs}}}{E_{\mathrm{is}}}=\frac{\tilde{N}_{0} \cos \phi_{\mathrm{i}}-\tilde{N}_{1} \exp \phi_{\mathrm{t}}}{\tilde{N}_{0} \exp \phi_{\mathrm{i}}+\tilde{N}_{1} \exp \phi_{\mathrm{t}}}
\end{aligned}
$$

Für die Transmission ergeben sich folgende Ausdrücke für die Fresnelschen Transmissionskoeffizienten:

$$
\begin{aligned}
& t_{\mathrm{p}}=\frac{E_{\mathrm{tp}}}{E_{\mathrm{ip}}}=\frac{2 \tilde{N}_{0} \cos \phi_{\mathrm{t}}}{\tilde{N}_{0} \cos \phi_{\mathrm{t}}+\tilde{N}_{1} \cos \phi_{\mathrm{i}}} \\
& t_{\mathrm{s}}=\frac{E_{\mathrm{ts}}}{E_{\mathrm{is}}}=\frac{2 \tilde{N}_{0} \cos \phi_{\mathrm{i}}}{\tilde{N}_{0} \exp \phi_{\mathrm{i}}+\tilde{N}_{1} \exp \phi_{\mathrm{t}}}
\end{aligned}
$$

$r$ und $t$ repräsentieren die Fresnelschen Koeffizienten, $E$ die Amplitude des elektrischen Feldes, $\tilde{N}$ den komplexen Brechungsindex und $\phi$ den Winkel. Zusätzlich stehen die Indizes p für parallel, s für senkrecht, 0 und 1 für die jeweiligen Materialien, i für einfallend, $\mathrm{r}$ für reflektiert und $t$ für transmittiert. Die Ausdrücke für die Reflexion $R_{\mathrm{s}}$ und $R_{\mathrm{p}}$ lauten definitionsgemäß [22, 39]:

$$
R_{\mathrm{p}}=\left|r_{\mathrm{p}}\right|^{2} \quad R_{\mathrm{s}}=\left|r_{\mathrm{s}}\right|^{2}
$$

Besondere Fälle:

Für $n_{0}<n_{1}, \mathrm{k}=0$ : 


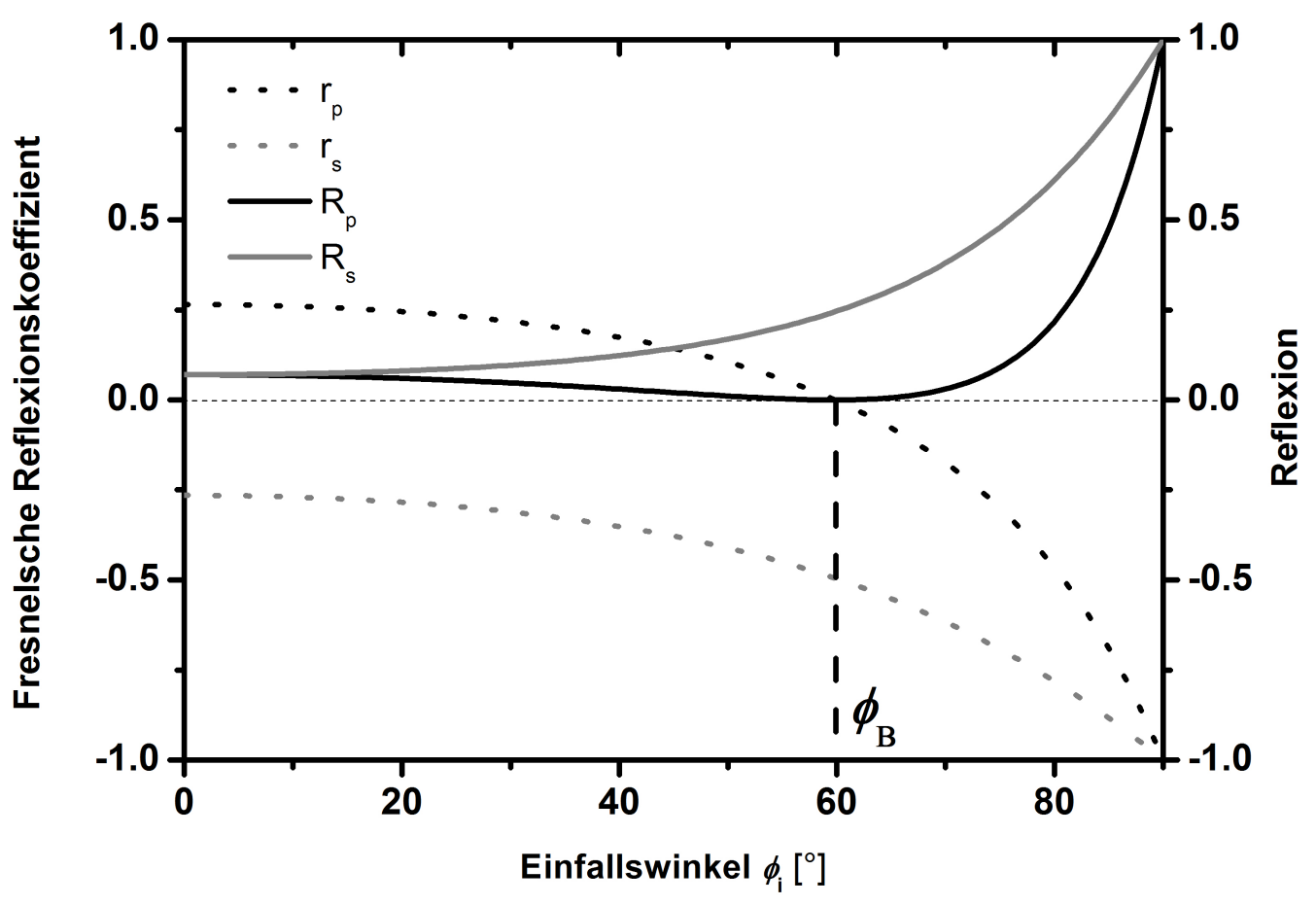

\begin{abstract}
Abbildung 2.5 Graphische Darstellung der Fresnelschen Reflexionskoeffizienten und der Reflexion in Abhängigkeit des Einfallswinkels an der Luft-Glas(SF10)-Grenzfläche bei einer Wellenlänge von $658 \mathrm{~nm}$.
\end{abstract}

In Abbildung 2.5 sind die Fresnelschen Reflexionskoeffizienten bzw. Reflexionswerte an der Luft-Glas-Grenzfläche $\left(n_{0}=1, n_{1}(\mathrm{SF} 10)=1.72\right.$, bei $\left.658 \mathrm{~nm}\right)$ in Abhängigkeit vom Einfallswinkel $\phi_{i}$ dargestellt. Der Fresnelsche Reflexionskoeffizient $r_{s}$ verläuft über den gesamten Einfallswinkelbereich negativ. Im Gegensatz dazu kann der Fresnelsche Reflexionskoeffizient $r_{\mathrm{p}}$ positive Werte bis hin zu -1 aufweisen. Dabei schneidet der Verlauf an einem Punkt die x-Achse. An diesem Punkt besitzt $R_{\mathrm{p}}$ ein Minimum. Die p-Komponente wird komplett transmittiert und die Reflexion der Welle parallel zur Einfallsebene bleibt aus. Lediglich die Komponente der Welle, die senkrecht zur Einfallsebene verläuft, wird reflektiert. Dieser Punkt bzw. Winkel wird auch als Brewsterwinkel $\phi_{\mathrm{B}}$ bezeichnet. Es gilt folgender mathematischer Zusammenhang [22, 41]:

$$
\tan \phi_{\mathrm{B}}=\frac{n_{1}}{n_{0}}
$$


Für $n_{0}<n_{1}, \mathrm{k} \neq 0$ :

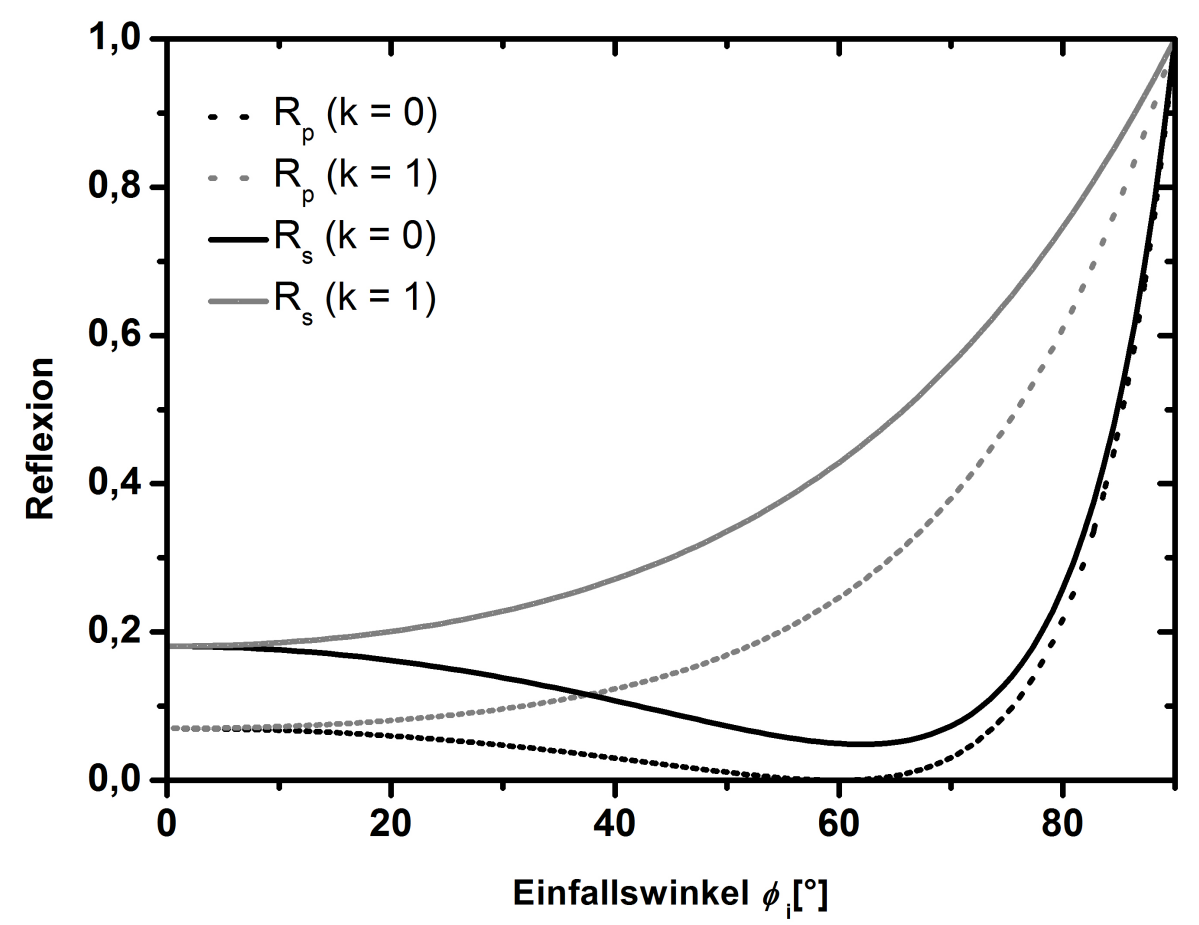

Abbildung 2.6 Reflexion in s- und p-Richtung, aufgetragen gegen den Einfallswinkel an der Grenzfläche zwischen Luft und Glas mit und ohne Absorption ( $\mathrm{n}=1.72, \mathrm{k}=0$ bzw. $\mathrm{k}=1$ ) bei einer Wellenlänge von $658 \mathrm{~nm}$.

Bei nicht transparenten Materialien ist die Reflexion $R_{\mathrm{p}}$ stets ungleich $0 . R_{\mathrm{p}}$ kann jedoch ein lokales Minimum besitzen, das als Pseudo-Brewsterwinkel (Abbildung 2.6) bezeichnet wird. 
Für $n_{0}>n_{1}$ :

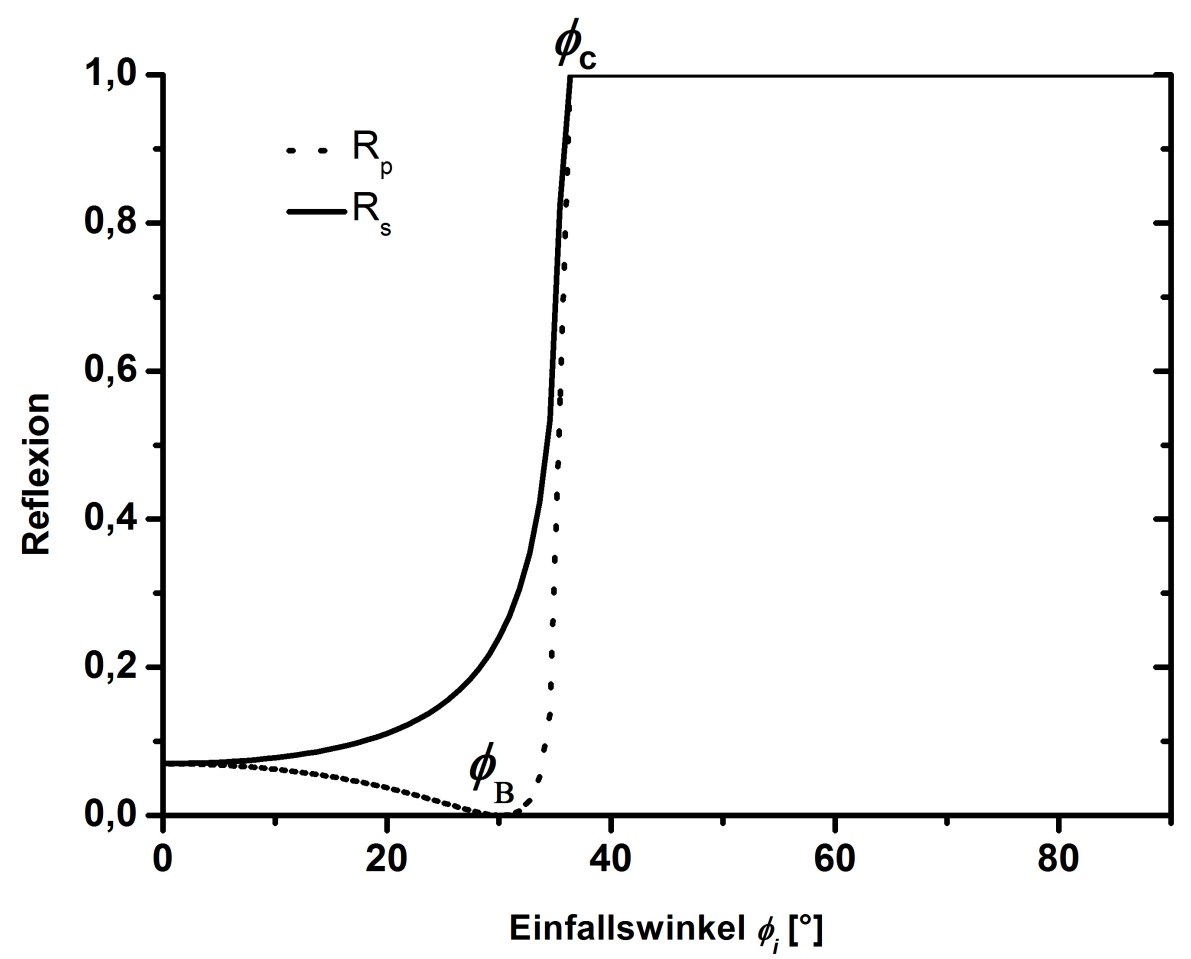

Abbildung 2.7 Reflexion für s- und p-Polarisation, aufgetragen gegen den Einfallswinkel an der Grenzfläche zwischen Glas $(\mathrm{n}=1.72)$ und Luft bei einer Wellenlänge von $658 \mathrm{~nm}$.

Ist $n_{0}$ jedoch das optisch dichtere Medium im Vergleich zu $n_{1}$, dann ist der Brechungswinkel größer als der Einfallswinkel. Aufgrund dieser Tatsache gibt es einen kritischen Winkel, bei dem der gebrochene Lichtstrahl in Totalreflexion übergeht (Abbildung 2.7). Ab dem kritischen Winkel $\phi_{\mathrm{c}}$ geht das Licht ins evaneszente Feld über [22, 42]. Handelt es sich um ein Material, das Licht absorbiert, spricht man von abgeschwächter Totalreflexion (attenuated total reflection ATR). Dieser Effekt wird unter anderem bei Messmethoden wie der SPR (surface plasmon resonance) und der ATR-FTIR-Spektroskopie (Kapitel 3.2.1) genutzt. 


\subsection{Optische Interferenz}

Dieses Unterkapitel beschreibt das Phänomen der Interferenz von Licht bei Einschichtsystemen. Zunächst wird das Reflexionsmuster bei isotropen Materialien aufgezeigt. Auf einen allgemeineren Ansatz mittels Tensorbeschreibung wird im weiteren Verlauf diesem Unterkapitels eingegangen. Die Herleitung der Gleichungen sind in "Spectroscopic ellipsometry" von H. FuJIwara [22, 44] zu finden.

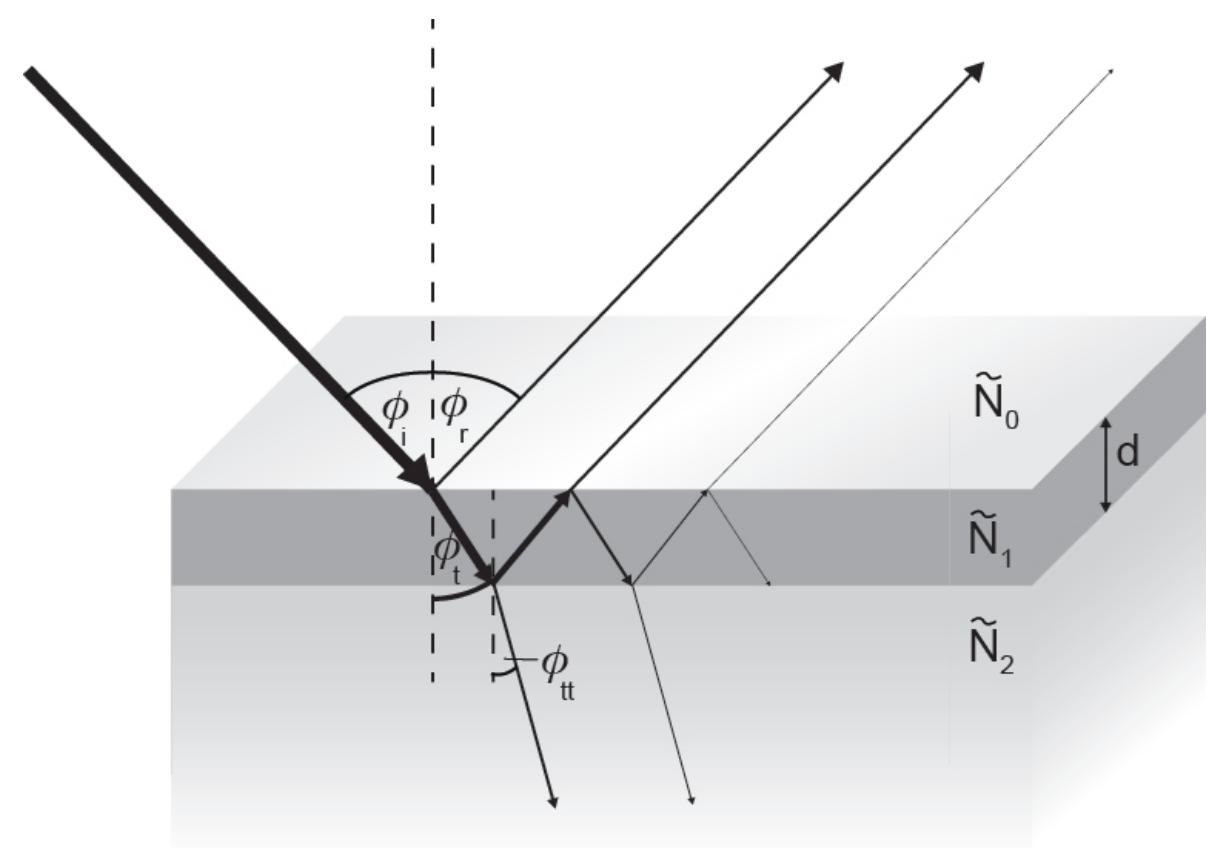

Abbildung 2.8 Interferenzmuster eines an einem Dünnschichtsystem reflektierten bzw. gebrochenen Lichtstrahls.

Abbildung 2.8 veranschaulicht das Interferenzmuster von Licht bei einem Einschichtsystem mit transparenten Materialien. Bei Dünnschichtsystemen gibt es mehr als nur eine Grenzfläche, an der das Licht reflektiert und nach Brechung durch die Schicht bzw. das Substrat transmittiert. An der ersten Grenzfläche wird der einfallende Lichtstrahl, wie im vorherigen Kapitel beschrieben, reflektiert und entsprechend der optischen Dichte des Materials gebrochen. Das an der ersten Grenzfläche gebrochene Licht wird nach Transmission erneut teilweise reflektiert und nach Brechung wiederum transmittiert. Zur Beschreibung des Reflexions- und Transmissionsverhaltens eines Einschichtsystems werden nachstehend zwei Ansätze - jener nach Fresnel und jener mittels der 4x4-Matrix-Methode - erläutert.

\section{Fresnel}

Bei der mathematischen Beschreibung durch die Fresnelschen Gleichungen des Systems gelten folgende Annahmen:

- Das Substrat mit einem komplexen Brechungsindex $\tilde{N}_{2}=n_{2}-i k_{2} \in \mathbb{C}$ zeige keinen Rückseitenreflex. [14]. 
- Alle Schichten seien homogen, isotrop und planar.

Dadurch entsteht ein Reflexionsmuster, so lauten die Fresnelschen Koeffizienten für die Reflexion bzw. Transmission wie folgt $[22,46]$ :

$$
\begin{aligned}
& r_{012 \mathrm{p}}=\frac{r_{01 \mathrm{p}}+r_{12 \mathrm{p}} \exp (-i 2 \beta)}{1+r_{01 \mathrm{p}} r_{12 \mathrm{p}} \exp (-i 2 \beta)} \quad r_{012 \mathrm{~s}}=\frac{r_{01 \mathrm{~s}}+r_{12 \mathrm{~s}} \exp (-i 2 \beta)}{1+r_{01 \mathrm{~s}} r_{12 \mathrm{~s}} \exp (-i 2 \beta)} \\
& t_{012 \mathrm{p}}=\frac{t_{01 \mathrm{p}} t_{12 \mathrm{p}} \exp (-i \beta)}{1+r_{01 \mathrm{p}} r_{12 \mathrm{p}} \exp (-i 2 \beta)} \quad t_{01 \mathrm{~s}}=\frac{t_{01 \mathrm{~s}}+t_{12 \mathrm{~s}} \exp (-i \beta)}{1+r_{01 \mathrm{~s}} r_{12 \mathrm{~s}} \exp (-i 2 \beta)} \\
& R_{\mathrm{p}}=\left|r_{012 \mathrm{p}}\right|^{2} \quad R_{\mathrm{s}}=\left|r_{012 \mathrm{~s}}\right|^{2}
\end{aligned}
$$

mit $[22,44]$

$$
\beta=\frac{2 \pi d}{\lambda} \tilde{N}_{1} \cos \phi_{\mathrm{t}}=\frac{2 \pi d}{\lambda} \sqrt{\tilde{N}_{1}^{2}-\tilde{N}_{0}^{2} \sin ^{2} \phi_{\mathrm{i}}}
$$

Hierbei steht $\beta$ für die Phasenveränderung der Welle, $d$ für die Schichtdicke des Films und $\lambda$ für die jeweilige Wellenlänge. Die Zahlen an den Fresnelschen Koeffizienten stehen für die jeweilige Grenzfläche.

Auch anisotrope Materialien können über Fresnelschen Ansatz beschrieben werden. Auf diesen Aspekt wird in dieser Arbeit indes nicht weiter eingegangen und lediglich auf die entsprechenden Kapitel im Fujiwara verwiesen [22, 222].

\section{4x4-Matrix-Methode}

Während in dem beschriebenen Formalismus nach Fresnel eine optische Anisotropie der reale Probensysteme ausgeschlossen ist, liefert die 4x4-Matrix-Methode einen allgemeineren Ansatz. Dieser findet auch in der folgenden Arbeit bei der Modellierung der optischen Parameter Anwendung [22]. Über dieser Methode werden die Reflexionskoeffizienten des Systems als Lösung der maxwellschen Gleichungen bestimmt. Dazu wird die Berreman-Gleichung wie folgt aus den Maxwellgleichungen hergeleitet [10]:

$$
\frac{\partial \Psi}{\partial z}=i \frac{\omega}{c} \Delta_{\mathrm{B}} \Psi
$$

Dabei steht $\Psi$ für einen Vektor, dessen Elemente das elektromagnetische Feld repräsentieren, während $\Delta_{\mathrm{B}}$ einer $4 \mathrm{x} 4$-Matrix von Berreman, die einen Tensor beschreibt, entspricht. Nach Anwendung mathematischer Operationen ergibt sich folgender allgemeiner Ausdruck in Form der Transfermatrix T, ${ }^{7}$

$$
\left(\begin{array}{c}
E_{\mathrm{is}} \\
E_{\mathrm{rs}} \\
E_{\mathrm{ip}} \\
E_{\mathrm{rp}}
\end{array}\right)=\mathbf{T}\left(\begin{array}{c}
E_{\mathrm{ts}} \\
0 \\
E_{\mathrm{tp}} \\
0
\end{array}\right)=\left[\begin{array}{llll}
T_{11} & T_{12} & T_{13} & T_{14} \\
T_{21} & T_{22} & T_{23} & T_{24} \\
T_{31} & T_{32} & T_{33} & T_{34} \\
T_{41} & T_{42} & T_{43} & T_{44}
\end{array}\right]\left(\begin{array}{c}
E_{\mathrm{ts}} \\
0 \\
E_{\mathrm{tp}} \\
0
\end{array}\right)
$$

\footnotetext{
${ }^{7}$ Herleitung des Ausdrucks in [22, $\left.226 \mathrm{ff}\right]$
} 
Für eine isotrope Probe lassen sich die Reflektionskoeffizienten mittels Tensoren beschreiben [22, 230]

$$
\begin{aligned}
& r_{\mathrm{pp}}=\left(\frac{E_{\mathrm{rp}}}{E_{\mathrm{ip}}}\right)_{E_{\mathrm{is}}=0}=\frac{T_{11} T_{43}-T_{13} T_{41}}{T_{11} T_{33}-T_{13} T_{31}} \\
& r_{\mathrm{ss}}=\left(\frac{E_{\mathrm{rs}}}{E_{\mathrm{is}}}\right)_{E_{\mathrm{ip}}=0}=\frac{T_{21} T_{33}-T_{23} T_{31}}{T_{11} T_{33}-T_{13} T_{31}}
\end{aligned}
$$

Der somit erhaltene Ausdruck beschreibt das Modell exakt, weil er direkt aus den Maxwellgleichungen hergeleitet worden ist und keine weiteren Annahmen getroffen wurden. Selbst für ein einfaches ideales Einschichtsystem werden die physikalischen Beschreibungen des Reflektionsverhaltens recht komplex und umfangreich.

Die allgemeine Form der Reflexion an einer Probe $\mathrm{S}$ in Form einer Jones-Matrix lautet:

$$
\mathbf{S}_{\text {anisotrop }}=\left[\begin{array}{cc}
r_{\mathrm{pp}} & r_{\mathrm{ps}} \\
r_{\mathrm{sp}} & r_{\mathrm{ss}}
\end{array}\right]
$$

Für eine Probe, deren Schichten sowohl isotrop, homogen und planar sind und bei der das System kein depolarisierendes Verhalten zeigt, vereinfacht sich die JonesMatrix bezüglich des Reflexionsverhaltens [22, 86]

$$
\mathbf{S}_{\text {isotrop }}=\left[\begin{array}{cc}
r_{\mathrm{p}} & 0 \\
0 & r_{\mathrm{s}}
\end{array}\right]
$$

und es ergeben sich die Fresnelschen Reflexionskoeffizienten.

Bei mehr als nur einer Schicht auf dem Substrat werden die physikalischen Beschreibungen noch komplexer, weshalb für Mehrschichtsysteme auf das Kapitel im Fujiwara verwiesen sei. 


\subsection{Fundamentalgleichung}

Auf welche Weise sind die optischen Parameter der Materialien und die Schichtdicke bei Einschichtsystemen mit den ellipsometrischen Parametern verknüpft? Das Zusammenspiel von Reflexion und Polarisation wird in der Fundamentalgleichung der Ellipsometrie wie folgt zusammengefasst $[22,82]$ :

$$
\tan \Psi \exp (i \Delta)=\frac{R_{\mathrm{p}}}{R_{\mathrm{s}}}=\rho\left(\tilde{N}_{0}, \tilde{N}_{1}, \tilde{N}_{2}, d, \phi_{\mathrm{i}}, \lambda\right)
$$

Über diese Gleichung werden die beiden Variablen $\Delta$ und $\Psi$, die bei der Ellipsometrie als Messgrößen bestimmt werden, über die Reflexionskoeffizienten $R_{\mathrm{p}}$ und $R_{\mathrm{s}}$ mit den optischen Konstanten und der Schichtdicke verknüpft. Daraus ergibt sich eine Abhängigkeit des ellipsometrischen Wertepaars Delta und Psi vom komplexen Brechungsindex der Medien (Umgebung, Film, Substrat), von der Schichtdicke, dem Einfallswinkel und der Wellenlänge. Der Einfallswinkel und die Wellenlänge sind bekannte bzw. beim Ellipsometer vom Benutzer eingestellte Größen. Die restlichen Parameter des Probensystems werden durch geeignete Modellierung (Kapitel 3.1.3.4) unter Einbeziehung der gemessenen ellipsometrischen Parameter indirekt bestimmt. Dabei enthält $\Delta$ die Phaseninformation von $\rho$ und gibt die Änderung der Phasendifferenz (siehe Kapitel 2.2) zwischen s- und p-Welle nach und vor Reflexion des Lichtes an der untersuchten Probe an [22, 83]:

$$
\Delta=\Delta_{\mathrm{r}}-\Delta_{\mathrm{i}}
$$

mit

$$
\begin{aligned}
& \Delta_{\mathrm{r}}=\delta_{\mathrm{rp}}-\delta_{\mathrm{rs}} \\
& \Delta_{\mathrm{i}}=\delta_{\mathrm{ip}}-\delta_{\mathrm{is}}
\end{aligned}
$$

Das Verhältnis der Beträge der Reflexionskoeffizienten wird durch $\tan \Psi$ repräsentiert und steht als reelle Zahl für das Maß für den Betrag von $\rho$ :

$$
\tan \Psi=\frac{\left|R_{\mathrm{r}}\right|}{\left|R_{\mathrm{S}}\right|}
$$




\subsection{Optische Konstanten}

Diese Arbeit fokussiert sich auf amorphe Materialien und die Untersuchung isotroper bzw. uniaxialer anisotroper Schichten. Neben der Beschreibung von optisch isotropen Materialien wird die Thematik der optischen Konstanten kurz für anisotrope Materialien aufgeführt. Für einen tieferen Einblick hinsichtlich anisotroper Materialien sei auf die einschlägige Literatur „Spectroscopic ellipsometry“ von $\mathrm{H}$. FujIWARA [22] sowie „Handbook of ellipsometry" von H. Tompkins und E. A. Irene [55] verwiesen.

\subsubsection{Isotropes Medium}

Wie anhand der Formeln in den vorherigen Kapiteln ersichtlich ist, bestimmt der komplexe Brechungsindex das Reflexions- und Polarisationsverhalten von Licht. Der komplexe Brechungsindex für ein isotropes $\left(\tilde{N}_{\mathrm{x}}=\tilde{N}_{\mathrm{y}}=\tilde{N}_{\mathrm{z}}{ }^{8}\right)$ Material lautet [55, 29]:

$$
\tilde{N}(\lambda)=n(\lambda)-i k(\lambda)
$$

Der komplexe Brechungsindex ist die Differenz aus dem realen Brechungsindex $n$, dem Produkt der komplexen Zahl $i$ und dem Extinktionskoeffizienten $k$.

Der Extinktionskoeffizient ist - wie nachstehend formuliert - direkt mit dem Absorptionskoeffizienten $\alpha$ des Materials verknüpft [22, 23]:

$$
\alpha(\lambda)=\frac{4 \pi k(\lambda)}{\lambda}
$$

Außerdem gilt $[22,29]$ :

$$
\tilde{N}^{2}=\varepsilon
$$

Die optischen Konstanten werden zumeist in der Literatur als komplexe dielektrische Funktion $\varepsilon$ oder komplexe Leitfähigkeit $\sigma$ angegeben. Die mathematischen Beziehungen zwischen den unterschiedlichen Formen der optischen Konstanten ist in Tabelle 2.2 zusammengefasst:

\footnotetext{
${ }^{8}$ Gilt für kubische Kristallstrukturen oder amorphe Materialien [55, 29]
} 


\begin{tabular}{|c|c|c|}
\hline & Realteil & Imaginärteil \\
\hline $\begin{array}{l}\text { Leitfähigkeit } \\
\sigma=\sigma_{1}-i \sigma_{2}\end{array}$ & $\sigma_{1}=\omega \varepsilon_{0} \varepsilon_{2}$ & $\overline{\sigma_{2}}=-\omega \varepsilon_{0}\left(\varepsilon_{1}-1\right)$ \\
\hline $\begin{array}{c}\text { Dielektrische } \\
\text { Funktion } \\
\varepsilon=\varepsilon_{1}-i \varepsilon_{2} \\
\end{array}$ & $\begin{array}{c}\varepsilon_{1}=1-\sigma_{2} /\left(\omega \varepsilon_{0}\right) \\
\varepsilon_{1}=n^{2}-k^{2}\end{array}$ & $\begin{array}{c}\varepsilon_{2}=\sigma_{1} /\left(\omega \varepsilon_{0}\right) \\
\quad \varepsilon_{2}=2 n k\end{array}$ \\
\hline $\begin{array}{l}\text { Brechungsindex } \\
\quad \tilde{N}=n-i k\end{array}$ & $\begin{array}{c}n=\sqrt{\left(\varepsilon_{1}+\sqrt{\varepsilon_{1}^{2}+\varepsilon_{2}^{2}}\right) / 2} \\
n=\varepsilon_{2} /(2 k)\end{array}$ & $\begin{array}{c}k=\sqrt{\left(-\varepsilon_{1}+\sqrt{\varepsilon_{1}^{2}+\varepsilon_{2}^{2}}\right) / 2} \\
k=\varepsilon_{2} /(2 n)\end{array}$ \\
\hline
\end{tabular}

Tabelle 2.2 Umrechnung der optischen Konstanten [55, 24].

Dabei sind der Real- und Imaginärteil der jeweiligen Funktion abhängig voneinander. So wirkt sich eine Änderung von $\varepsilon_{1}$ direkt auf $\varepsilon_{2}$ aus. Die mathematischen Abhängigkeiten werden durch die Kramers-Kronig-Beziehungen beschrieben [22, 176]:

$$
\begin{aligned}
& \varepsilon_{1}(\omega)=1+\frac{2}{\pi} P \int_{0}^{\infty} \frac{\omega^{\prime} \varepsilon_{2}\left(\omega^{\prime}\right)}{\omega^{\prime 2}-\omega^{2}} d \omega^{\prime} \\
& \varepsilon_{2}(\omega)=-\frac{2 \omega}{\pi} P \int_{0}^{\infty} \frac{\varepsilon_{1}\left(\omega^{\prime}\right)-1}{\omega^{\prime 2}-\omega^{2}} d \omega^{\prime}
\end{aligned}
$$

wobei $P$ in den Gleichung 2.33 und 2.34 für den Hauptwert folgenden Integrals steht:

$$
P \int_{0}^{\infty} d \omega^{\prime} \equiv \lim _{\delta \rightarrow 0}\left(\int_{0}^{\omega-\delta} d \omega^{\prime}+\int_{\omega+\delta}^{\infty} d \omega^{\prime}\right)
$$

\subsubsection{Anisotropes Medium}

Im Gegensatz zu isotropen Materialien, bei denen die Orientierung des Systems bei der Reflexion und Transmission des Lichtes keine Rolle spielt, besitzen anisotrope Medien eine oder zwei optische Achsen. Nach der Anzahl der optischen Achsen wird zwischen uniaxialer (mit $\tilde{N}_{\mathrm{x}}=\tilde{N}_{\mathrm{y}} \neq \tilde{N}_{\mathrm{z}}{ }^{9}$ ) und biaxialer $\left(\operatorname{mit} \tilde{N}_{\mathrm{x}} \neq \tilde{N}_{\mathrm{y}} \neq \tilde{N}_{\mathrm{z}}\right.$ $\left.{ }^{10}\right)$ Anisotropie differenziert. Häufig werden die optischen Parameter in Form eines Ellipsoids, dielektrischen Tensors oder als Jones-Matrix dargestellt.

Uniaxial anisotrope Schichten mit der optischen Achse senkrecht zur Probefläche lassen sich mittels Ellipsometrie charakterisieren. Weist das Schichtsystem jedoch in allen drei Raumrichtungen einen Unterschied in den optischen Parametern auf oder

\footnotetext{
${ }^{9}$ Gilt für rhombohedrale, tetragonale und hexagonale Kristallstrukturen [55, 29]

${ }^{10}$ Gilt für trikline, monokline und orthorhombische Kristallstrukturen [55, 29]
} 
sind die optischen Achsen eines uniaxialen System nicht senkrecht zur Probenoberfläche kommt es zu einer Kreuzpolarisation bei der Reflektion des elliptischen Lichtes ${ }^{11}[22$, 209]. Durch geeignete Ausrichtung der Proben zeigt das anisotrope Probensystem ein pseudo-isotropes Verhalten. Dazu werden die optischen Achsen des Schichtsystem durch Rotation so ausgerichtet, dass sie sich senkrecht oder parallel zur Einfallsebene des Lichtes befindet [22, 221]. Als alternative Messmethode sei auf die Müller-Matrix-Ellipsometrie verwiesen [22, 111].

\subsubsection{Dispersion}

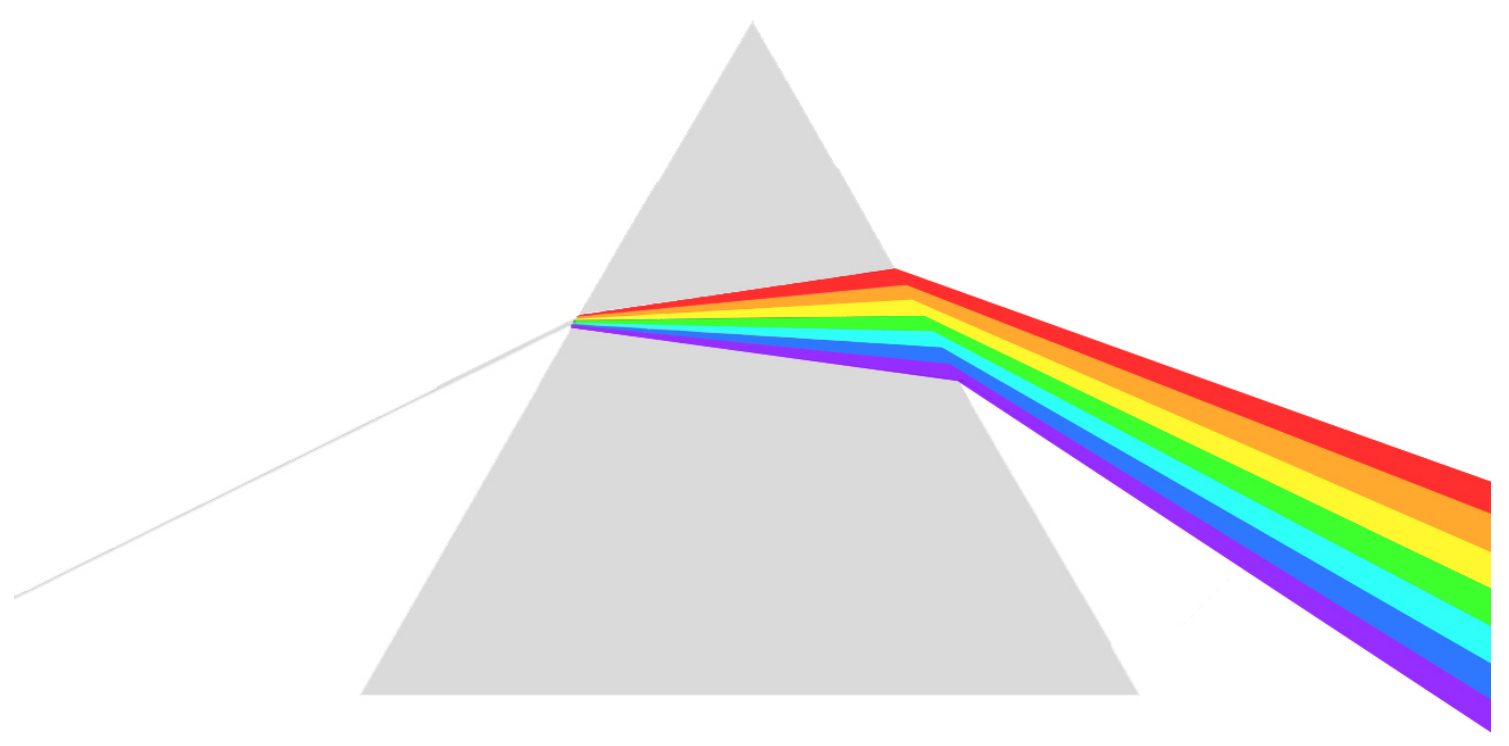

Abbildung 2.9 Dispersion von Weißlicht an einem Glasprisma.

Wie in Gleichung 2.30 ersichtlich, sind die optischen Parameter eines Mediums abhängig von der Wellenlänge. Ein reales Beispiel, das den physikalischen Effekt visualisiert, ist in Abbildung 2.9 gezeigt. Aufgrund der Wellenlängenabhängigkeit des Brechungsindex' kommt es bei der Brechung und anschließender Transmission von Weißlicht an einem Glasprisma zur Aufspaltung in die einzelnen Farben.

In Abbildung 2.10 sind der Real- $\left(\varepsilon_{1}\right.$, schwarz) und Imaginärteil $\left(\varepsilon_{2}\right.$, blau $)$ der dielektrischen Konstanten in Abhängigkeit von der Wellenzahl $\tilde{\nu}$ vom UV- bis hin zum IR-Bereich logarithmisch aufgetragen. Die durchgezogene Linie zeigt die Dispersion eines Materials, das sowohl atomare als auch elektrische Polarisation besitzt. Die gestrichelte Linie entspricht einem Medium mit freien Elektronen bzw. metallischem Charakter.

${ }^{11} r_{p s} \neq 0, r_{s p} \neq 0$ vgl. Gleichung 2.23 


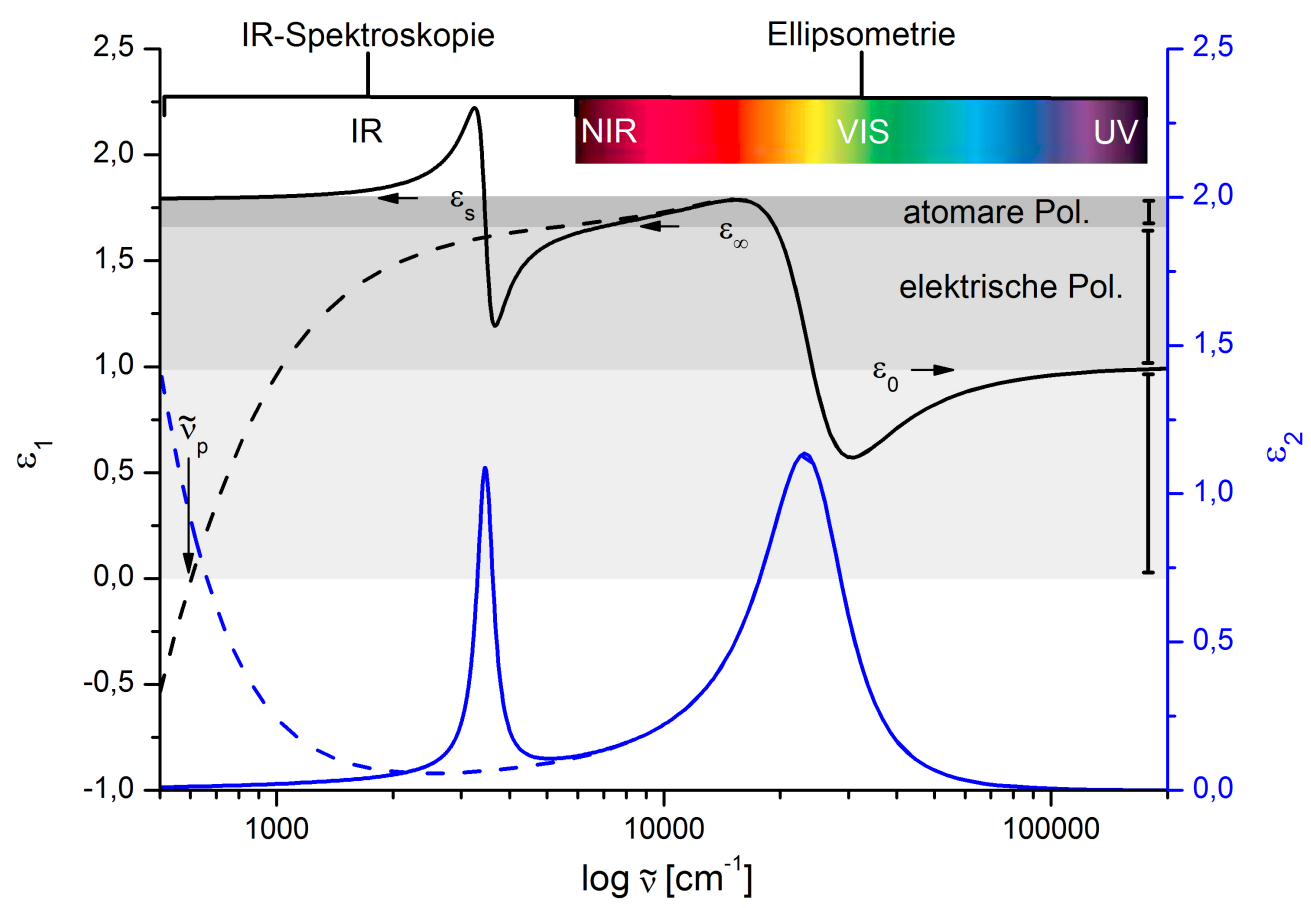

Abbildung 2.10 Verlauf der dielektrischen Konstanten zweier Materialien in Abhängigkeit von der Wellenzahl.[22, 29]

Zunächst betrachten wir nur Material mit sowohl atomarer als auch elektrische Polarisation. Im IR-Bereich $\left(\tilde{\nu} \leq 5880 \mathrm{~cm}^{-1}\right)$ entspricht der Realteil $\varepsilon_{1}$ der statischen Dielektrizitätskonstante $\varepsilon_{\mathrm{s}}$, die sich aus den Beiträgen atomarer und elektrischer Polarisation zusammensetzt. ${ }^{12}$ Beide Polarisationen werden im klassischen Modell (Lorentz-Oszillator, siehe Kapitel 2.6.4.1) als schwingende Feder betrachtet. Trifft nun Licht mit der entsprechenden Wellenzahl auf das Medium, führt zur Resonanz. Das Licht wird absorbiert und sorgt für Schwingung. Die Abbildung 2.10 zeigt für Material 1 zwei Absorptionspeaks im Imaginärteil $\varepsilon_{2}$. Allgemein liegen die Resonanzschwingungen für atomare Polarisation im IR-Bereich. Resonanzen für elektrische Polarisation tauchen im energiereicheren Bereich (UV/VIS Bereich, $\tilde{\nu}=50000-16666 \mathrm{~cm}^{-1}$ ) auf. Im Wellenzahlbereich, der nur elektrische Polarisation im Medium aufweist, wird der Realteil $\varepsilon_{1}$ zur hochfrequenten dielektrischen Konstante $\varepsilon_{\infty}$. Mit zunehmender Wellenzahl steigt der Energiegehalt des Lichtes und die Absorption des Lichtes in Form von elektrischer Polarisation bleibt aus. Der Realteil $\varepsilon_{1}$ nähert sich dem Wert für die Dielektrizitätskonstante im Vakuum $\varepsilon_{0}=1$. Folglich hängen die komplexen dielektrischen Konstanten des Materials von den jeweiligen Resonanzfrequenzen der dielektrischen Polarisation ab. Im Gegensatz dazu besitzt das andere Material freie Elektronen und das Licht wird im IR-Bereich absorbiert. Die Absorption aufgrund freier Elektronen nimmt bei kleineren Wellenzahlen zu. Der Punkt, nach dem der Realteil $\varepsilon_{1}$ negative Werte besitzt, wird als

\footnotetext{
${ }^{12}$ In Kristallen ohne Ionencharakter (z.B Si) ist $\varepsilon_{\mathrm{s}}=\varepsilon_{\infty}$, da keine atomare Polarisation auftritt.
} 
Plasmawellenzahl $\tilde{\nu}_{\mathrm{p}}$ bezeichnet. Im oberen Teil der Abbildung sind die Bereiche für die spektroskopische Ellipsometrie und IR-Spektroskopie eingezeichnet. Aufgrund der Wellenlänge bzw. des Energiegehalts des Lichts wird bei der Ellipsometrie nur elektrische Polarisation beobachtet bzw. detektiert.

\subsubsection{Dielektrische Funktionsmodelle}

Dieses Unterkapitel beschäftigt sich mit der physikalischen Beschreibung der wichtigsten Dispersionsmodelle. Alle hier beschriebenen Modelle lassen sich aus dem physikalischen Modell des Lorentz-Oszillators ableiten. Die dielektrischen Konstanten des Lorentz-Oszillators werden in verkürzter Form hergeleitet. Für eine ausführlichere Beschreibung des Modells und der Herleitung der Lorentz-Funktion sei auf die einschlägige Literatur "Spectroscopic ellipsometry" von H. FUJIWARA [22, 160] und „Handbook of ellipsometry" von H. Tompkins und E. A. Irene [55, 125] verwiesen.

\subsubsection{Lorentz}

Beim Lorentz-Modell wird die Bindung zwischen dem negativ geladenen Elektron an dem positiv geladenen Kern in Form einer mechanischen Feder beschrieben. Das Elektron schwingt im viskosen Medium in x-Richtung und der Kern bleibt - aufgrund seiner größeren Masse - im Gegensatz zum Elektron. Mit dem zweiten newtonschen Gesetz erhält man folgende Beziehung:

$$
m_{\mathrm{e}} \frac{d^{2} x}{d t^{2}}=-m_{\mathrm{e}} \Gamma \frac{d x}{d t}-m_{\mathrm{e}} \omega_{0}^{2} x-e E_{0} \exp (i \omega t)
$$

$m_{\mathrm{e}}$ ist die Masse und $e$ die Ladung des Elektrons. Der erste der drei Terme beschreibt die Trägheit, die bei kleinen Geschwindigkeiten linear verläuft. Sie wird durch die Dämpfungskonstante $\Gamma$ repräsentiert. Der mittlere Term entspricht dem Hookschen Gesetz, welches das elastische Verhalten einer Feder beschreibt. Die Variable $\omega_{0}$ entspricht der Resonanzfrequenz der Feder. Der letzte Term auf der rechten Seite steht für die elektrostatische Wechselwirkung [22, 161].

Durch mathematisches Umformen erhält man die dielektrischen Konstanten [22, 161]:

$$
\begin{aligned}
& \varepsilon_{1}(E)=1+\frac{A\left(E_{0}^{2}-E^{2}\right)}{\left(E_{0}^{2}-E^{2}\right)^{2}+\Gamma^{2} E^{2}} \\
& \varepsilon_{2}(E)=\frac{A \Gamma E}{\left(E_{0}^{2}-E^{2}\right)^{2}+\Gamma^{2} E^{2}}
\end{aligned}
$$

$E_{0}$ steht für die Resonanzenergie, $\Gamma$ für die Dämpfungskonstante und $A$ für die Amplitude der Absorption. Der Wert 1 in der oberen Formel repräsentiert die Dielektrizitätskonstante des Vakuums $\varepsilon_{0}$. 


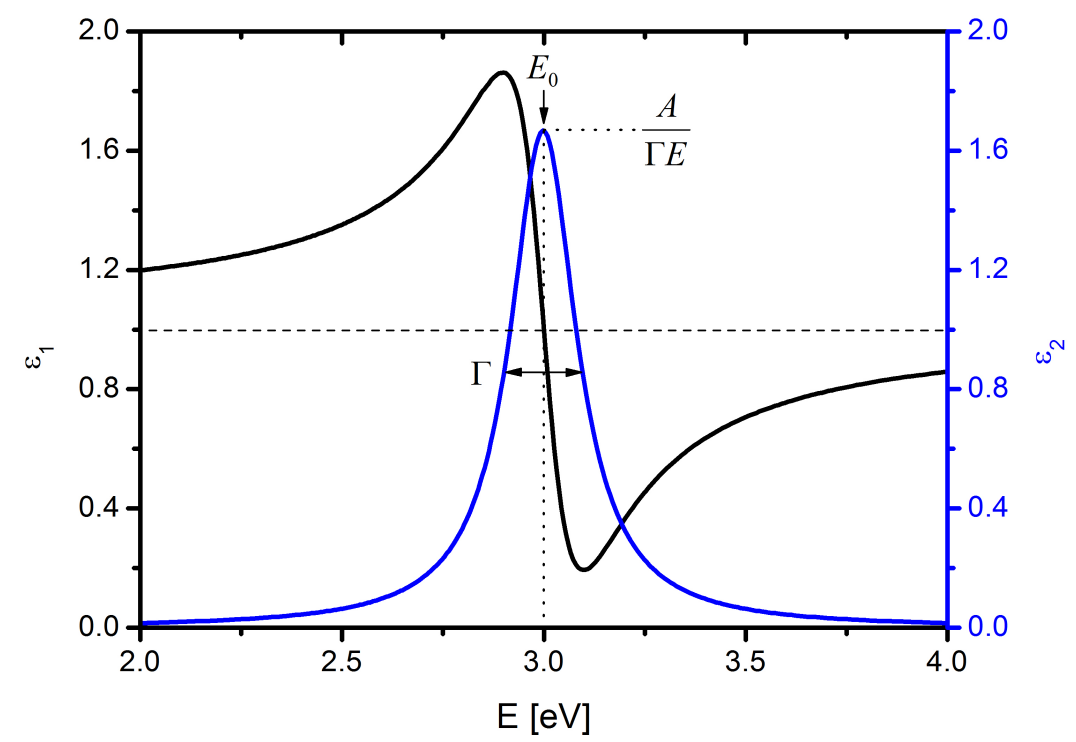

Abbildung 2.11 Dielektrische Konstanten eines LorentzOszillator mit $E_{0}=3 \mathrm{eV}, \Gamma=0.2 \mathrm{eV}$ und $A=1.66$.

Der Verlauf der dielektrischen Konstanten eines Lorentz-Oszillators ist in Abbildung 2.11 dargestellt. Bei der Resonanzenergie $E_{0}$ des Oszillators besitzt $\varepsilon_{2}$ ein Maximum, dessen Halbwertsbreite der Dämpfungskonstante $\Gamma$ entspricht. Die Höhe des Peaks repräsentiert die Amplitude $A^{13}$.

In Abbildung 2.12 werden die optischen Konstanten eines Lorentz-Oszillators $\left(E_{0}=\right.$ $\left.3 \mathrm{eV}, A=20 \mathrm{eV}^{2}, \Gamma=1 \mathrm{eV}\right)$ im Energiebereich von $1 \mathrm{eV}-5 \mathrm{eV}$ gezeigt, bei dem jeweils eine Variable unterschiedliche Werte besitzt.

${ }^{13}$ Dabei entspricht $A=\frac{e^{2} N_{\mathrm{e}}}{\varepsilon_{0} m_{\mathrm{e}} \hbar^{2}}$ 

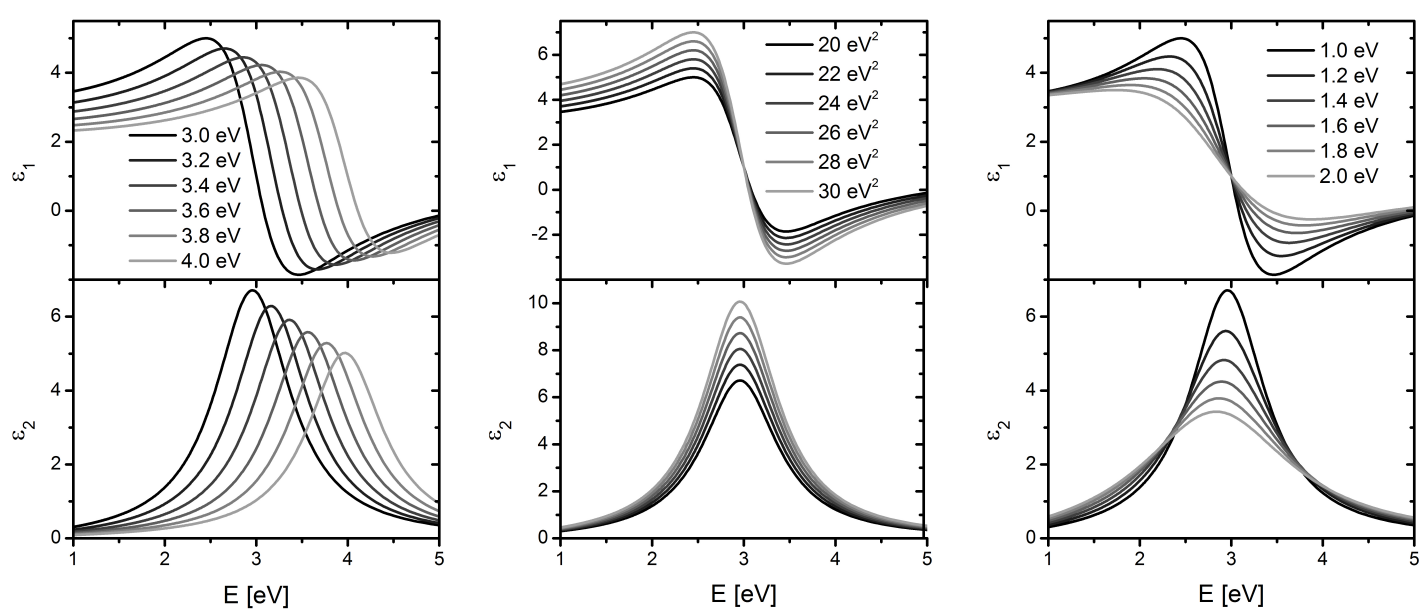

Abbildung 2.12 Graphische Darstellung der dielektrischen Konstanten des Lorentz-Oszillator in Abhängigkeit von der Energie bei variierenden Lorentz-Parametern (links $E_{0}$, mitte $A$ und rechts $\Gamma$ ).

Eine Erhöhung der Resonanzenergie führt zu einer Verschiebung des Peaks im Imaginärteil sowie des Wendepunkts im Realteil der Dispersion (Abbildung 2.12 links). Zusätzlich nimmt die Höhe des Maximums in $\varepsilon_{2}$ ab. Eine Variation der Amplitude (Abbildung 2.12 mitte) führt zu einer Erhöhung des Maximums in $\varepsilon_{2}$ und die Auslenkung in $\varepsilon_{1}$ wird verstärkt. Bei größeren Werten für der Dämpfungskonstante $\Gamma$ kommt es zu einer Verbreiterung der Lorentz-Funktion und der Maximalwert für $\varepsilon_{2}$ verringert $\operatorname{sich}^{14}$.
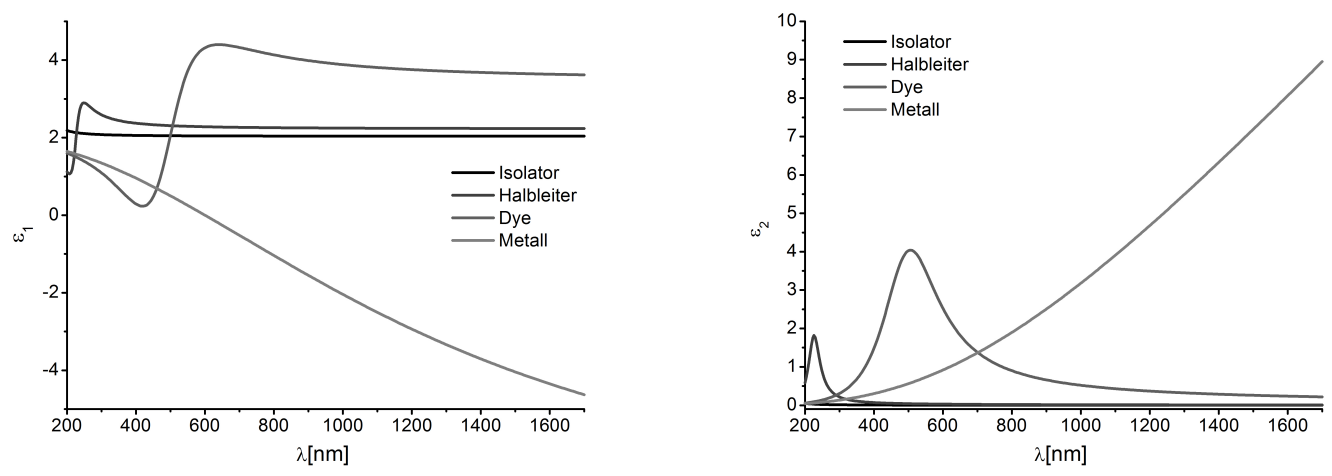

Abbildung 2.13 Vergleich der Dispersion von Isolator, Halbleiter, Dye und Metall.

Je nachdem, wo sich die jeweilige Resonanzenergie im Spektrum befindet, teilt man die Materialien in Klassen (analog zur Leitfähigkeit) ein.

${ }^{14} \propto \frac{1}{\Gamma}$ 
In Abbildung 2.13 werden die dielektrischen Parameter der vier Materialienklassen bei gleicher Dämpfungskonstante $(\Gamma=1 \mathrm{eV})$ und gleicher Stärke $\left(A=10 \mathrm{eV}^{2}\right)$ mit unterschiedlicher Resonanzenergie $E_{0}$ gezeigt. Findet ein Elektronenübergang vom Valenzband ins Leitungsband im hohen UV-Bereich statt $(\lambda<250 \mathrm{~nm})$, spricht man von einem Isolator. Dabei handelt es sich um transparente $(k=0)$ Materialien, wie beispielsweise $\mathrm{SiO}_{2}$ mit einer Resonanzenergie $E_{0}>6 \mathrm{eV}$. Die Dispersion wird häufig in der Literatur mittels Cauchy bzw. durch die Sellmeier-Funktion (Kapitel 2.6.4.2) beschrieben.

Bei Halbleitern befindet sich das Maximum von $\varepsilon_{2}$ im UV-Bereich $\left(6 \mathrm{eV}<E_{0}<\right.$ $3.5 \mathrm{eV})$. Ein typischer Vertreter ist Silizium, das im UV/VIS-Bereich absorbiert, im IR-Bereich jedoch transparent ist. Die optischen Konstanten können mittels LorentzOszillator oder dessen modifizierter Form wie Tauc-Lorentz, Tauc-Lorentz-Urbach (Kapitel 2.6.4.6) beschrieben werden.

Befindet sich ein elektronischer Übergang direkt im UV/VIS-Bereich $\left(E_{0}=3.5-\right.$ $1 \mathrm{eV}$ ), handelt es sich um ein farbiges Material (in der Literatur auch als Dye bezeichnet). Häufig zeigen Materialien mit delokalisierten $\pi$-Elektronensystem solche Absoprtionsbanden. Die optischen Parameter lassen sich durch Lorentz- oder GaussOszillatoren (Kapitel 2.6.4.1 bzw. 2.6.4.4) beschreiben.

Leitende Materialien haben eine Resonanz im IR-Bereich $\left(E_{0}<0.5 \mathrm{eV}\right)$. Typischerweise nimmt ab einer bestimmten Stelle $\varepsilon_{1}$ negative Werte an und $\varepsilon_{2}$ steigt stetig (siehe auch Abbildung 2.10). Ein solches dielektrisches Verhalten kann durch das Lorentz- oder Drude-Modell (Kapitel 2.6.4.5) parametrisiert werden.

\subsubsection{Sellmeier und Cauchy}

Beim Sellmeier-Modell betrachtet man den Wellenlängenbereich, bei dem die Dämpfungskonstante $\Gamma \rightarrow 0$ läuft und $\varepsilon_{2} \sim 0$ gilt. Dann vereinfacht sich der Realteil der Lorentz-Funktion $^{15}$ (Gleichung 2.37) zu [22, 170]:

$$
\varepsilon=\varepsilon_{1}=1+\frac{e^{2} N_{\mathrm{e}}}{\varepsilon_{0} m_{\mathrm{e}}(2 \pi c)^{2}} \frac{\lambda_{0}^{2} \lambda^{2}}{\lambda^{2}-\lambda_{0}^{2}}
$$

Diese Formel wird nach Sellmeier wie folgt beschrieben:

$$
\varepsilon_{1}=n^{2}=1+\sum_{j} \frac{B_{\mathrm{j}} \lambda^{2}}{\lambda^{2}-C_{\mathrm{j}}}
$$

In der Praxis wird häufig mehr als nur ein Sellmeier-Term genutzt, um die Dispersion zu beschreiben. Der einheitslose Sellmeier-Koeffizient B repräsentiert dabei den analytischen Parameter der Funktion. C entspricht der Resonanzwellenlänge, die sich außerhalb des Messbereichs ${ }^{16}$ befinden muss.

\footnotetext{
${ }^{15}$ in Abhängigkeit der Wellenlänge

${ }^{16}$ Kann sowohl im UV- oder IR-Bereich liegen und entspricht einer Polstelle $\left(\varepsilon_{1}(E)=\frac{A}{E_{0}^{2}-E^{2}}\right)$ in der Funktion.
} 

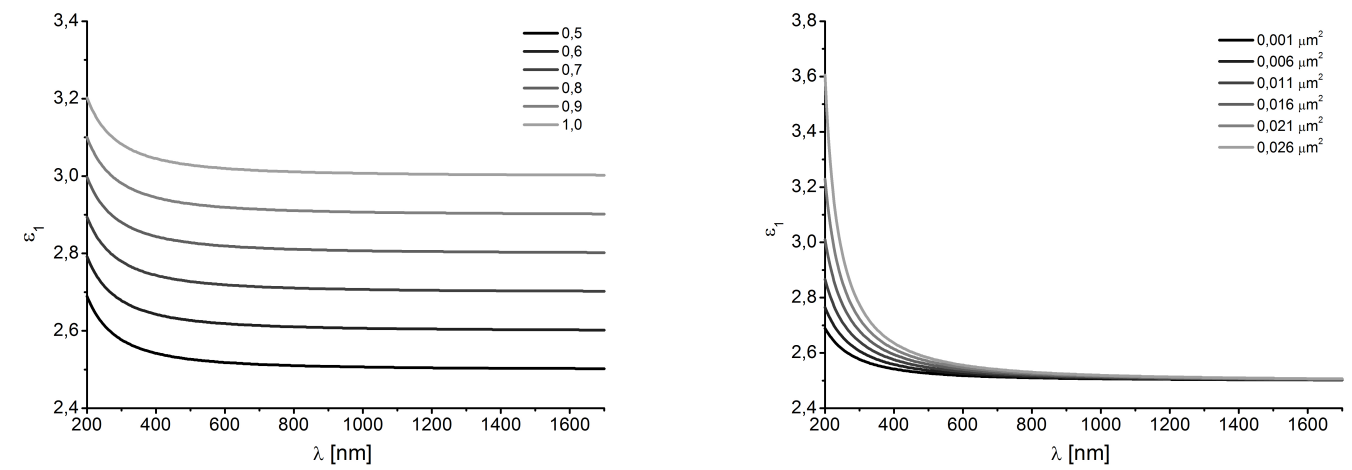

Abbildung 2.14 Sellmeier-Dispersion mit Variation der Koeffizienten $\mathrm{B}$ und $\mathrm{C}$

In Abbildung 2.14 wird die Sellmeier-Funktion mit einem Sellmeier-Term in Abhängigkeit der Koeffizienten B (links) und C (rechts) dargestellt. Die Dispersion nach Sellmeier lässt sich jedoch beliebig um weitere Terme erweitern, um die Modellierung $\mathrm{zu}$ verbessern.

Ein zweites optisches Modell für transparente Materialien wird durch die CauchyFunktion beschrieben:

$$
n=A+\frac{B}{\lambda^{2}}+\frac{C}{\lambda^{4}} \quad k=0
$$

Wie anhand der Gleichung ersichtlich ist, handelt es sich um eine Taylorreihenentwicklung. Ebenso wie die Sellmeier-Funktion ist die Cauchy-Funktion, trotz Ableitung aus dem physikalischen Modell des Lorentz-Oszillators, eine empirische Beschreibung des optischen Verhaltens transparenter Materialien. Beide Funktionen erfüllen nicht die Bedingungen von Kramers-Kronig (Gleichung $2.33+2.34$ ). Abbildung 2.15 zeigt den Brechungsindex eines Isolators, dessen optisches Verhalten durch eine Cauchy-Funktion parametrisiert wurde. 
In der oberen Abbildung wurde der Koeffizient $A$ in Schritten zu je 0.01 variiert, während die beiden anderen $\mathrm{Pa}$ rameter konstant sind $\left(B_{0}=\right.$ $3000 \mathrm{~nm}, C_{0}=1 \cdot 10^{7} \mathrm{~nm}^{2}$ ), was $\mathrm{zu}$ einer kompletten Verschiebung der Dispersion führt. In der mittleren Darstellung wird die Dispersion bei variierenden B-Werten visualisiert. Die Funktion nähert sich im NIRBereich stets dem Wert von $A$ an. Lediglich die Krümmung im UV/VIS Bereich nimmt mit steigendem B-Wert zu. In der unteren Abbildung sieht man nur den UV/VIS-Bereich der Dispersion, weil eine Variation des C-Parameters erst im UVBereich zu einer sichtbaren Veränderung des Brechungsindex' führt.

Sowohl das Cauchy- als auch das Sellmeier-Modell findet häufig bei der optischen Beschreibung von transparenten Materialien Verwendung. Ein Vergleich der beiden Modelle zeigt, dass beide Funktionen in einem Wellenlängenbereich von $\lambda=$ $400-800 \mathrm{~nm}$ nahezu deckungsgleich sind. Außerhalb dieses Bereichs der Wellenlänge können sie deutliche Abweichungen voneinander zeigen.
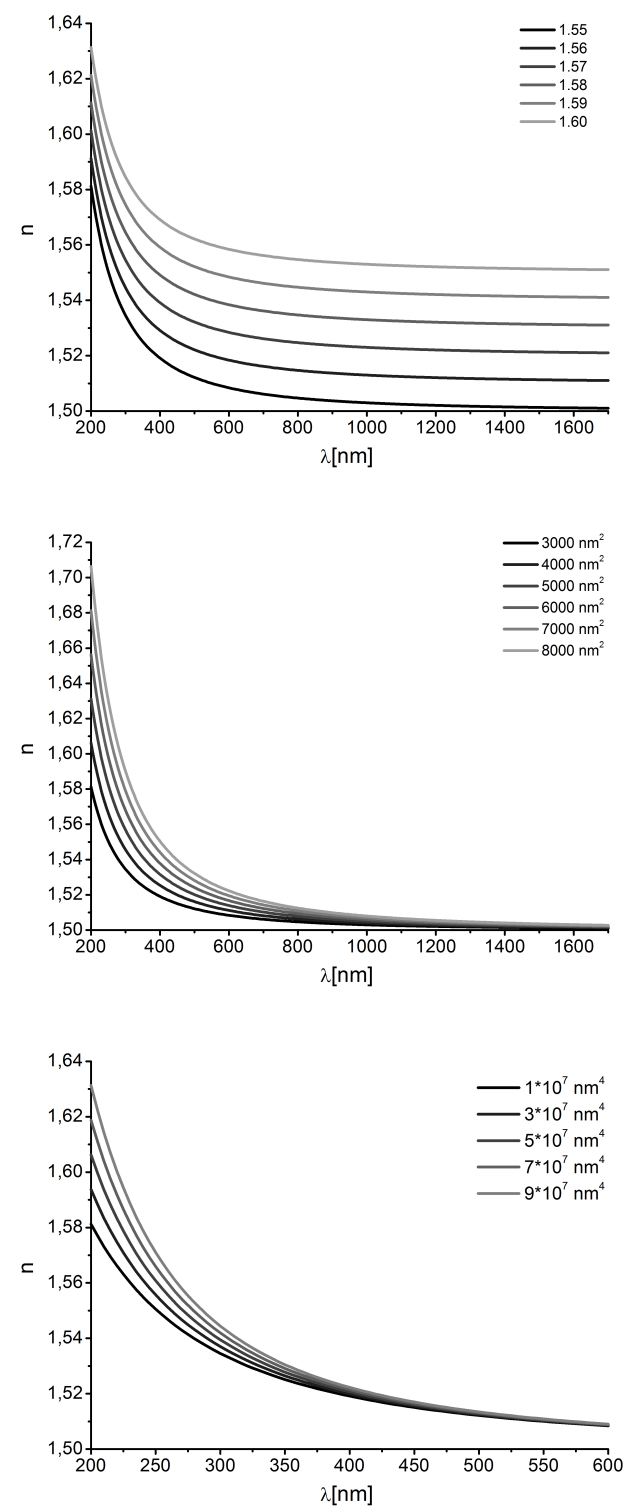

Abbildung 2.15 Dispersion nach dem CauchyModell mit Variation des Cauchy-Koeffizient (oben, $\mathrm{A}\left(A_{0}=1.5\right)$, mitte, $\mathrm{B}\left(B_{0}=3000 \mathrm{~nm}\right)$ und unten, $\left.\mathrm{C}\left(C_{0}=1 \cdot 10^{7} \mathrm{~nm}^{4}\right)\right)$.

Vereinzelt findet man in der Literatur eine analoge Beschreibungen für den Extinktionskoeffizienten $k$. 


\subsubsection{Urbach}

Das Urbach-Modell beschreibt das exponentiellen Verhalten des Extinktionskoeffizienten $\mathrm{k}$ :

$$
k(E)=U_{1} \exp \left(U_{2}\left(E-E_{\mathrm{b}}\right)\right)
$$

In diesem Modell entspricht $E_{\mathrm{b}}$ der Bandlücke des Materials. $U_{2}$ wird in der Literatur auch als Urbach-Energie bezeichnet. Sie bestimmt den exponentiellen Verlauf und ist proportional zur Temperatur. In den drei graphischen Darstellungen des Absorptionskoeffizienten (Abbildung 2.16 mit $\mathrm{U}_{1}=0.2, \mathrm{U}_{2}=0.5 \mathrm{eV}^{-1}, \mathrm{E}_{\mathrm{b}}=2 \mathrm{eV}$ ) wird jeweils einer der drei Urbach-Koeffizienten in Schritten von $0.1 \mathrm{eV}$ variiert. Zusätzlich lassen sich die Parameter $U_{1}$ und $E_{\mathrm{b}}$ direkt aus dem mittleren Plot ablesen. Die exponentielle Beschreibung des Imaginärteils taucht in vielen weiteren Modellen von Halbleitern (Tauc-Lorentz-Urbach, Cody-Lorentz-Urbach) auf. 


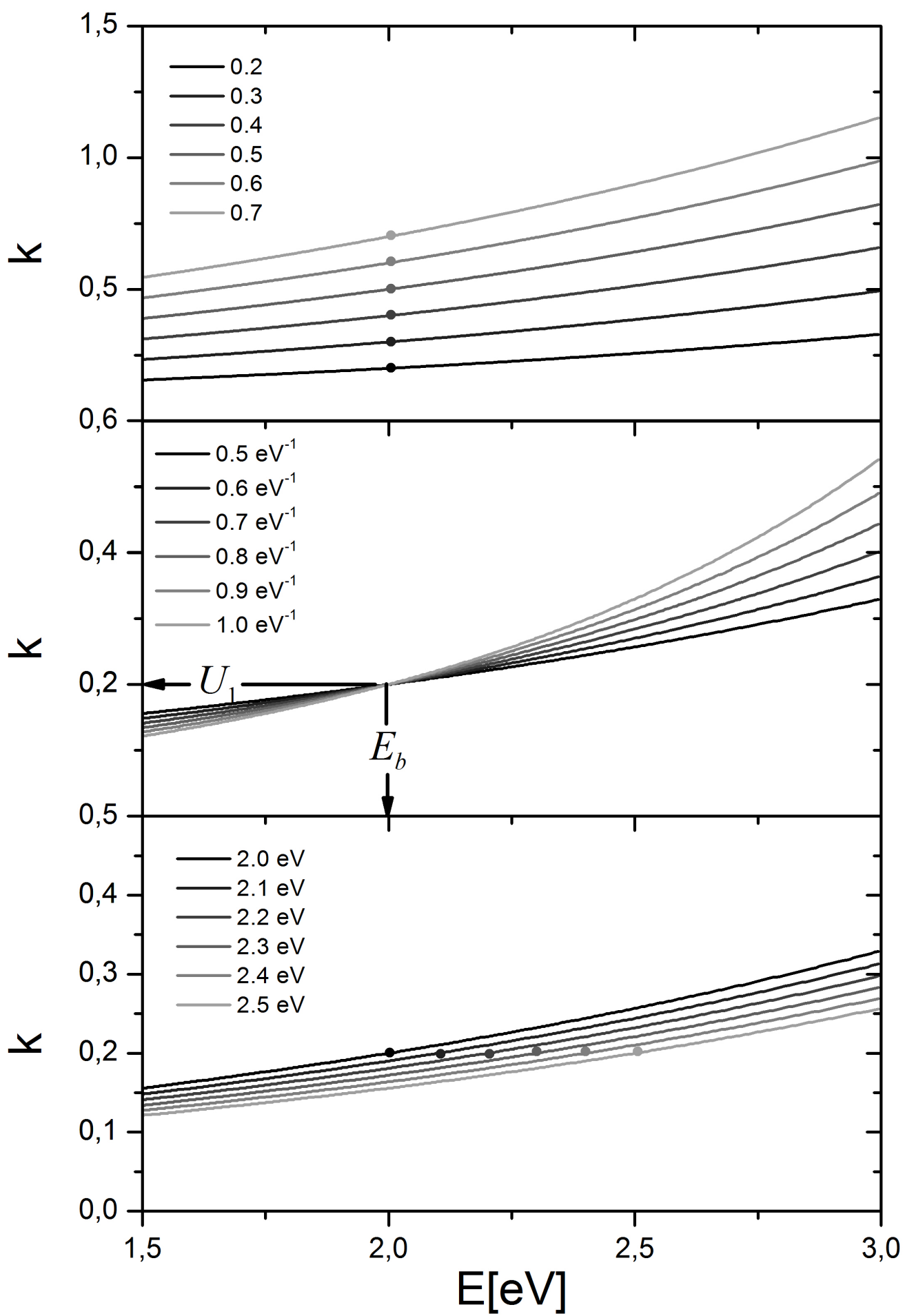

Abbildung 2.16 Energieabhängiger Verlauf des Absorptionskoeffizienten $\mathrm{k}$ nach Urbach bei verschiedenen Parametern (oben $U_{1}$, mitte $U_{2}$ und unten $E_{\mathrm{b}}$ ). 


\subsubsection{Gauss}

Der Gauss-Oszillator wird häufig bei der Beschreibung von Absorptionsbanden benutzt, die eine leichte Verbreiterung ${ }^{17}$ zeigen. Es gilt folgende mathematische Beziehung für den Imaginärteil der dielektrischen Konstante [43]:

$$
\begin{aligned}
& \varepsilon_{2}(E)=A \cdot\left[\exp \left(-\left(\frac{E-E_{\mathrm{c}}}{\sigma}\right)^{2}\right)-\exp \left(-\left(\frac{E+E_{\mathrm{c}}}{\sigma}\right)^{2}\right)\right] \\
& \sigma=\frac{B}{2 \sqrt{\ln (2)}}
\end{aligned}
$$

$A$ steht für die Amplitude des Peaks, $E_{\mathrm{c}}$ für die Resonanzenergie und $B$ für die Halbwertsbreite des Peaks. Der Realteil hat keinen analytischen Ausdruck, kann jedoch über die Kramers-Kronig-Beziehung numerisch berechnet werden. Dafür werden Stützstellen des Imaginärteils $\varepsilon_{2}$ benötigt, die das Ep4-Model bestimmt.

Ein Vergleich zwischen einem Lorentz- und Gauss-Oszillator ist in Abbildung 2.17 dargestellt.

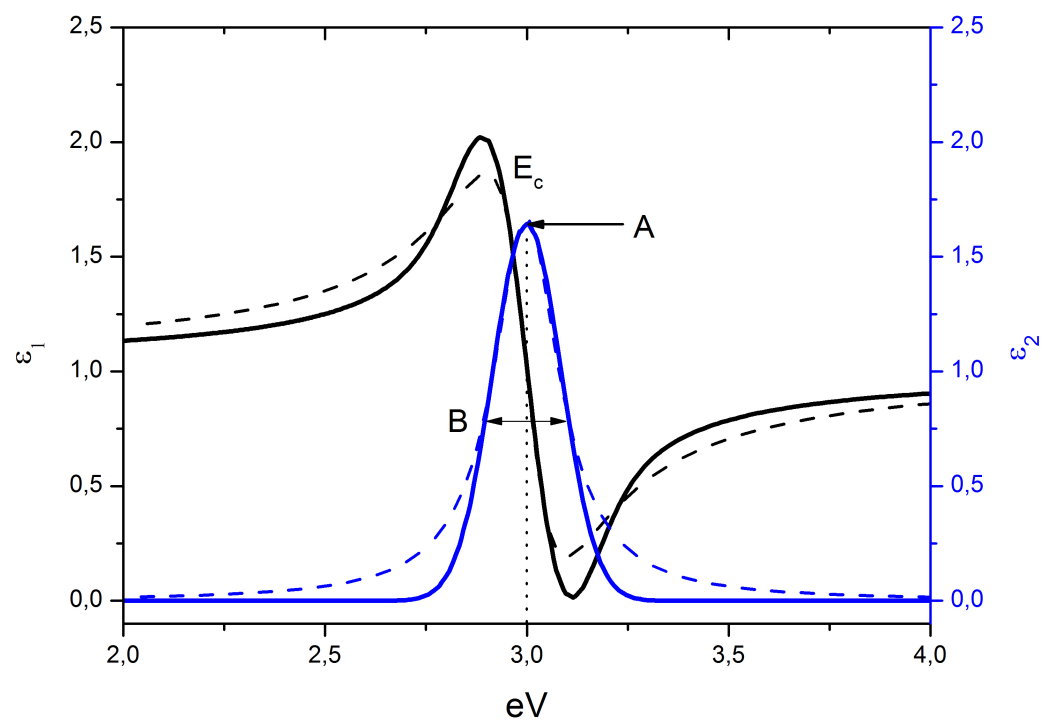

Abbildung 2.17 Graphischer Vergleich der dielektrischen Konstanten eines Gauss-Oszillators mit $E_{c}=3 \mathrm{eV}, B=0.2 \mathrm{eV}$ und $A=1.66$ in Abhängigkeit der Energie mit einem Lorentz-Oszillator.

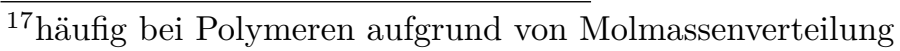


Die Halbwertsbreite $B$ des Gauss-Oszillators entspicht der Dämpfungskonstante $\Gamma$ des Lorentz-Oszillators. Die beiden anderen Parameter sind analog zum LorentzOszillator zu interpretieren. Dabei ist der Peak des Gauss-Oszillators im Imaginärteil $\varepsilon_{2}$ deutlich schmaler und nähert sich schneller der x-Achse. Im Realteil hingegen sind sowohl Minimum als auch Maximum deutlich stärker ausgeprägt im Vergleich zum Lorentz-Oszillator. Markante Punkte wie das Peak-Maximum im Imaginärteil und der Wendepunkt im Realteil liegen bei beiden Funktionen an der gleichen Stelle. Analog zum Lorentz-Oszillator wird zu dem Realteil der Wert für $\varepsilon_{0}=1$ hinzuaddiert.

\subsubsection{Drude}

Wie in Abbildung 2.13 gezeigt, liegt die Resonanzenergie des Lorentz-Oszillators für Metalle bzw. leitfähige Materialien im IR-Bereich. Die Lorentz-Funktion (Gleichung $2.37+2.38)$ wird mit $E_{0} \rightarrow 0$ zu $[55,129]$ :

$$
\begin{aligned}
& \varepsilon_{1}(E)=1-\frac{A}{\left(E^{2}+\Gamma^{2}\right)} \quad \varepsilon_{1}(\omega)=1-\frac{\omega_{\mathrm{p}}^{2}}{\left(\omega^{2}+\gamma_{\mathrm{f}}^{2}\right)} \\
& \varepsilon_{2}(E)=\frac{A \Gamma}{E\left(E^{2}+\Gamma^{2}\right)} \quad \varepsilon_{2}(\omega)=\frac{\omega_{\mathrm{p}}^{2} \gamma_{\mathrm{f}}}{\omega\left(\omega^{2}+\gamma_{\mathrm{f}}^{2}\right)}
\end{aligned}
$$

So erhält man das Drude-Modell, bei dem man von freien Elektronen im Valenzband ausgeht, die alle die gleiche Dämpfungskonstante $\gamma_{\mathrm{f}}$ besitzen. Die Plasmafrequenz $\omega_{\mathrm{p}}$ beschreibt die Konzentration der freien Elektronen und entspricht der Wurzel der Amplitude des Lorentz-Oszillators multipliziert mit dem reduzierten Wirkungsquantum $\hbar$.
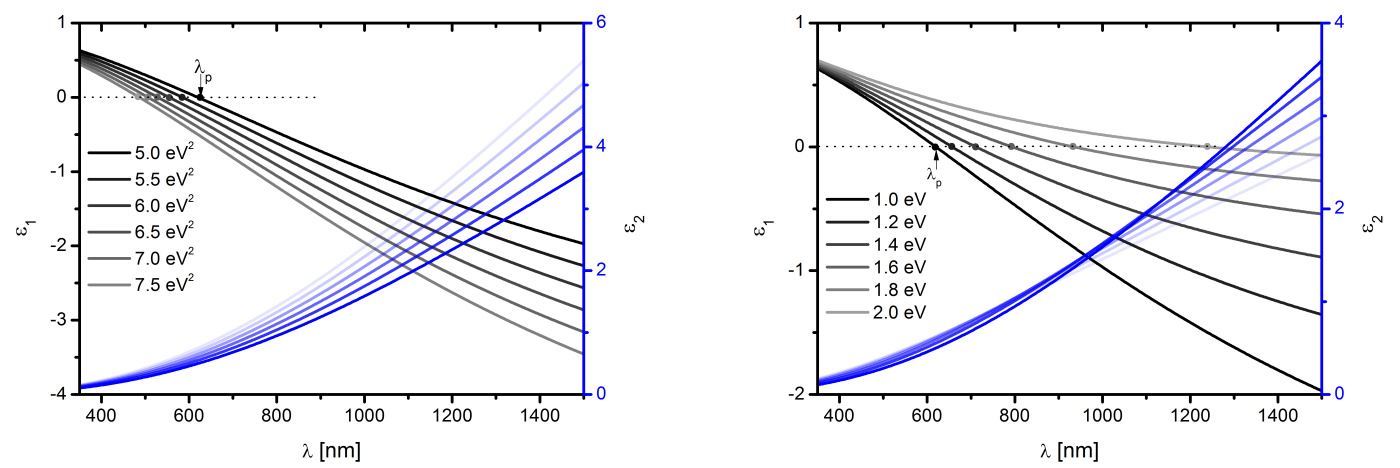

Abbildung 2.18 Dispersionsverlauf nach Drude $\left(A_{0}=5 \mathrm{eV}^{2}\right.$, $\Gamma=1 \mathrm{eV})$ bei unterschiedlichen Amplituden $A\left(+1 \mathrm{eV}^{2}\right)$ bzw. Dämpfungskonstanten $\Gamma(+0.2 \mathrm{eV})$. 
Die Abbildung 2.18 zeigt die Wellenlängenabhängigkeit der optischen Parameter nach dem Drude-Modell. Bei größeren Werten für die Amplitude (Abbildung 2.18, rechts) nimmt die negative Steigung des Realteils $\varepsilon_{1}$ bzw. die positive Steigung des Imaginärteils $\varepsilon_{2}$ zu. Diese Tatsache führt zu einer Verschiebung der Plasmawellenlänge $\lambda_{\mathrm{p}}{ }^{18} \mathrm{zu}$ höheren Energien. Nimmt die Dämpfungskonstante zu (Abbildung 2.18, links), verläuft der Realteil $\varepsilon_{1}$ erst bei höheren Wellenlängen in den negativen Bereich, was einer Verschiebung der Plasmawellenlänge zu kleineren Energien entspricht.

\subsubsection{Tauc-Lorentz, Tauc-Lorentz-Urbach und Cody-Lorentz-Urbach}

Für amorphe semitransparente Materialien (Halbleiter mit einer Bandlücke) wurde das Lorentz-Modell weiter parametrisiert, um den Übergangsbereich besser beschreiben zu können. Der Imaginärteil der Dispersion nach Tauc-Lorentz lautet [31]:

$$
\begin{aligned}
& \varepsilon_{2}(E)= \begin{cases}0 ; & E \leq E_{\mathrm{g}} \\
G_{\mathrm{T}}(E) \frac{A E_{0} \Gamma E}{\left(E_{0}^{2}-E^{2}\right)^{2}+\Gamma^{2} E^{2}} ; & E>E_{\mathrm{g}}\end{cases} \\
& G_{\mathrm{T}}(E)=\frac{\left(E-E_{\mathrm{g}}\right)^{2}}{E^{2}}
\end{aligned}
$$

In dem Modell wird ein neuer Parameter eingeführt, welcher der Bandlücke $E_{\mathrm{g}}$ entspricht. Zusätzlich wird der Imaginärteil für verschiedene Energiebereiche definiert. Bei Energien die gleich oder kleiner der Bandlücke sind, ist das Material transparent und $\varepsilon_{2}$ besitzt einen Wert von 0. Bei Energien oberhalb der Bandlücke wird der Imaginärteil als Produkt aus dem Lorentz-Term $L(E)$ und einem neu eingeführten Tauc-Term $G_{\mathrm{T}}$ berechnet. Ein Vergleich mit dem Lorentz-Oszillator zeigt, dass das Maximum im Imaginärteil $\frac{A}{\Gamma}$ entspricht und die Amplitude des Lorentz-Oszillators bei Tauc-Lorentz durch $A E_{0}$ ersetzt wurde. Die restlichen Parameter sind analog zum Lorentz-Oszillator (Kapitel 2.6.4.1). Lediglich der Tauc-Term $G_{\mathrm{T}}$ ändert den Verlauf der Dispersion. In Abbildung 2.19 ist der Verlauf der dielektrischen Konstanten nach dem Tauc-Lorentz Model $\left(A=10 \mathrm{eV}, E_{0}=5 \mathrm{eV}, \Gamma=3 \mathrm{eV}\right)$ im Bereich von $1-3 \mathrm{eV}$ bei verschiedenen Energien der Bandlücke dargestellt.

${ }^{18} \lambda_{\mathrm{p}}=\frac{c 2 \pi}{\omega_{\mathrm{p}}}$ 


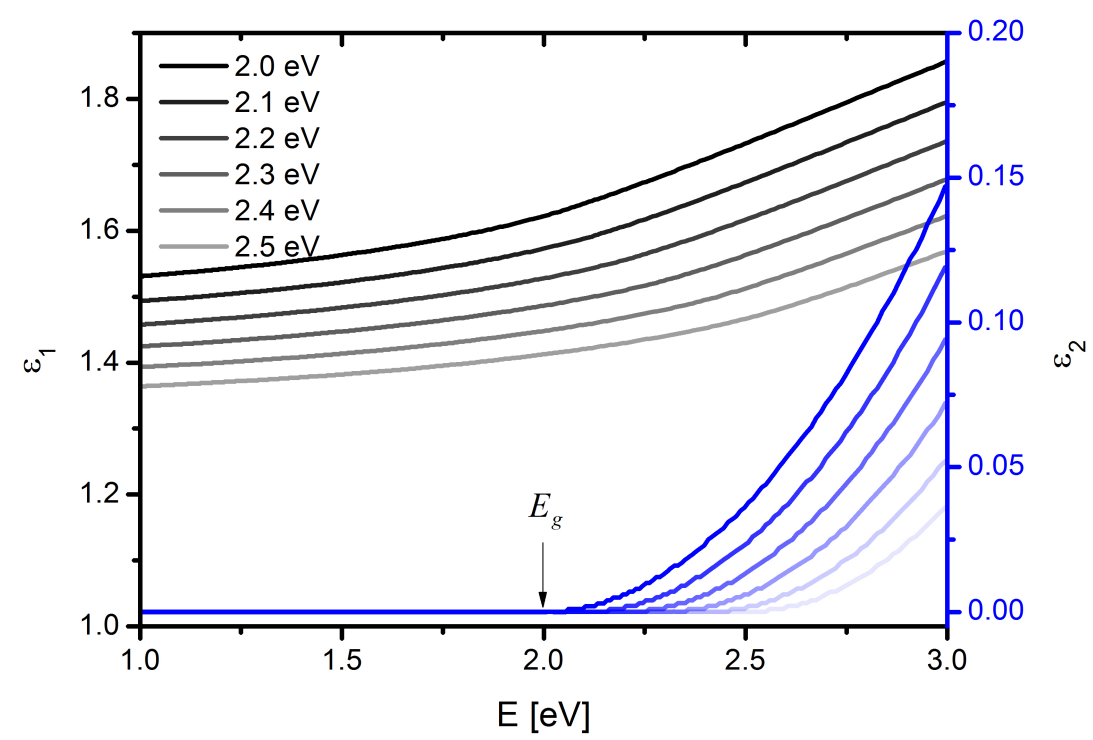

Abbildung 2.19 Dispersion nach dem Tauc-Lorentz Modell mit Varianz der Bandlücke.

In dieser Arbeit wurde zumeist das Tauc-Lorentz-Modell verwendet. Dennoch werden noch zwei weitere Modifizierungen des Tauc-Lorentz-Modells erwähnt.

Aus der Kombination des Tauc-Lorentz-Modells mit dem Urbach-Modell ergibt sich folgende Gleichung [21]:

$$
\begin{aligned}
& \varepsilon_{2}(E)= \begin{cases}\frac{E_{1}}{E} \exp \left(\frac{E-\left(E_{\mathrm{t}}+E_{\mathrm{g}}\right)}{E_{\mathrm{u}}}\right) ; & E \leq E_{\mathrm{g}} \\
G_{\mathrm{T}}(E) \frac{A E_{0} \Gamma E}{\left(E_{0}^{2}-E^{2}\right)^{2}+\Gamma^{2} E^{2}} ; & E>E_{\mathrm{g}}\end{cases} \\
& E_{1}=\left(E_{\mathrm{t}}+E_{\mathrm{g}}\right) L\left(E_{\mathrm{t}}+E_{\mathrm{g}}\right) G_{\mathrm{T}}\left(E_{\mathrm{t}}+E_{\mathrm{g}}\right)
\end{aligned}
$$

Dabei ist $E_{\mathrm{t}}$ ein Parameter, der einer Offset-Energie zur Bandlücke $E_{\mathrm{g}}$ entspricht. Die graphische Darstellung der dielektrischen Konstanten nach dem Tauc-LorentzUrbach Modell $\left(A=10 \mathrm{eV}, E_{0}=5 \mathrm{eV}, \Gamma=3 \mathrm{eV}, E_{\mathrm{g}}=2 \mathrm{eV}\right)$ bei verschiedenen Werten für $E_{\mathrm{t}}$ und $E_{\mathrm{u}}$ wird in Abbildung 2.6.4.6 gezeigt.

Im Cody-Lorentz-Urbach Modell wird ein neuer Parameter $E_{\mathrm{p}}$ dem Tauc-Term $G_{\mathrm{T}}$ hinzugefügt. So erhält man für den Cody-Term $G_{C}$, der den Tauc-Term ersetzt:

$$
\begin{aligned}
& G_{\mathrm{C}}(E)=\frac{\left(E-E_{\mathrm{g}}\right)^{2}}{\left(E-E_{\mathrm{g}}\right)^{2}+E_{\mathrm{p}}^{2}} \\
& E_{1}=\left(E_{\mathrm{t}}+E_{\mathrm{g}}\right) L\left(E_{\mathrm{t}}+E_{\mathrm{g}}\right) G_{C}\left(E_{\mathrm{t}}+E_{\mathrm{g}}\right)
\end{aligned}
$$



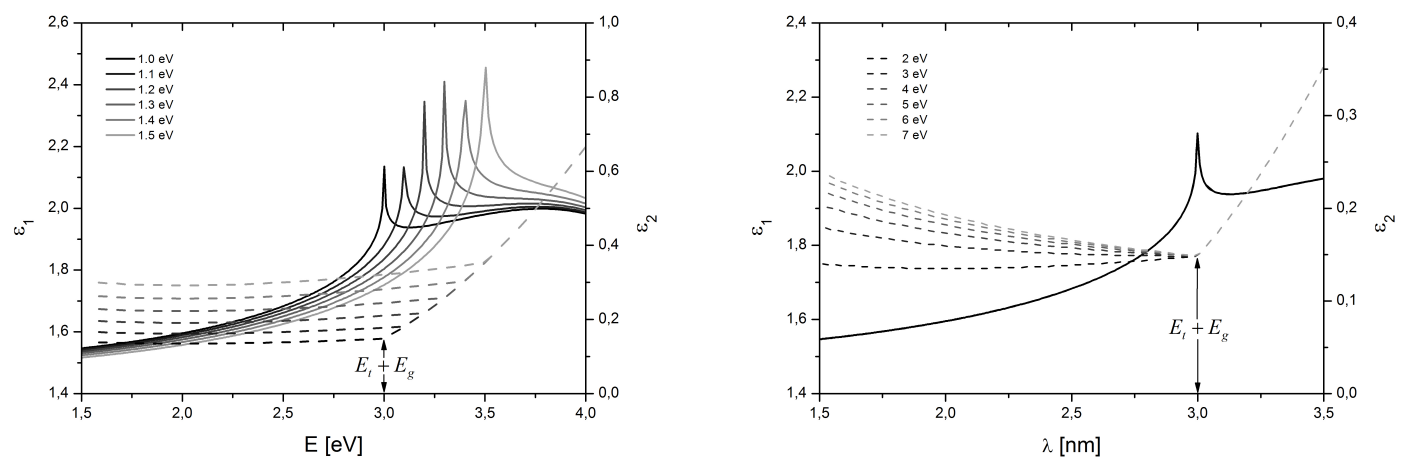

Abbildung 2.20 Dispersion nach dem Tauc-Lorentz-UrbachModell bei verschiedenen Werten für $E_{\mathrm{t}}$ und $E_{\mathrm{u}}$.

Der Imaginärteil entspricht ansonsten dem Tauc-Lorentz-Urbach-Modell (siehe Gleichung 2.49).

\subsubsection{Effektives Medium Theorie}

In diesem Unterabschnitt werden nicht ideale Schichtsysteme betrachtet, sondern Effekte wie Rauigkeit, Materialmischungen oder Grenzschichten als physikalisches Modell beschrieben [6].

Es gelten folgende Voraussetzungen [22]:

- Die Größe der gemischten Phasen darf einerseits nicht atomarer Natur und anderseits nicht größer als $1 / 10$ der Wellenlänge sein

- Die optischen Parameter der Phasen sind unabhängig bezüglich Größe und Form

Grundlegend unterscheidet man, ob es sich um eine sphärische oder laminare Anordnung handelt.

\subsubsection{Sphärische Mikrostruktur}

Bei Mischungen (sphärisch, symmetrisch) von zwei Materialien gilt folgender allgemeiner Zusammenhang [22, 178][55, 260]:

$$
\frac{\varepsilon-\varepsilon_{\mathrm{h}}}{\varepsilon+2 \varepsilon_{\mathrm{h}}}=f_{\mathrm{a}} \frac{\varepsilon_{\mathrm{a}}-\varepsilon_{\mathrm{h}}}{\varepsilon_{\mathrm{a}}+2 \varepsilon_{\mathrm{h}}}+\left(1-f_{\mathrm{a}}\right) \frac{\varepsilon_{\mathrm{b}}-\varepsilon_{\mathrm{h}}}{\varepsilon_{\mathrm{b}}+2 \varepsilon_{\mathrm{h}}}
$$

Der Parameter $f_{\mathrm{a}}$ wird als Fraktionsfaktor eingeführt. Die dielektrischen Konstanten der beiden Materialien A und B werden durch die jeweiligen Indizes repräsentiert. Der Koeffizient $\varepsilon_{\mathrm{h}}$ entspricht dem jeweiligen Medium und wird je nach Modell ersetzt. Die allgemeine Form vereinfacht sich. 
- Basierend auf der Claussius-Mossotti-Gleichung und mit der Annahme das $\varepsilon_{\mathrm{h}}=1$ entspricht, ergibt sich für Gleichung 2.53:

$$
\frac{\varepsilon-1}{\varepsilon+2}=f_{\mathrm{a}} \frac{\varepsilon_{\mathrm{a}}-1}{\varepsilon_{\mathrm{a}}+2}+\left(1-f_{\mathrm{a}}\right) \frac{\varepsilon_{\mathrm{b}}-1}{\varepsilon_{\mathrm{b}}+2}
$$

- Davon ausgehend, dass die Hauptkomponente Material A ist und Material B kugelförmig eingebettet ist, wobei $\varepsilon_{\mathrm{h}}=\varepsilon_{\mathrm{a}}$ entspricht, resultiert aus Gleichung 2.53 die Maxwell-Garnet-Gleichung:

$$
\frac{\varepsilon-\varepsilon_{\mathrm{a}}}{\varepsilon+2 \varepsilon_{\mathrm{a}}}=\left(1-f_{\mathrm{a}}\right) \frac{\varepsilon_{\mathrm{b}}-\varepsilon_{\mathrm{a}}}{\varepsilon_{\mathrm{b}}+2 \varepsilon_{\mathrm{a}}}
$$

- Werden beide Komponenten als gleichwertig betrachtet, gilt $\varepsilon_{\mathrm{h}}=\varepsilon$ woraus die Beschreibung eines effektiven Mediums nach Bruggeman resultiert:

$$
0=f_{\mathrm{a}} \frac{\varepsilon_{\mathrm{a}}-\varepsilon}{\varepsilon_{\mathrm{a}}+2 \varepsilon}+\left(1-f_{\mathrm{a}}\right) \frac{\varepsilon_{\mathrm{b}}-\varepsilon}{\varepsilon_{\mathrm{b}}+2 \varepsilon}
$$

Ein direkter Vergleich der drei Theorien ist in Abbildung 2.21 dargestellt. Hier wird $\varepsilon$ in Abhängigkeit von $f_{\mathrm{a}}$ nach den jeweiligen Modellen gezeigt.

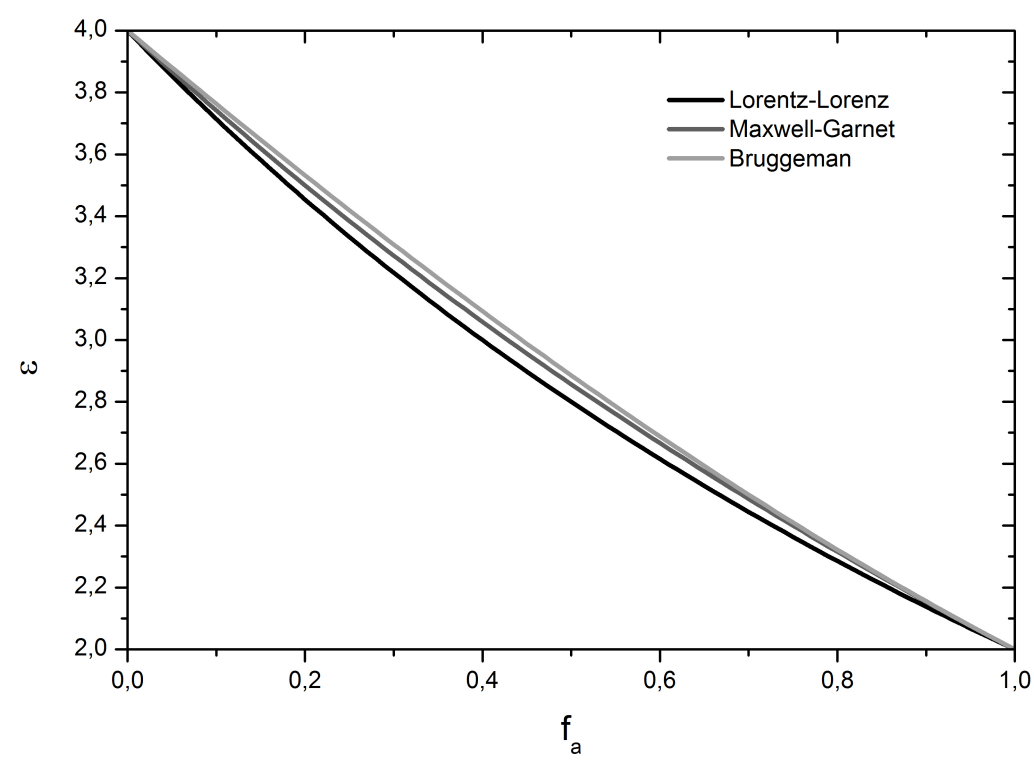

Abbildung 2.21 Sphärische Mikrostruktur: ein Vergleich der drei Theorien.

\subsubsection{Laminare Mikrostruktur}

Folgende Gleichung gilt allgemein für laminare Zweiphasen-Materialien [6]:

$$
\varepsilon=\frac{\varepsilon_{\mathrm{a}} \varepsilon_{\mathrm{b}}+\kappa \varepsilon_{h}\left(f_{\mathrm{a}} \varepsilon_{\mathrm{a}}+\left(1-f_{\mathrm{a}}\right) \varepsilon_{\mathrm{b}}\right)}{\kappa \varepsilon_{h}+\left(f_{\mathrm{a}} \varepsilon_{\mathrm{a}}+\left(1-f_{\mathrm{a}}\right) \varepsilon_{\mathrm{b}}\right)}
$$


mit

$$
\kappa=\frac{(1-q)}{q}
$$

wobei ein neuer Parameter q eingeführt wird, der in der Literatur als screening factor bezeichnet wird. Für Mikrostrukturen, deren Lamellen senkrecht zur Probenoberfläche verlaufen, wird $\mathrm{q}=0$ und Gleichung 2.57 vereinfacht sich zu:

$$
\varepsilon=f_{\mathrm{a}} \varepsilon_{\mathrm{a}}+\left(1-f_{\mathrm{a}}\right) \varepsilon_{b}
$$

Die daraus resultierende optische Konstante setzt sich linear aus den beiden Komponenten zusammen. Zeigt die Schicht des Zweiphasen-Materials einen laminaren Verlauf parallel zur Probenebene, wird $q=1$ und die resultierende Dielektrizitätskonstante setzt sich wie folgt zusammen:

$$
\frac{1}{\varepsilon}=\frac{f_{\mathrm{a}}}{\varepsilon_{\mathrm{a}}}+\frac{\left(1-f_{\mathrm{a}}\right)}{\varepsilon_{b}}
$$

Der Parameter q kann Werte von 0 bis 1 annehmen. Bei sphärischer Anordnung der zwei Medien entspricht der Wert $\frac{1}{3}$. Abbildung 2.22 zeigt den Einfluss des screening factors $\mathrm{q}$ auf $\varepsilon$ bei variierendem Fraktionsfaktor $f_{\mathrm{a}}$.

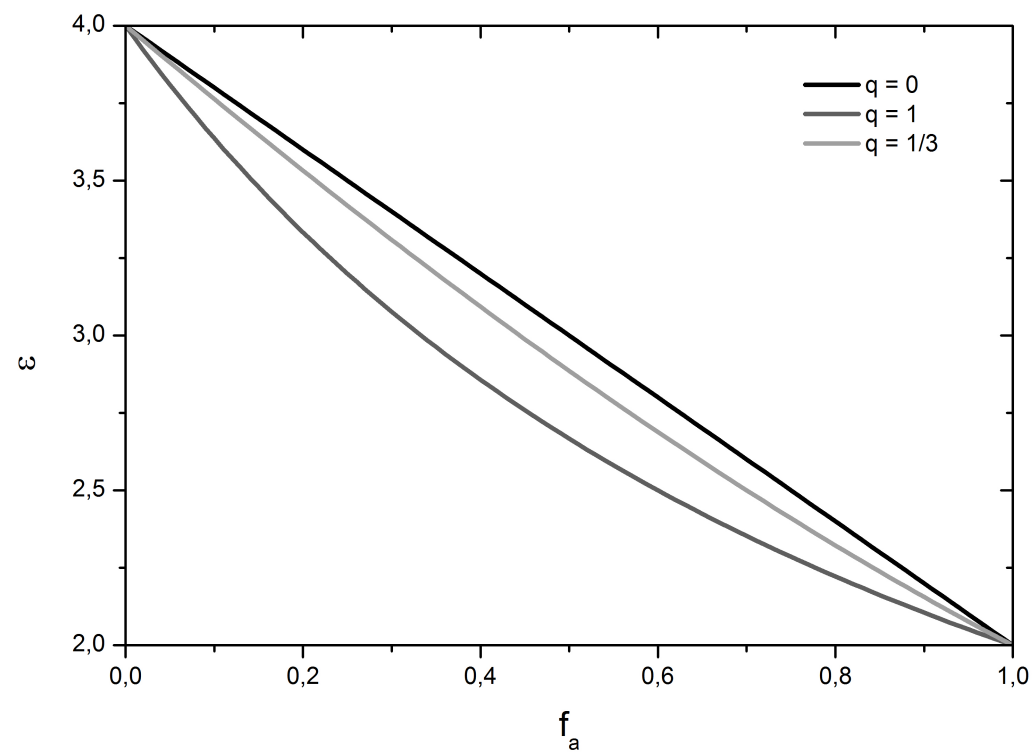

Abbildung 2.22 Laminare Mikrostruktur. 


\section{Methoden}

In dieser Arbeit wurden neben der abbildenden Nulling Ellipsometrie, als zentrale Messtechnik, auch andere Technologien im Bereich der Oberflächenanalytik verwendet, die im folgenden Kapitel genauer erläutert werden.

\subsection{Ellipsometrie}

Nachdem im Kapitel 2 auf die physikalischen Grundlagen der Ellipsometrie eingegangen wurde, behandelt das folgende Kapitel Ellipsometrie als optisches Messverfahren im Bereich der Oberflächenanalytik. Der Fokus dieses Kapitels liegt auf dem Aspekt abbildender Nulling Ellipsometrie und dem prinzipiellen Aufbau des abbildenden Ellipsometers, das in dieser Arbeit verwendet und weiterentwickelt wurde.

Der Vorteil der Ellipsometrie, als nicht-destruktive, kontaktfreie Messmethode, ist die hohe Präzision und Sensitivität die Schichtdicke betreffend (bis zu $0.1 \AA$ ). Als weitere Option können die optischen Parameter der Medien bestimmt werden. Nachteilig ist jedoch, dass es sich um eine indirekte Messmethode handelt, bei der erst durch geeignete Modellierung die gewünschten Parameter ermittelt werden können. Somit ist neben der Aufnahme von Messdaten der Ellipsometrie besonders deren Auswertung einer der Schlüsselfaktoren für den Anwender [22].

\subsubsection{Messmodi}

Wie bereits im Kapitel 2.5 genauer erläutert wurde, sind die ellipsometrischen Parameter abhängig von der Wellenlänge $\lambda$ und dem Einfallswinkel $\phi_{\mathrm{i}}$, die als bekannte Größen variiert werden können. Je nachdem, welche messtechnisch bekannte Größe variiert wird, spricht man von Einwellenellipsometrie (Single Wavelength Ellipsometry SWE) oder Spektroskopischen Ellipsometrie (Spectroscopic Ellipsometry SE), auf die im nächsten Unterkapitel genauer eingegangen wird.

\subsubsection{Einwellenellipsometrie}

Die ersten Ellipsometer wurden mit einer monochromatischen Lichtquelle betrieben. Zusätzlich vereinfachte der Aspekt der singulären Wellenlänge das Modellieren der Messdaten. Anhand von Winkelspektren können neben der Schichtdicke nur die optischen Konstanten für die jeweilig genutzte Wellenlänge bestimmt werden.

Eine der wichtigsten Voraussetzungen, um die Schichtdicke anhand der ellipsometrischen Parameter zu bestimmen, ist neben der Planarität und Isotropie des Sub- 
strats $^{19}$, dass es sich um eine im Messbereich transparente Schicht handelt. Wie in Abbildung $3.1 \mathrm{zu}$ sehen, wiederholen sich die Werte für Delta und Psi ab einer gewissen Schichtdicke bei transparenten Materialien. Deshalb wird zusätzlich der Messwinkel und/oder die Wellenlänge variiert, um somit die tatsächliche Schichtdicke zu bestimmen.
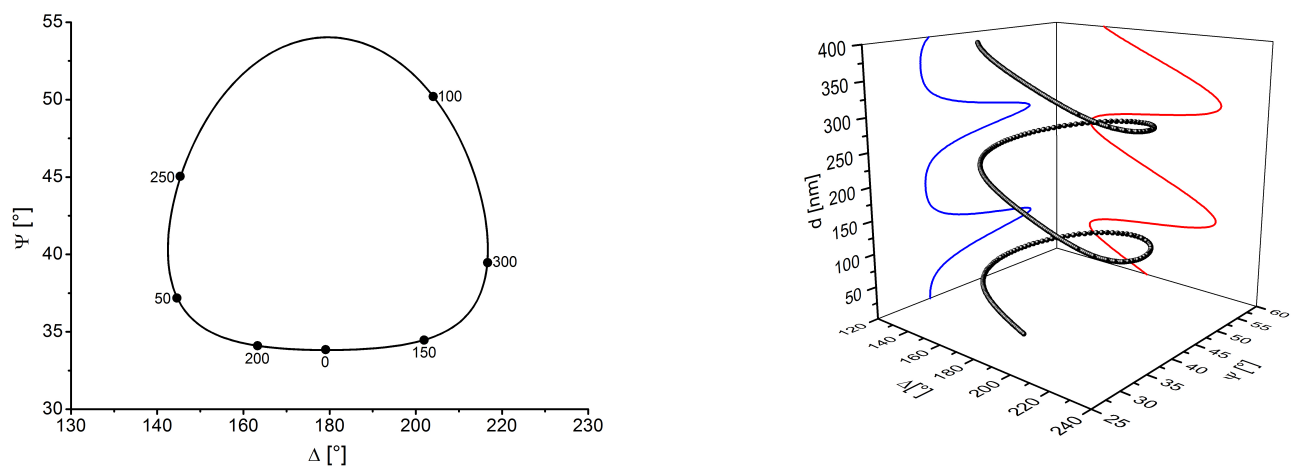

Abbildung 3.1 Delta-Psi vs. Schichtdicke von $\mathrm{SiO}_{2}(\lambda=658 \mathrm{~nm})$ als transparente Schicht auf Si (100).

Handelt es sich um eine absorbierende Schicht (wie bspw. Metall oder Halbleiter), können nur dünne Schichten bestimmt werden. Die ellipsometrischen Parameter konvergieren in Abhängigkeit der Schichtdicke einem konstanten Wert (Abbildung 3.2). Dieser Effekt wird häufig genutzt um von intransparenten Materialien direkt die optischen Parameter mittels Konvertierung („bulk“) zu generieren. Besonders bei metallischen Schichten ist zu beachten, dass die optischen Konstanten von dünnen Filmen nicht den Werten von „,bulk“ Materialien entsprechen.
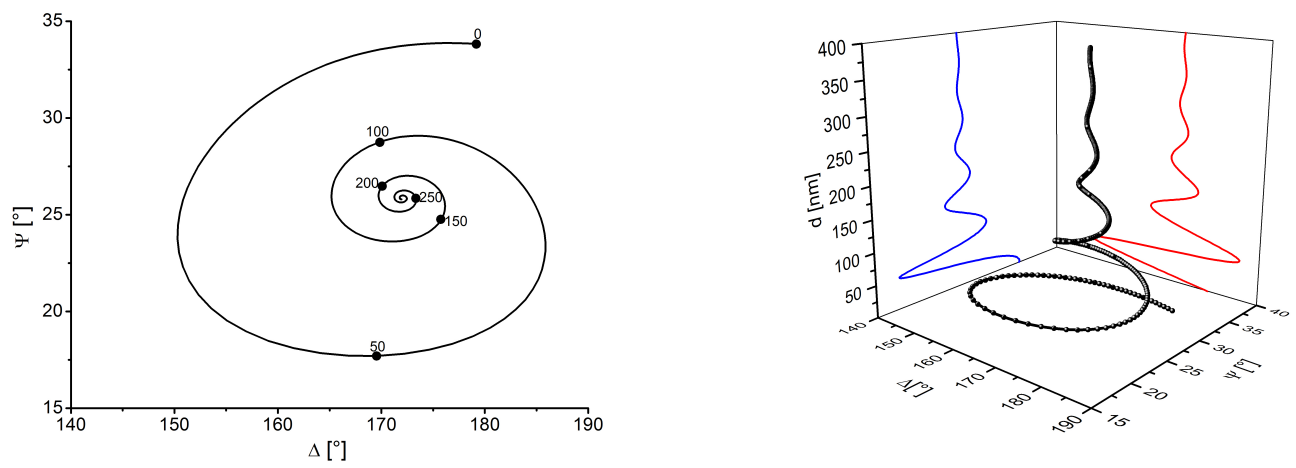

Abbildung 3.2 Delta-Psi vs. Schichtdicke von $\operatorname{SiO}_{\mathrm{x}}(\lambda=658 \mathrm{~nm})$ als semi-transparente Schicht auf Si (100).

Messtechnisch wird für jeden Einfallswinkel ein Delta/Psi-Paar bestimmt, wodurch sich ein Winkelspektrum (Abbildung 3.3) ergibt. Als monochromatische Lichtquelle werden häufig Laser eingesetzt, die eine schmale Bandbreite besitzen. Der limitierende Faktor der Winkelspektren ist die Genauigkeit des Goniometers. Zusätzlich

${ }^{19}$ Gilt für die Nulling Ellipsometrie 
zu dünnen Schichten können mit hochaufgelösten AOI-Spektren Schichten bis über $10 \mu \mathrm{m}$ bestimmt werden.

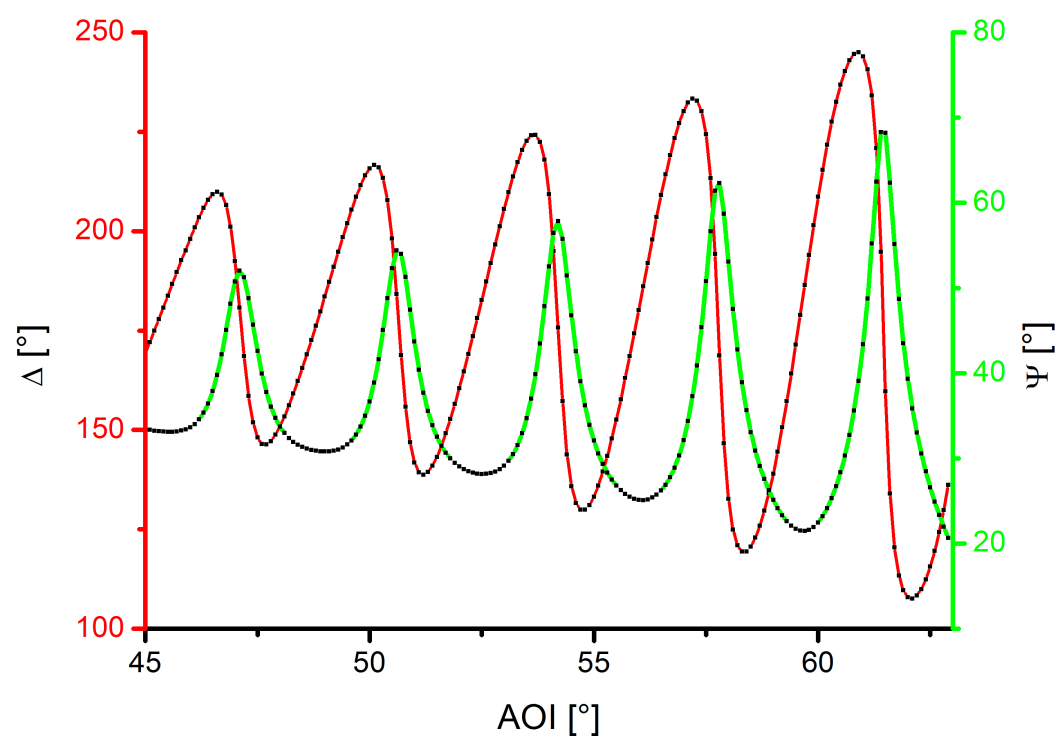

Abbildung 3.3 Hochaufgelöstes Winkelspektrum eines transparenten Polymerfilms ( $\mathrm{d}=14042 \mathrm{~nm}, \mathrm{n}=1.4136)$ auf $\mathrm{Si}$ (100) bei $\lambda=658 \mathrm{~nm}$.

In Abbildung 3.3 sieht man ein hochaufgelöstes AOI-Spektrum eines Polymerenfilms auf Si (100) bei einer Wellenlänge von $658 \mathrm{~nm}$. Die Daten wurden in einem Winkelbereich von $45-63^{\circ}$ in $0.1^{\circ}$-Schritten aufgenommen.

Die Datenanalyse liefert Werte für die Schichtdicke $(\mathrm{d}=14042 \pm 4 \mathrm{~nm})$ und die optischen Parameter $\left(\mathrm{n}\left(\lambda_{658} \mathrm{~nm}\right)=1.4136 \pm 0.0003\right)$ des Materials. In diesem Beispiel handelte es sich um ein transparentes Material $(\mathrm{k}=0)$. Bei semitransparenten Materialien können $n$ und k-Werte der jeweiligen Wellenlänge bestimmt werden. Ob aus den Daten ebenfalls die Schichtdicke des Materials bestimmt werden kann, ist wie bereits erwähnt von Schichtdicke und dem Ausmaß an Absorption abhängig.

\subsubsection{Spektroskopische Ellipsometrie}

Im Gegensatz zum Winkelspektrum wird bei spektroskopischen Messungen der Messwinkel konstant gehalten und die genutzte Wellenlänge variiert. Wie in Abbildung 3.4 gezeigt, ergibt sich daraus ein Wellenlängenspektrum von Delta und Psi bei einem bestimmten AOI. Anhand einer geeigneten Auswertung mittels Modellierung (siehe Kapitel 3.1.3.4) erhält man neben der Schichtdicke ebenfalls die wellenabhängigen optischen Parameter (Dispersion). Bei einer Probe, bei der es sich nur um das reine Substrat handelt („,bulk“ Material), lassen sich anhand der direkten Konvertierung von Delta und Psi die dielektrischen Eigenschaften mathematisch berechnen. Der Vorteil der spektroskopischen Messung im Vergleich zu den Einwellenmessung besteht darin, dass die Schichtdicke transparenter Materialien eindeutiger bestimmt 
werden kann. Charakteristische Merkmale in der Dispersion der Medien können zusätzlich bestimmt werden.
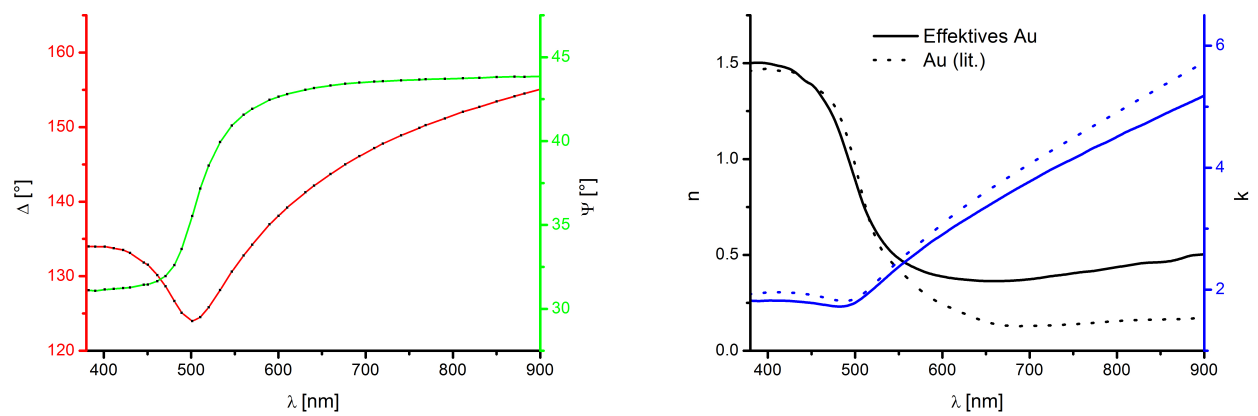

Abbildung 3.4 Spektroskopische Messung an einem intransparenten Gold-Film und deren Dispersion im Vergleich zur Datenbank.

Die linke Seite der Abbildung 3.4 zeigt eine spektroskopische Messung eines intransparenten Gold-Films auf Glas. Der Einfallswinkel beträgt $55^{\circ}$ und es wurden in $20 \mathrm{~nm}$-Schritten im Wellenlängenbereich von 380-900 nm Daten aufgenommen. Auf der rechten Seiten sieht man die durch direkte Konvertierung erhaltenen optischen Parameter des intransparenten Gold-Films im Vergleich zur Dispersion von Gold aus der Literatur [33]. Es ist ein deutlicher Unterschied zwischen den beiden Dispersionen zu erkennen, der sich durch die unterschiedlichen Herstellungsprozesse und eventuelle Rauheit des Films erklären lässt.

Häufig findet man in Veröffentlichungen spektroskopische Messungen, die bei mehreren Einfallswinkeln aufgenommen wurden. Solche Messungen werden auch als VASE (variable angle spectroscopic ellipsometry) Messung bezeichnet. Diese Datensätze liefern besonders viele optische Informationen über das Probensystem. Bei der Datenanalyse können mehrere Parameter gleichzeitig bestimmt werden, ohne das System bezüglich der Variablen überzubestimmen.

\section{Kinetik}

Ellipsometrie kann auch in-situ durchgeführt werden. Je nachdem, wie schnell die Daten eines Ellipsometers generiert werden können, wird entweder das Wellenlängenspektrum oder nur eine bestimmte Wellenlänge genutzt, um kinetische Prozesse zu detektieren. Der Einfallsswinkel wird häufig konstant gehalten und Prozesse, wie beispielsweise Absorption/Desorption von Proteinen [19], Temperaturverhalten von Polymeren [46], Abscheidevorgänge [36] uvm. werden in Echtzeit verfolgt. Der limitierende Faktor bei kinetischen Messungen ist die Rotation der optischen Komponenten. Bei der Off-Null-Ellipsomtrie können jedoch aufgrund des Ausbleibens der Rotation der optischen Komponenten kinetische Vorgänge im Bereich von Millisekunden durchgeführt werden [5].

Bei Absorption von Proteinen oder Abscheideprozessen wird eine Schichtdickenzunahme detektiert (Abbildung 3.5). 

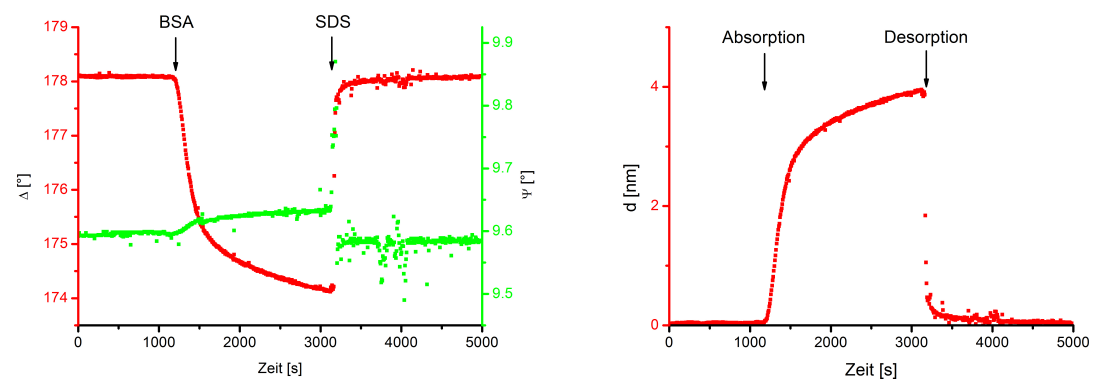

\begin{abstract}
Abbildung 3.5 Absorption und Desorption von Protein (bovine serum albumin, BSA) an hydrophilisierter $\mathrm{SiO}_{2}$ Oberfläche mit den ellipsometrischen Messdaten (links) und der berechneten Proteinschichtdicke (rechts).
\end{abstract}

Physikalische Phänome wie Phasenübergänge von Lipiden [20, 50], pH-Abhängikeit [61] oder Quellverhalten von Polymeren [39, 49] können ebenfalls messteschnisch verfolgt werden. Neben einer Schichtdickenänderung kommt es zusätzlich zu einer Änderung der optischen Eigenschaften des Materials.

Diese Arbeit fokussiert sich auf temperaturabhängige Messungen an Polymeren und der Implementierung der Heizzelle ins System.

\title{
3.1.2 Ellipsometriearten
}

In diesem Unterkapitel wird genauer erläutert wie man messtechnisch die ellipsometrischen Daten generiert. Es wird grundsätzlich zwischen mehren Ellipsometriearten differenziert. Diese Arbeit fokussiert sich auf die Nulling Ellipsometrie. Die sonstigen Ellipsometriearten wie Rotating Element Ellipsometry (Rotating Polarizer Ellipsometry RPE, Rotating Analyzer Ellipsometry RAE), Rotating Compensator Ellipsometry (RCE), Polarization Modulation Ellipsometry (PME) und Multichannel Ellipsometry (ME) seien der Vollständigkeit halber erwähnt, einen tieferen Einblick gewährt die einschlägige Literatur „Spectroscopic ellipsometry“ von H. FuJIWARA [22] sowie „Handbook of ellipsometry" von H. Tompkins und E. A. Irene[55].

\subsubsection{Nulling Ellipsometrie}

Als eine der ältesten Formen der Ellipsometrie, die schon von Paul Drude genutzt wurde, werden die ellipsometrischen Parameter $\Delta$ und $\Psi$ direkt anhand der Position („Nulling Condition“") der optischen Komponenten (Polarisator und Analysator) bestimmt. Der prinzipielle Aufbau eines Nulling Ellipsometers in PCSA (PolarizerCompensator-Sample-Analyzer) Konfiguration ist in Abbildung 3.6 dargestellt.

Wie auch bei allen anderen Ellipsometern besteht das Gerät aus einer Lichtquelle, optischen Komponenten, der zu untersuchenden Probe und einem Detektor. Bei den optischen Komponenten handelt es sich um zwei lineare Polarisatoren (Polarisator $P$ und Analysator $A$ ) und ein $\lambda / 4$-Plättchen (Kompensator $C$ ). Bei der Nulling 


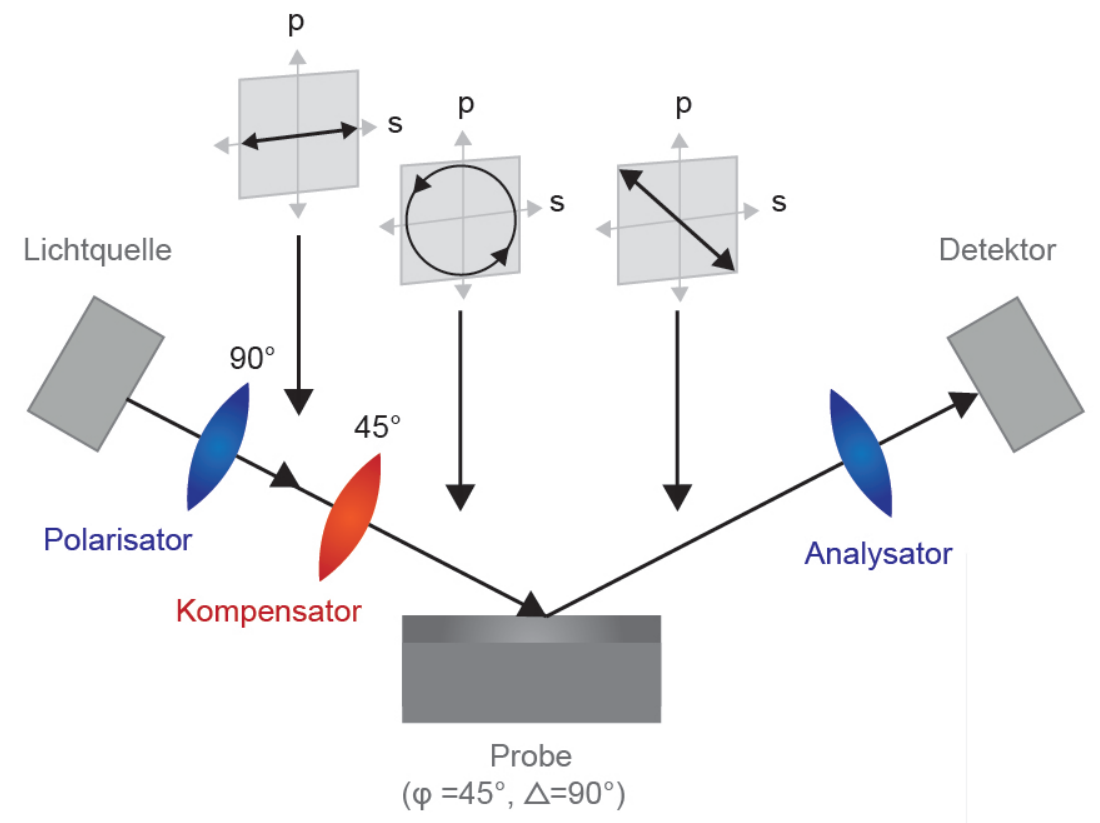

\begin{abstract}
Abbildung 3.6 Schematische Abbildung eines Nulling Ellipsometers in PCSA-Kinfiguration inklsuive Darstellung des Polarisationszustandes des Lichtstrahls.
\end{abstract}

Ellipsometrie wird monochromatisches Licht verwendet, das nach Transmission des Polarisators linear polarisiert und anschließend durch Passieren des Kompensators elliptisch wird. Wie im vorherigen Kapitel 2.3 beschrieben, ändert sich nach Reflektion an der Grenzfläche der Probe der Polarisationszustand des Lichts und wird nach Transmission durch den Analysator wieder linear. Ziel ist es, den Polarisator und den Analysator so einzustellen, dass es am Detektor bestenfalls zu einer Auslöschung des Signals (Null) am Detektor kommt: das sogenannte „Nulling“-Prinzip. Dazu muss der Polarisator so eingestellt sein, dass das an der Probe reflektierte Licht linear polarisiert ist und es anschließend durch eine gekreuzte Stellung des Analysators (um $90^{\circ}$ versetzt) zur Auslöschung kommt. Meistens findet man im detektierten Intensitätssignal beim Einstellen von Polarisator und Analysator lediglich ein Minimum. Unter den „Nulling Conditions“ mit einem idealen $\lambda / 4$-Plättchen werden die ellipsometrischen Parameter anhand der Stellung von P und A wie folgt bestimmt $[55,13]$ :

$$
\Psi=\left|\mathrm{A}_{0}\right|, \quad \Delta=2 \mathrm{P}_{0}+\frac{\pi}{2}
$$

mit

$$
C= \pm 45^{\circ}
$$


Dabei entsprechen $P_{0}$ und $A_{0}$ den jeweiligen Positionen des Polarisators bzw. Analysators - bezogen auf die Einfallsebene -, bei denen die "Nulling Conditions" erfüllt werden.

Der Vorgang des "Nullings" wird heutzutage von der Bediensoftware automatisch durchgeführt. Dabei wird zunächst der Polarisator rotiert und das Intensitätssignal entsprechend der Polarisatorposition detektiert. An den Datenpunkten wird anschließend ein geeigneter Fit angelegt und das Intensitätsminimum bestimmt $\left(P_{\min }\right)$. Der Anwender bestimmt durch die Anzahl der aufgenommenen Datenpunkte und die Länge des Verfahrwegs des Polarisators aus der Ist-Position (ranges $\left[^{\circ}\right]$ ), wie exakt das Minimum bestimmt wird. Der Polarisator wird auf die Minimumposition gesetzt und anschließend wird der Vorgang analog mit dem Analysator durchgeführt.

Der ganze Vorgang sollte bestenfalls iterativ so lange wiederholt werden, bis die Stellung der optischen Komponenten der absoluten Minimumposition konvergieren $[7,384]$. Es existieren 4 Zonen, in denen die „Nulling“-Kriterien erfüllt sind [7, 204]. Um gerätespezifische Fehler (Achsenshift und Retardierung des Kompensators, etc.) zu minimieren, werden die ellipsometrischen Werte über die 4 Zonen gemittelt. Sowohl der Aspekt der Iteration als auch der 4 Zonen ist ein wichtiger Faktor bei der Messgeschwindigkeit und wurde im Rahmen dieser Arbeit verbessert (Kapitel 4.3).

\subsubsection{Abbildende Nulling Ellipsometrie}

Bei dem in dieser Arbeit genutzten Ellipsometer handelt es sich um ein abbildendes auto-nulling Ellipsometer (Accurion GmbH). Es gibt mehrere mögliche Konfigurationen, wie ein abbildendes Ellipsometer aufgebaut sein kann. Prinzipiell wird zusätzlich ein Objektiv bzw. eine Linse in den Strahlengang eingeführt und der Detektor durch eine CCD-Kamera (charge couple device) ersetzt. Dies ermöglicht, ortsaufgelöst die ellipsometrischen Messungen durchzuführen. Im Vergleich zu allen nicht abbildenden Systemen gibt es zwei Aspekte, die für den Anwender zu beachten sind.

Abbildende Ellipsometer besitzen, einerseits, eine deutlich bessere laterale Auflösung im Vergleich zu nicht abbildenden Systemen, bei denen die Größe des Lichtspots den begrenzenden Faktor darstellt. Je nach Position des Linsensystems im Strahlengang - vor oder nach dem Analysator -, kann eine laterale Auflösung von $1 \mu \mathrm{m}$ erreicht werden. Andererseits ist die Messzeit im Vergleich zu herkömmlichen, nicht abbildenden Geräten deutlich länger, da das Ellipsometer dispersiv betrieben wird. Alle sonstigen Vor- und Nachteile gelten für abbildende Ellipsometer und die nichtbildgebenden Vertreter gleichermaßen[22, 3].

\subsubsection{Historie}

Startpunkt ist die Brewsterwinkelmikroskopie, die von Dr. D. Hönig in seiner Dissertation [30] entwickelt und anschließend durch die Firmengründung (ehemals $\mathrm{Na}$ nofilm) kommerziell vertrieben wurde. Mit Hilfe dieser Technik war es erstmals möglich, Lipidfilme an der Luft-Wasser-Grenzfläche zu visualisieren und deren physikalisches Verhalten in Kombination mit LB-Isothermen zu untersuchen. Bei dieser 
optischen Methode wird p-polarisiertes Licht verwendet, das am Brewsterwinkel von Wasser in Totalreflexion ins wässrige Medium gebrochen wird. Erst wenn sich eine nanoskalige Schicht im Strahlengang befindet, wird das Licht reflektiert und mittels CCD-Kamera detektiert.

Eine Dekade später - nach der Veröffentlichung von D. Beaglhole [9] über das erste abbildende Ellipsometer - wurde durch eine Erweiterung des Aufbaus des Brewsterwinkelmikroskops um einen Kompensator und Analysator eines der ersten kommerziellen erwerblichen abbildenden nulling Ellipsometer entwickelt. Anfangs wurde das System nur mit einem Laser als Lichtquelle betrieben. Somit war es zunächst auf Anwendungen im Bereich der Einwellenellipsometrie limitiert.

Aufgrund des Fortschritts von Programmierung und Computerleistung im Bereich der Wissenschaft wurde das System zu einem spektroskopisch betriebenen abbildenden Ellipsometer erweitert. Dadurch wurde dem generellen Trend der Anwendung von spektroskopischer Ellipsometrie (Abbildung $3.7^{20}$ ) gefolgt.

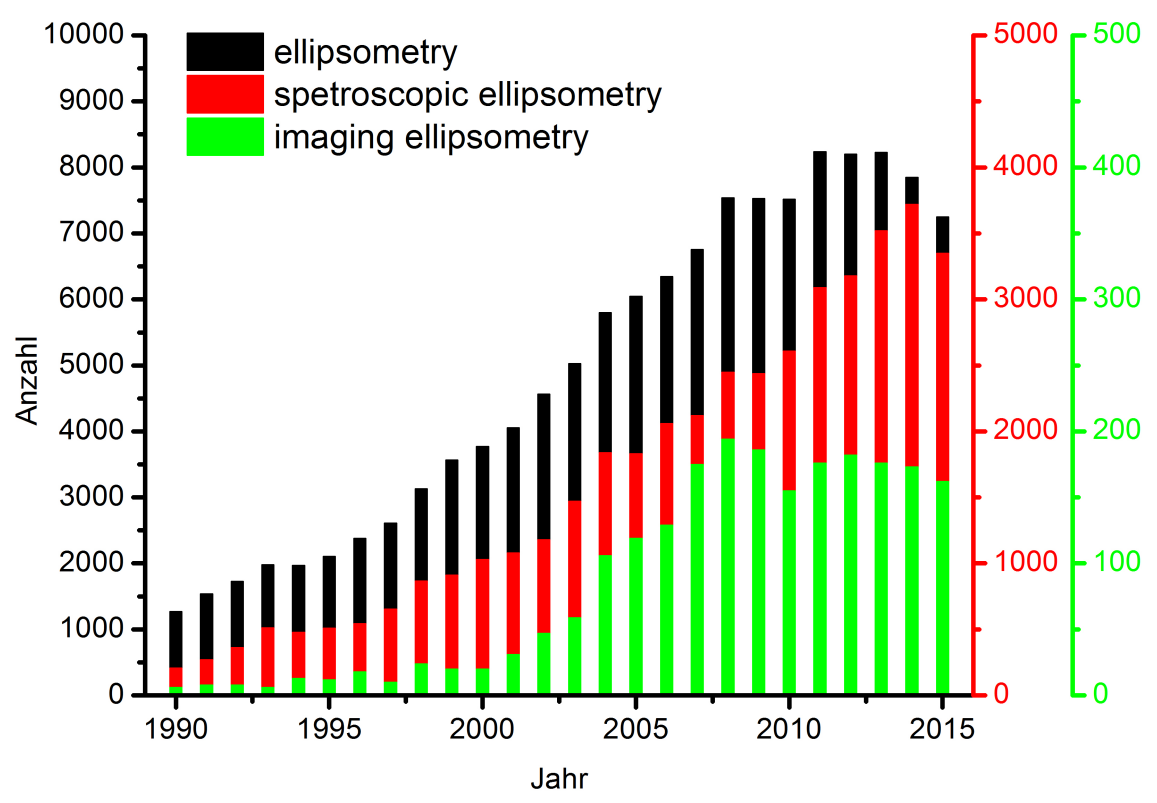

Abbildung 3.7 Anzahl von Veröffentlichungen im Zeitraum von 1990-2015.

\subsubsection{Ellipsometric Platform (EP)}

\section{Aufbau}

In Abbildung 3.8 wird eine EP4-SE gezeigt.

Auf der linken Seite befindet sich der breitbandigen Dioden-Laser $((1), \lambda=658 \mathrm{~nm}$, $\Delta \lambda_{\mathrm{sp}}=1 \mathrm{~nm}, \mathrm{P}=50 \mathrm{~mW}$, PIC) vor dem sich ein Abschwächer befindet (2). Zwischen dem Laser und der fasereingekoppelten spektroskopischen Lichtquelle (Kapitel 4.2.1) wird über einen Klappspiegel (3) gewechselt. Als optische Komponenten

\footnotetext{
${ }^{20}$ Diese Darstellung basiert auf der Stichwortsuche bei google scholar
} 


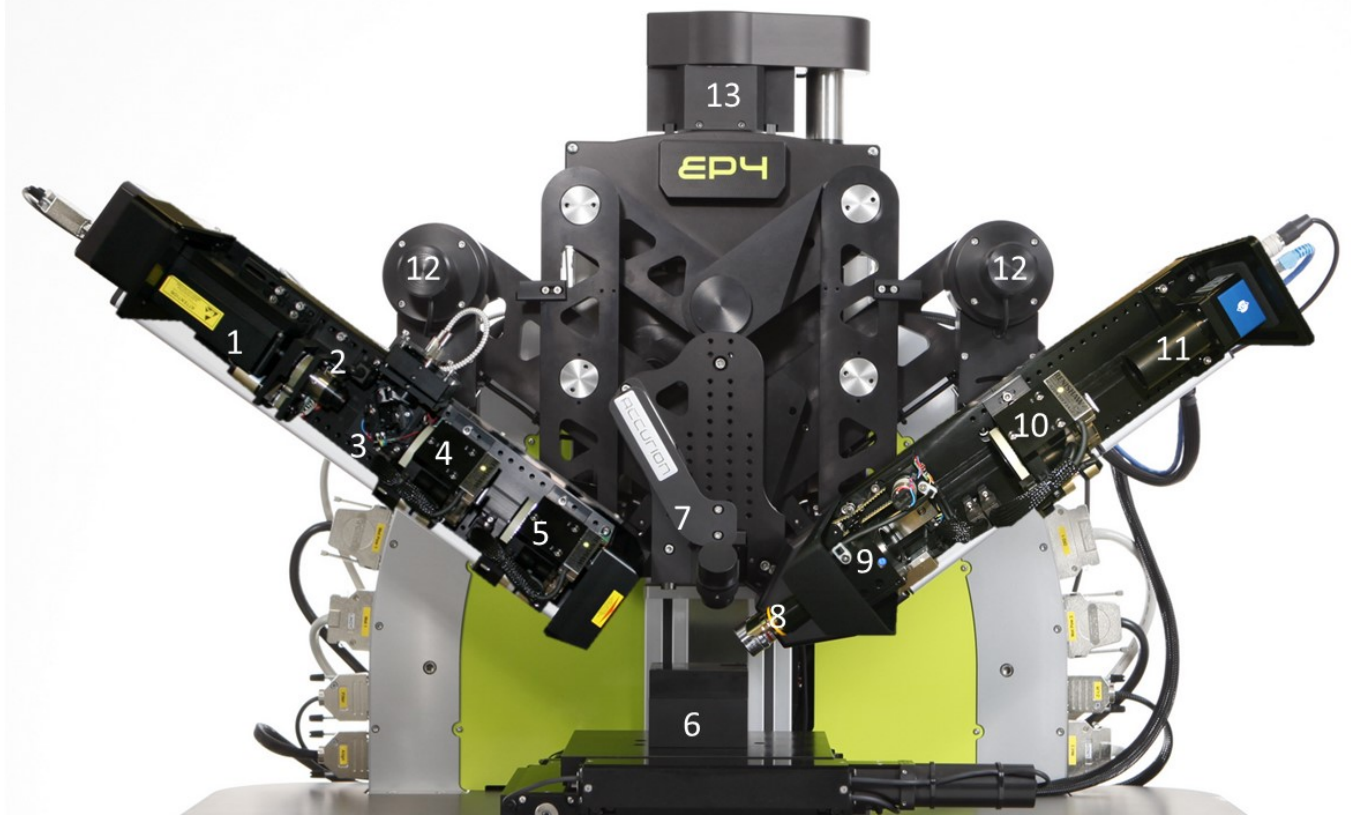

Abbildung 3.8 Aufbau einer EP4-SE.

(Polarisator (4) und Analysator (10)) werden Glam-Thompson Polarisationsprism verwendet, die über einen dekodierten Drehversteller, mit einer Präzision von $0.001^{\circ}$, rotiert werden. Als Kompensator (5) wird eine achromtische Verzögerungsplatte, bestehend aus Quarz und $\mathrm{MgF}_{2}$, benutzt. Beim Probentisch handelt es sich um einen automatisch steuerbaren xy-Tisch (6) mit einem Verfahrweg von $100 \mathrm{~mm}$ in beide Richtungen. Das Alignment, das sich beim Vorgängermodell (EP3) noch im xy-Tisch befand, wurde an die Aufhängung des Gerätes gesetzt, um nicht die Probe sondern das komplette Gerät auszurichten. Mit dem neuen Alignmentsensor (7) kann neben dem Alignment zusätzlich die z-Höhe automatisch eingestellt werden. Analog zu dem schematischen Aufbau eines Ellipsometers in der PCSA-Konfiguration wird beim bildgebenden System der Firma Accurion ein Objektiv (8) zwischen Probe und Analysator eingeführt. Dabei bestimmt das Objektiv die Größe des Sichtfeldes (field of view FOV) der Kamera ((11), $1392 \times 1040$ pixel, 12 bits) und die maximal laterale Auflösung. In Tabelle 3.1 ist die laterale Auflösung und Sichtfeld der Objektive zusammengefasst. Der Focus-Scanner (9) verfährt je nach Vergößerung das Objektiv um komplett gescannte Bilder zu erhalten. Die beiden Arme sind an einem motorisierten Goniometer (12) befestigt, womit Winkel im Bereich von $38^{\circ}-90^{\circ} \mathrm{mit}$ einer Genauigkeit von $0.001^{\circ}$ eingestellt werden können.

Das Messprinzip des „Nullings" bleibt bestehen. Das Signal wird von der CCDKamera detektiert und mittels einer Software digitalisiert. Das Gerät wird anhand des Brewsterwinkels von Wasser kalibriert, wobei darüber hinaus die optischen Achsen der optischen Komponenten anhand dieser Grenzfläche iterativ eingestellt werden. ${ }^{21}$

\section{Regions of interests}

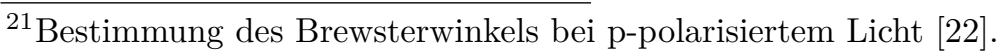




\begin{tabular}{|c|c|c|}
\hline Vergrößerung & laterale Auflösung & FOV $_{\mathrm{x}}$ \\
\hline \hline $2 \mathrm{x}$ & $8 \mu \mathrm{m}$ & $2 \mathrm{~mm}$ \\
\hline $5 \mathrm{x}$ & $3 \mu \mathrm{m}$ & $900 \mu \mathrm{m}$ \\
\hline $10 \mathrm{x}$ & $2 \mu \mathrm{m}$ & $400 \mu \mathrm{m}$ \\
\hline $20 \mathrm{x}$ & $1 \mu \mathrm{m}$ & $200 \mu \mathrm{m}$ \\
\hline $50 \mathrm{x}$ & $0.6 \mu \mathrm{m}$ & $70 \mu \mathrm{m}$ \\
\hline
\end{tabular}

Tabelle 3.1 Laterale Aufösung und Größe des Sichtfgeldes in $\mathrm{x}$-Richtung der eingesetzten Objektive.

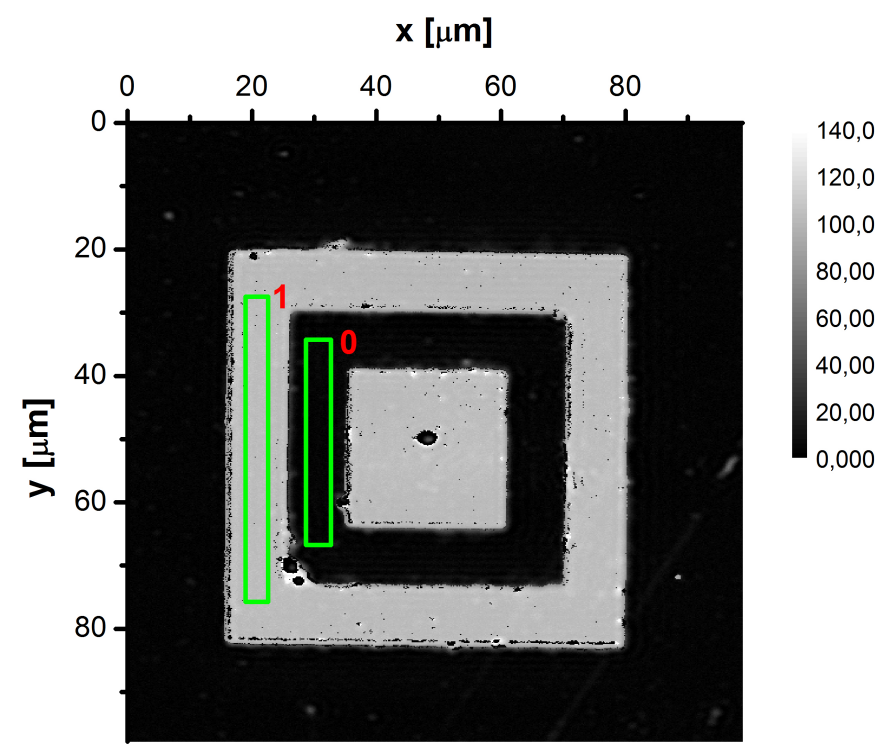

Abbildung 3.9 Region of Interest in einem gescannten Image einer mikrostrukturiten $\mathrm{SiO} 2-\mathrm{Schicht}$ auf $\mathrm{Si}(100)$.

Das Messkonzept des abbildenden Ellipsometers besteht darin, geeignete Bereiche auf der Probe auszuwählen und diese mittels sogenannter Regions of Interests (ROIs) zu markieren (Abbildung 3.9). Die ellipsometrischen Parameter Delta und Psi werden nur in den gewünschten Bereichen von der CCD-Kamera detektiert. Im Vergleich dazu wird bei herkömmlichen Ellipsometern das gesamte reflektierte Licht detektiert. Zwar lässt sich durch geeignete Fokussierung der Lichtquelle - sogenannte Microspots - der reflektierte Bereich auf der Probe eingrenzen, dabei können allerdings keine Spotdurchmesser unter $30 \mu \mathrm{m}$ realisiert werden. Zusätzlich können mittels abbildender Ellipsometrie die Signale mehrerer ROIs gleichzeitig aufgezeichnet werden. Die Bildgebung des Ellipsometers ermöglicht es, mikrostrukturelle Proben zu vermessen oder gewünschte Strukturen zu detektieren und im Live-Bild sichtbar zu machen.

\section{Grabs und Scans}



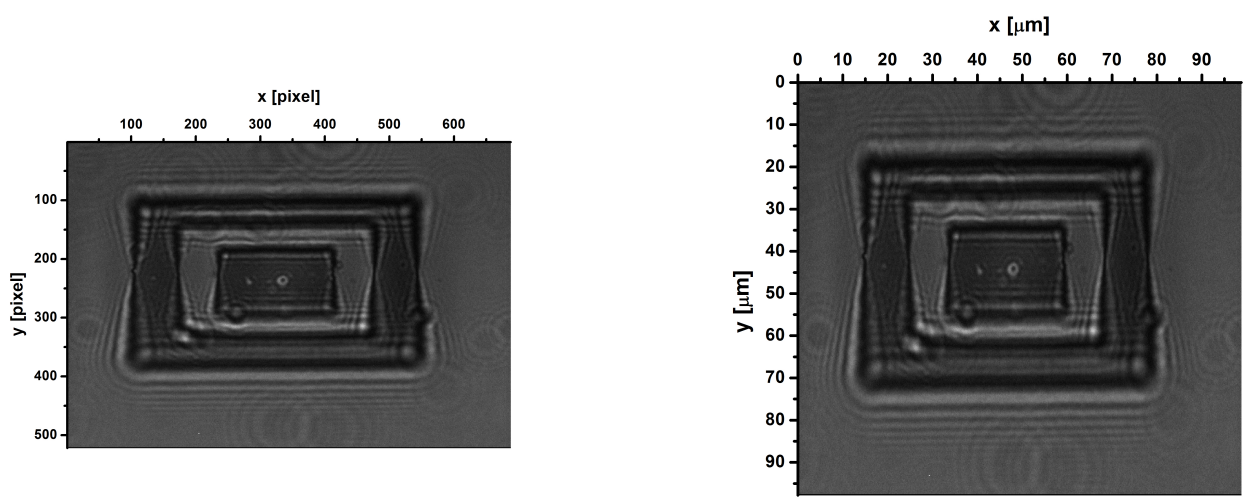

Abbildung 3.10 Bildkorrektur eines Grabs einer strukturierten $\mathrm{SiO} 2$ Schicht auf $\mathrm{Si}(100)$ aufgenommen bei einem AOI von $50^{\circ}$ und Verwendung des 50x Objektivs.

Bei dem abbildenden Ellipsometer sieht der Anwender in der Bedienungssoftware ein Live-Bild der Probe, das von der Kamera generiert wird. Bereits im Live-Bild wird eine Auto-Korrektur durchgeführt, denn aufgrund des gewinkelten Aufbaus verzerrt sich die Darstellung der Probe in Richtung der Einfallsebene. In Abhängigkeit von der Größe des Betrachtungswinkels (angle of view, AOV), entspricht die Summe der Pixel in Y-Richtung einer Länge von:

$$
l_{\mathrm{y}}=l_{\mathrm{FOV}, \mathrm{x}} \cdot \frac{1}{\cos (A O V)}
$$

Dazu wurden bei der Kalibrierung des Geräts das FOV (in $\mu m$ ) für jedes Objektiv in X-Richtung bestimmt. Der Betrachtungswinkel $A O V$ (in ${ }^{\circ}$ ) wird vom Goniometer angegeben, wobei die Software die entsprechende Korrektur des Bildes automatisch durchführt. Vor allem beim Exportieren der Daten (ASCII etc.) ist es wichtig, zu wissen, dass es sich um eine Streckung der Länge pro Pixel handelt und nicht um eine Interpolation der Werte.

In Abbildung 3.10 sieht man den direkten Vergleich zwischen dem unkorrigierten und korrigierten Live-Bild ( $50 x$ Objektiv, $\mathrm{AOI}=50^{\circ}$ ) einer mikrostruktierten $\mathrm{SiO}_{2}{ }^{-}$ Schicht $(\mathrm{d}=100 \mathrm{~nm})$ in quadratischer Form auf Silizium.

Aufgrund des schrägen Betrachtungswinkels sieht man zusätzlich im Live-Bild (später auch als grab bezeichnet) nur eine horizontale Linie scharf. Dieser Effekt wird auch als Scheimpflug bezeichnet. Nur bei der scharfen Linie im Bild sind die Bedingungen zwischen Objekt, Objektiv und Bildebene entsprechend der Brennweite des Objektivs erfüllt.

Um ein komplett scharfes Kontrast-Bild zu generieren wird das Objektiv mit Hilfe des sogenannten Focus-Scanners verschoben und mehrere einzelne Grabs werden aufgezeichnet. Das komplett fokussierte Bild (scan) wird aus den einzelnen scharfen Bereichen der Grabs zusammengesetzt (Abbildung 3.11). 
Grabs

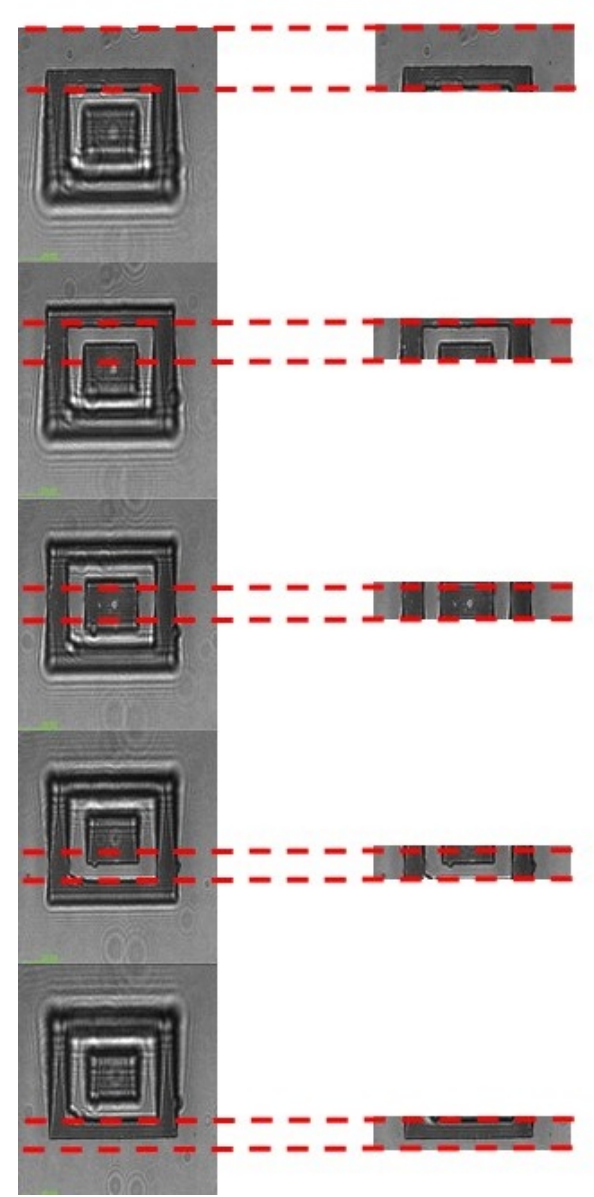

Scan

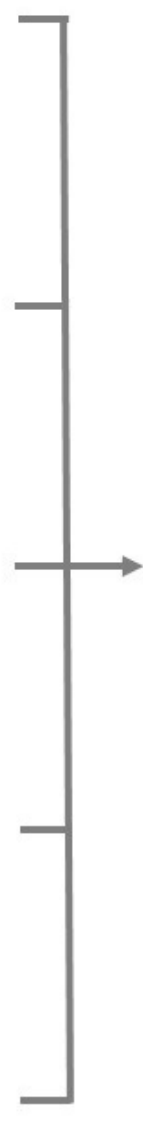

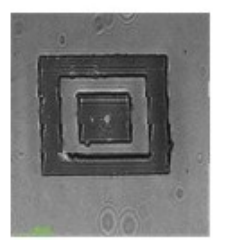

Abbildung 3.11 Scanning Prozess

Dabei ist zu beachten, dass der Verfahrweg des Fokus-Scanners vom jeweiligen Objektiv abhängig ist und im Vorfeld in der Software vom Anwender ausgewählt werden muss. Zusätzlich kompensiert das Programm die wellenabhängige Brennweite des Objektivs, so dass die Fokuslinie, die manuell gesetzt werden muss, auf konstanter Y-Position bleibt. 


\section{Mapping}
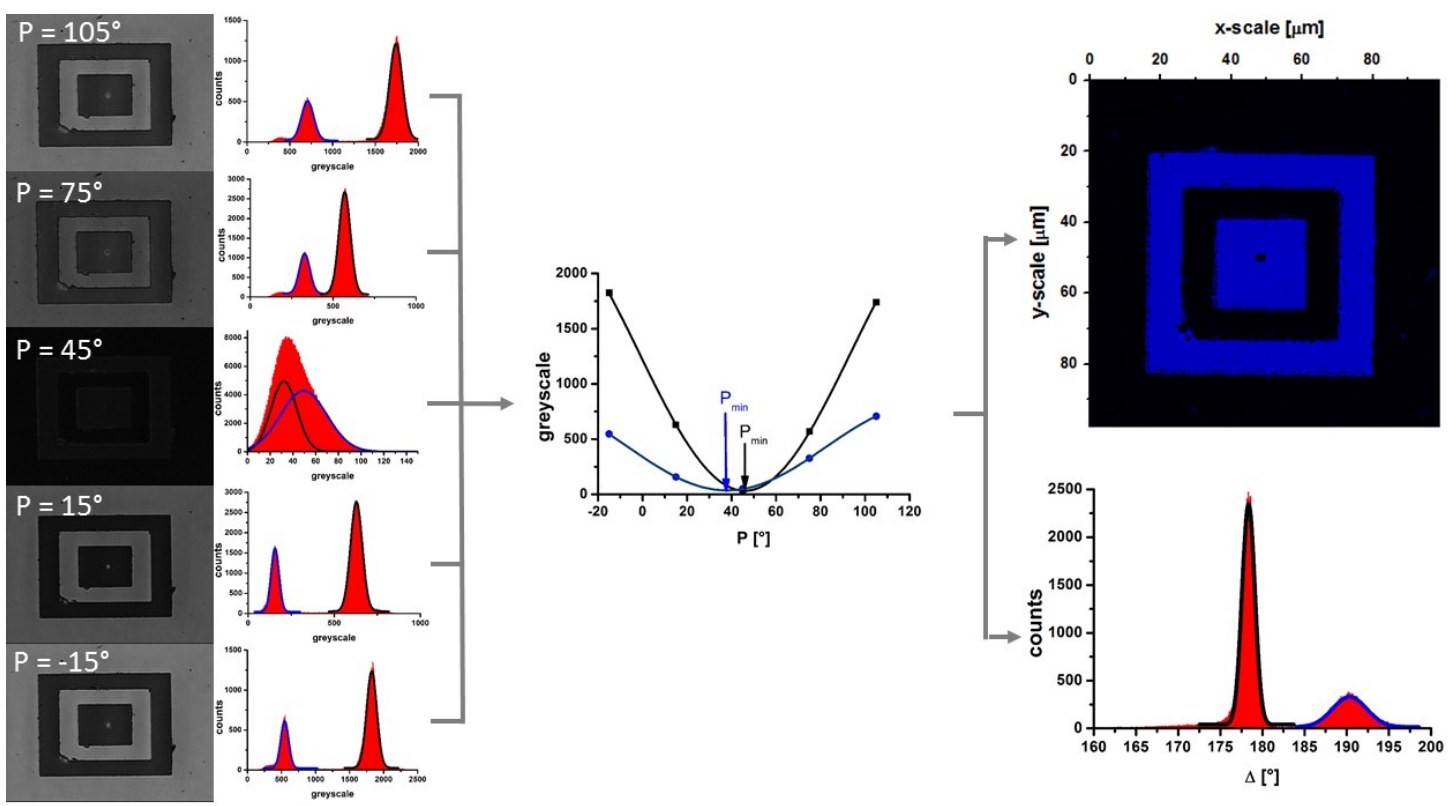

Abbildung 3.12 Mapping von Delta einer mikrostruktuirerten SiO2 Schicht auf $\mathrm{Si}(100)$

Neben dem Konzept der ROIs ist im besonderen die Option des Mappings einer der wichtigsten Aspekte der abbildenden Ellipsometrie. Hierbei wird zu jedem Pixel der Abbildung von der Probe ein Delta-Psi-Paar berechnet und als 2D-Array ( map) dargestellt. Prinzipiell lässt sich der Messvorgang als Kombination von „Nulling“ und "Scanning" betrachten. Der Prozess des Mappings wird anhand einer Delta-map detailierter in Abbildung 3.12 gezeigt. Klassisch wie beim „Nulling" wird der Polarisator entsprechend der Vorgaben (ranges) des Anwenders aus der „null“-Position gefahren, während der Analysator seine Position beibehält. Es wird schrittweise bei den jeweiligen Polarisatorstellungen (bei statischem P) ein scan durchgeführt (Abbildung 3.12, links). Anschließend wird anhand der Graustufen der scans, eine Delta-Map - Minimum der Intensität pro Pixel - berechnet. Die graphische Darstellung der Intensität in Abhängigkeit von der Stellung des Polarisators wird im Zentrum der Abbildung 3.12 gezeigt. Die resultierende Deta-Map ist auf der rechten Seite zu sehen mit dem dazugehörigen Histogramm der Delta-Werte. Analog dazu wird das Mapping einer Psi-map bei schrittweiser Analysatorrotation und fester Polarisatorstellung durchgeführt. 


\subsubsection{Software}

\section{Ep3 - Ep4}

Die EP3 wird über das Programm EP3_View vom Anwender bedient. Die scriptbasierte, extern entwickelte Software bietet in Form eines Funktionsbaums folgende Grundfunktionalitäten:

Grab, Scans, Maps (1 Zone), Brewsterwinkelmessung, AOI-Spektren (1-4 Zonen), Lambda-Spektren (1-4 Zonen), VASE-Spektren (1-4 Zonen) sowie kinetische Messungen (1 Zone). Die generierten Daten werden im Logeditor erfasst und gespeichert. Alle bildbasierenden Daten (Scans, Maps) werden im Imageviewer angezeigt, bearbeitet und verwaltet. Die Datenanalyse wird im EP4-Model durchgeführt. Zu Beginn der Arbeit stand zunächst die erste Version des EP4-Models zur Verfügung, die inhouse entwickelt und zusammen mit der neuen Gerätegeneration EP4 weiterentwickelt wurde.

Bei der neuen Gerätegeneration Ep4 werden im Gegensatz zum Ep3_View die Gerätesteuerung (Control) und Datenverarbeitung (DataStudio) separiert. Für den Anwender stehen neue zusätzliche Optionen wie Mapping (4 Zonen) und ungescannte Maps zur Verfügung. Alle Daten, sowohl numerische als auch 2D-Arrays (Scans, Grabs, Maps), werden automatisch ins Datastudio gesendet und dort verwaltet, bearbeitet und gespeichert.

\subsubsection{Datenanalyse}

Bei der Ellipsometrie handelt es sich um eine indirekte Methode um messrelevante Werte (n,k,d) mittels geeigneter Modellierung des Probensystems (Schichtaufbau, Dispersion, Medium) zu bestimmen. Abbildung 3.13 visualisiert das systematische Vorgehen bei der Auswertung experimenteller Daten.

Im Ep4_Model wird anhand der optischen Parameter der Materialien und des Probenaufbaus ein Delta- und Psi-Spektrum simuliert, das bei exakter Beschreibung des Systems bestenfalls deckungsgleich mit den detektierten Delta-Psi-Wertepaaren ist und somit das Reflexionsverhalten der Probe widerspiegelt. Die gemessenen Daten werden als Punkte und das simulierte Spektrum wird in linearer Form dargestellt. Das Reflexionsverhalten der Materialien wird mittels geeigneter optischer Funktionsmodelle (siehe Kapitel 2.6.4) oder unter Einbeziehung bekannter optischer Konstanten aus Datenbanken parametrisiert. 


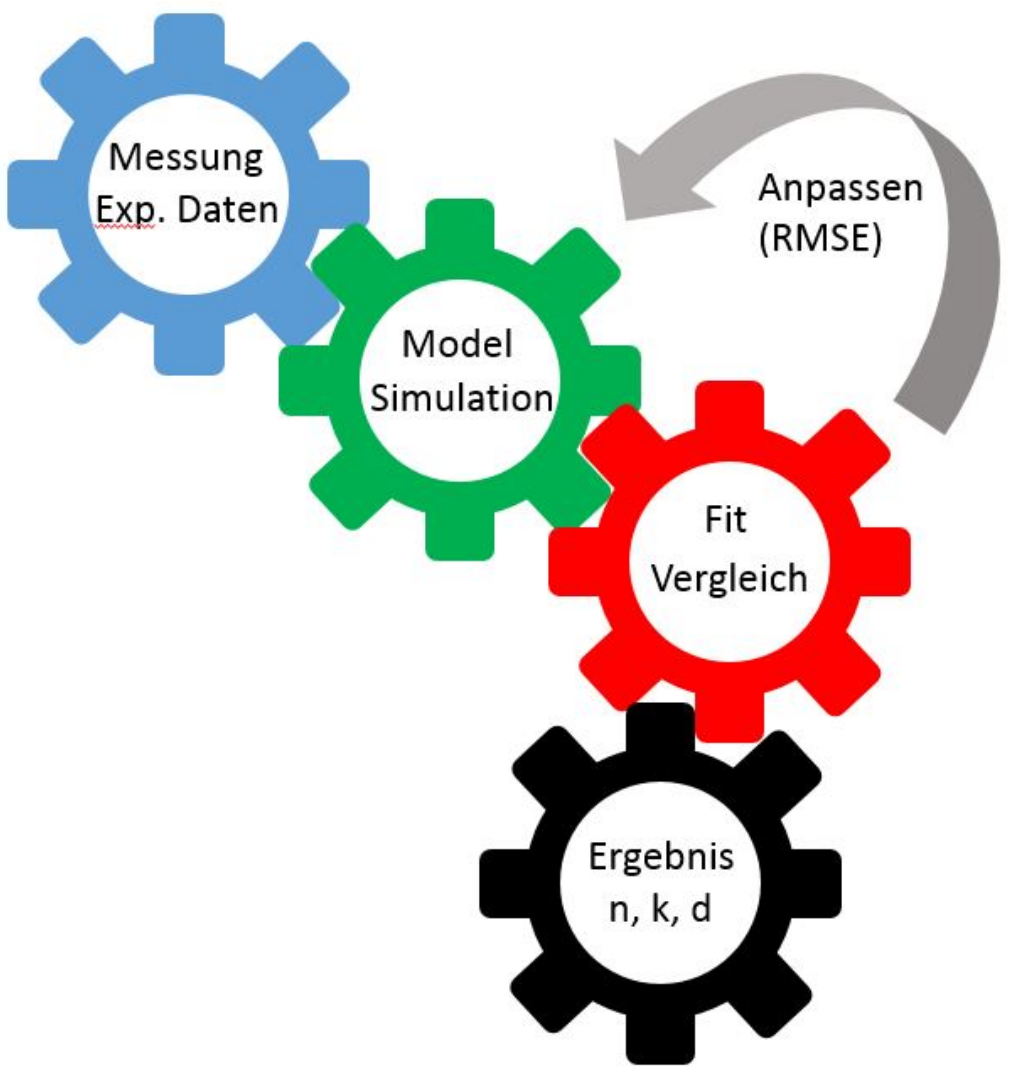

Abbildung 3.13 Schematischer Verlauf der Datenanalyse bei der Ellipsometrie.

Nach entsprechender Zuordnung im Schichtsystem (Medium, Schicht, Substrat) werden die Messparameter (Parameter des optischen Models, Schichtdicke) mittels Fitting Prozess nach Levenberg-Marquvart-Methode sukzessive angepasst, bis die Abweichungen der simulierten Daten, verglichen mit den experimentellen Werten, minimal sind[24]. Als Qualitätsmerkmal, in welchem Maß das optische Model dem realen System der Probe entspricht, nutzt man die Wurzel aus der mittleren quadratischen Abweichung (root mean square error (RMSE)).

$$
R M S E=\frac{1}{2 N-M} \sum_{i=1}^{N}\left[\left(\frac{\Psi_{i}^{\bmod }-\Psi_{i}^{\exp }}{\sigma_{\Psi, i}^{\exp }}\right)^{2}+\left(\frac{\Delta_{i}^{\bmod }-\Delta_{i}^{\text {exp }}}{\sigma_{\Delta, i}^{\exp }}\right)^{2}\right]
$$

N repräsentiert die Anzahl zusammengehöriger Delta-Psi-Paare, M die Anzahl der Variablen und $\sigma$ die Standardabweichung der experimentellen Daten. Die abgekürzten Indizes mod und exp stehen für die modellierten und experimentell bestimmten Werte. Welchen Wert der RMSE generell besitzen soll, lässt sich nicht pauschalisieren. Dieser hängt von der Komplexität des Modells und sonstigen Eigenschaften der Probe ab. 


\subsection{Zusätzliche Messmethoden}

\subsubsection{ATR-FTIR-Spektroskopie}

Um zusätzliche Informationen über die Dünnschichtproben zu generieren, wurden neben den ellipsometrischen Messungen ebenfalls IR-Messungen mittels ATR-Kristall durchgeführt. Die Infrarot-Spektren in dieser Arbeit wurden in Reflexion unter Vewendung des IR-Mikroskops Hyperion3000 (Bruker) im ATR-Modus (GermaniumKristall, 20x Objektiv) aufgenommen. Für einen tieferen Einblick hinsichtlich InfrarotSpektroskopie sei auf die einschlägige Literatur "IR Spektroskopie: Eine Einführung“ von H. U. GREMLich [26] verwiesen.

\subsubsection{Infrarot-Spektroskopie}

Analog zur Ellipsometrie ist das Prinzip dieser spektroskopischen Messmethode ebenfalls die Wechselwirkung zwischen elektromagnetischer Strahlung und Materie. Wie in Abbildung 2.10 dargestellt, wird hierbei jedoch Infrarotstrahlung $(\lambda=1 \mu \mathrm{m}-1 \mathrm{~mm})$ verwendet, deren energetischer Gehalt sich im Bereich von Schwingungen von Molekülen befindet. Trifft nun Licht entsprechender Energie auf ein Molekül, so wird die Strahlung absorbiert und das Molekül zum Schwingen angeregt. Dabei sind aber nur Schwingungsmoden IR-aktiv, bei denen sich das Dipolmoment des Moleküls ändert.

Das Prinzip der Absorption lässt sich vereinfacht für ein zweiatomiges Molekül anhand des Modells eines harmonischen Oszillators (Gleichung 3.5) beschreiben:

$$
\nu=\frac{1}{2 \pi} \cdot \sqrt{\frac{k_{\text {Bindung }}}{\mu_{\text {Molekül }}}}
$$

In diesem Modell wird das Energiepotential der Bindung durch eine Parabel mit äquidistanten Energieniveaus beschrieben. Reale Effekte wie Abstoßung der Atome bei geringem Abstand und Brechung der Bindung bei großem Abstand der Atome, werden dabei nicht berücksichtigt, weshalb das Modell nur bei geringen Auslenkungen der Atome gilt.

\subsubsection{Fourier-Transform-IR-Spektroskopie}

Im Gegensatz zu dispersiver IR-Spektroskopie, bei der monochromatische Strahlung genutzt wird, ermöglicht die Fourier-Transform-IR-Spektroskopie, polychromatisches Licht zu nutzen. Dabei wird das Gitter oder Prisma, das die monochromatische Strahlung erzeugt, durch ein Michelson-Interferometer (Abbildung 3.14) ersetzt. 


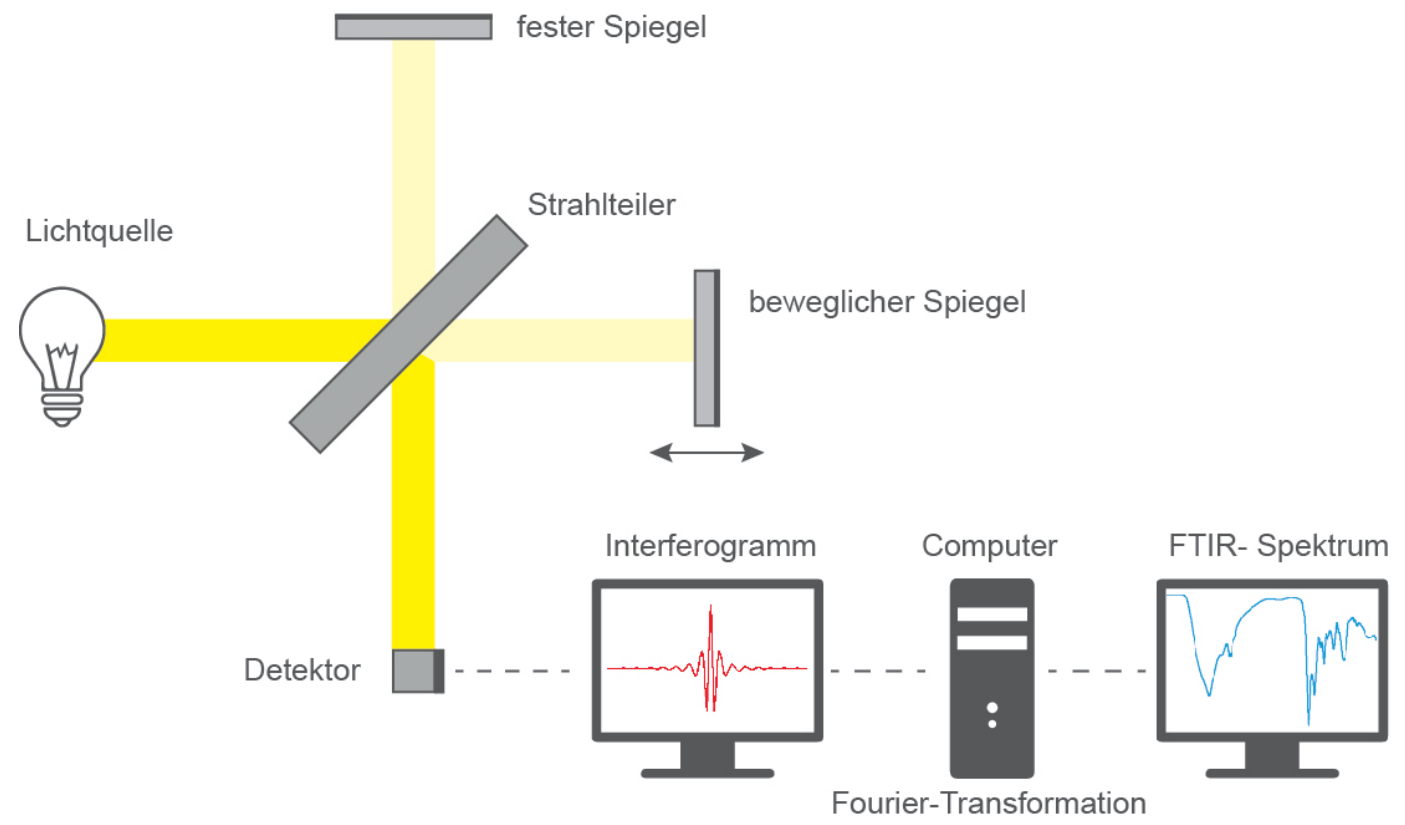

Abbildung $\mathbf{3 . 1 4}$ Schematischer Aufbau eines FTIR-
Spektrometer [26].

Im Wesentlichen besteht ein Michelson-Interferometer aus einem Strahlteiler und zwei Spiegeln, wobei einer der beiden Spiegel beweglich ist. Am Strahlteiler wird die Strahlung in zwei Teilstrahlen gesplittet. Dabei wird der eine Teilstrahl auf den fixierten Spiegel gelenkt und der andere auf den beweglichen Spiegel. Nach Reflexion interferieren die beiden Teilstrahlen am Strahlteiler, treffen auf die Probe und werden schlussendlich detektiert. In Abhängigkeit der Auslenkung des beweglichen Spiegels kommt es zur Interferenz - konstruktiv oder destruktiv - der Teilstrahlen. Somit enthält das Interferogram, in Form des Detektorsignals in Abhängigkeit der Spiegelposition, die spektrale Information der Lichtabsorption an der Probe. Durch eine anschließende Fourier-Transformation wird die Ortsinformation des Spiegels in eine Energie- bzw. Frequenz-Information umgerechnet und man erhält die absolute Intensitätsverteilung über den gesamten spektralen Bereich der Infrarotstrahlung. Neben der erheblichen Messzeitersparnis ${ }^{22}$ zeichnet sich die FTIR-Spektroskopie durch eine besonders hohe Wellenzahlgenauigkeit ${ }^{23}$ und ein gegenüber der klassischen IR-Spektroskopie besseres Signal-Rausch-Verhältnis ${ }^{24}$ aus.

\subsubsection{Attenuated Total Reflection FTIR-Spektroskopie}

Im Jahre 1969 führten die Wissenschaftler Harrick und Fahrenfort die AttenuatedTotal-Reflection-Spektroskopie (abgeschwächte Totalreflexion) ein. Aufgrund der noninvasiven Arbeitsweise, des simplen Handlings der Probe und dem geringen apparativen Aufwand wird diese Technik häufig zur Charakterisierung von Oberflächen auf

\footnotetext{
${ }^{22}$ Fellget Vorteil

${ }^{23}$ Connes Vorteil

${ }^{24}$ Jaquinot-Vorteil
} 
molekularer Ebene eingesetzt [29]. Wie der Name vermuten lässt, findet bei dieser Form der IR-Spektroskopie ein Übergang zwischen einem optisch dichteren Medium (Internal Reflection Element, IRE) zu einem optisch weniger dichten Medium (Probe) statt. Ab dem kritischen Winkel (siehe Kapitel 2.3) kommt es an der Grenzfläche zur Interferenz zwischen einfallender und reflektierter Strahlung und es kommt zur Ausbildung einer stehenden Welle (evaneszentes Feld), die sich auch außerhalb des IREs ausbreitet (Abbildung 3.15).

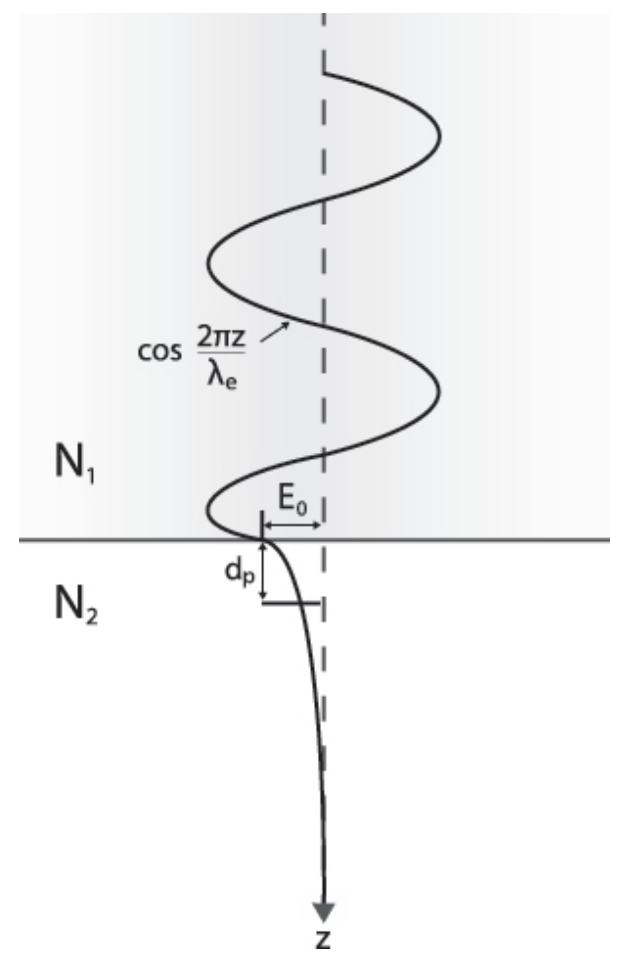

Abbildung 3.15 Schema des evaneszenten Feldes.

Dabei fällt die Intensität des evaneszenten Feldes mit zunehmendem Abstand z von der Grenzfläche exponentiell:

$$
E=E_{0} \cdot \exp \left(-\frac{z}{d_{\mathrm{p}}}\right)
$$

mit $[29]$

$$
d_{\mathrm{p}}=\frac{\frac{\lambda}{n_{1}}}{2 \pi \sqrt{\sin ^{2} \theta-\left(\frac{n_{3}}{n_{1}}\right)^{2}}}
$$

Die Eindringtiefe $d_{\mathrm{p}}$ (depth of penetration) in das Substrat ist laut Definition jener Abstand, bei dem die Amplitude des evaneszenten Feldes ca. 37 \% beträgt. 


\subsubsection{Rasterkraftmikroskopie}

Die Rasterkraftmikroskopie (atomic force microscopy) ist im Vergleich zur Ellipsometrie eine relativ neue Messmethode ${ }^{25}$ um strukturelle und elastische Eigenschaften von Oberflächenmaterialien zu charakterisieren. Im Gegensatz zur Ellipsometrie handelt es sich um eine direkte Messmethode, bei der sich eine atomare topographische Abbildung der Oberfläche ohne Modellierung ergibt. Die Aufnahmen in dieser Arbeit wurden mit dem Nanite B (Nanosurf) gemacht und nur bei relevanten Probensystemen genutzt.

\subsubsection{Messprinzip und Aufbau}

Prinzipiell besteht ein Rasterkraftmikroskop aus einem Cantilever, einem Laser und einem optischen Detektionssystem (siehe Abbildung 3.16).

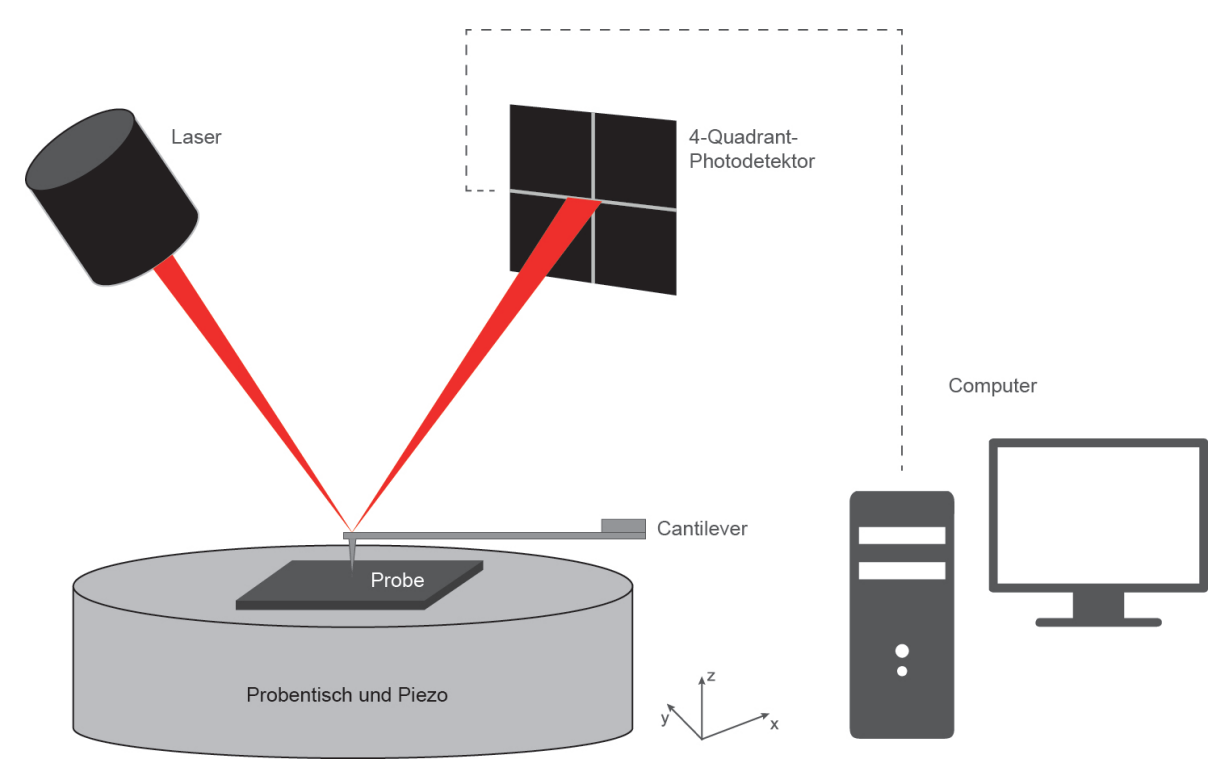

Abbildung 3.16 Schematischer Aufbau eines Rasterkraftmikroskops.

Die Eigenschaften des Cantilevers, seine Resonanzfrequenz $c_{\text {Cantilever }}$ und Federkonstante $f_{\text {Cantilever, }}$ zählen zu den wichtigen messtechnischen Parametern des AFMs.

$$
\begin{aligned}
& c_{\text {Cantilever }}=E_{\text {Modul }} \cdot d^{3} \frac{w}{4 L^{3}} \\
& f_{\text {Cantilever }}=0,162 \sqrt{\frac{E_{\text {Modul }}}{\rho}} \cdot \frac{d}{l^{2}}
\end{aligned}
$$

\footnotetext{
${ }^{25} 1986$ von G. Binning et al.
} 
Um eine maximale laterale Auflösung zu erhalten, sollte im Idealfall der Cantilever eine scharfe Spitze mit einem kleinen Krümmungsradius und einem kleinen Öffnungswinkel besitzen. Beim AFM existieren drei verschiedene Messmodi: „contact"-, „non-contact"- und „tapping“-Modus. In dieser Arbeit wurde nur im erstgenannten Modus gearbeitet, weshalb die anderen beiden Modi lediglich kurze Erwähnung finden: Beim „non-contact“-Modus wird der Cantilever ohne Berührung über die Oberfläche der Probe gefahren. Dabei werden langreichende Wechselwirkungen ${ }^{26}$ zwischen der Oberfläche und der Cantileverspitze ortsaufgelöst gemessen und topographisch festgehalten. Im Gegensatz dazu steht im „contact“-Modus die Spitze des Cantilevers in direktem Kontakt mit der Probenoberfläche und wird entsprechend eines Abtastmusters über die Probe verfahren. Der „tapping"-Modus kann als Hybrid der beiden Messmodi aufgefasst werden. Der Cantilever wird in Schwingung versetzt und ist kurzzeitig in Kontakt mit der Oberfläche. Die Resonanzfrequenz des Cantilevers bestimmt den zu nutzenden Messmodus des AFMs ${ }^{27}$.

Beim verwendeten „contact“-Modus werden vom AFM die interatomaren Wechselwirkungen sowie vertikale und laterale Reibungskräfte gemessen. Diese entstehen zwischen Probe und Cantileverspitze, die sich wiederum im repulsiven Kontakt zur Oberfläche befindet. Beim Messvorgang selbst wird die z-Position des Cantilevers durch eine Rückkopplungsschleife des Piezo-Elements so gesteuert, dass die Auslenkung des Cantilevers und somit auch der Abstand zwischen Probe und Cantileverspitze an allen Punkten näherungsweise konstant ist. Die Oberfläche wird nun in der $\mathrm{x}$-y-Ebene abgerastert, wobei es aufgrund von Unebenheiten der Oberfläche zur Auslenkung oder lateralen Verbiegung des Cantilevers kommt. Die Auslenkung beziehungsweise Verbiegung wird durch einen auf das Ende des Cantilevers fokussierten Laser, dessen Strahl auf einen 4-Quadranten-Photodetektor reflektiert wird, in eine Spannungsdifferenz zwischen den Photodioden transformiert:

$$
\delta=S_{\mathrm{D}} \cdot \Delta U
$$

Somit besteht über der Deflection Sensitivity $S_{\mathrm{D}^{-}}$als Umrechnungsfaktor ${ }^{28}$ - ein linearer Zusammenhang zwischen der Auslenkung beziehungsweise Torsion des Cantilevers und der Spannungsdifferenz des Photodetektors. Unter Verwendung des Hookschen Gesetzes ergibt sich für die Kraft F:

$$
F=c_{\text {Cantilever }} \cdot \delta
$$

Der Cantilever $\left(c_{\text {Cantilever }}=10 \mathrm{kHz}\right)$ besteht beim Nanite $B$ aus Silizium mit einer integrierten Si-Spitze in pyramidaler Form (konischer Winkel von 20-25 ) mit einem Radius von $10 \mathrm{~nm}$. Das AFM wird im „contact"-Modus betrieben.

\footnotetext{
${ }^{26}$ van-der-Waals-, elektrostatische oder magnetische Wechselwirkungen

${ }^{27} f_{\text {contact }}=10-15 \mathrm{kHz}, f_{\text {non-contact }}=120-290 \mathrm{kHz}, f_{\text {tapping }}=300-400 \mathrm{kHz}$

${ }^{28}$ Bestimmung durch Kalibrierung
} 


\subsubsection{Kontaktwinkel}

Kontaktwinkelmessungen sind eine sehr verbreitete und sensitive Technik zur Charaketeriserung der chemischen oder physikalischen Eigenschaften von Oberflächen [11]. Sie runden neben der ATR-FTIR-Spektroskopie und Ellipsometrie das Charakterisierungsprofil der Proben ab. Das Benetzungsverhalten verschiedener Materialien spielt bei einigen Prozessen eine entscheidende Rolle, wie beispielsweise bei Druckvorgängen oder sich selbst reinigenden Oberflächen. In diesem Unterkapitel erfolgt zunächst ein kurzer Blick auf den theoretischen Hintergrund, wobei auf die grundlegende Modellbeschreibung nach Young eingegangen wird. Darauf aufbauend wird die Low Bond Axsymmetric Drop Shape Analysis (LBADSA) erklärt und an einem Standardsystem erläutert.

\subsubsection{Messprinzip und Aufbau}

Definitionsgemäß beschreibt der Kontaktwinkel $\theta$ die physikalische Beziehung zwischen der Oberflächenspannung der Flüssigkeit $\gamma_{\mathrm{lv}}$ und dem Festkörper $\gamma_{\mathrm{sv}}$, sowie der Grenzflächenspannung $\gamma_{\mathrm{sl}}$ zwischen den beiden Phasen:

$$
\cos \theta=\frac{\gamma_{\mathrm{sv}}-\gamma_{\mathrm{sl}}}{\gamma_{\mathrm{lv}}}
$$

Der Kontaktwinkel, der von den physikalischen und chemischen Eigenschaften der Oberfläche sowie der genutzten Flüssigkeit abhängt, ist ein Charakteristikum zur Bestimmung der Oberflächenspannung einer Probe. Wird als Flüssigkeit Wasser verwendet spricht man in diesem Zusammenhang auch von hydrophilen (Kontaktwinkel $<90^{\circ}$ ) bzw. hydrophoben (Kontaktwinkel $>90^{\circ}$ ) Eigenschaften des Materials. Dabei ist besonders wichtig, darauf zu achten, dass Flüssigkeiten verwendet werden, die dem Festkörper gegenüber inert sind und in reiner Form genutzt werden. Dadurch wird gewährleistet, die Messungen untereinander adäquat vergleichen zu können. Eine ideale Probe sollte homogen, kontaminationsfrei und eben sein. Auch physikalische Parameter wie Temperatur, Druck und Lufteuchtigkeit sollten konstant gehalten werden.

Um den Kontaktwinkel geometrisch zu bestimmen, wird nach der Positionierung des - in Form eines Bildes festgehaltenen - Flüssigkeits-Tropfens eine Tangente als „drei-Phasen-Kontaktlinie“ angelegt und der Winkel zur Oberfläche bestimmt (Abbildung 3.17).

Dazu wird das System mittels einer Lichtquelle beleuchtet und durch eine Kamera, die im $90^{\circ}$-Winkel zum Einfallslot der Oberfläche positioniert ist, wird der vom Tropfen erzeugte Schatten detektiert. In dieser Arbeit wurde die Low Bond Axisymmetric Drope Shape Analysis LBDSA Methode angewendet, bei der es sich um eine statische Messung des Kontaktwinkels handelt. Hierbei wird die Krümmung des Tropfens nach folgender Formel beschrieben [51]:

$$
\left(\frac{1}{R_{1}}+\frac{1}{R_{2}}\right)=c \cdot h+\frac{2}{b}
$$


Dabei repräsentieren $R_{1}$ und $R_{2}$ die Krümmung des Tropfens, $c$ steht für die Kapillarkonstante der Flüssigkeit, $h$ für die Höhe des Tropfens im Bezug auf die Probenoberfläche und $b$ für den Radius der Krümmung am Höhepunkt.

Konkret wurde praktisch wie folgt vorgegangen: Der Kontaktwinkel der Proben wurden mit dem abbildenden Ellipsometer bestimmt. Dazu wurde das 5x-Objektiv und als Lichtquelle der Monochromator $(\lambda=500 \mathrm{~nm})$ genutzt. Das System wurde auf einen AOI von $90^{\circ}$ gefahren und der Fokus des Objektivs scharf gestellt. Auf die Oberfläche wurde manuell mittels einer Eppendorf-Pipette ein Wassertropfen (Reinstwasser, $\mathrm{V}=1 \mu l$ ) gegeben und mit dem abbildenden Ellipsometer videoanalytisch verfolgt und eventuell nachfokssuiert. Anhand der Aufnahmen wurde ein geeignetes Bild ausgesucht, auf dessen Grundlage später mittels Software (Image J) unter Verwendung des plug-in (drop_analysis) der Kontaktwinkel bestimmt wurde.

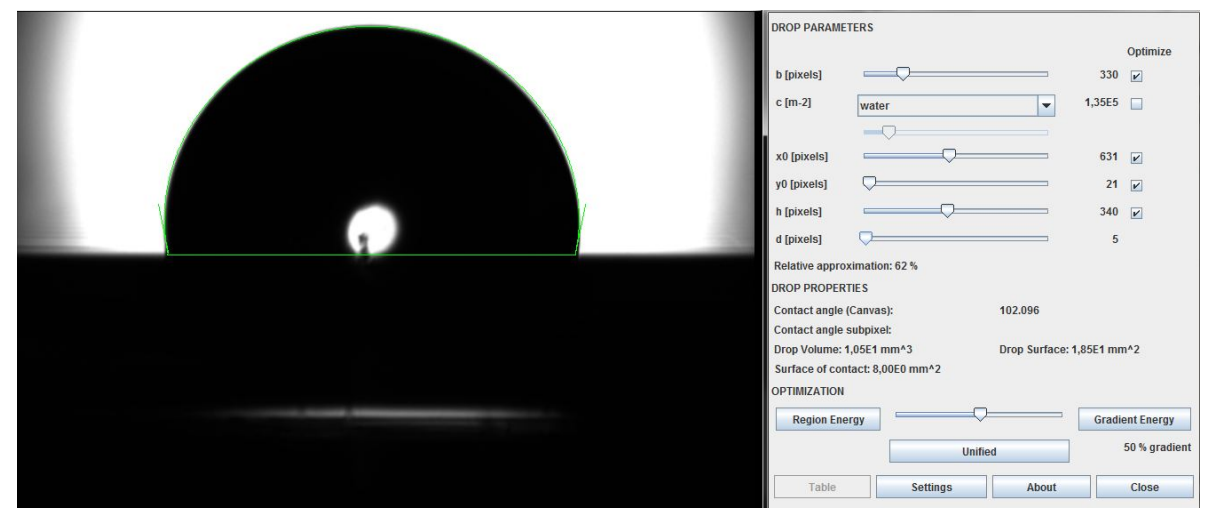

Abbildung 3.17 Bestimmung des Kontaktwinkels von Reinstwasser an $\mathrm{Al} 2 \mathrm{O} 3$ Oberfläche mittels dropshape_analysis (ImageJ)

Die Messungen wurden an fünf verschieden Stellen wiederholt, wobei die Werte statistisch gemittelt wurden. 


\section{Ergebnisse}

Dieses Kapitel gibt einen Überblick darüber, welche neuartigen Möglichkeiten die jeweilige Verbesserung beziehungsweise Erweiterung des Geräts (Hardware und Software) mit sich bringt. Dabei werden die Ergebnisse in vier Kategorien, die den Unterkapiteln entsprechen, zusammengefasst.

\subsection{Imaging}

Wie in Kapitel 3.1.3 beschrieben, ist eine der wichtigsten Möglichkeiten, die das abbildende Ellipsometer bietet, das sogenannte Imaging. Dies ermöglicht einerseites die konkrete Auswahl des Messbereichs auf der Probe. Für eine quantitative Analyse von Prozessen an planaren Oberflächen eignen sich insbesondere ellipsometrische Maps, die entgegen der numerischen Daten der ROIs als 2D-Array aufgenommen werden. Dazu wurde neben einer Optimierung der Sofware (Ep4-Control) und seitens der Kamerasteuerung (Belichtungszeit, Bildrate) besonderer Wert auf die Auswertung der produzierten Bilder beziehunsgweise Maps gelegt (DataStudio). Im Vergleich zur Ep3 besteht bei der Ep4-Steuerung die Möglichkeit, die Belichtungszeit der Kamera zu erhöhen. Somit können auch Maps unter Verwendung des 20xbeziehunsgweise 50x Objektives generiert werden. Denn besonders bei einer hohen lateralen Auflösung reichte zuvor das detektierte Licht nicht aus, um auswertbare Maps zu detektieren. Selbst schwach reflektierende Substrate wie Glas können durch geeignete Einstellungen der Kamera aufgenommen werden.

\subsubsection{Quantitative Analyse}

Anhand von Maps können Proben quantitativ untersucht werden. Dazu werden die jeweiligen Maps in Form von Histogrammen dargestellt. Anhand dessen lassen sich anschließend Daten wie Mittelwert, Halbwertsbreite beziehunsgweise Standradabweichung des jeweiligen Parameters durch Anlegen einer Gaussfunktion bestimmen.

\subsubsection{Micro-contact Printing}

Im Bereich der Oberflächenfunktionalisierung stellen self-assembled monolayer (SAM) eines der wichtigstens Tools dar. Dabei gibt es verschiedene Methoden, um die Oberflächen mit den Molekülen zu beschichten. Neben der spontanen Aggregation der Moleküle aus einer Lösung beziehungsweise aus Dampf an der gewünschten Oberfläche hat sich die „contact printing“-Methode als geeignet erwiesen, um ein Mikropattern zu erzeugen [60]. Mittels abbildender Ellipsometrie wurde der Prozess des 
„micro-contact printing“ von OTS auf $\mathrm{SiO}_{2} / \mathrm{Si}$ (100) untersucht. Die Herstellung der Proben wird im Kapitel 6 beschrieben. Es wurden 100 Delta- und Psi-Maps (10x Objektiv, $\mathrm{AOI}=50^{\circ}, \lambda=500 \mathrm{~nm}$ ) aufgenommen (4 Proben, 25 Positionen pro Probe). Ein exemplarisches Linienprofil sowie das dazugehörige Histogramm einer zugeschnittenen Delta-Map zeigt Abbildung 4.1.
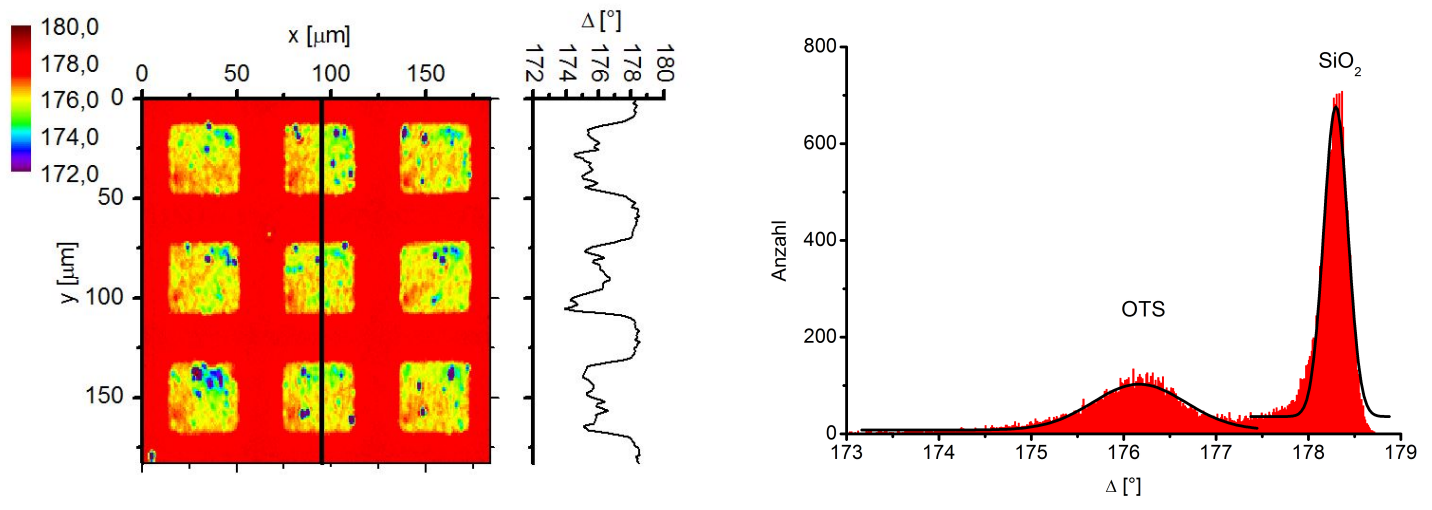

Abbildung 4.1 Delta-map OTS SAM als Linienprofil (links) und das dazugehörige Histogramm (rechts).

Die Darstellung von ellipsometrischen Kontrastbildern und Maps in Form von Linienprofilen beziehungsweise Histogrammen wurde ebenfalls ins DataStudio implementiert. Die Maps wurden durch eine geeigneter Modellierung des Schichtsystems (Ep4-Model) ausgewertet. Dabei wurden die optischen Konstanten des SiliziumSubstrats und $\mathrm{SiO}_{2}$ aus der Datenbank (Kapitel 7) genutzt. Der Brechungsindex des Silans wurde auf einen Wert von 1.45 gesetzt. Da der ellipsometrische Parameter Delta bei diesem Schichtsystem sensitiver im Hinblick auf die Schichtdicke ist, wurden nur die Delta-Maps mittels Interpolation von Delta (20 Intervalle) in Schichtdickenkarten konvertiert. Die daraus berechnete Schichdickenkarte ist in Abbildung 4.2 als 3D-Plot dargestellt. 


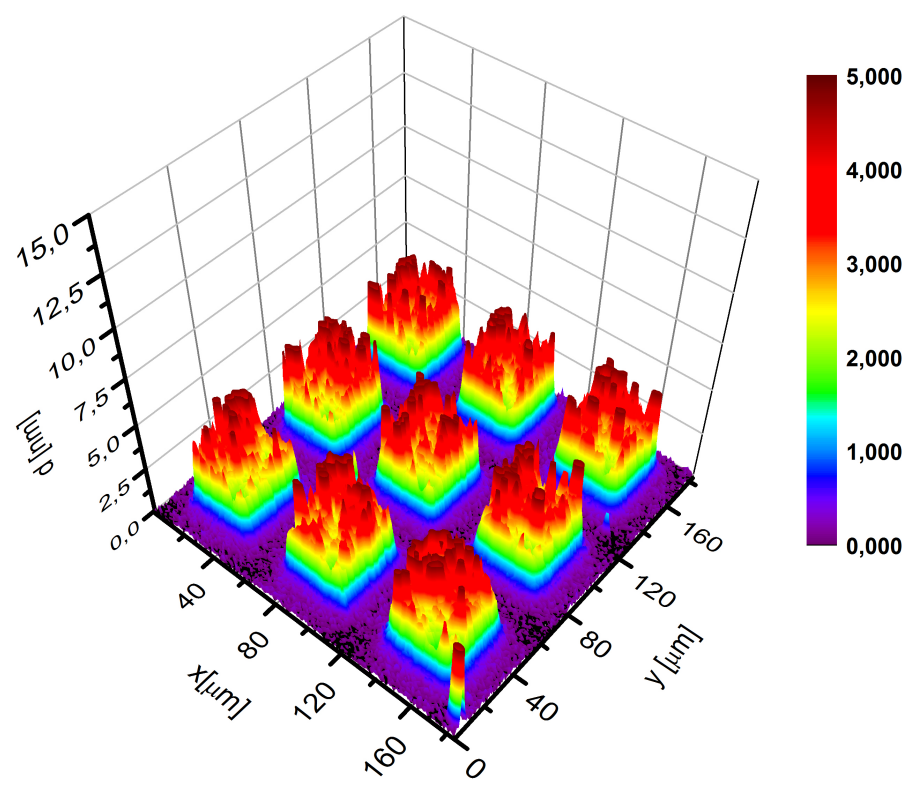

Abbildung 4.2 3D-Plot eines micro-contact printed Pattern von OTS auf $\mathrm{SiO}_{2} / \mathrm{Si}(100)$.

Die Histogramme wurden quantitativ ausgewertet. Der Mittelwert sowie die Standardabweichung sind in Tabelle 4.1 zusammengefasst.

\begin{tabular}{|c|c|c|c|c|}
\hline $\mathrm{c}(\mathrm{OTS})[\mathrm{mM}]$ & $\bar{d}\left(\mathrm{SiO}_{2}\right)[\mathrm{nm}]$ & $\sigma\left(\mathrm{SiO}_{2}\right)[\mathrm{nm}]$ & $\bar{d}(\mathrm{OTS})[\mathrm{nm}]$ & $\sigma(\mathrm{OTS})[\mathrm{nm}]$ \\
\hline \hline 5 & $2.39 \pm 0.25$ & $0.21 \pm 0.08$ & $6.36 \pm 2.15$ & $1.19 \pm 0.80$ \\
\hline 1 & $2.01 \pm 0.24$ & $0.16 \pm 0.04$ & $3.30 \pm 0.88$ & $1.48 \pm 0.80$ \\
\hline
\end{tabular}

Tabelle 4.1 Übersicht Schichtdicke von $\mathrm{SiO}_{2}$ und mikropatterned OTS.

Zusätzlich zur Schichtdicke von OTS konnte stets die Schichtdicke des $\mathrm{SiO}_{2}$ mitbestimmt werden. In der Gesamtheit aller Messungen variiert die Schichtdicke des nativen $\mathrm{SiO}_{2}$ um bis zu $12 \%$. Der Peak der $\mathrm{SiO}_{2}$-Schicht ist jedoch bei jeder Aufnahme relativ schmal im Vergleich zum Peak der OTS-Schicht. Anhand der Daten ist deutlich zu erkennen, dass bei einer Konzentration von $5 \mathrm{mM}$ - im Vergleich zu $1 \mathrm{mM}$ - von OTS in Toluol im Mittel eine fast doppelte Schichtdicke berechnet wird. Hinzu kommt, dass über die gesamten Proben die Schichtdicke bei höherer Konzentration stärker variiert als bei geringerer Konzentration. Allerdings zeigt die Konzentration keinen Einfluss auf die Breite des Peaks für die OTS-Schicht. Die Schichtdicke bei höherer Konzentration entspricht nicht dem gewünschten Wert einer Monolage.

Es ist davon auszugehen, dass bereits hier die Aggregation der OTS-Moleküle beginnt, so dass keine einzelnen Moleküle an der Oberfläche reagieren. Der Wert bei geringer Konzentration ( $1 \mathrm{mM}$ ) entspricht schon eher dem zu erwartenden Wert des langkettigen aliphatischen Silanmoleküls $\left(\mathrm{d}_{\text {Lit. }}=2.6 \pm 0.2 \mathrm{~nm}\right)[58]$. 


\title{
4.1.1.2 Laserablation
}

Auch die nachträgliche Bearbeitung (Mikrostukturierung) von dünnen Filmen an planaren Substraten wurde mittels abbildender Ellipsometrie untersucht. Dieses Unterkapitel befasst sich mit der ellipsometrischen Untersuchungen an dünnen Filmen von Polymethoxymethacrylat (PMMA) auf $\mathrm{SiO}_{2} / \mathrm{Si}$ (100), der mittels Laserablation mikrostrukturiert wurde. Der Herstellungsprozess wieß neben einer Variation der Leistung von Laserpulsen (100 mJ, 150 mJ, $200 \mathrm{~mJ}$ und $500 \mathrm{~mJ}$ ) ebenfalls verschiedene Wiederholungen pro Position (1,3, und 5) auf. Es wurden an den jeweiligen 12 Positionen Delta- und Psi-Maps aufgenommen $\left(\mathrm{AOI}=50^{\circ}\right.$, Lambda $=500 \mathrm{~nm}$, 50x Objektiv). Die optischen Parameter für PMMA wurden aus der bestehenden Datenbank genutzt um die Delta-Maps in Schichtdickenkarten zu konvertieren [34]. Analog zur vorherigen Anwendung wurde auf die Dispersionen für $\mathrm{SiO}_{2}$ und $\mathrm{Si}$ (100) aus der bestehenden Datenbank zurückgegriffen. In Abbildung 4.3 ist die erhaltene Schichtdickenkarte (100 mJ, 5 Pulse) des mikrostrukturierten PMMA-Films in Form eines 3D-Plots und das dazugehörige Histogramm dargestellt.
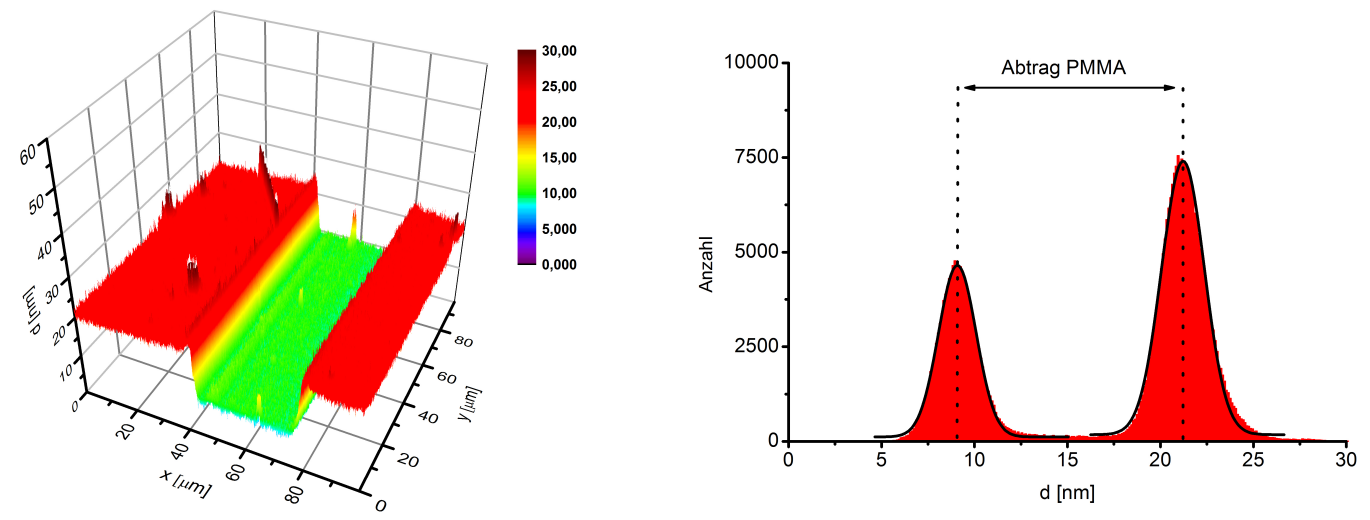

\begin{abstract}
Abbildung 4.3 3D-Plot der erhaltenen Schichtdickenkarte des mikrostruktuierten PMMA-Films (100 mJ, 5 Pulse) auf $\mathrm{SiO}_{2} / \mathrm{Si}$ (100) und Histogramm inklusive des Abtrags an PMMA durch Laserablation.
\end{abstract}

Jede Schichtdickenkarte wurde mittels statistischer Analyse ausgewertet. Die Werte der jeweiligen Peaks (Mittelwert, Standardabweichung) der Histogramme sowie der Abstand zwischen den Peakmaximas, die dem Abtrag an PMMA entsprechen, wird in Tabelle 4.2 gezeigt. Wie zu erwarten, steigt mit zunehmender Anzahl an Laserpulsen der Abtrag an PMMA. Die Ablationstiefe pro Puls nimmt mit steigender Pulsanzahl ab. Die Energie des Laserpulses zeigt im Bezug auf die Ablationstiefe einen exponentiellen abklingenden Effekt. Die Werte bei einer Pulsenergie von $500 \mathrm{~mJ}$ weichen von den Werten ab, die man bei gleichbleibendem Verlauf der Ablationstiefe erwartet. Genauer zeigen die Postionen einen geringen Abtrag an PMMA im Vergleich der Position mit einer Pulsenergie von 200 mJ unabhängig von der Pulsanzahl. 


\begin{tabular}{|c|c|c|c|c|c|c|}
\hline PulsanzahlEnergie & $\begin{array}{c}d(\text { Peak } \\
{[\mathrm{mJ}]}\end{array}$ & $\sigma[\mathrm{nm}]$ & $\begin{array}{c}d \text { (Peak } \\
2)[\mathrm{nm}]\end{array}$ & $\sigma[\mathrm{nm}]$ & $\begin{array}{c}\text { Abtrag } \\
\text { PMMA } \\
{[\mathrm{nm}]}\end{array}$ \\
\hline \hline 1 & 100 & 13.91 & 1.37 & 21.84 & 0.94 & 7.93 \\
\hline 1 & 150 & 14.99 & 1.28 & 23.21 & 0.75 & 8.21 \\
\hline 1 & 200 & 11.68 & 0.90 & 23.34 & 0.83 & 11.66 \\
\hline 1 & 500 & 9.18 & 0.65 & 21.67 & 0.70 & 12.49 \\
\hline 3 & 100 & 11.58 & 1.01 & 22.60 & 0.94 & 11.03 \\
\hline 3 & 150 & 9.62 & 0.74 & 22.92 & 0.72 & 13.30 \\
\hline 3 & 200 & 8.79 & 0.66 & 23.28 & 0.76 & 14.49 \\
\hline 3 & 500 & 9.34 & 0.73 & 21.91 & 0.69 & 12.58 \\
\hline 5 & 100 & 9.08 & 0.85 & 21.21 & 0.98 & 12.14 \\
\hline 5 & 150 & 8.42 & 0.70 & 23.07 & 0.69 & 14.65 \\
\hline 5 & 200 & 8.21 & 0.65 & 23.17 & 0.69 & 14.96 \\
\hline 5 & 500 & 8.39 & 0.74 & 23.39 & 0.80 & 14.00 \\
\hline
\end{tabular}

Tabelle 4.2 Abtrag an PMMA in Abhängigkeit der Anzahl an Pulse und Energie.

Eine graphische Darstellung des Abtrags an PMMA wird in Abbildung 4.4 gezeigt.
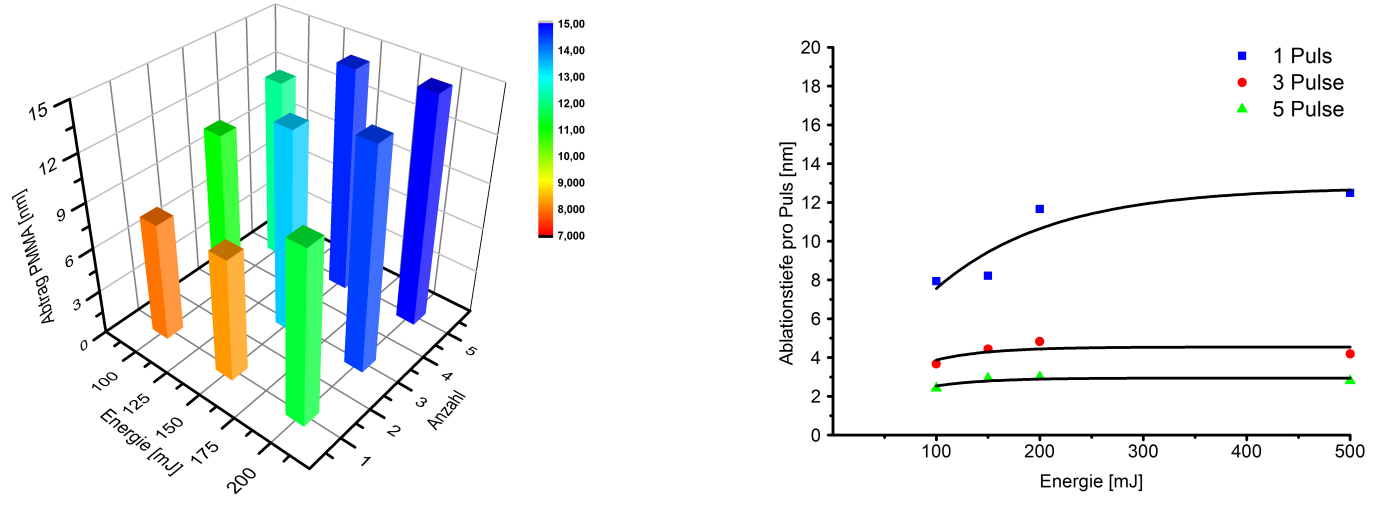

Abbildung 4.4 Darstellung Abtrag von PMMA 


\title{
4.1.1.3 Mikroprägelithografie
}

Neben der Bearbeitung des Schichtmaterials mittels UV-Licht oder Laserablation stellt die Mikroprägelithografie eine einfache und kostengünstige Methode dar, Schichtmaterialien wie Polymere bis hin zu Nanostrukturen zu strukturieren. Anfangs wird das Polymer auf ein Substrat aufgebracht und über die Glasübergangstemperatur erhitzt. Anschließend wird ein mikrostrukturierter Stempel auf das viskose Material gepresst, das aufgrund von Adhäsion in die Ziwschenräume des Stempels gelangt. Der Stempel wird nach dem Erkalten des Materials wieder entfernt und man erhält eine komplementäre Mikrostruktur des Stempels im Schichtmaterial. Aufgrund der Limitierung der lateralen ellipsometrischen Auflösung von $1 \mu \mathrm{m}$ findet die abbildende Ellipsometrie Einsatz im Bereich für Mikrostrukturen.

Als Schichtmaterial wurde in diesem Beispiel amorphes PET verwendet und mittels zwei verschiedener Mikrostrukturen (Linien und hexagonale Strukur) geprägt. Die optischen Parameter des amorphen PETs wurden im Vorfeld durch spektroskopische Untersuchungen an homogenen PET-Schichten auf Siliziumsubstraten bestimmt. Die Dispersion des transparenten Oberflächenmaterials wurde durch eine Cauchy Funktion beschrieben (siehe Kapitel 7).

Von den mikrostrukturierten Proben wurden bei einem Einfallswinkel von $38^{\circ}$ und einer Wellenlänge von 610 nm Delta- und Psi-Maps unter Verwendung des 20x Objektives aufgenommen. Die erhaltenen 2D-Arrays wurden basierend auf dem zuvor angelegten optischen Model - Pixel für Pixel - in Schichtdickenkarten umgerechnet. Ein Auszug der Ergebnisse sind in Abbildung 4.5 gezeigt. Die nahezu maximale laterale ellipsometrische Aufösung des Systems ist darin deutlich zu erkennen [3].
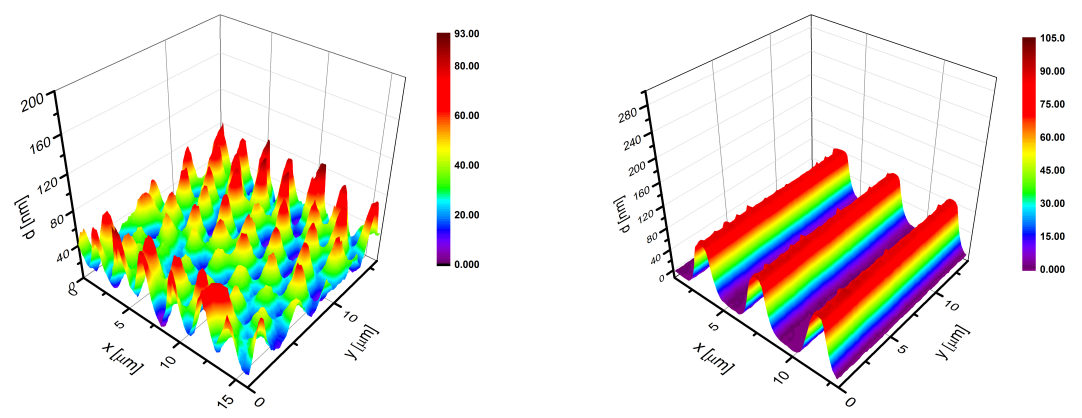

\begin{abstract}
Abbildung 4.5 3D Darstellung der Schichtdickenkarten von mikrostrukturierten PET-Filmen auf Siliziumsubstraten mit hexagonaler (links) und linearer (rechts) Anordnung.
\end{abstract}

Bei der Probe mit der hexagonalen Prägung variiert die Höhe der Mikrostruktur in der Schichtdickenkarte von 45-88 nm. Die Linienprofile hingegen haben keine breite Verteilung in der Höhe mit $90 \mathrm{~nm}$. Zusätzlich wurden AFM-Messungen an beiden Proben durchgeführt (Abbildung 4.6) und werden mit den resultierenden ellipsometrischen Ergebnissen verglichen.

Die Aufnahmen wurden im Bereich von 16x16 um mit einer Datenpunktzahl pro Linie von 526 durchgeführt. Beide Messungen liefern gleiche Ergebnisse bezüglich der 


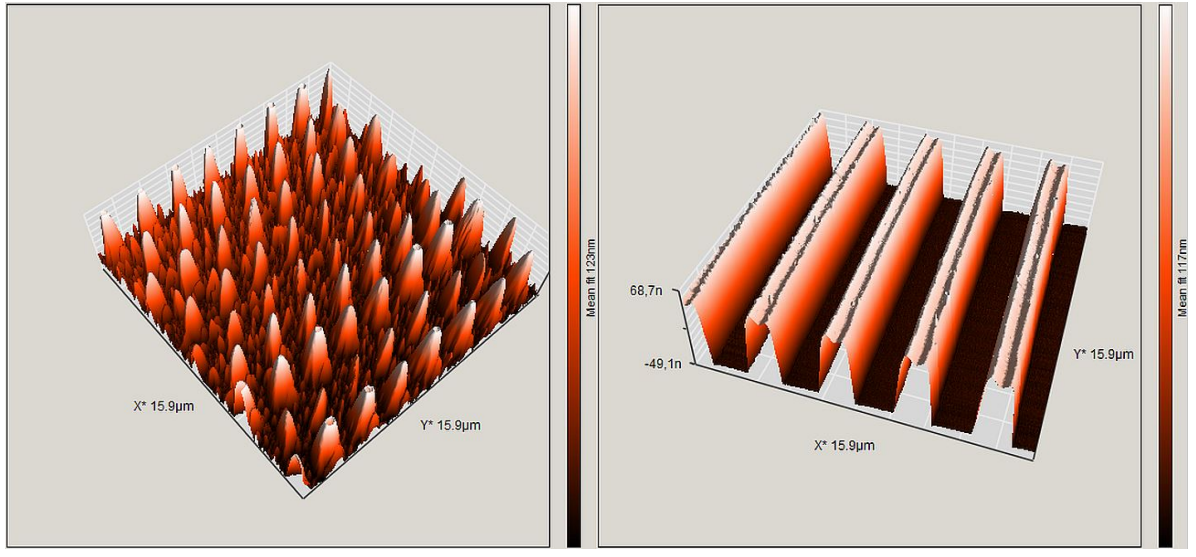

Abbildung 4.6 3D Plot der AFM-Aufnahmen an mikrostruktirierten PET-Film auf Siliziumsubstraten mit hexagonaler und linearer Anordnung.

Höhe der Mikrostrukturen und deren lateraler Dimension. Aufgrund der besseren lateralen Aufösung des AFMs sind zwischen den Erhöhungen zusätzlich Reste des PET's in der hexagonalen Anordnung zu erkennen. Dieser Effekt ist auch bei der abbildenden Ellipsometrie zu erkennen, jedoch wird das Signal aufgrund der Limitierung der lateralen Auflösung gemittelt und man erhält an solchen Stellen keine Schichtdicke von 0. Die Aufnahmen an der linearen Struktur zeigen keinerlei Reste des polymeren Materials zwischen den Erhöhungen, was deckungsgleich mit den Ergebnissen der abbildenden Ellipsometrie ist, da die Schichtdicke zwischen den Linien $0 \mathrm{~nm}$ beträgt. Auf den Linien erkennt man jedoch bei den AFM-Aufnahmen, dass die Seiten etwas höher sind, was auf die Adhäsion zurückzuführen ist. Dieser Effekt kann nicht bei den ellipsometrischen Abbildungen bestätigt werden, was ebenfalls an der lateralen Auflösung liegen kann.

Vergleicht man beide Messtechniken miteinander erkennt man den deutlichen Vorteil der lateralen Auflösung des AFMs. Wobei man nicht außer Acht lassen sollte, dass solche Aufnahmen im Vergleich zur abbildenden Ellipsometrie um ein vielfaches länger dauern und nicht kontaktfrei erstellt werden. 


\subsubsection{4 $\mathrm{As}_{2} \mathrm{~S}_{3}$}

In diesem Anwendungsbeispiel wird ein semi-transparentes Material hinsichtlich seiner optischen Parameter ortsaufgelöst untersucht. Dazu werden auf der Probe drei Bereiche ellipsometrisch betrachtet, bei denen in das Material der Schicht (konstante Schichtdicke) mittels holographischer Interferometrie unterschiedlicher Belichtungszeiten jeweils ein Gitter projiziert wurde. Dies hat jeweils eine lokale Änderung des Brechungsindex' zur Folge.

Zunächst wurde die Schichtdicke des $\mathrm{As}_{2} \mathrm{~S}_{3}$ an jeder der drei Stellen bestimmt. Dazu wurden spektroskopische Messung (10x Objektiv, AOI $=55^{\circ}$, Filterrad, 44 verschiedene Wellenlängen von 360-1000 nm) unter Verwendung des Beamcutters durchgeführt. Die Dispersion des transparenten Substrats wurde durch eine CauchyFunktion beschrieben (Kapitel 7). Das semi-transparente $\mathrm{As}_{2} \mathrm{~S}_{3}$ wurde durch das Tauc-Lorentz Modell (siehe Kapitel 2.6.4.6) parametrisiert. Um eine Variation der Schichtdicke ausschließen zu können, wurden zu den ellipsometrischen Studien additiv AFM Messungen an den selben Stellen durchgeführt.

Bereits bei den ellipsometrischen Kontrastbildern zeigte sich ein großer Unterschied zwischen Aufnahmen mit dem Laser und dem Filterrad. Aufgrund der geringeren Bandbreite des Lasers war das holographische Gitter deutlicher in den ellipsometrischen Kontrastbildern und somit auch in den Maps zu erkennen. Zur Auswertung wurden nur Delta- und Psi-Maps (2x2 Binning, 4 Zonen) unter Verwendung des Lasers aufgezeichnet und ausgewertet (siehe Tabelle 4.3). 
Anhand der spektroskopischen Messungen wurde durch geeignete Modellierung (TaucLorentz, Ep4-Model) für das semi-transparente $\mathrm{As}_{2} \mathrm{~S}_{3}$, neben der Dispersion, auch die Schichtdicke bestimmt. Die jeweilige Schichtdicke wurde - basierend auf den AFM Messungen, dass die Schichtdicke konstant über den betrachteten Bereich ist - als Parameter vorgegeben. Anhand der ellipsometrischen Maps bei $658 \mathrm{~nm}$ wurden anschließend die beiden Parameter $\mathrm{n}$ und $\mathrm{k}$ für jeden Pixel der Map berechnet (point to point). Die resultierenden 3D-Karten sowie die dazugehörigen Histogramme sind in der Tabelle 4.4 abgebildet.

In der Darstellung der Histogramme ist deutlich eine Ausbildung von zwei Peaks zu erkennen, somit ist das holographisch projizierte Gitter im Brechungsindex wiederzufinden [47]. Sowohl die Abstände des Gitters $(20=\mu \mathrm{m})$ als auch der erwartete Einfluss der Belichtung ist deutlich zu erkennen. Der Absorptionskoeffizient zeigt jedoch keine solche Aufsplittung der Werte. Eine Übersicht über die Schichtdicke, die Lage der Peaks, die Aufspaltung des Brechungsindex' und des Absorptionskoeffizienten sind in Tabelle 4.5 gezeigt.

\begin{tabular}{|c|c|c|c|c|c|}
\hline Belichtung & $\mathrm{d}[\mathrm{nm}]$ & $\mathrm{n}_{1}$ & $\mathrm{n}_{2}$ & $\Delta \mathrm{n}$ & $\mathrm{k}$ \\
\hline \hline unter & $966.2 \pm 0.2$ & $2.576 \pm 0.003$ & $2.594 \pm 0.006$ & $0.018 \pm 0.003$ & $0.0056 \pm 0.0017$ \\
\hline ideal & $949.4 \pm 0.1$ & $2.577 \pm 0.0002$ & $2.609 \pm 0.0007$ & $0.032 \pm 0.0005$ & $0.0041 \pm 0.0007$ \\
\hline über & $909.9 \pm 0.1$ & $2.568 \pm 0.003$ & $2.590 \pm 0.003$ & $0.022 \pm 0.000$ & $0.0040 \pm 0.0017$ \\
\hline
\end{tabular}

Tabelle 4.5 Schichtdicke und optische Parameter $(\lambda=658 \mathrm{~nm})$ von $\mathrm{As}_{2} \mathrm{~S}_{3}$ bei verschiedenen Belichtungszeiten.

Zunächst ist ersichtlich, dass die Probe generell makroskopisch gesehen einen Schichtdickengradienten besitzt. Zusätzlich zu den holographischen Projektionen zeigt sich die größte Aufsplittung des Brechungsindex' bei idealer Belichtung des Materials. Jedoch zeigt die Belichtung keinerlei sichtbaren Einfluss auf den Absorptionskoeffizienten. Außerdem beeinflusst die Belichtungszeit lediglich die Lage der Peaks im Histogramm, jedoch nicht merklich die Breite, die sich in der Standardabweichung widerspiegelt. Die ermittelten Werte des Brechungsindex' stimmen mit dem in der Literatur beschriebenen überein [53]. 


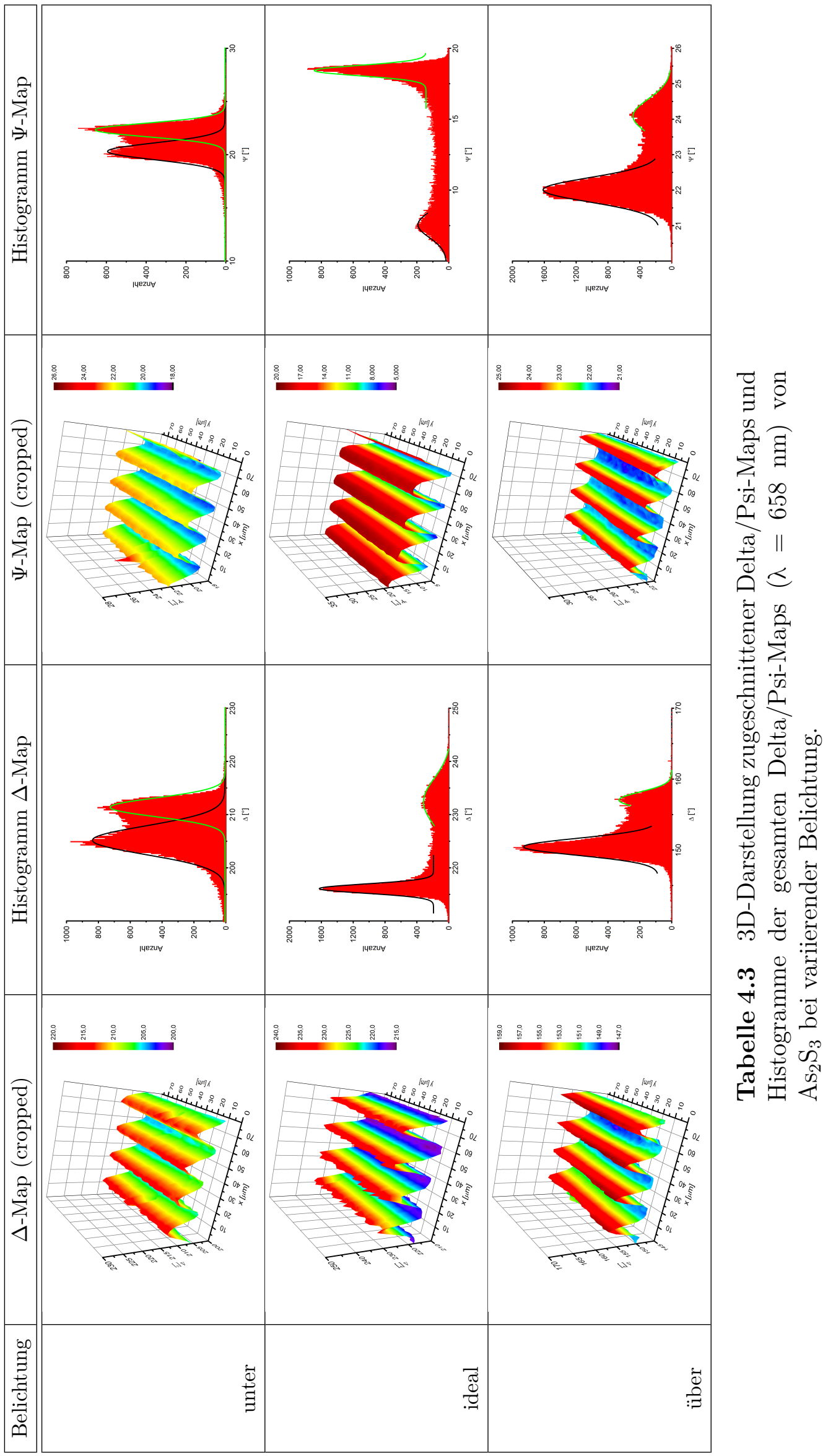




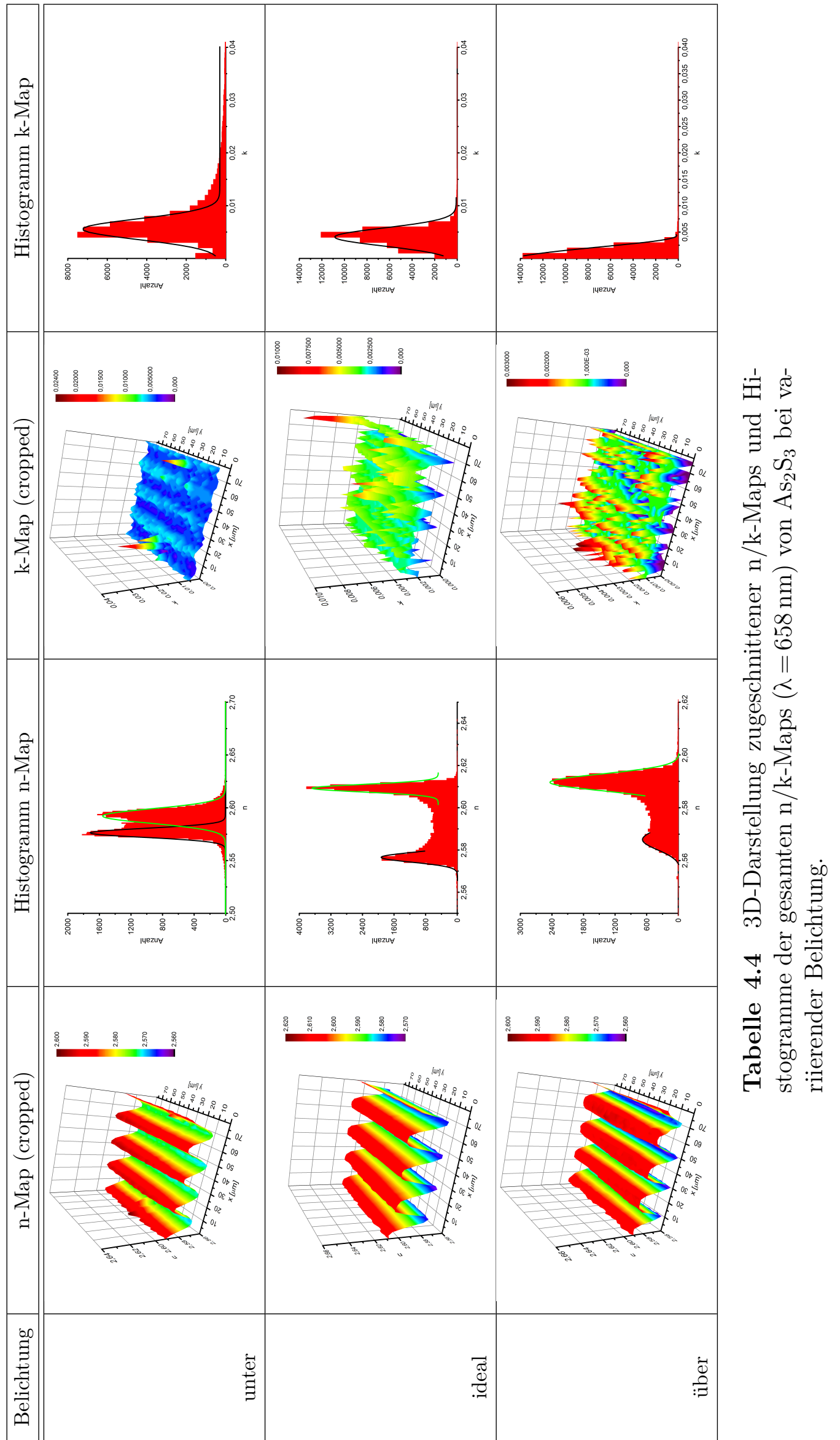




\title{
4.1.1.5 Mikrokristalle
}

Optische Messverfahren wie Lichtmikroskopie und Polarisationsmikroskopie werden zur Visualisierung von mikroskopischen Einkristallen genutzt. Wobei in der Polarisationsmikroskopie zusätzlich die optische Anisotropie der Einkristalle in der Probenebene visualisiert und mittels geeigneter Ausrichtung der Probe die Lage der optischen Achse bestimmt werden kann. Als Erweiterung der Polarisationsmikroskopie können mittels abbildender Ellipsometrie neben der Bestimmung der Schichtdicke, sowohl die Lage der optischen Achse als auch die verschiedenen optischen Parameter innerhalb der Kistalleebene bestimmt werden.

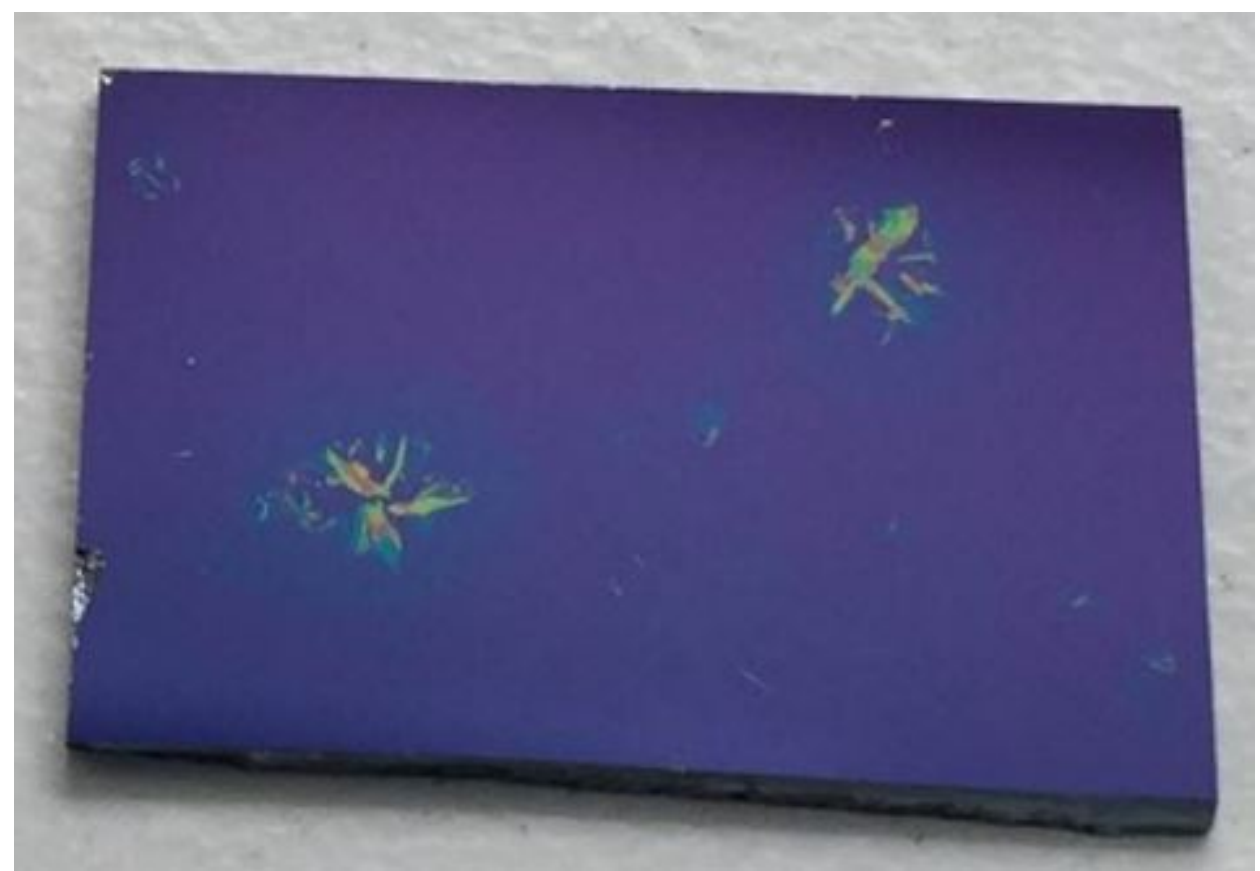

\begin{abstract}
Abbildung 4.7 Fotografie der Einkristalle des ThiophenePhenylen Co-Oligomer auf Siliziumsubstrate mit thermischen $\mathrm{SiO}_{2}$ $(\mathrm{d}=300 \mathrm{~nm})$.
\end{abstract}

Hierzu wurden Mono- und Multilagen von Einkristallen eines organischen Halbleitermaterials mittels abbildender Ellipsometrie spektroskopisch untersucht. Bei dem organischen Halbleiter handelt es sich um ein Thiophen-Phenylen Co-Oligomer (1,4bis(5'-hexyl-[2,2'-bithiophen]-5-yl)benzol) Hex-TTBTT-Hex, welches in Form von Kristallen durch Selbstorganisation unter lösungsmittelhaltiger Atmosphäre auf ein Silizium-Substrat mit einer thermischen $\mathrm{SiO}_{2}$ Schicht $(\mathrm{d}=300 \mathrm{~nm})$ gebracht wurde (Abbildung 4.7).

Zunächst wurde die komplette Probe mittels ellipsometrischen Kontrastbildern bei $\mathrm{AOI}=60^{\circ}$ abgerastert und $\mathrm{zu}$ einer gesamten Karte zusammengesetzt (Abbildung 4.8). Im Vergleich zur Fotografie auf der nur Multilagen zu sehen sind, können ebenfalls dünnere Schichten bis hin zu Monolagen visualisiert werden. Für spektroskopische Untersuchungen wurde anfangs ein mehrschichtiger Bereich ausgewählt 
(gelb), die optischen Parameter mittels geeigneter Modellierung gewonnen und anschließend bei den Messbereichen von Monolagen (blau) angewendet.

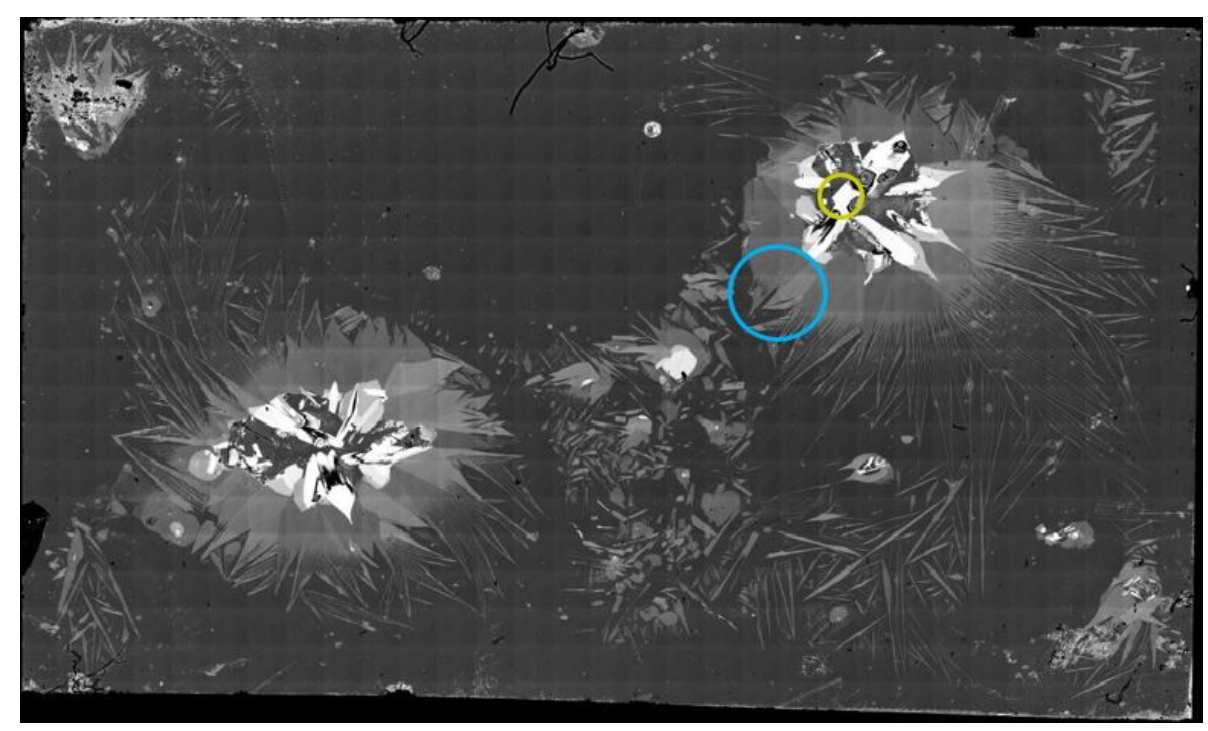

Abbildung 4.8 Ellipsometrische Kontrastkarte der Einkristalle bei $\mathrm{AOI}=60^{\circ}$ und $\lambda=510 \mathrm{~nm}$ inklusive Markierung der Messbereiche der spektroskopischen Untersuchungen an Multilayer (gelb) und Monolayer (blau).

Die Anisotropie des organischen Halbleitermaterials wurde durch die Betrachtung des reflektierten Signals bei p- und s-polarisieren Licht betrachtet. Dazu wurde mit Hilfe eines sogenannten Theta-Stage die Probe um den Messpunkt als Drehpunkt gedreht und das Signal mittels ROI detektiert. Somit wurden bei dem biaxialen anisotropen Systems zwei pseudo-isotrope Punkte gefunden bei denen die Jones-Matrix der Probe einer isotropen Probe entspricht und es zu keiner Kreuzpolarisation zwischen p- und s-Komponente kommt. 

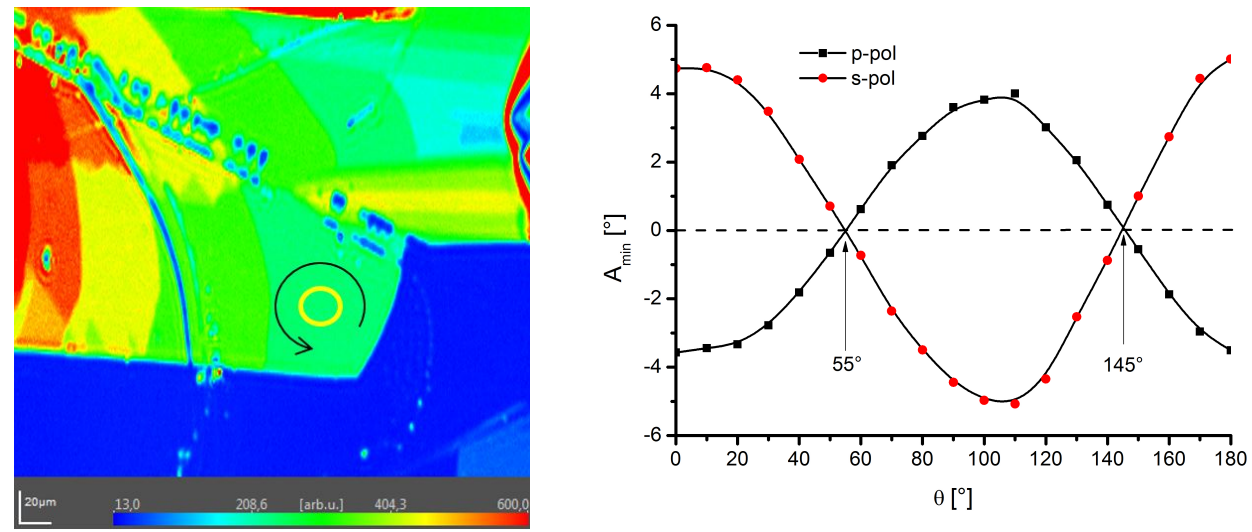

Abbildung 4.9 Links: Ellipsometrische Kontrastkarte des Einkristalls mit Multilagen (AOI $=60^{\circ}, \lambda=600 \mathrm{~nm}$ und $\Theta=55^{\circ}$ ), rechts: graphische Darstellung des detektierten Signals bei p- bzw. s-polarisierten Licht zur Bestimmung des pseudo-isotropen Punktes.

In Abbildung 4.9 ist die Drehung der Probe von 0 bis $180^{\circ}$ mit dem dazugehörigen Minimumsignal des Analysators gezeigt. Als Lichtquelle wurde der Laser $(\lambda=658 \mathrm{~nm})$ verwendet. Zusätzlich sei erwähnt, dass die Minimumposition des Analysators bei spolarisiertem Licht um $90^{\circ}$ versetzt wurde, um die Bestimmung der pseudo-iostropen Punkte zu verdeutlichen. Basierend auf der Kenntnis der pseudo-isotropen Punkte konnten nun die Multilagen des Einkristalls ROI-basiert spektroskopisch charakterisiert werden (Abbildung 4.10). Dabei wurden neben der Variation der Wellenlänge im Bereich von 400-700 nm in $5 \mathrm{~nm}$ Intervallen zusätzlich zwei Einfallswinkel $\left(50^{\circ}\right.$, $60^{\circ}$ ) gewählt.
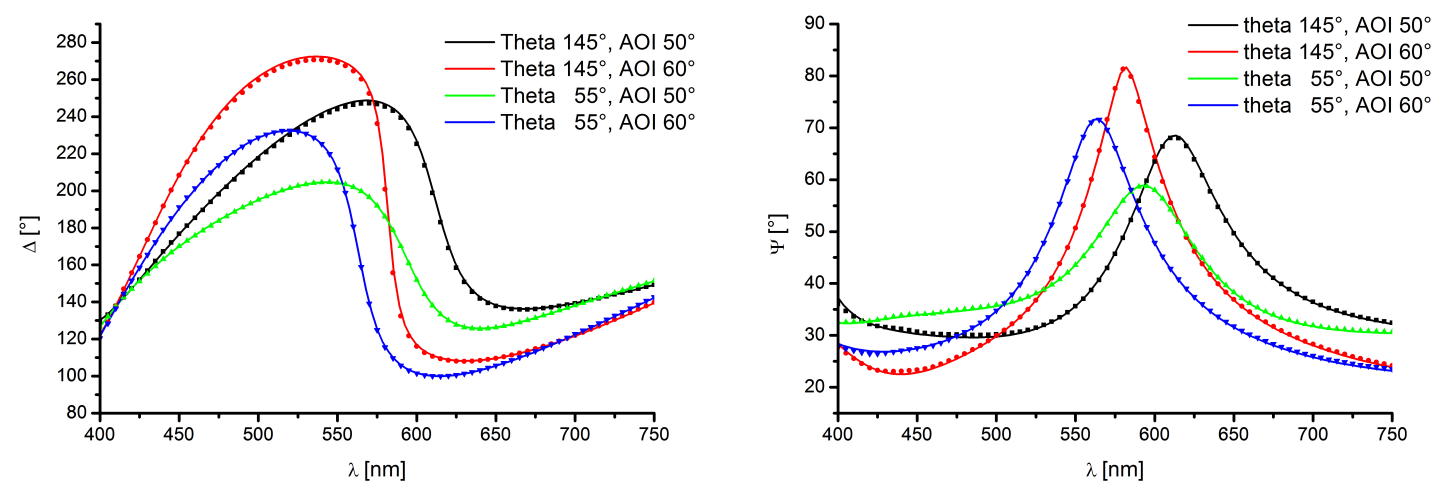

Abbildung 4.10 Graphische Darstellung von Delta/PsiWellenlängenspektren $(\lambda=400-750 \mathrm{~nm}, 5 \mathrm{~nm}$ Intervalle) bei zwei verschieden Einfallswinkeln $\left(\mathrm{AOI}=50^{\circ}, 60^{\circ}\right)$ an den pseudo-isotropen Punkten $\left(\Theta=55^{\circ}, 145^{\circ}\right)$ von Hex-TTBTT-Hex. 
In den Delta/Psi-Spektren ist neben der Aufspaltung aufgrund des Einfallswinkel ein deutlicher Einfluss der Orientierung der Probe zu erkennen, was sich durch die biaxiale Anisotropie des Einkristalls erklären lässt. Bei der Modellierung der optischen Eigenschaften wurde ein Lorentz-Oszillator verwendet. Die sich ergebende Dispersion in den drei Raumrichtungen wird in Abbildung 4.11 gezeigt und woraus für die Multilage eine Schichtdicke $\mathrm{d}=41.2 \pm 0.2 \mathrm{~nm}$ resultiert.

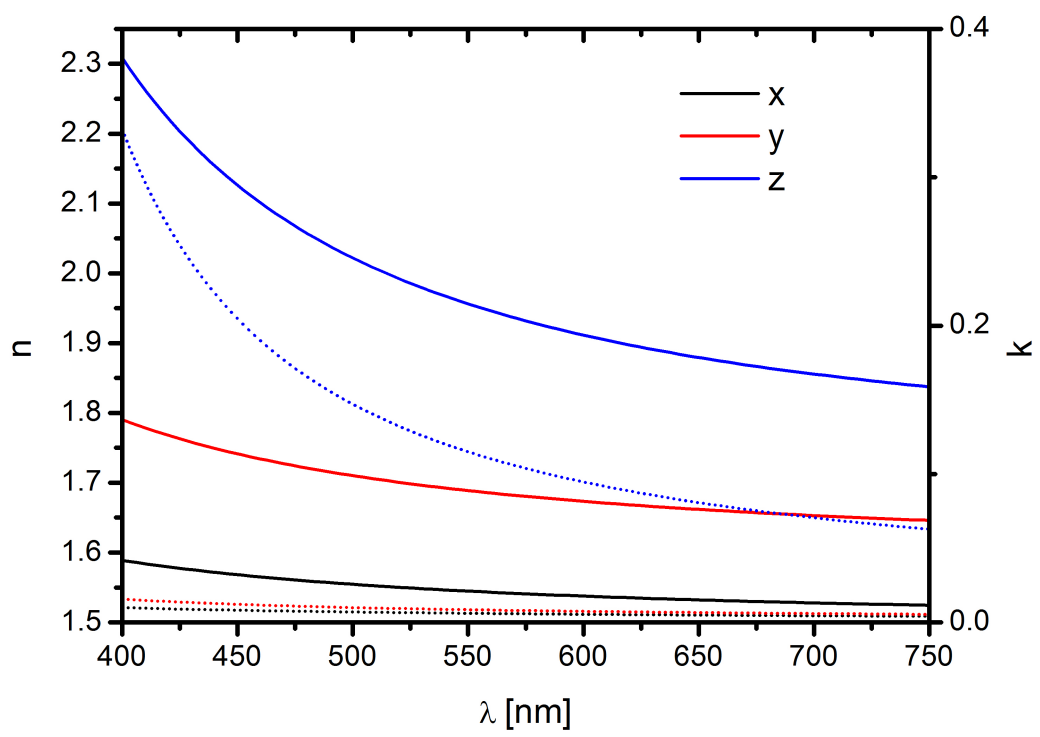

Abbildung 4.11 Dispersion von $\mathrm{n}$ (Linie) und $\mathrm{k}$ (Punkte) des biaxial anisotropen organischen Halbleitermaterials Hex-TTBTT$\operatorname{Hex}(\mathrm{d}=41.2 \pm 0.2 \mathrm{~nm})$.

Nach der Bestimmung der optischen Parameter des biaxialen anisotropen Schichmaterials wurden diese auf die spektroskopischen Maps angewendet und eine Schichtdickenkarte des mehrschichtigen Einkristalls - Pixel für Pixel - berechnet (Abbildung 4.12). Durch eine quantitative Histogrammanalyse der Schichtdickenvariation des Einkristalls ergab sich der Wert für eine molekulare Schicht $\left(\mathrm{d}_{\text {molekulare Schicht }}=3.49 \pm 0.2 \mathrm{~nm}\right)$. 

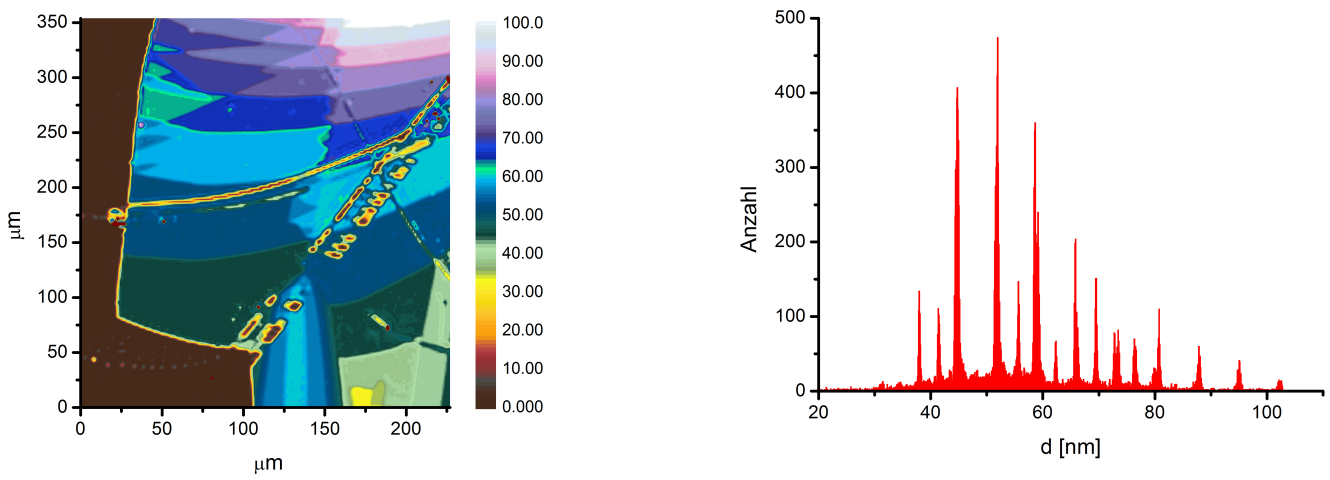

Abbildung 4.12 Schichtdickenkarte des mehrschichtigen Einkristalls $\left(\Theta=145^{\circ}\right)$, sowie die quantiative Analyse zur Bestimmung der molekularen Schicht $\left(\mathrm{d}_{\text {molekulare Schicht }}=3.49 \pm 0.2 \mathrm{~nm}\right)$.

Analog zu den bisherigen Betrachtungen der Multilagen des biaxial anisotropen Material wurden analoge Messungen zur Bestimmung der pseudo-isotropen Punkte an Monolagen (Abbildung 4.13) durchgeführt. Dabei zeigt die grafische Darstellung einen leichten Offset zum Nullpunkt, wobei die Ursache dieses Effekts noch nicht geklärt ist.
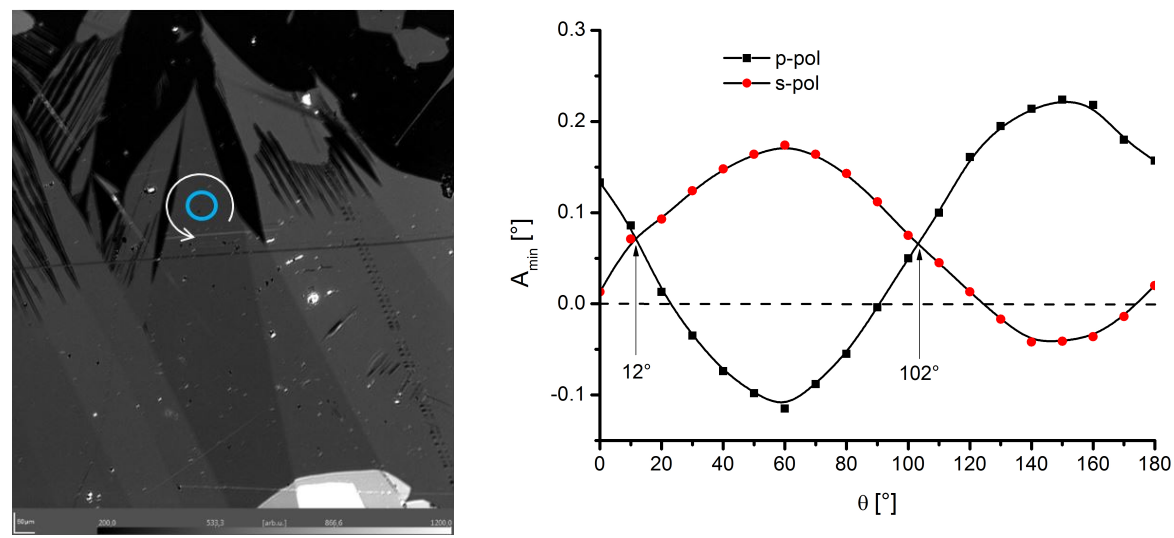

Abbildung 4.13 Links: Ellipsometrische Kontrastkarte des Einkristalls mit Multilagen ( $\mathrm{AOI}=60^{\circ}, \lambda=600 \mathrm{~nm}$ und $\Theta=12^{\circ}$ ), rechts: graphische Darstellung des detektierten Signals bei p- bzw. s-polarisierten Licht zur Bestimmung des pseudo-isotropen Punktes.

Nach der genauen Ausrichtung der Probe wurden Delta maps bei $550 \mathrm{~nm}$ unter einem Einfallswinkel von $50^{\circ}$ aufgenommen und unter Verwendung der zuvor bestimmten optischen Parameter des Materials in eine Schichtdickenkarte konvertiert (Abbildung 4.6). Neben dem Parameter der Schichtdicke wurde ebenfalls der ThetaWinkel als Fitparameter ausgewählt. Da die Schichtdickenkarten als Ergebnis eine homogene Schicht zeigt und keine Variation, wie in den urspünglichen Delta maps, 
handelt es sich vermutlich um einen Versatz von Theta bei den Monolagen, die die unterschiedlichen Delta Werte verursachen. Da aber die Orientierung der Probe ROI basiert durchgeführt wurde, ist auch nur für diesen Bereich die pseudo-isotope Orientierung gewährleistet. Um jedoch die komplette Map in eine Schichtdickenkarte zu überführen, muss dieser mögliche Effekt eines Theta-Offsets der optischen Achsen bei der Modellierung berücksichtigt werden, was durch die Auswahl von Theta als Fitparameter gewährleistet ist. Mittels Histogramm-Analyse wurde anhand der Schichtdickenkarten eine Schichtdicke d Monolage $=3.16 \pm 0.09 \mathrm{~nm}$ generiert. Diese liegt jedoch deutlich unter dem Wert für eine molekulare Schicht bei Multilagen, was eventuell durch eine nicht ideale, komplett gestreckte, Anordnung des Moleküls erklärt werden könnte.

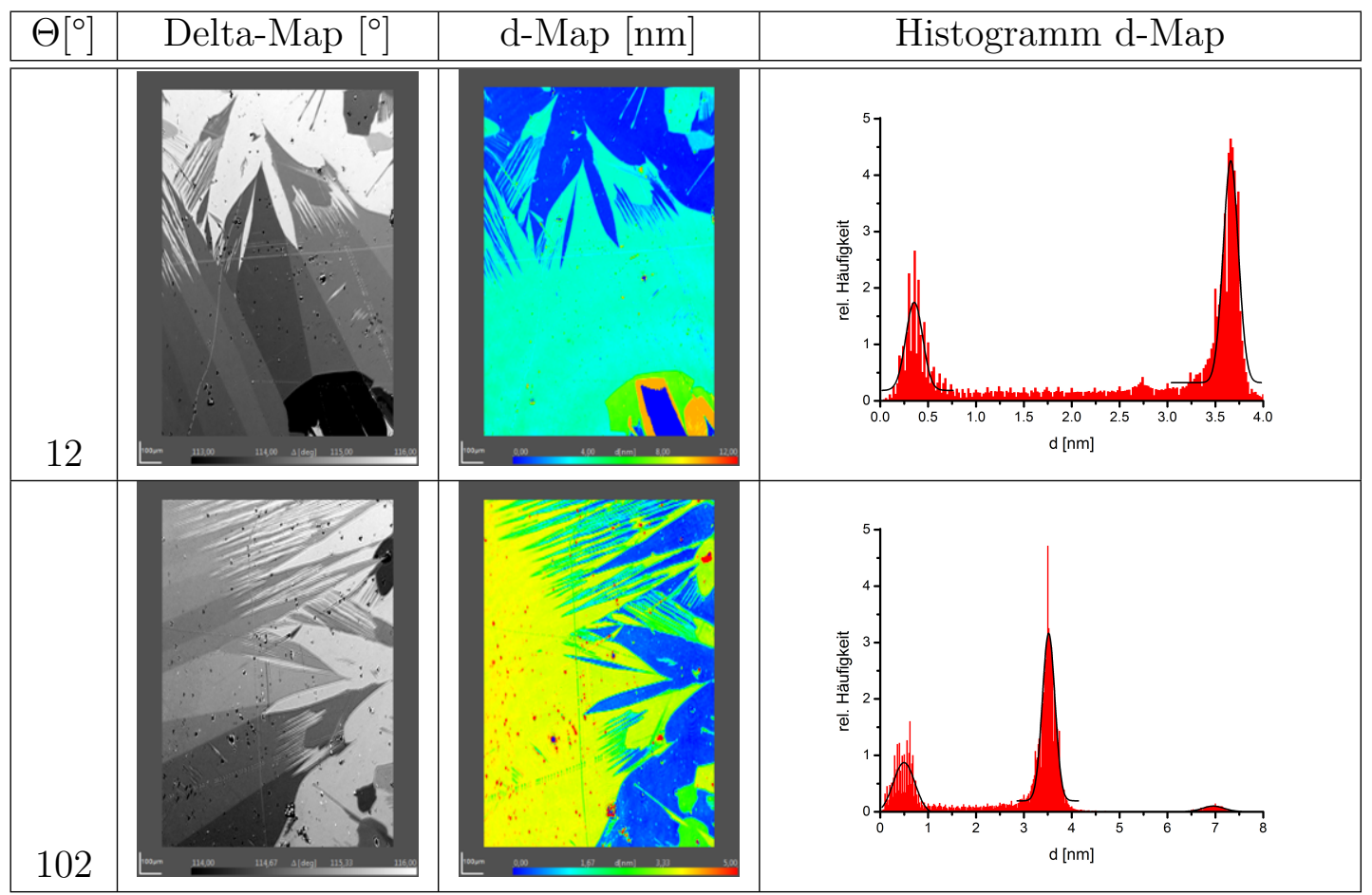

Tabelle 4.6 Delta-Maps (links, AOI $=50^{\circ}, \lambda=550 \mathrm{~nm}$ ) der Monolagen des Einkristalls an den pseudo-istropen Punkten $\left(\Theta=12^{\circ}, 102^{\circ}\right)$ und die resultierenden Schichtdickenkarten (mitte) inklusive Histogramm Darstellung (rechts) zur quantitativen Bestimmung der Schichtdicke der Monolage (d Monolage $=3.16 \pm 0.09 \mathrm{~nm})$.

Um den aus Delta-Maps bei einer Wellenlänge resultierenden Wert für Monolagen zu verfizieren, wurden ebenfalls ROI basierte spektroskopische Messungen $(\lambda=400-$ $750 \mathrm{~nm}, 5 \mathrm{~nm}$ Intervalle) bei zwei Einfallswinkeln $\left(50^{\circ}, 60^{\circ}\right)$ durchgeführt. Die Messergebnisse sowie die simulierten Spketren sind in Abbildung $4.14 \mathrm{zu}$ finden. Dabei ist in den Spektren der Effekt der Anisotroptie des Materials deutlich geringer als bei den Multilagen, aufgrund der kleineren Schichtdicke ( $\left.\mathrm{d}_{\text {Monolage }}=3.09 \pm 0.07 \mathrm{~nm}\right)$. 

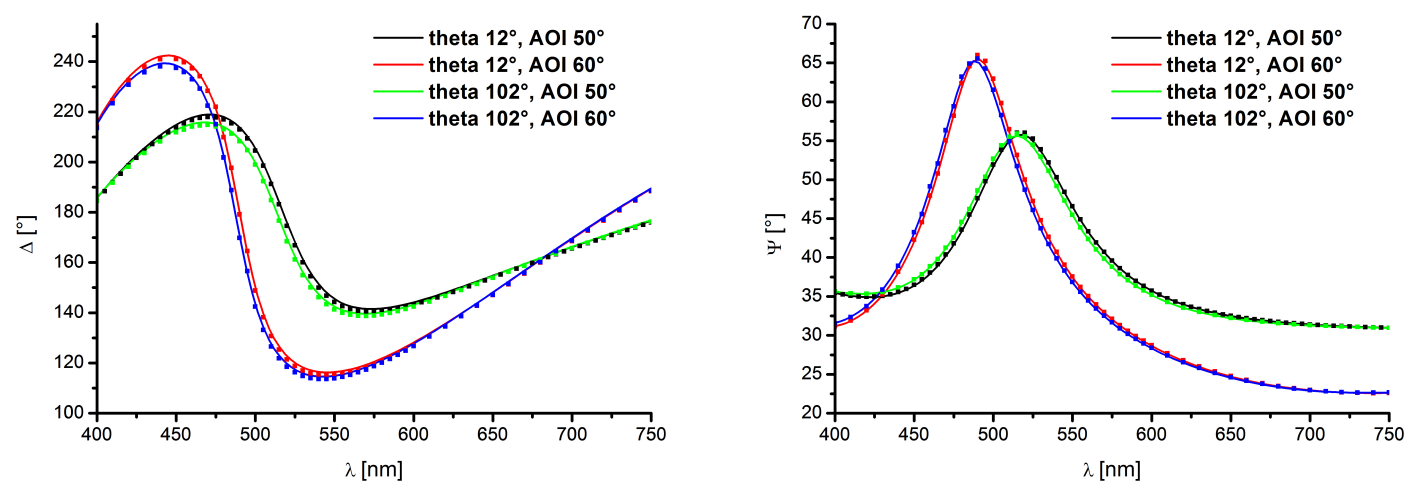

Abbildung 4.14 Graphische Darstellung von Delta/PsiWellenlängenspektren $(\lambda=400-750 \mathrm{~nm}, 5 \mathrm{~nm}$ Intervalle) bei zwei verschieden Einfallswinkeln $\left(\mathrm{AOI}=50^{\circ}, 60^{\circ}\right)$ an den pseudo-isotropen Punkten $\left(\Theta=12^{\circ}, 102^{\circ}\right)$ von Monolagen Hex-TTBTT-Hex $\left(\mathrm{d}_{\text {Monolage }}=3.09 \pm 0.07 \mathrm{~nm}\right)$. 


\subsubsection{Einfluss des Objektives auf die ellipsometrischen Messwerte bei Maps}

In dieser Arbeit wurden je nach Anwendungsfall verschiedene Objektive verwendet. Dabei trat besonders bei Maps bei größeren AOIs in Y-Richtung ein Gradient in den ellipsometrischen Parametern auf. Um den Effekt der Objektive auf den auftretenden Gradienten in Y-Richtung in Abhängigkeit vom Messwinkel zu untersuchen, verglich man Delta- und Psi-Maps bei $500 \mathrm{~nm}$ mit variierendem Einfallswinkel miteinander. Der AOI wurde in $5^{\circ}$ Intervallen von $50-80^{\circ}$ variiert. Bei den Messungen fanden das 2x, 5x und 10x Objektiv Verwendung. Als Schichtsystem dienten zwei Standardproben bestehend aus einer homogenen $\mathrm{SiO}_{2}$-Schicht verschiedener Dicke auf Silizium-Substraten. Mithilfe des Datastudios wurden anhand einer HistogrammAnalyse die Mittelwerte der Maps, sowie die Standardabweichung beim jeweiligen AOI ermittelt. Die sich ergebenden Delta-Psi-Paare für beide Proben sind in Abbildung 4.15 dargestellt und mittels Ep4-Model ergab sich eine Schichtdicke für $\mathrm{SiO}_{2}$ von $30.6 \mathrm{~nm}$ und $116.8 \mathrm{~nm}$.
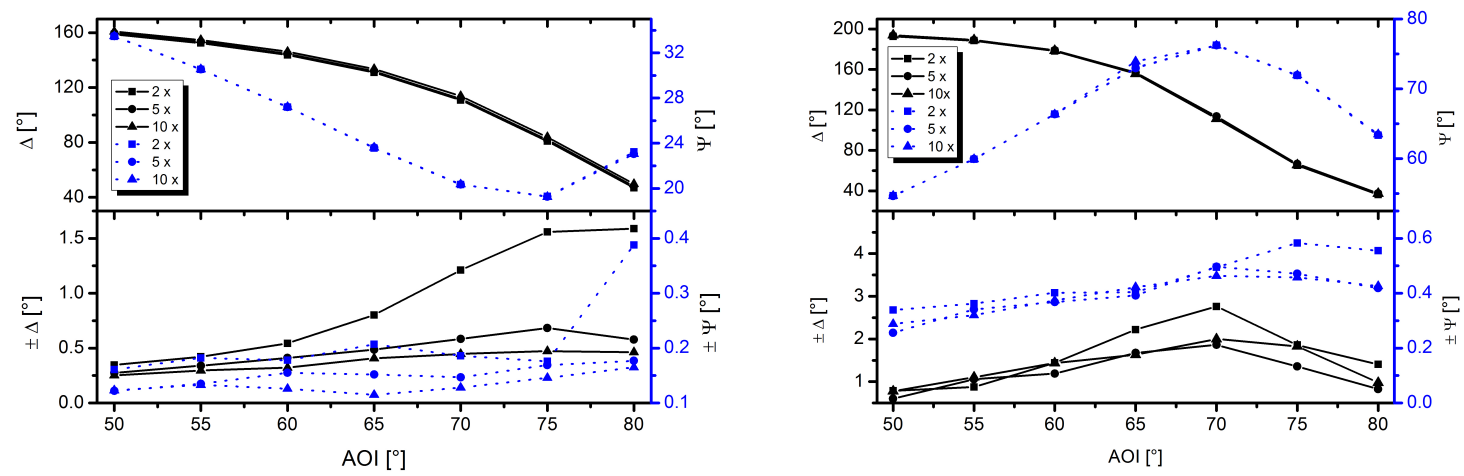

Abbildung 4.15 Delta- und Psi-Mittelwerte und deren Standardabweichung für die Messungen des Standardsysteme vom $\mathrm{SiO}_{2}$ (30.6 nm (links), $116.8 \mathrm{~nm}$ (rechts)) auf Si (100) in Abhängigkeit vom Einfallswinkel.

Die berechneten Mittelwerte der Delta- und Psi-Maps ergeben - wie erwartet - ein klassisches AOI-Spektrum und zeigen keine signifikante Abweichung aufgrund der verschiedenen Objektive. Betrachtet man jedoch die Standardabweichung der DeltaPsi-Werte, die sich aus der Halbwertsbreite der Histogramme generieren lassen, so wird ein deutlicher Zusammenhang zwischen dem verwendeten Objektiv und der Standardabweichung ersichtlich. Dabei zeigt das 2x Objektiv die größte Standardabweichung und nimmt zum 10x Objektiv hin ab. Der Verlauf der Abweichung vom Mittelwerte von Delta bzw. Psi zeigen eine deutliche Abhängigkeit vom generellen Verlauf der Mittelwerte von Delta und Psi. Vergleicht man die Werte der beiden Proben miteinander, erkennt man, dass die Abweichungen bei der Probe mit einer $\mathrm{SiO}_{2}$-Schichtdicke von $116.8 \mathrm{~nm}$ deutlich größer sind als jene der Probe mit $30.6 \mathrm{~nm}$. Daraus lässt sich schlussfolgern, dass es noch einen weiteren Einfluss auf die Abweichung geben muss als das verwendete Objektiv. 
Aufgrund des koliminierten Lichtes auf die Mitte des Lichtspots kommt es zu einer Ausbildung eines Gradienten der ellipsometrischen Werte in Y-Richtung. Um den Effekt des Objektives in Abhängigkeit vom AOI zu betrachten, wurde durch die Mitte der jeweiligen Maps ein Linienprofil in Y-Richtung gelegt und der auftretende Gradient notiert. Die ermittelten Gradienten der Delta- und Psi-Werte sind in der Abbildung 4.16 graphisch dargestellt.
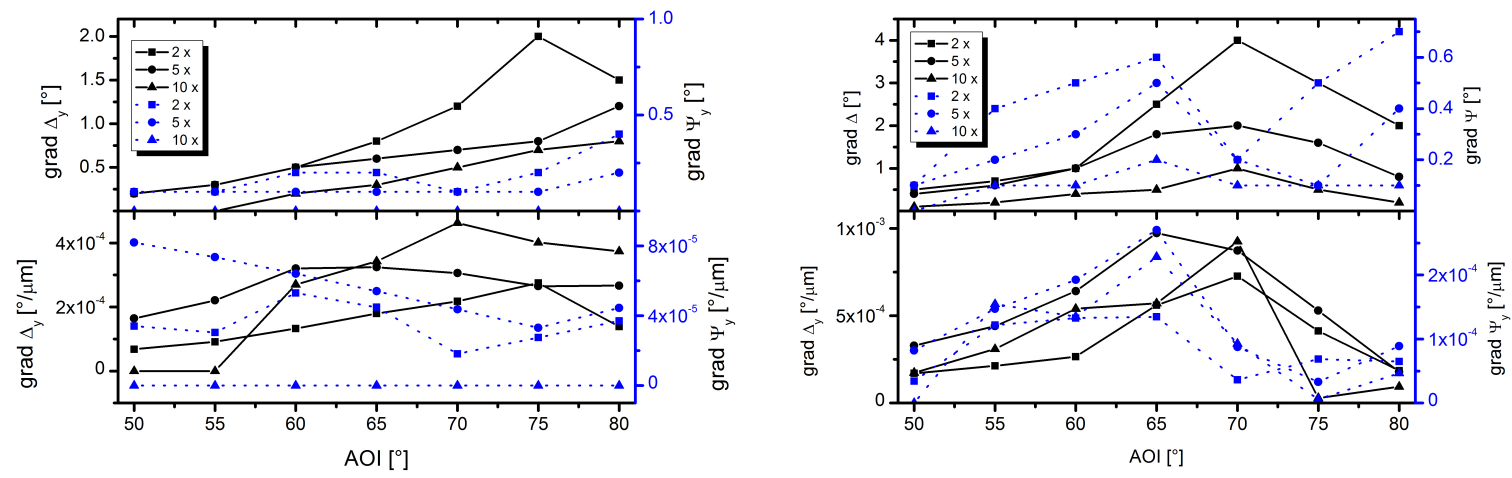

Abbildung 4.16 Gradient in Y-Richtung der ellipsometrischen Maps in Abhängigkeit vom Einfallswinkel für $\mathrm{SiO}_{2}$ (30.6 nm (links), $116.8 \mathrm{~nm}$ (rechts)) auf Si (100).

Bei beiden Schichtdicken für $\mathrm{SiO}_{2}$ konnte beim 2x Objektiv die größten Gradienten in den ellipsometrischen Werten notiert werden. Dieser Effekt lässt sich damit erklären, dass das field of view (FOV) beim 2 x Objektiv am größten ist und bei einer Zunahme von AOI die Streckung des Bildes in Y-Richtung stärker zunimmt (vgl. Gleichung 3.3) im Vergleich zu den beiden anderen Objektiven. Um den Effekt des Sichtfeldes der verschiedenen Objektive zu berücksichtigen, wurde der gemessene Gradient in Y-Richtung durch die Länge der Y-Achse (in $\mu \mathrm{m})$ geteilt. Die relativierten Gradienten sind im unteren Teil der Abbildung gezeigt. Nach der Kompensierung der Streckung des Bildes in Y-Richtung ist kein signifikanter Effekt zwischen den jeweiligen Objektiven zu erkennen. Ein direkten Vergleich zwischen den beiden Proben zeigt jedoch ein leicht unterschiedlichen Verlauf des relativierten Gradienten. In Abbildung 4.17 wird neben der Sensitivität der ellipsometrischen Werte ebenfalls der relativierte, über die Objektive gemittelte Gradient in Y-Richtung für die beiden Proben mit unterschiedlicher $\mathrm{SiO}_{2}$-Schichtdicke gezeigt. 


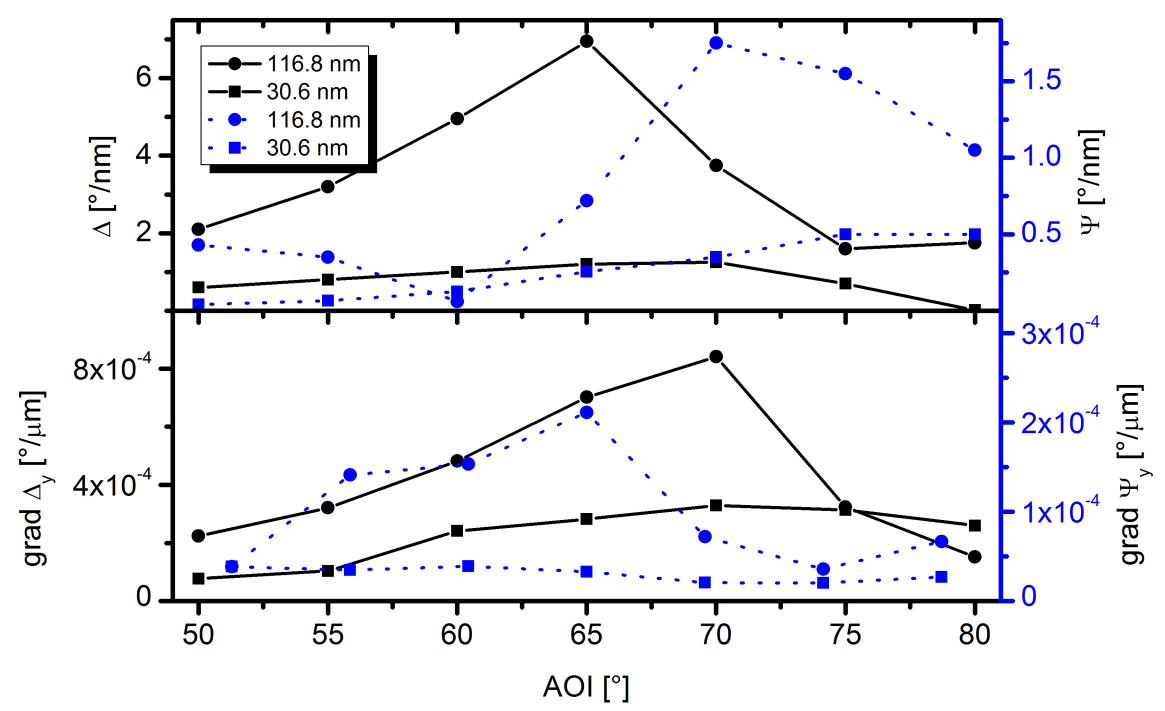

Abbildung 4.17 Sensitivität (oben) und relativierter über die Objektive gemittelter Gradient in Y-Richtung (unten) der beiden Proben bei unterschiedlicher Schichtdicke für $\mathrm{SiO}_{2}$ auf $\mathrm{Si}$ (100) in Abhängigkeit vom Einfallswinkel.

Die Werte für die Sensitivität wurden aus dem Ep4-Model bezogen. Es ist deutlich zu erkennen, dass der Verlauf für die jeweilige $\mathrm{SiO}_{2}$-Schichtdicke dem der Sensitivität in Delta und dem Gradienten in Y-Richtung entsprechen. Auch die Aufsplittung der beiden Probensysteme zueinander ist relativ deutlich zu erkennen. Bezüglich Psi zeigt sich eher ein leichtes inverses Verhalten des Gradienten im Vergleich zur Sensitivität.

Daraus lässt sich schlussfolgern, dass es sich bei der Standardabweichung in Abbildung 4.15 um eine Kombination der beiden Effekte - Streckung in Y-Richtung und Sensitivität - handelt.

Beide Effekte sollten auch bei nicht bildgebenden Ellipsometern auftreten, werden aber aufgrund der fehlenden Bildgebung gemittelt. 


\subsubsection{Ultra-Objektiv}

Eine vielversprechende Entwicklung war die Umsetzung der Scheinflugoptik in die Ep4. Zunächst wurde das Prinzip des Scheinpflugs für die Brewsterwinkelmikroskopie entwickelt. Denn gerade Prozesse im LB-Film-Bereich an der Luft-WasserGrenzfläche - wie Domänenbildung von Lipiden bei der Komprimierung der Barrieren - sind eher schwierig zu verfolgen. Aufgrund der Fluidität der Moleküle an der Wasseroberfläche und deren Bewegung dauert der Scan-Prozess zu lange, als dass verwertbare Abbildungen generiert werden könnten. Das Prinzip der Brewsterwinkelmikroskopie besteht darin, mit p-polarisiertem Licht und einem Einfallswinkel nahe dem Brewsterwinkel des wässrigen Mediums das reflektierte Licht an der Luft-Wasser-Grenzfläche zu detektieren. Bei der reinen Grenzfläche zwischen Luft und Wasser geht der einfallende Lichtstrahl in Totalreflektion über. Befindet sich nun ein Monolage (Lipide [57], Nanopartikel [12], Tenside [35], Flüssigkristalle [8]) ebenfalls im Strahlengang, wird an der dünnen Schicht das Licht reflektiert und von der CCD-Kamera detektiert. Unter Einbeziehung des Brechungsindex' des Materials der dünnen Schicht $\left(\mathrm{n}_{\mathrm{RAFT}}=1.45\right)$ und einer Kalibrierung des Brewsterwinkelmikroskops an der reinen Luft-Wasser-Grenzfläche, kann anhand der Intensität des reflektierten Strahls die Schichtdicke bestimmt werden (Abbildung 4.18).

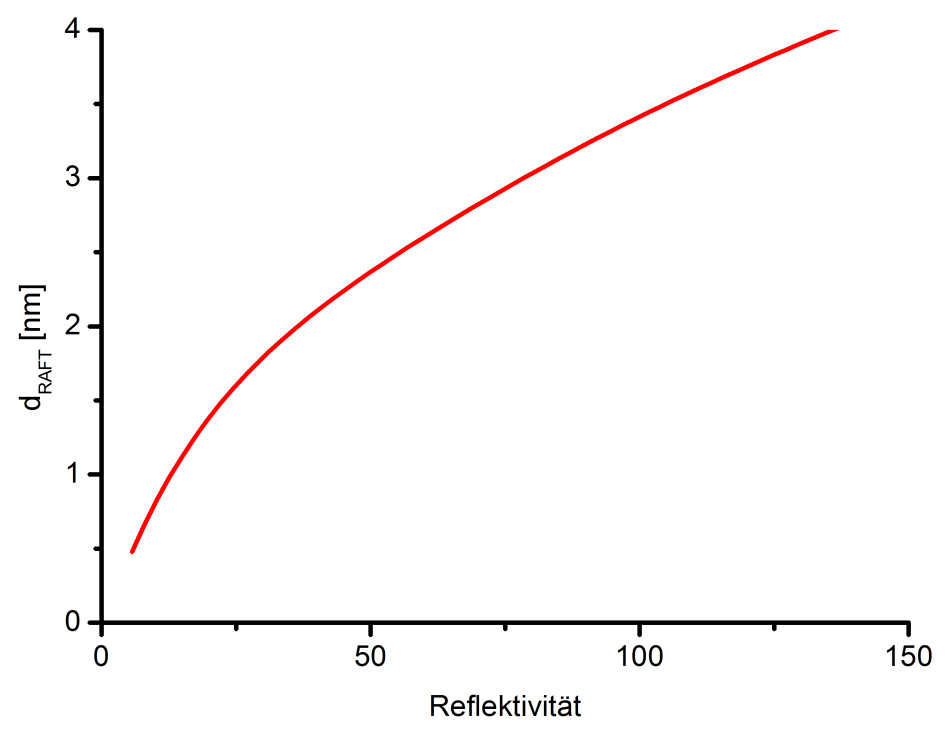

Abbildung 4.18 Kalibrierung der Luft-Wasser-Grenzfläche UltraBAM.

Im Live-Bild erscheinen solche Dünnschichten auf schwarzem Hintergrund (aufgrund der Totalreflexion, siehe Abbildung 4.19) in verschieden Graustufen. Im Anfangsstadium wurden UltraBAM Messungen durchgeführt um die Schichtdicke einer RAFT-Reagenz (4-Cyano-4-[(dodecylsulfanylthiocarbonyl)sulfanyl]pentansäure) zu bestimmen. Dieses RAFT-Agens wurde anschließend dazu verwendet, oberflächengebundene Polymerbürsten zu synthetisieren. Anhand der Messungen sollte eine 
Aussage darüber getroffen werden, ob das RAFT-Agens dazu neigt, Monolagen zu bilden und welche Schichtdicke eine solche Monolage haben würde. Neben den BAMAufnahmen wurde parallel eine Isotherme mittels Wilhelmy-Waage aufgenommen, um zusätzliche Informationen wie die Fläche pro Molekül zu generieren.

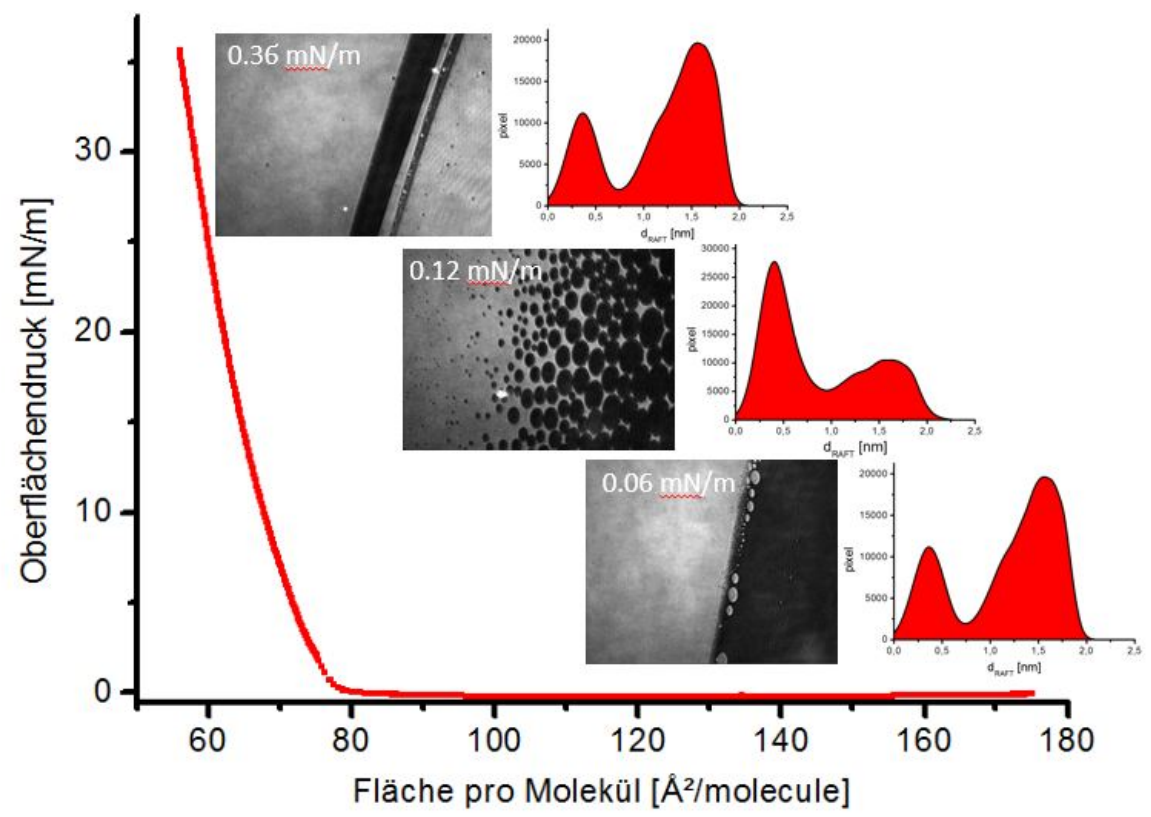

Abbildung 4.19 LB-Isotherme, UltraBAM Bilder sowie Histogramme der berechneten Schichtdicke von 4-Cyano-4[(dodecylsulfanylthiocarbonyl)sulfanyl]pentansäure.

Die UltraBAM-Aufnahmen zeigen schon bei geringen Oberflächendrücken, dass das RAFT-Agens aufgrund der aliphatischen Kette dazu neigt, Monolagen zu bilden. Dabei besitzt eine solche Monolage eine Schichtdicke von $1.24 \pm 0.11 \mathrm{~nm}$. Erst bei höheren Oberflächendrücken über $2 \mathrm{mN} / \mathrm{m}$ ergab sich eine Schichtdicke $\mathrm{d}=3.42 \pm 0.24 \mathrm{~nm}$. Zusätzlich wurde anhand der Isotherme die Fläche pro Molekül (A = $77 \AA$ A/Molekül) für das RAFT-Agenz bestimmt.

Der LB-Film bei geringem Oberflächendruck $(0.5 \mathrm{mN} / \mathrm{m})$ wurde auf ein hydrophilisiertes Silizium-Substrat mit nativer $\mathrm{SiO}_{2}$-Schicht übertragen. Der Übertrag des RAFT-Agens wurde ebenfalls ellipsometrisch untersucht. Dazu wurden bei einem Einfallswinkel von $70^{\circ}$ Delta-Maps mit dem Laser aufgenommen, da insbesondere Delta für dieses Probensystem sensitiv war. In Abbildung 4.20 ist der 3D-Plot einer Delta-Map gezeigt, auf der zudem das Substrat zu sehen ist. 

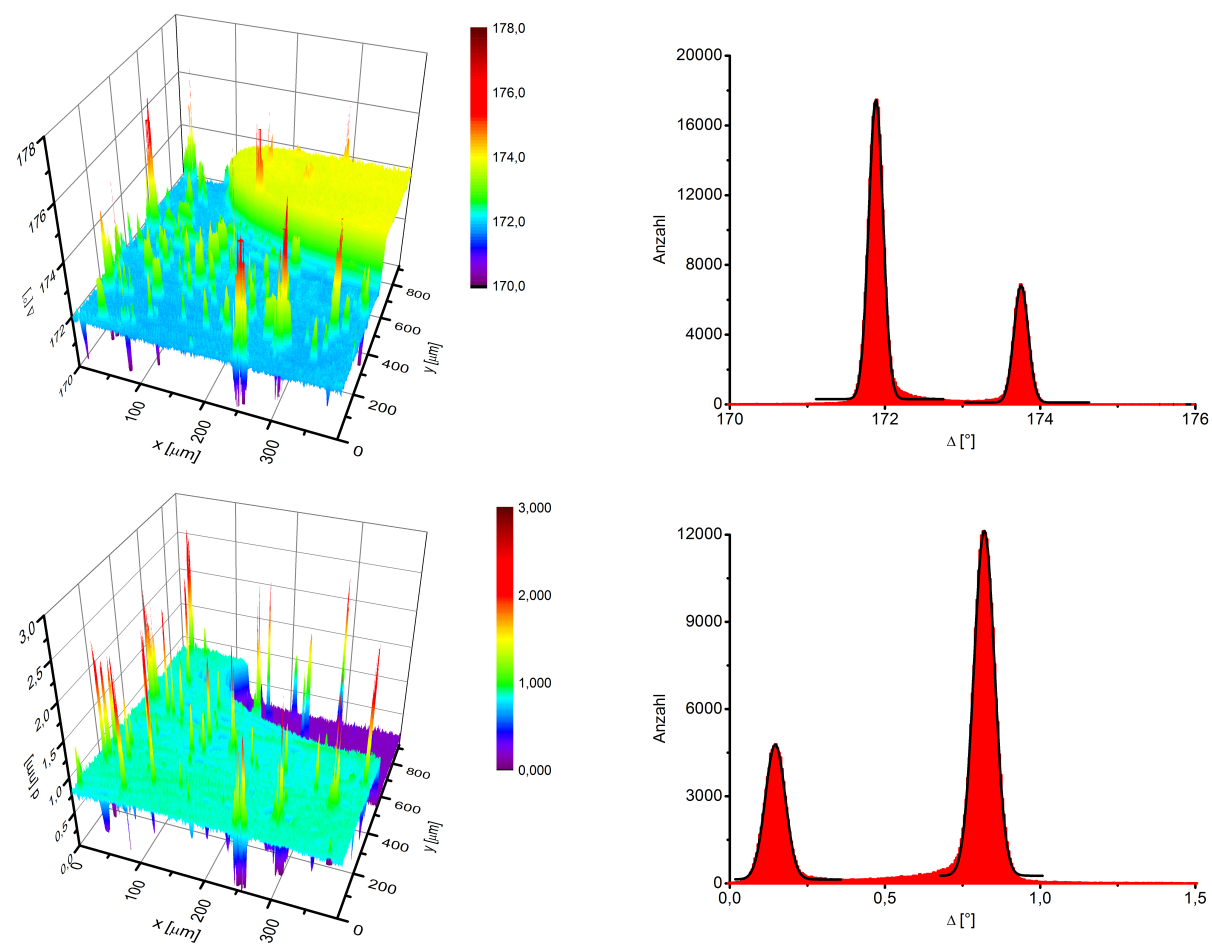

\begin{abstract}
Abbildung 4.20 Delta-/Schichtdicken-Map $\left(\mathrm{AOI}=70^{\circ}\right.$, $\lambda=658 \mathrm{~nm}$ ) und Histogramme des LB-Film Übertrags vom RAFT-Agenz auf $\mathrm{SiO}_{2} / \mathrm{Si}(100)$.
\end{abstract}

Die Delta-Map wurde mittels Interpolation und unter Einbezug eines Brechungsindex' $\mathrm{n}=1.45$ für das RAFT-Agens in eine Schichtdickenkarte umgerechnet. Die optischen Parameter für das Substrat stammen aus der bestehenden Datenbank des Ep4-Models (siehe Kapitel 7). Die statistische Auswertung der Schichtdickenkarte ergab eine Schichtdicke von $\mathrm{d}=0.674 \pm 0.001 \mathrm{~nm}$. Im direkten Vergleich der ermittelten Werte für die Monolage des RAFT-Agens ist die bestimmte Schichtdicke an der Luft-Wasser-Grenzfläche größer und entspricht dem Wert, der in etwa der Länge der aliphatischen Kette entspricht. Analog zu Dodekanthiol befindet sich die aliphatische Seitenkette nicht senkrecht zur Oberfläche. Erst höhere Oberflächendrücke führen zu dickeren Schichten. Wird nun jedoch die Monolage des RAFT-Agens auf ein festes hydrophobes Substrat übertragen, ordnen sich die RAFT-Moleküle eher liegend an.

Da bei der Verwendung der Scheinflugoptik, der Scanning Prozess entfällt, würde die Messzeit beim spektroskopischen Mapping verkürzt werden. Als begrenzender Faktor sei jedoch erwähnt, dass das Ultra-Objektiv nur in einem bestimmten AOI-Bereich genutzt werden kann. Bei Messungen bekannter strukturierter Proben fiel auf, dass das Ultra-Objektiv verglichen mit eine Scannereinheit gleicher Vergrößerung eine geringere Aufösung besitzt. Beim Mapping zeigten Psi-Maps keine scharfen Randbereiche. Für diesen Effekt ist der Analysator verantwortlich, bei dessen Rotation sich ebenfalls das auf die CCD-Kamera projizierte Bild leicht bewegt. Zwar wird dieser Effekt ebenso bei Geräten mit einem Focus-Scanner beobachtet, das Ausmaß ist jedoch deutlich geringer als mit dem Ultra-Objektiv. Um diesen 
gerätespezifischen Effekt zu kompensieren, wurde seitens der Software ein Tool entwickelt, das die Bilder übereinander legt und die vermeintliche Bewegung der Probe im Bild korrigiert.

\subsubsection{Beamcutter}

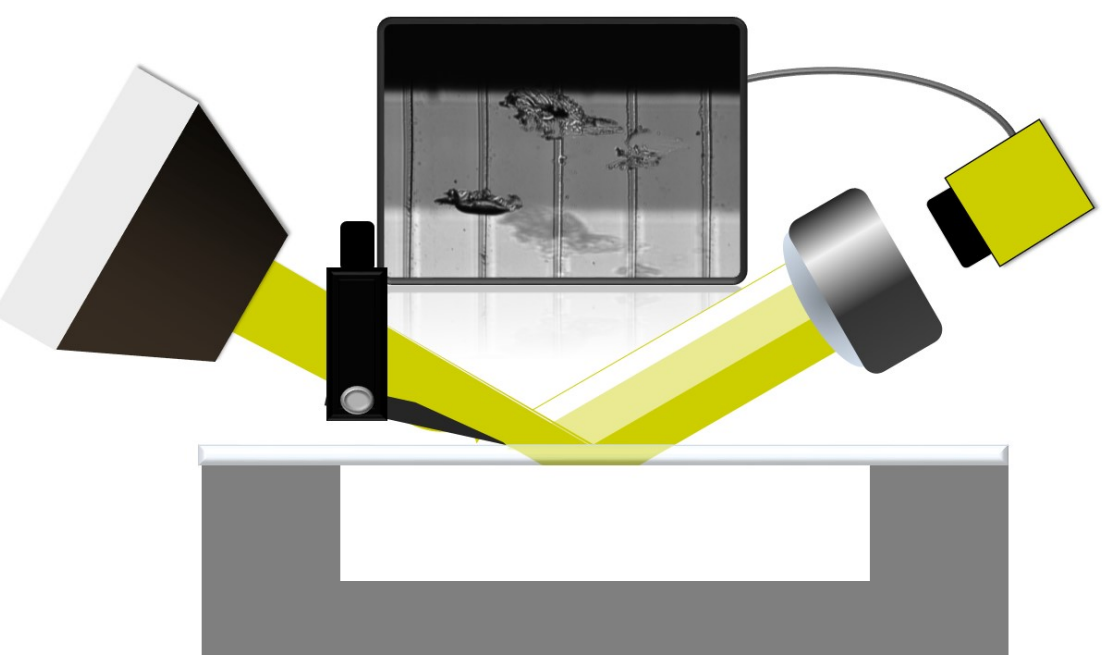

Abbildung 4.21 Prinzip des Beamcutters bei der abbildenden Ellipsometrie inklusive Strahlengang .

Neben intransparenten Substraten wie beispielsweise Silizium oder Gold werden immer häufiger transparente Substrate wie Glas oder Plastikfolien in Beschichtungsvorgängen genutzt. Dabei treffen besonders transparente Folien als flexibles Substrat auf ein steigendes Interesse bei der Industrie. Im Gegensatz zu anderen Mikroskopietechniken $^{29}$ ist die abbildende Ellipsometrie nicht auf bestimmte Substratmaterialien limitiert. Für ellipsometrische Messungen müssen solche Substrate besonders gehandhabt werden. Denn im Gegensatz zu den Standardsubstraten Silizium und Gold wird das einfallende Licht nicht nur an den oberen Grenzflächen reflektiert, sondern ebenfalls an der Substratrückseite. Wird der Rückseitenreflex nicht vermieden oder unterdrückt, kommt es zu einer Überlagerung mit dem generellen Interferenzmuster der Probe. Es gibt mehrere Möglichkeiten, diesen Effekt zu unterdrücken. Bei hinreichend dicken, transparenten Substraten $(>2 \mathrm{~mm})$ ist die Reflektion an der Substratroberfläche deutlich separiert zum Rückseitenreflex. Bei dünneren transparenten Substraten kann man einerseits durch Anrauen der Rückseite, BluTak oder Immersionsöl, den Rückseitenreflex unterbinden [52, 27]. Dabei müssen jedoch die Eigenschaften des Substrats beachtet werden. Bei der Verwendung des Immersionsöls müssen die optischen Parameter dem des Substrats entsprechen, was sich besonders über den gesamten Wellenlängenbereich als schwierig gestaltet. Die beiden anderen Varianten Anrauen der Rückseite oder mit BluTak bekleben sorgen dafür, dass

${ }^{29}$ optische Mikroskopie, Fluoreszenz 
der Rückseitenreflex diffus gestreut wird. Bestenfalls soll die Probe weder behandelt noch mit Substanzen kontaminiert werden.

Bei der Verwendung der abbildenden Ellipsometrie gibt es noch eine weitere Möglichkeit den Rückseitenreflex zu separieren. Mit Hilfe eines Beamcutters (Abbildung 4.21), der sich nahe an der Oberfläche der Probe befindet, wird ein Schatten auf der Probe erzeugt. Dieser Schatten befindet sich im Live-Bild der Kamera und mittels ROIs wird nur das an der Oberfläche reflektierte Licht detektiert [23].

Die Funktion des Beamcutters wurde ebenfalls verbessert, da besonders eine scharfe Abbildung des Schatten nötig ist, um dünne, transparente Substrate $(\mathrm{d}=100 \mu \mathrm{m})$ zu vermessen. Bei der Ep3 war der Beamcutter noch am Laserarm befestigt (Abbildung 4.22 links).

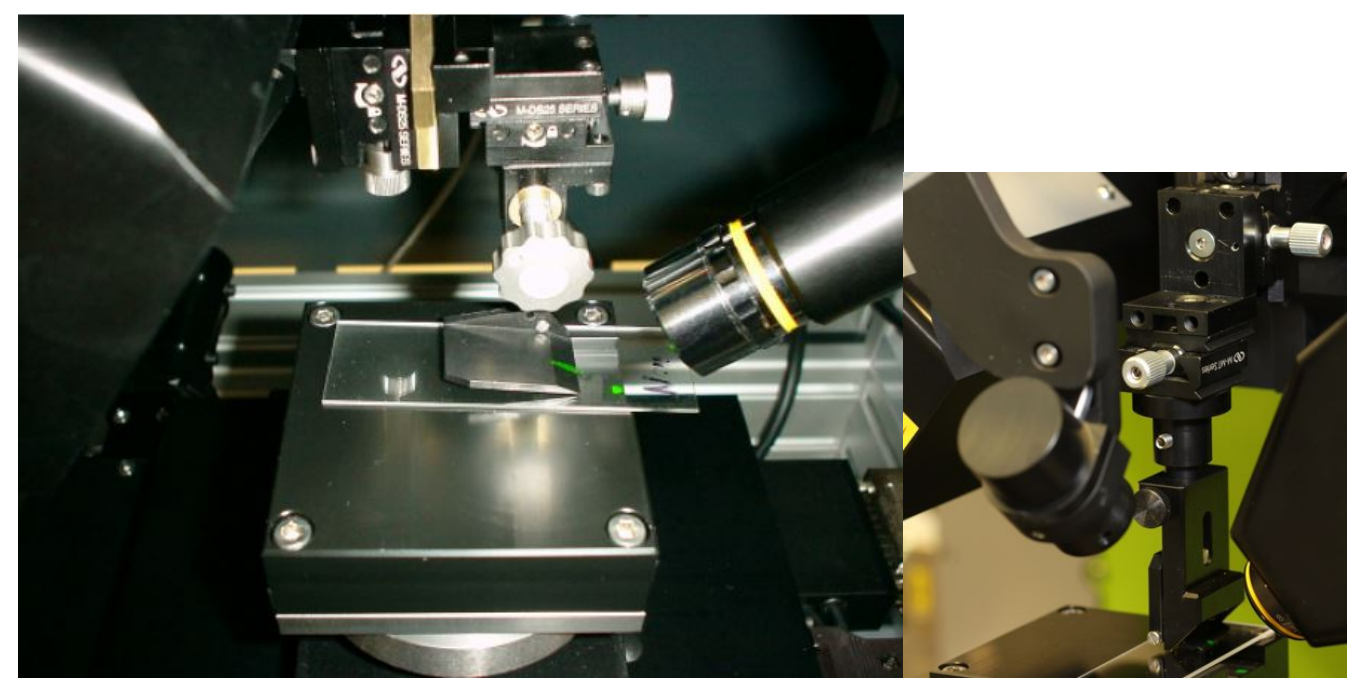

Abbildung 4.22 Beamcutter der Ep3 Generation (links) mit Befestigung am Laserarm und rechts am z-Lift senkrecht zur Probenebene (Ep4) [1].

Durch die Entwicklung eines neuen Alignmentsensors ist die z-Achse über der Probe frei, um mittels z-Lift einen Beamcutter mikrometergenau über der Probe zu positionieren (Abbildung 4.22 rechts). Auch Winkelspektren sind deutlich einfacher aufzunehmen als bei der vorherigen Version des Beamcutters. Denn dort bewegte sich bei Variation des AOI ebenfalls der Beamcutter. Die Anwendung des Beamcutters, auch knife edge illumination genannt, wird am Beispiel von PET-Folie gezeigt.

\subsubsection{PET}

Im Zeitalter der Resourcenknappheit (wie Silizium) und des foranschreitenden Umweltbewusstseins der Weltbevölkerung hat es durch die Entwicklung organischer Solarzellen (plastic solar cell) einen enormen Einschnitt in der Energieindustrie gegeben. Bei den organischen Solarzellen, auch zweite Generation von Photovoltaik genannt, handelt es sich um leitfähige Polymere, die als dünne Schichten auf das 
Trägermaterial aufgebracht werden. Neben geringen Produktionskosten und Materialkosten, im Vergleich zu den herkömmlichen siliziumbasierenden Photovoltaik Zellen, ist besonders die Flexibilität und die damit einhergehende gute Handhabung ein deutlicher Vorteil der organischen Solarzellen. Als Trägermaterial werden immer häufiger transparente Kunstofffolien eingesetzt, die mittels Roll-to-Roll oder Inkjet-Verfahren mit dem leitfähigen Polymergemische beschichtet werden. Dabei steht besonders die spektroskopische Betrachtung der leitfähigen Polymere im Vordergrund, sei es die prozentuale Zusammensetzung der bulk-heterojunctions oder die molekulare Modifizierung des Materials. Neben anderen spektroskopischen Verfahren wird häufig auch die spektroskopische Ellipsometrie zur Charakterisierung leitfähiger Polymere eingesetzt.

Dabei stellt besonders die Charakterisierung von dünnen Schichten auf Folien (transparente Substrate), die eine Dicke von unter $100 \mu \mathrm{m}$ aufweisen, den Anwender klassischer Ellipsometrie vor die Aufgabe, den Effekt der Rückseitenreflexion zu unterdrücken (Kapitel 4.1.3). Alle Proben in diesem Unterkapitel besitzen PET-Folie (HOSTAPHAN, d = $100 \mu \mathrm{m}$ ) als Substrat.

\section{Substrat}

Zunächst wird die Folie bezüglich optischer Anisotropie untersucht. Dazu wird das blanke Substrat auf einem Theta-Stage leicht fixiert. Bei einem AOI $=50^{\circ}$ wurden unter Verwendung des Lasers als Lichtquelle $(\lambda=658 \mathrm{~nm})$ die optischen Komponenten so eingestellt, dass mit p- bzw. s-polarisierten Licht auf die Folie eingestrahlt wurde. Dazu bleibt der Kompensator bei beiden Polarisationszuständen auf $0^{\circ}$. Der Polarisator hingegen wurde für p-Polarisation auf $0^{\circ}$ bzw. für s-Polarisation auf $90^{\circ}$ gesetzt. Nun wird das Intensitätsminimum durch Drehung des Analysators bestimmt. Dieser Vorgang wird für verschiedene Theta-Winkel (Rotation der Probe um die z-Achse) wiederholt. Bei einer ideal isotropen Probe liegt das Minimum des Analysators bei p-Polarisation unabhängig vom Theta-Winkel bei $90^{\circ}$, da es bei gekreuzter optischer Stellung der Komponenten zur Auslöschung des Signals kommt. Analog dazu befindet sich das Intensitätsminimum des Analysators bei sPolarisation des einfallenden Strahls bei $0^{\circ}$.

Der Theta-Scan eines amorphen PET-Film $(\mathrm{d}=50 \mathrm{~nm})$ auf $\mathrm{SiO}_{2} / \mathrm{Si}(100)$ wird in Abbildung 4.23 in Form von Punkten gezeigt. Die Intensitätsminima des Analysators zeigen eine sehr geringe Abweichung vom erwarteten Idealwert. In der Abbildung wird ebenfalls der Theta-Scan der PET-Folie als viereckige Datenpunkte, die mittels gerader Linie verbunden sind, gezeigt. Es ist ganz deutlich eine gewisse Symmetrie des Theta-Scans zu erkennen. Zudem zeigt die Abbildung eine enorme Abweichung des Theta-Scan zwischen amorphen und kristallinen Material. Zusätzlich scheint der Theta-Scan bei der p-Polarisation eine Art von Offset im Gegensatz zur s-Polarisation aufzuweisen. Es wurden ebenfalls Messungen an PolycarbonatFolien durchgeführt, die ein isotropes optisches Verhalten beim Theta-Scan zeigen. 


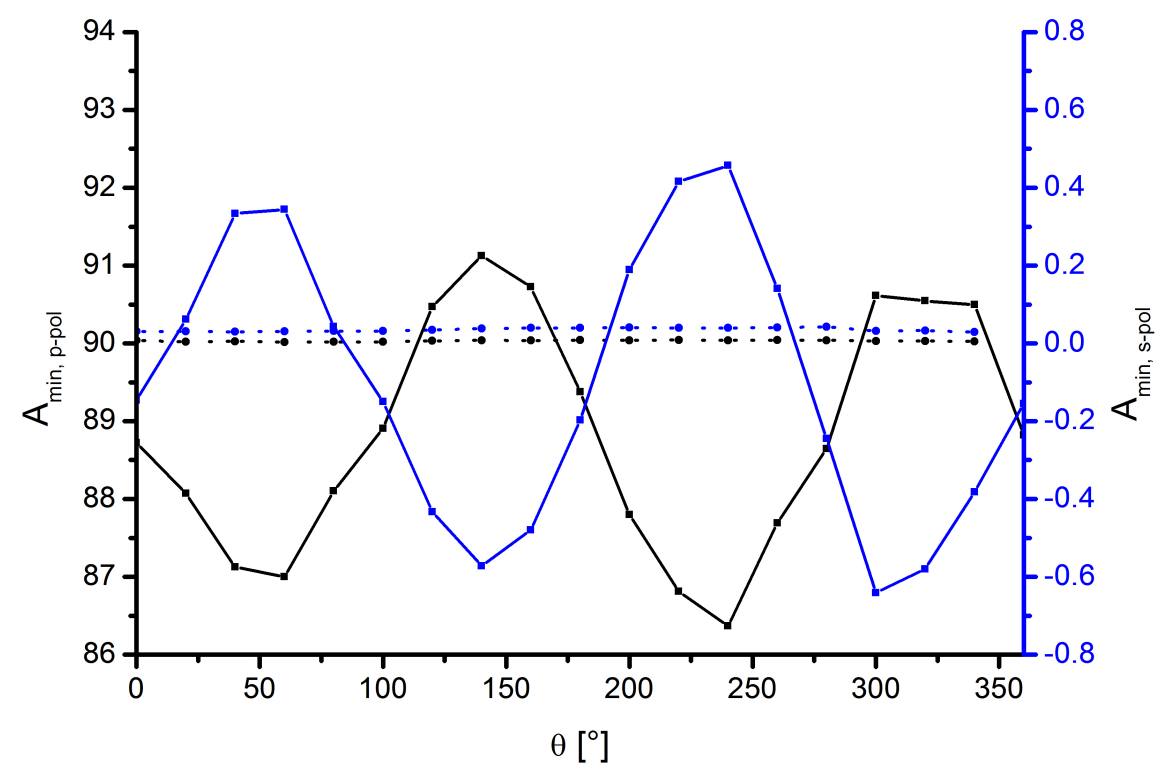

Abbildung 4.23 Theta Scan PET-Film (amorph) und PETFolie (kristallin).

Um den Brechungsindex des anisotropen Materials zu bestimmen, wurde nach einer Möglichkeit gesucht, die Brechungsindizes parallel und senkrecht zur Folienebene zu differenzieren. Da es sich bei der PET-Folie um ein transparentes Substrat handelt, sind prinzipiell die Bedingungen des Brewsterwinkels erfüllt, um anhand dessen ohne weitere Modellierung den Brechungsindex der Folie zu generieren (siehe Gleichung 2.14). Dazu wurde mit p-Polarisation (alle optische Komponenten) der AOI variiert und das Intensitätsminimum gesucht. Dieser Vorgang wurde für jeden Theta-Winkel wiederholt. Die berechneten Brechungsindizes sind in Abbildung 4.24 dargestellt. 


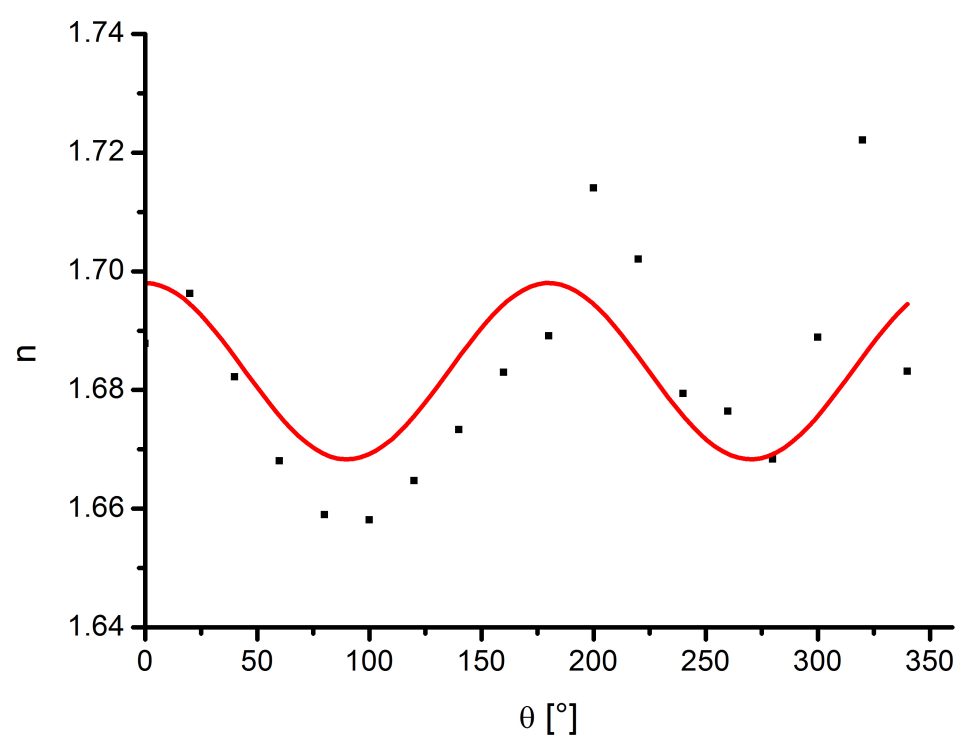

Abbildung 4.24 Brechungsindex der PET-Folie bei $658 \mathrm{~nm}$ in Abhängigkeit von Theta.

Nach Laskarakis und Logothetidis lässt sich der Brechungsindex von optisch uniaxialem PET mittels folgender Formel beschreiben [37]:

$$
\frac{1}{n^{2}(\theta)}=\frac{\sin ^{2} \theta}{n_{\|}^{2}}+\frac{\cos ^{2} \theta}{n_{\perp}^{2}}
$$

An den ermittelten Daten wurde analog die obige Funktion für uniaxiale Systeme angepasst. Der Wert für den ordinären und extraordinären Brechungsindex ist in Tabelle 4.7 gezeigt.

\begin{tabular}{|c|c|c|}
\hline$n_{\|}$ & $n_{\perp}$ & $\triangle n$ \\
\hline \hline $1.668 \pm 0.006$ & $1.698 \pm 0.006$ & 0.03 \\
\hline
\end{tabular}

Tabelle 4.7 Brechungsindex von uniaxial anisotroper PET-Folie.

Anhand der Messung lässt sich erkennen, dass die Ausrichtung der Probe eine wichtige Rolle bei der Messung spielt. Eine Änderung des Brechungsindex um 0.03 führt zu anderen Messergebnissen. Eine Ausrichtung der Probe gewährleistet die richtige Referenz als Substrat im Model zu berücksichtigen.

In dieser Arbeit wird die PET-Folie als uniaxial anisotropes Material behandelt, weil bei größerer Anisotropie des Substrates die Beschreibung mittels Jones-Matrix nicht ausreicht und klassische Ellipsometrie nicht genutzt werden kann. Bezüglich weiterer Untersuchungen anisotroper Substrate sei auf die Müller-Matrix-Ellipsometrie verwiesen [28]. 
Im nächsten Schritt wurde die PET-Folie spektroskopisch charakterisiert, um die Dispersion des Materials zu erhalten. Dazu wurde die PET-Folie bei einem Einfallswinkel von $50^{\circ}$ und $55^{\circ}$ spektroskopisch $(380-1000 \mathrm{~nm})$ vermessen. Die optischen Konstanten des transparenten Materials wurden durch ein Cauchy-Model beschrieben (siehe Kapitel 7).

Anschließend wurden verschiedene Dünnschichten von ITO, PEDOT:PSS, P3HT:PCBM und ALQ3 auf PET-Folie aufgebracht und spektroskopisch untersucht.

\section{ITO auf PET}

Indiumzinnoxid wird aufgrund seiner Transparenz wie auch seiner guten Leitfähigkeit als Elektrodenmaterial für organische Solarzellen eingesetzt. Auch die PET-Folie wurde mit diesem Material beschichtet. Es sollten sowohl die optischen Parameter als auch die Schichtdicke bestimmt werden. Dazu wurden spektroskopische Messungen bei einem $\mathrm{AOI}=50^{\circ}$ gemacht. Als Dispersionsmodel wurde eine Kombination aus einem Lorentz und einem Drude Term gewählt und an die experimentellen Daten des Wellenlängenspektrums angepasst. Zusätzlich wurde im Model eine Zwischenschicht - bestehend aus ITO und PET-Folie - angenommen, die mittels effective medium approximation nach Bruggeman (Kapitel 2.56) mit einem fraction guest factor von 0.5 beschrieben wurde. Sowohl die Schicht des ITOs als auch die Zwischenschicht wurde neben den Parametern von Lorentz und Drude mittels Fit bestimmt. In Abbildung 4.25 sind sowohl die experimentell bestimmten Delta- und Psi-Werte (Punkte) als auch die auf dem Model basierenden simulierten Wellenlängenspektren (Linien) von Delta (rot) und Psi (grün) gezeigt. Die Schichtdicke von ITO beträgt $94.2 \pm 0.8 \mathrm{~nm}$ und die Zwischenschicht $41.7 \pm 1.1 \mathrm{~nm}$. Das Vorhandensein der Zwischenschicht lässt sich durch die generelle Rauheit des Substrats erklären. Die erhaltene Dispersionfunktion für das ITO ist in der Abbildung 4.26 zu sehen. Die optischen Parameter stimmen mit denen überein, die in der Literatur zu finden sind $[40]$. 


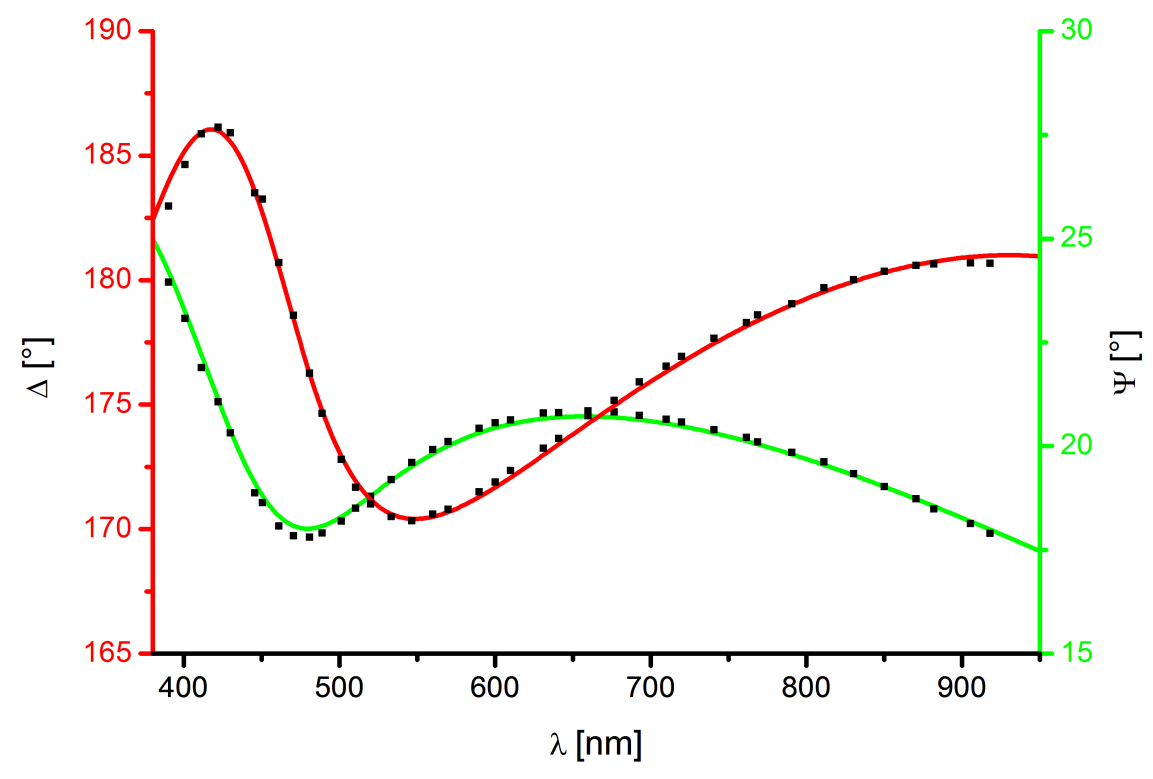

Abbildung 4.25 Delta/Psi-Wellenlängenspektrum ITO auf PET Folie.

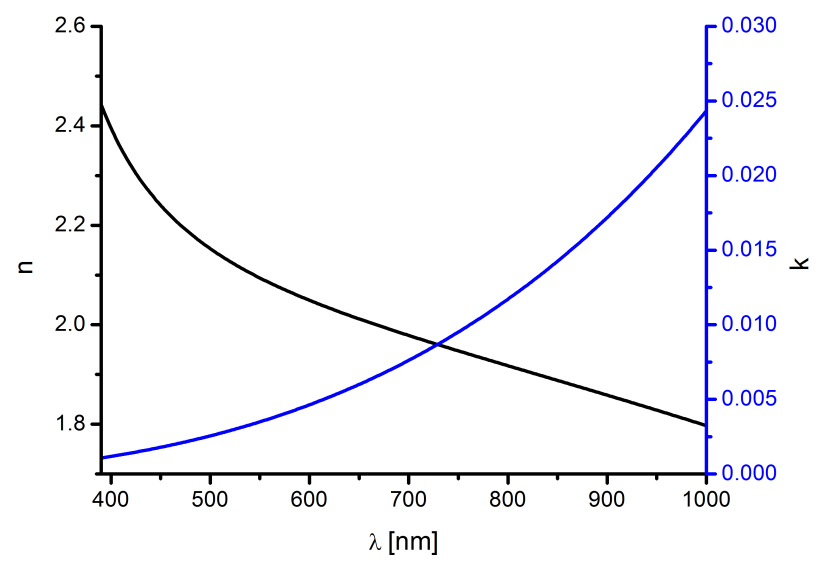

Abbildung 4.26 Dispersion ITO auf PET-Folie.

\section{PEDOT:PSS auf PET}

Als Elektrondonator bei organischen Solarzellen wird häufig eine Schicht von PEDOT:PSS aufgetragen. Das Dünnschichtsystem von PEDOT:PSS auf PET-Folie wurde mittels abbildender Ellipsometrie spektroskopisch - analog zum vorherigen Beispiel - charakterisiert (Abbildung 4.27). 


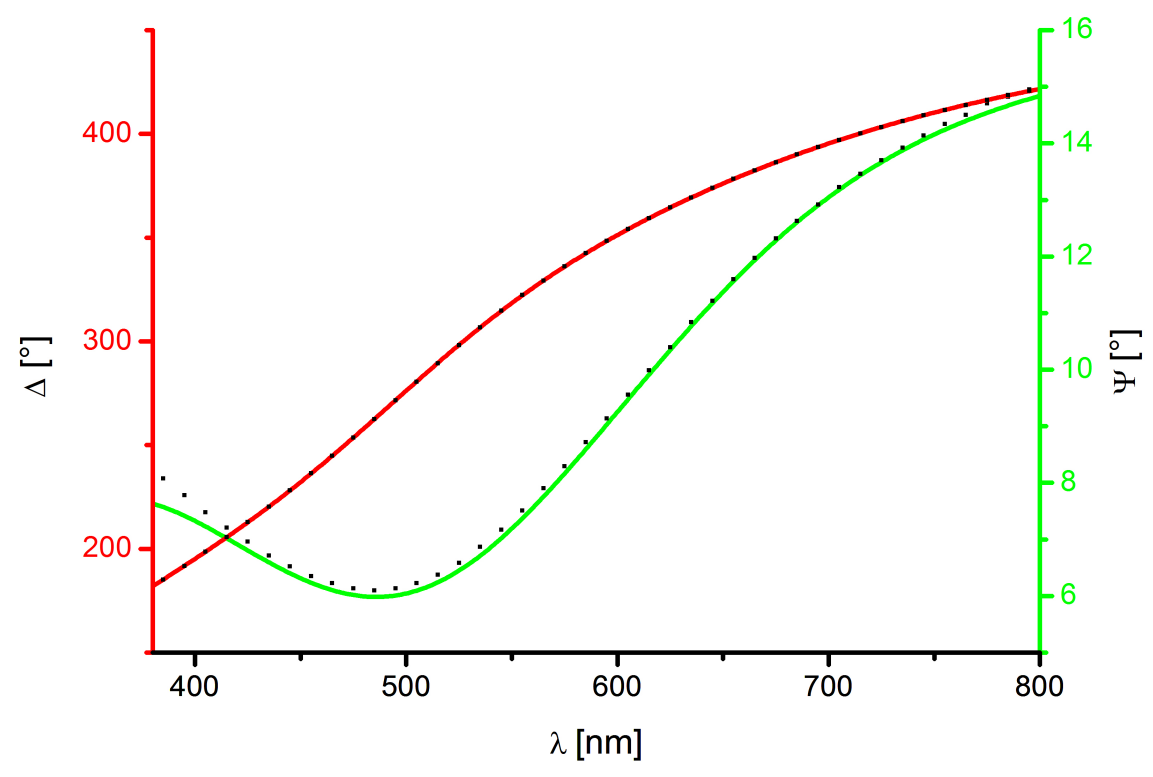

Abbildung 4.27 Delta/Psi-Wellenlängenspektrum PEDOT:PSS auf PET Folie.

Im optischen Modell wurde die Schicht des PEDOT:PSS als anisotrope Schicht behandelt. Dabei wurde zwischen den optischen Parametern senkrecht zum Substrat (z-Achse) und in der Probenebene (x- und y-Achse) differenziert. Bei beiden Richtungen wurde als Dispersionmodel eine Kombination aus einem Tauc-Lorentz-Term und einem Drude-Term verwendet. Unter der Annahme dass es sich um ein Einschichtsystem handelt, wurde nach der Schichtdicke und den Parametern der Dispersion gefittet. Die ermittelten optischen Parameter sind in Abbildung 4.28 dargestellt. Während die durchgezogenen Linien die Werte in der xy-Ebene repräsentieren, stehen die gestrichelten Linien für die Werte senkrecht zur Probenebene. 


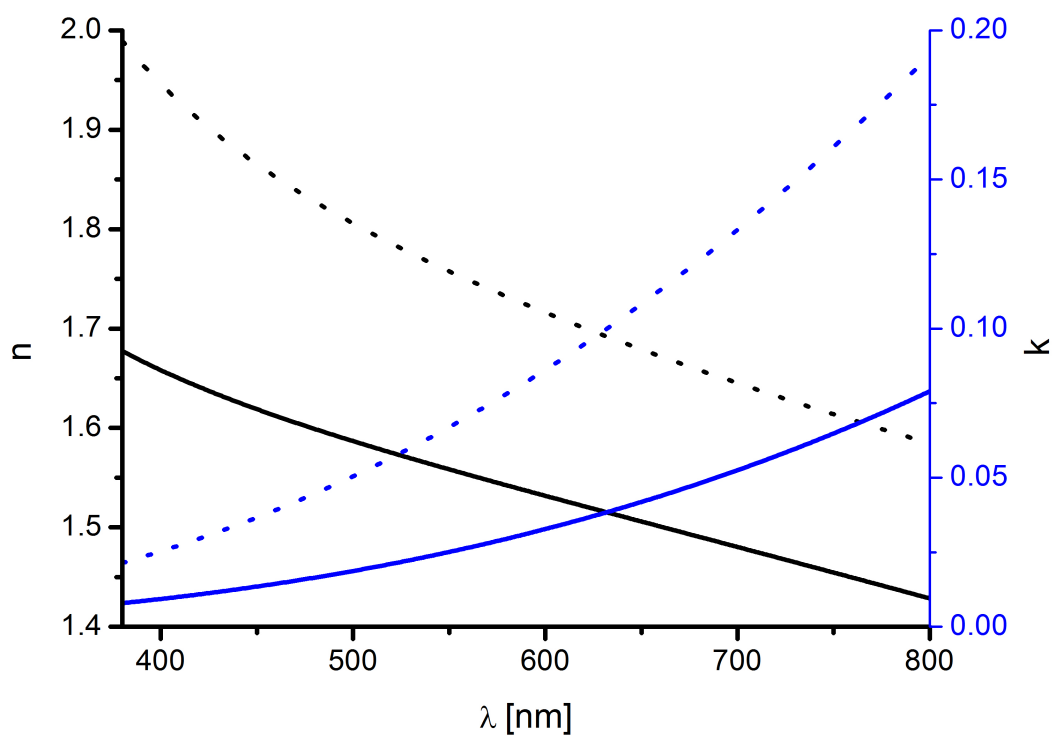

\begin{abstract}
Abbildung 4.28 Erhaltene Dispersion von PEDOT:PSS auf PET-Folie.
\end{abstract}

\title{
P3HT:PCBM auf PET
}

Als photoaktive Schicht wird sehr häufig im Bereich von plastic solar cells ein Gemisch aus P3HT als Elektronendonator und PCBM als Elektronenakzeptor eingesetzt. Bei den folgenden Proben wurde die photoaktive Schicht mittels Print-Prozess auf PET Folie aufgetragen. Bei dem Prozess wurde die Liniendichte beim Drucken variiert. Mittels ellipsometrischer Untersuchung sollte der Einfluss der Liniendichte $\left(120 \mu \mathrm{m}^{-1}, 140 \mu \mathrm{m}^{-1}, 160 \mu \mathrm{m}^{-1}\right.$ und $\left.210 \mu \mathrm{m}^{-1}\right)$ in Bezug auf die Schichtdicken sowie deren Varianz auf der PET-Folie untersucht werden. Dazu wurden die Proben bei einem festen AOI von $50^{\circ}$ spektroskopisch unter Verwendung des 10x Objektives und dem Beamcutter vermessen. Die ROIs (Anzahl $=34$, Größe $=15 \times 15 \mu \mathrm{m}$ ) wurden innerhalb des Schatten des Beamcutters in einer Linie angeordnet und ein Wellenlängenspektrum (2 Zonen) von 365-795 nm in $10 \mathrm{~nm}$ Intervallen aufgenommen. Die simultan aufgenommenen Wellenlängenspektren der ROIs wurden unter Zuhilfenahme des Ep4-Models mit Blick auf die Schichtdicke ausgewertet. Die Dispersion des Substrats PET wurde aus den vorherigen Messungen übernommen und die photoaktive Schicht aus P3HT und PCBM durch einen Tauc-Lorentz und zwei Lorentz Terme beschrieben. In Abbildung 4.29 sieht man exemplarisch das Delta/Psi-Spektrum von P3HT:PCBM auf PET-Folie von ROI 19 mit einer Liniendichte von 140. 


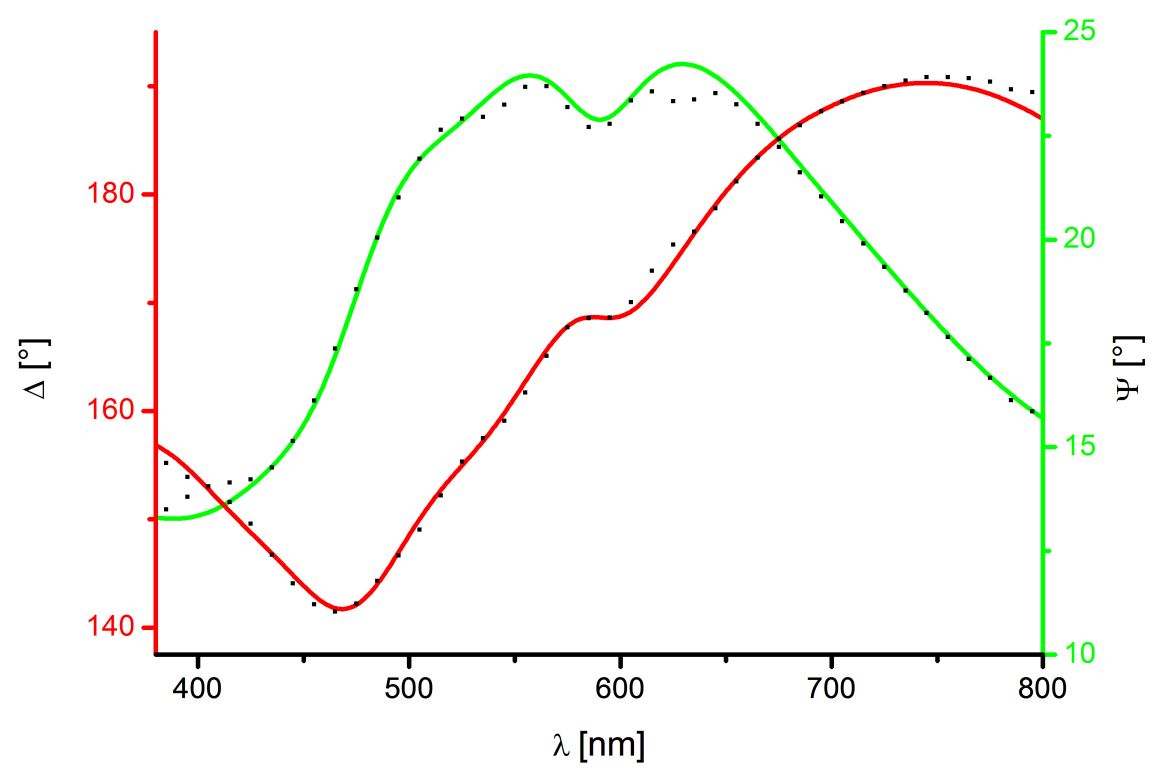

Abbildung $\quad 4.29$ Delta-/Psi-Wellenlängenspektrum von P3HT:PCBM auf PET Folie.

Die Dispersion (Abbildung 4.30) von P3HT:PCBM zeigt im visuellen Bereich (bei $500 \mathrm{~nm}$ ) eine starke Absorptionsbande. Diese entspricht in etwa dem Maximum der Strahlungsintensität des Sonnenlichtes. Beim Fitting-Prozess für die einzelnen ROIs wurden neben der Schichtdicke ebenfalls die Dispersionsparameter freigegeben. Die erhaltenen optischen Parameter zeigen marginale Abweichungen zu den hier dargestellten Werten. 


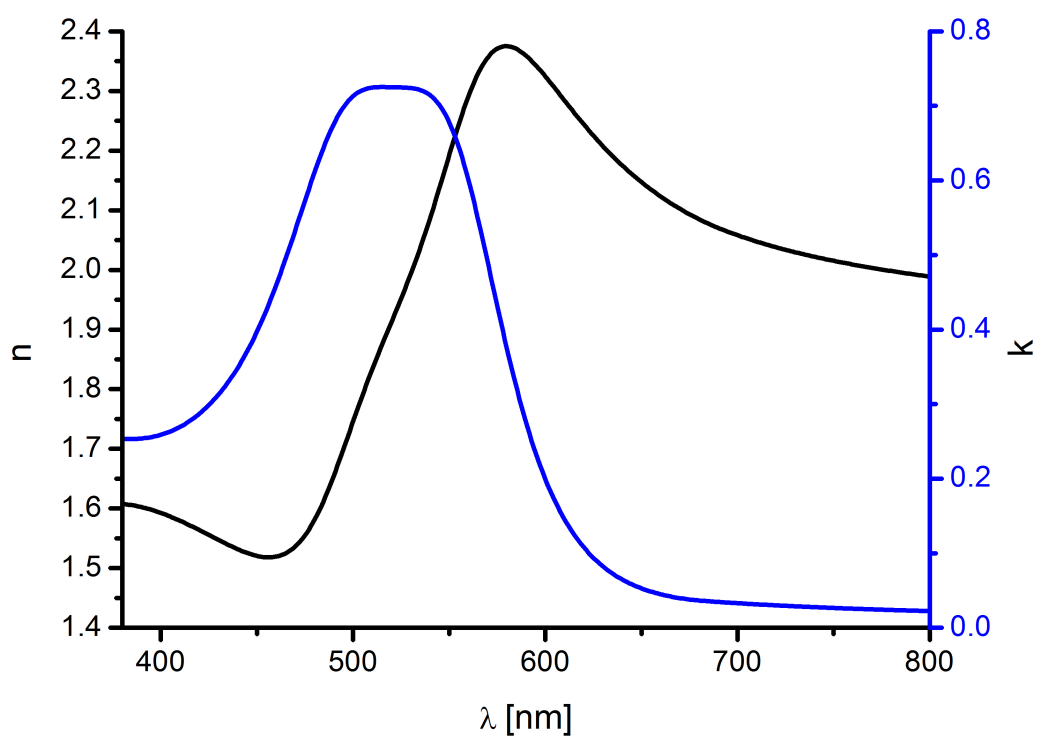

Abbildung 4.30 Ermittelte Dispersion von P3HT:PCBM auf PET-Folie.

Einen Ausschnitt der Delta-Map von der PET-Folie mit einer Schicht von P3HT:PCBM inklusive der Schichtdicke in Form eines Säulendiagramms zeigt Abbildung 4.31.

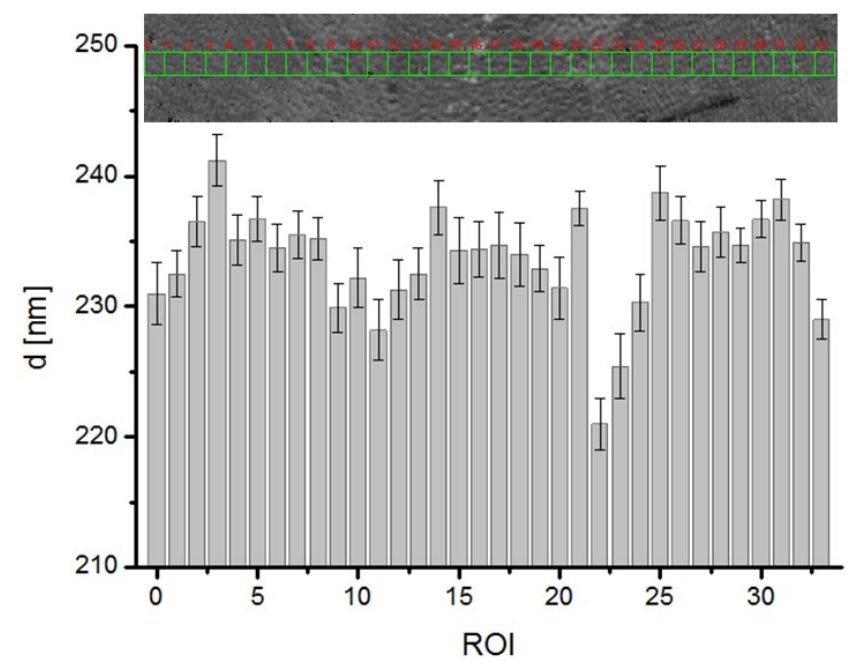

Abbildung 4.31 Delta-Map und Säulendiagramm der Schichtdicken von P3HT:PCBM des ROI-Profils.

Es ist deutlich zu erkennen, dass die Schichtdicke des P3HT:PCBM-Films über die verschiedenen ROIs variiert. In der Abblidung 4.31 sind zusätzlich die ermittelten 
Fehler der Schichtdicken in Form von Balken eingezeichnet. Der maximale Fehler bei dieser Probe liegt bei $2.5 \mathrm{~nm}$. Eine Übersicht der ROI-Profile der Proben mit variiender Liniendichte beim Druckprozess (Abbildung 4.32) zeigt eine deutliche Zunahme der Schichtdicke.

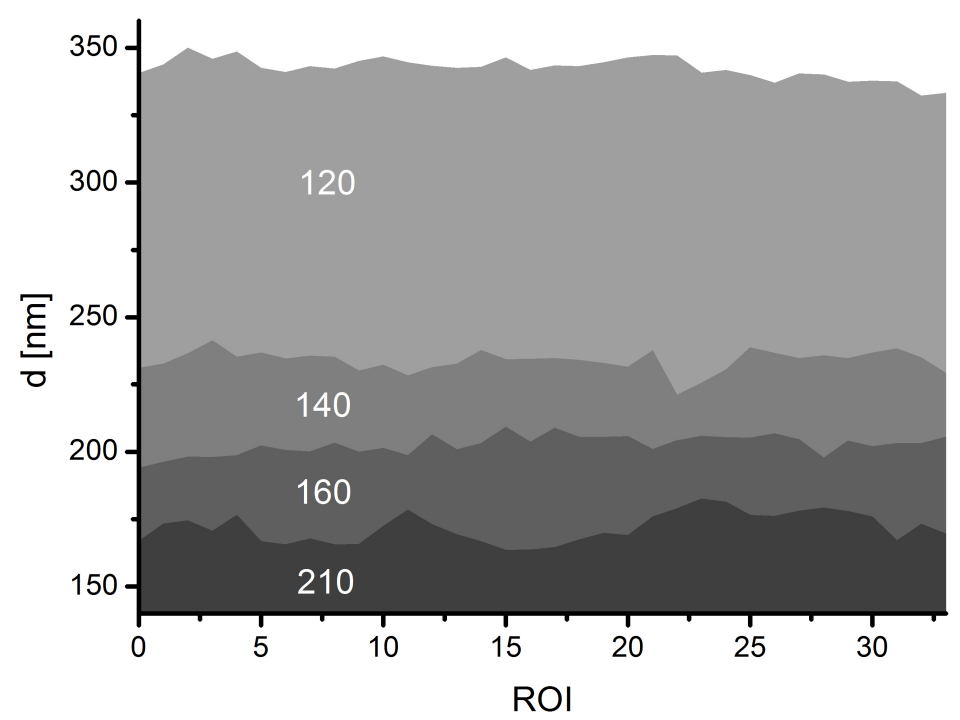

Abbildung 4.32 Übersicht über die Schichtdickenvariation von P3HT:PCBM auf PET-Folie innerhalb der ROI-Profile in Abhängikeit von der Liniendichte.

Eine Auftragung der gemittelten Schichtdicke sowie des relativen Fehlers in Abhängigkeit der Liniendichte ist in Abbildung 4.33 visualisiert. 


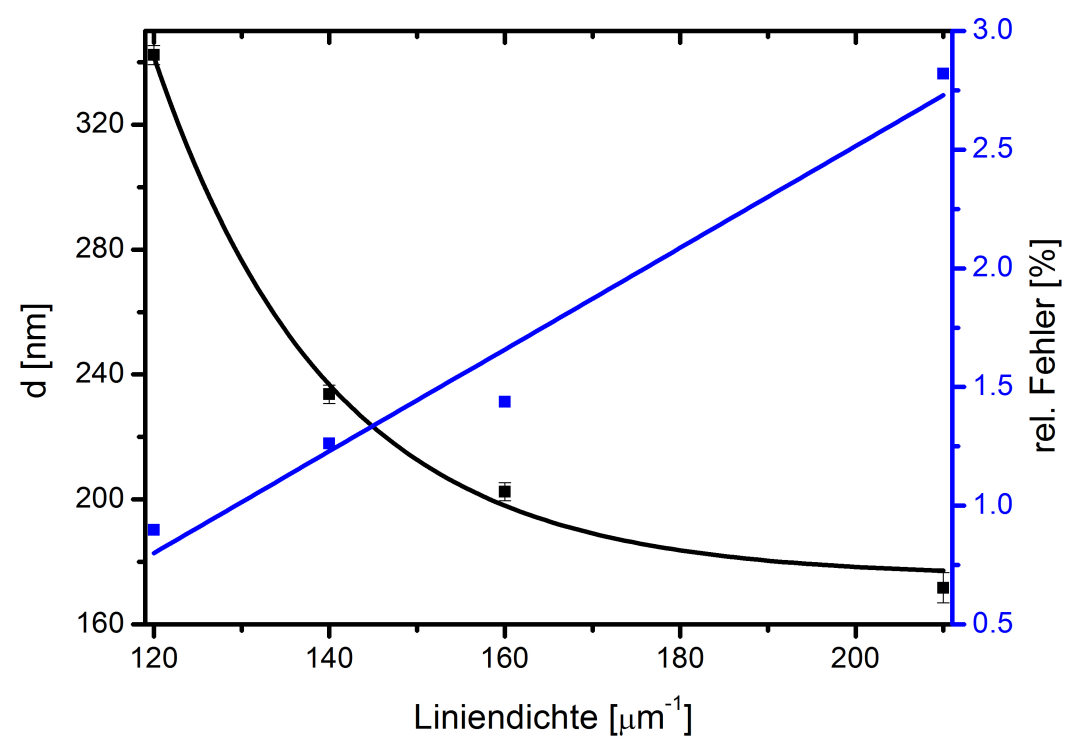

Abbildung 4.33 Übersicht der gemittelten Schichtdicke und des relativen Fehlers der P3HT:PCBM-Schicht auf PET-Folie in Abhängigkeit der Liniendichte.

Die Liniendichte beim Drucken ist definiert als Kehrwert der Linienabstände. Dies erklärt die Antiproportionalität des Zusammenhangs zwischen Liniendichte und Schichtdicke. An die gemittelten Schichtdickenwerte von P3HT:PCBM wurde ein exponentieller Fit (schwarze Linie) ${ }^{30}$ angelegt. Die Schichtdickenvariation innerhalb des ROI-Profils wird in Relation des Mittelwerts der jeweiligen Probe gezeigt. Dabei ergibt sich ein nahezu linearer Zuhammenhang ${ }^{31}$ zwischen der Schichtdickenvariation und der Liniendichte.

\section{AlQ3 auf PET}

Neben ITO wurde AlQ3 als organsiches Halbleitermaterial auf PET-Folie verwendet und spektroskopisch untersucht. Die ellipsometrischen Werte und das angepasste Spektrum werden in Abbildung 4.34 gezeigt.

\footnotetext{
${ }^{30} y=A \exp \left(-\frac{x}{t}\right)+y_{0}, \mathrm{~A}=65365,43 \mathrm{~nm}, \mathrm{t}=20.08 \mu \mathrm{m}^{-1}, \mathrm{y}_{0}=175.31 \mathrm{~nm}$

${ }^{31} y=a+b x, \mathrm{a}=-1,771 \%, \mathrm{~b}=0,021 \mu \mathrm{m} \%$
} 


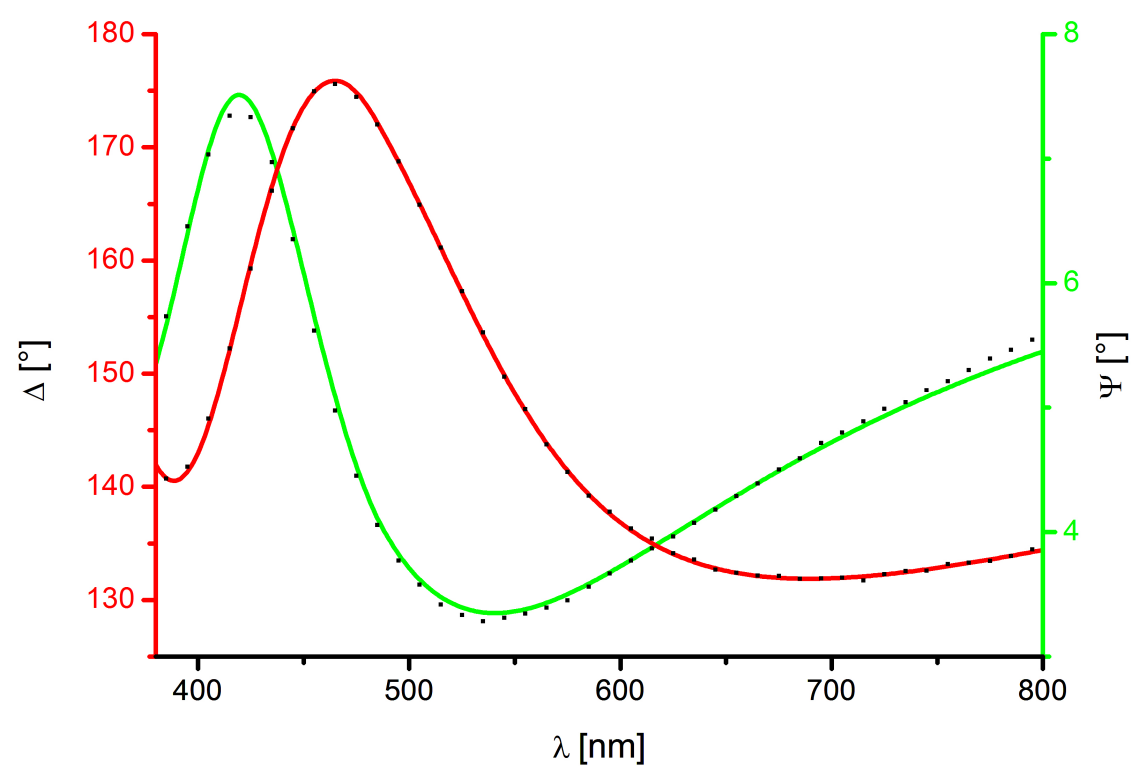

Abbildung 4.34 Delta-/Psi-Wellenlängenspektrum $\mathrm{AlQ}_{3}$ auf PET Folie.

Die optischen Eigenschaften des organsichen Halbleitermaterials wurden durch zwei Tauc-Lorentz-Terme, sowie durch einen Drude-Term beschrieben. Auch hier zeigt sich eine deutliche Verbesserung des Fits durch die Einbeziehung einer Zwischenschicht zwischen AlQ3 und dem Substrat. Wie auch beim ITO wurde die Zwischenschicht mittels effective medium approximation nach Bruggeman als Kombination beider Materialien ( $\mathrm{f}=0.5$ ) simuliert. Die aus dem Model berechnete Dispersion ist in Abbildung $4.35 \mathrm{zu}$ sehen. 


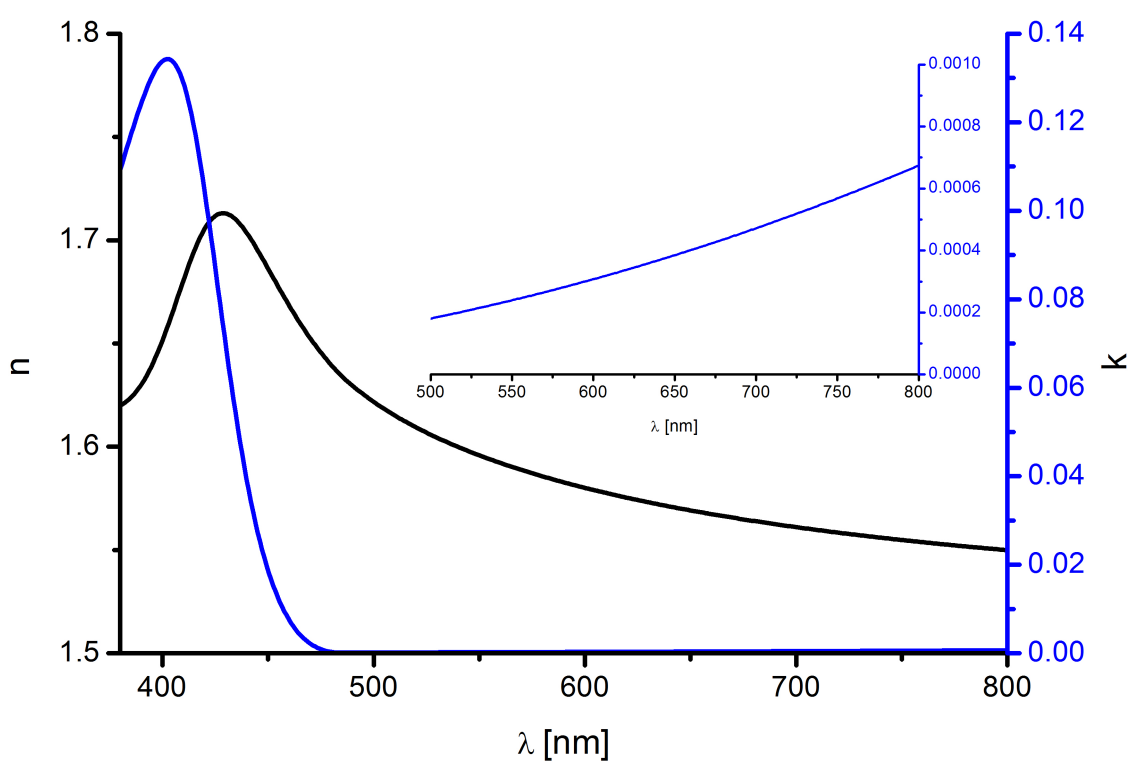

Abbildung 4.35 Dispersion von $\mathrm{AlQ}_{3}$ auf PET.

Im Verlauf des Absorptionskoeffizienten liegt bei $402 \mathrm{~nm}$ ein elektronischer Übergang im Form einer Absorptionsbande. Bei größeren Werten für die Wellenlänge zeigt das Material einen sehr geringen metallischen Charakter, der sich in einer Zunahme des Absoprtionskoeffizienten äußert und durch den Drude-Term wiedergespiegelt wird. Die durch lineare Interpolation bestimmte Bandlücke des Materials beträgt $450 \mathrm{~nm}$.

Vergleicht man die Werte mit denen aus der Literatur ist die Absoprtion des organischen Materials etwas geringer, wobei die charakteristische Lage der Absoprtionsbande und Bandlücke sehr gut übereinstimmen[15][17]. So hat beispielsweise das Lösungsmittels einen Einfluss auf die Lage der Bandlücke und kann somit ein Faktor der marginalen Unterschiede zur Literatur sein. 


\subsubsection{Glas}

Neben Silizium-Wafern werden - als kostengünstige Alternative - dünne Filme auf Glasoberflächen aufgetragen. Der Vorteil dieses Trägermaterials ist, dass es aufgrund seiner Transparenz, für UV/VIS-spektroskopische Untersuchungen genutzt werden kann, um das Filmmaterial hinsichtlich einer möglichen Absorption zu charakterisieren. In diesem Unterkapitel werden in Analogie zu den vorherigen Plastic Solar Zellen Materialien spektroskopisch untersucht. Glassubstrate zeigen in der Regel ein isotropes optisches Verhalten, was die Messungen und den Umgang mit diesem Material hinsichtlich der ellipsometrischen Untersuchung vereinfacht. Auch hier wird aufgrund von Rückseitenreflexionen ein Beamcutter eingesetzt.

Durch das Vermessen der Proben mit unterschiedlicher Schichtdicke war es möglich, für beide Materialien einen linearen Zusammenhang zwischen der Schichtdicke und charakteristischen IR-Banden zu beobachten.

Die Messungen wurden bei einem Einfallswinkel von $50^{\circ}$ und im Wellenlängenbereich von 380-1000 nm durchgeführt. Durch die Nutzung des Filterrads wurden feste Wellenlängen $($ Anzahl $=43)$ vermessen, die unterschiedliche Intervalle besaßen. Als Objektiv wurde das 10x benutzt.

\section{PEDOT:PSS auf Glas}

Die gemessenen Delta-/Psi-Wellenlängenspektren der Proben bei verschiedener Rotationsgeschwindigkeit sind in Abbildung 4.36 gezeigt. Bereits anhand des Verlaufs in Delta ist deutlich eine Abnahme der Schichtdicke bei Erhöhung der Rotationsgeschwindigkeit zu erkennen.
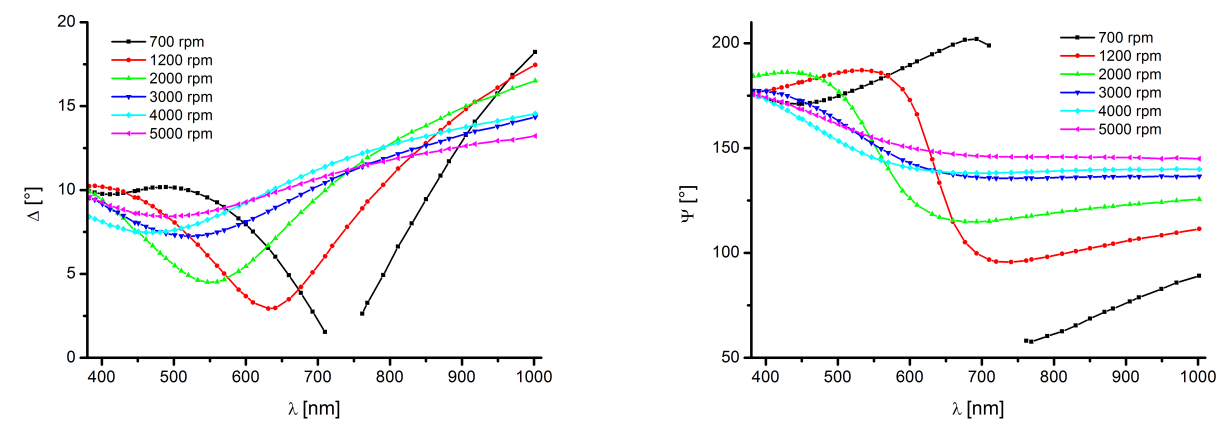

Abbildung 4.36 Delta-/Psi-Wellenlängenspektren von PEDOT:PSS auf Glas bei variiender Rotationsgeschwindigkeit.

Das optische Verhalten des Schichtmaterials wurde durch eine Kombiantion aus einem Lorentz- und einem Drude Term beschrieben. Die mittels Ep4-Model generierten Schichtdicken in Abhängigkeit von der Rotationsgeschwindigkeit, sowie deren absoluter Fehler sind in Abbildung 4.37 zu sehen. 


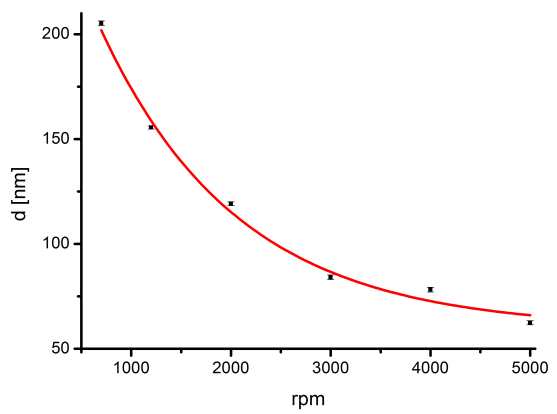

\section{Abbildung 4.37 Schichtdicke versus Rotationsgeschwindigkeit PEDOT:PSS.}

Zusätzlich wurden die Proben mittels ATR-FTIR-Spektroskopie untersucht und die charaketristschen IR-Banden (Abbildung 4.38) des Filmmaterials den jeweiligen Schwingungen zugeordnet. Neben den symmetrischen $\left(2872 \mathrm{~cm}^{-1}\right)$ und asymmetrischen $\left(2925 \mathrm{~cm}^{-1}\right)$ CH-Streckschwingungen ist besonders der Fingerprintbereich charakteristisch für das Material. Neben den asymmetrischen $\mathrm{C}=\mathrm{C}$-Strechschwinungen bei $1510 \mathrm{~cm}^{-1}$ und $1305 \mathrm{~cm}^{-1}$, den Schwinungen $1188 \mathrm{~cm}^{-1}, 1127 \mathrm{~cm}^{-1}$ und $1079 \mathrm{~cm}^{-1}$ für die C-O Bindung der ethylendioxy-Einheit findet man zusätzlich einen Peak bei $1006 \mathrm{~cm}^{-1}$ für die S-O Bindung im $\mathrm{SO}_{3}{ }^{-}$Molekül. Die restlichen Banden bei 969 $\mathrm{cm}^{-1}, 923 \mathrm{~cm}^{-1}, 835 \mathrm{~cm}^{-1}$ und $685 \mathrm{~cm}^{-1}$ sind charakteristische Peaks für die ThiophenEinheit.

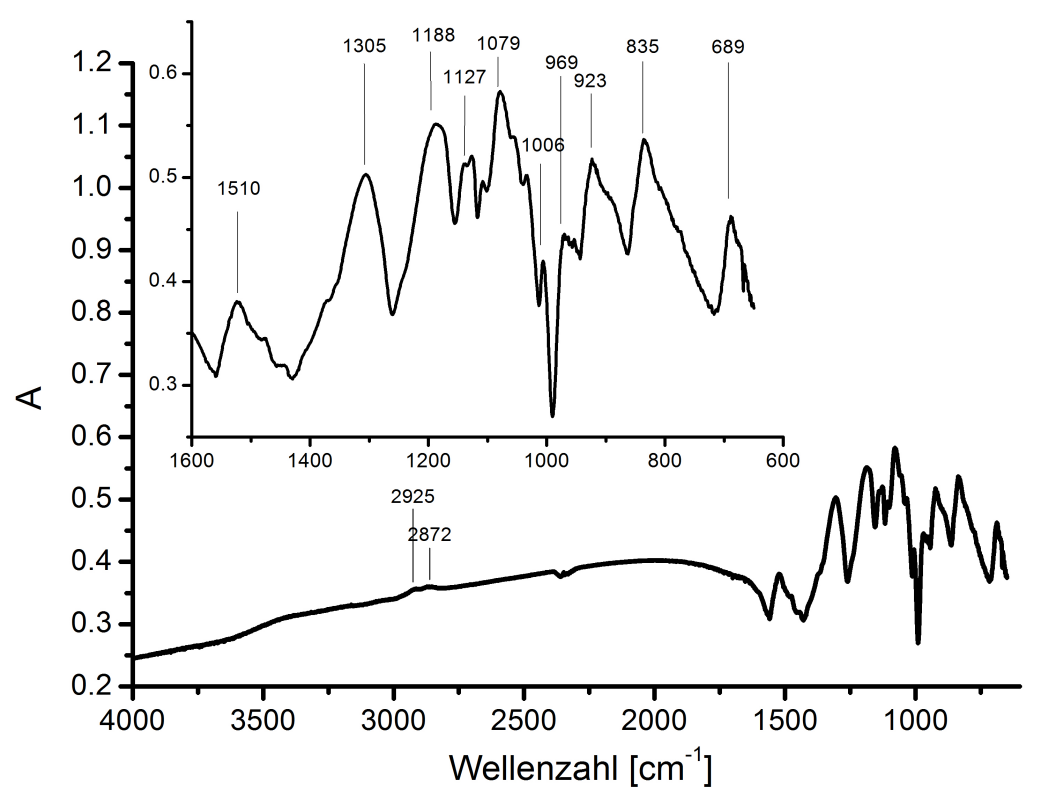

\footnotetext{
Abbildung 4.38 ATR-FTIR Spektrum von PEDOT:PSS auf Glas.
}

Die Fläche unter dem IR-Spektrum im Fingerprintbereich zeigt eine lineare Abhängigkeit (Abbildung 4.38). Dazu wurde die Fläche unterhalb des Spektrums gegen 
die Schichtdicke der ellipsometrischen Messungen aufgetragen. Mittels dieser Kalibrierung kann man die Schichtdicke von PEDOT:PSS mittels ATR-IR-Messungen bestimmt werden.
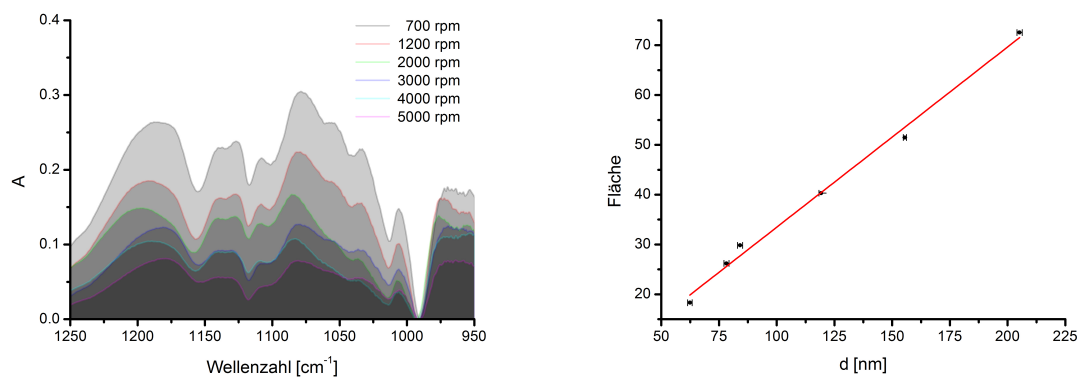

Abbildung 4.39 (Links) Fingerprint Bereich des ATR-FTIRSpektrums von PEDOT:PSS Filmen bei variierender Roataionsgeschwindigkeit des Herstellunsgprozesses sowie (rechts) lineare Korrelation zwischen Schichtdicke und Fläche der Absorptionsbande. 


\title{
P3HT:PCBM auf Glas
}

Analog zu den vorherigen Messungen wurde auch Proben mit variierender Schichtdicke von P3HT:PCBM auf Glas spektroskopisch vermessen (Abbildung 4.40).
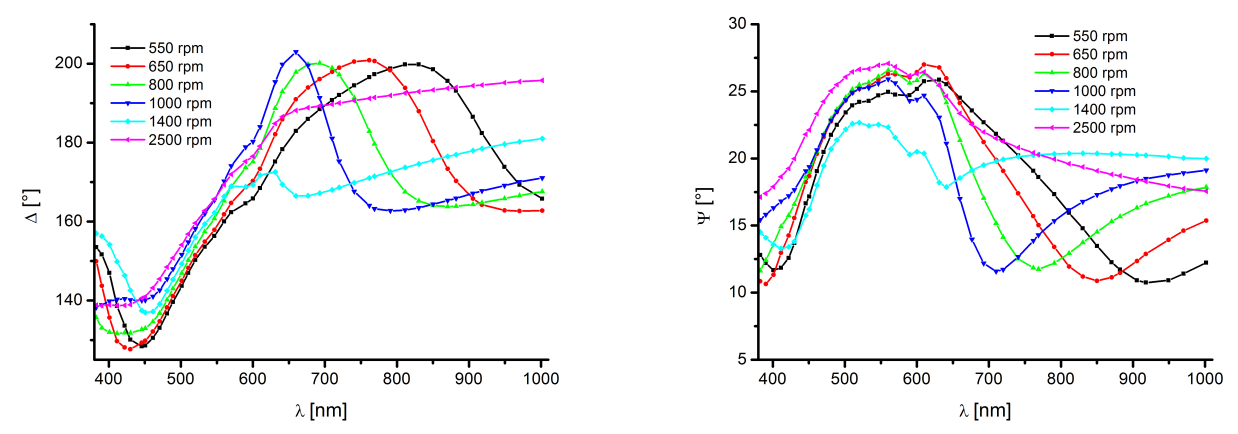

\begin{abstract}
Abbildung
4.40 Delta-/Psi-Wellenlängenspektren von P3HT:PCBM auf Glas bei variierender Rotationsgeschwindigkeit.
\end{abstract}

Neben den optischen Parametern wurde ebenfalls die Schichtdicke des photoaktiven Schicht bestimmt, die in Abbildung 4.41 gegen die Rotationsgeschwindigkeit aufgetragen wurde. Dabei zeigt die durch Spin Coating hergestellte Schichticke wie PEDOT:PSS eine Abnahme bei steigender Rotationsgeschwindigkeit.

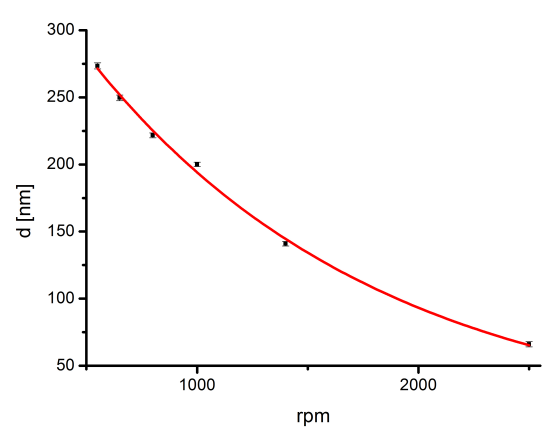

\begin{abstract}
Abbildung 4.41 Schichtdicke versus Rotationsgeschwindigkeit von P3HT:PCBM.
\end{abstract}

Zusätzlich wurden die Proben mittels ATR-FTIR-Spektroskopie untersucht und die charaketristschen IR-Banden (Abbildung 4.42) des Filmmaterials den jeweiligen Schwingungen zugeordnet. Neben den symmetrischen $\left(2858 \mathrm{~cm}^{-1}\right)$ und asymmetrischen $\left(2925 \mathrm{~cm}^{-1}\right) \mathrm{CH}-$ Streckschwingungen ist besonders der Fingerprintbereich charakteristisch für das Material. Neben den asymmetrischen $\mathrm{C}=\mathrm{C}-\mathrm{Strechschwinungen}$ bei $1485 \mathrm{~cm}^{-1}$, der Thiopheneinheit, findet man zusätzlich einen starken Peak bei $17396 \mathrm{~cm}^{-1}$ für die $\mathrm{C}=\mathrm{O}$ Bindung der Esterfunktion des PCBM-Moleküls. 


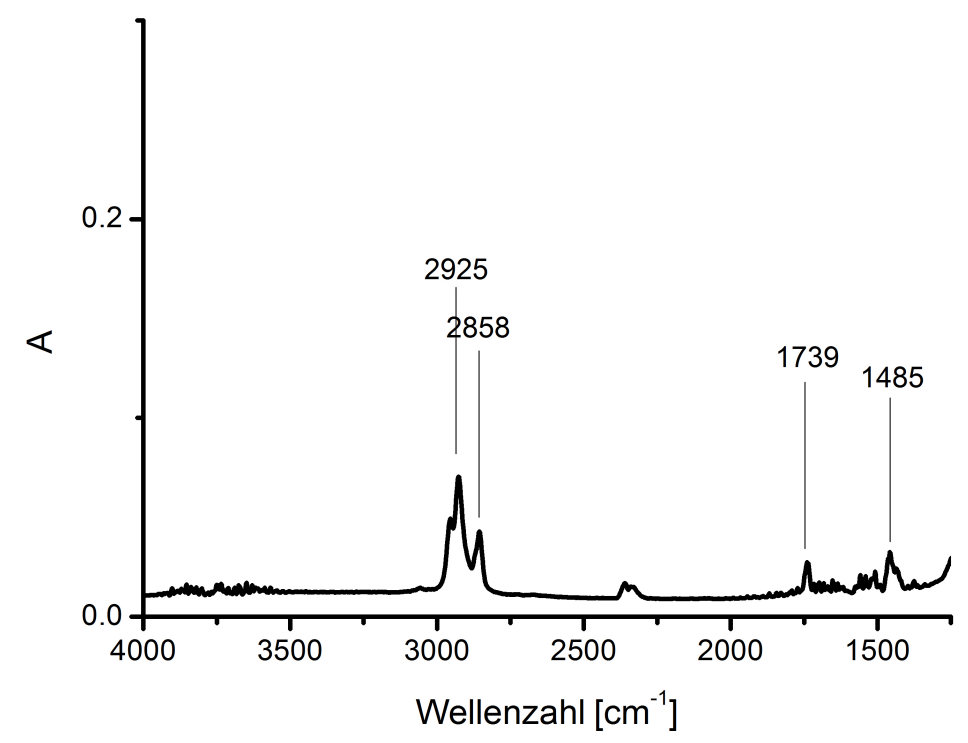

Abbildung 4.42 ATR-FTIR Spektrum von P3HT:PCBM auf Glass.

Die CH-Banden im IR-Spektrum wurden ebenfalls bezüglich dem Einfluss der Schichtdicke betrachtet und durch eine Auftragung (Abbildung 4.43) ein linearer Zusammenhang für die ATR-Messungen bestätigt.
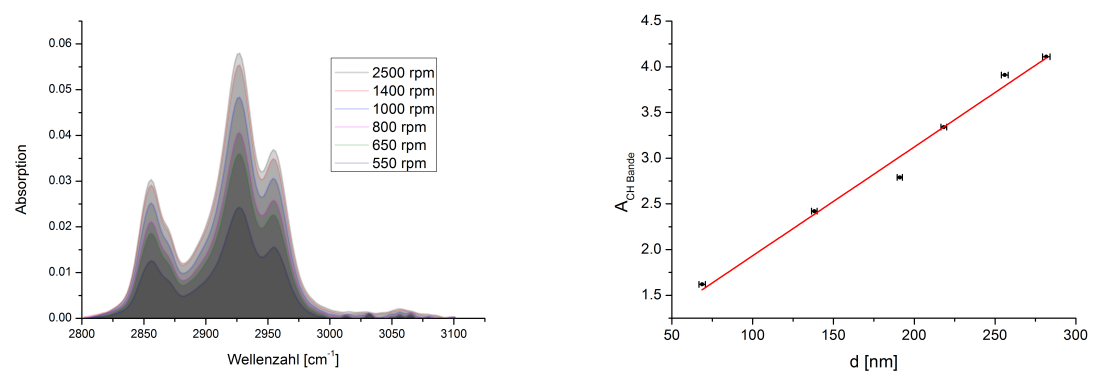

Abbildung 4.43 (Links) Fingerprint Bereich des ATR-FTIRSpektrums von P3HT:PCBM Filmen mit variierender Roataionsgeschwindigkeit beim Herstellunsgprozess sowie (rechts) lineare Korrelation zwischen Schichtdicke und Fläche der CHAbsorptionsbande. 


\subsubsection{Kontaktwinkelmessungen}

Wie bereits im Methodenteil erwähnt, wurden an planaren unstrukturierten Oberflächen Kontaktwinkelmessungen mit einem abbildenden Ellipsometer durchgeführt. Eine Übersicht der Ergebnisse ist in Tabelle $4.8 \mathrm{zu}$ sehen. Es wurden bekannte Materialien genutzt, um Daten zu generieren, die mit der Literatur verglichen werden können. Diese Proben sind außerdem unstrukturiert und eben. In der Tabelle 4.8 wird eine exemplarische Aufnahme und daneben wird der über die Messungen gemittelte Kontaktwinkel sowie die Standardabweichung gezeigt.

Die Materialien wurden nach zunehmendem Kontaktwinkel sortiert. Als super hydrophobes Material zeigt sich dabei das Flourcarbon. Die polymeren Materialien (PMMA, PS, PET) besitzen zwar hydrophoben Charakter, die Kontaktwinkel sind jedoch im Vergleich zur Literatur deutlich größer. Ein solcher Effekt kann durch nicht ideal ebene Schichten hervorgerufen werden. Denn eine Erhöhung der Rauigkeit kann zu einer Erhöhung des Kontaktwinkels führen. Es ist deutlich zu erkennen, dass das unbehandelte Silizium-Substrat mit nativer $\mathrm{SiO}_{2}$-Schicht deutlich weniger hydrophil ist als nach der Hydrophilisierung. Zwar lässt sich der Kontaktwinkel beim hydrophiler $\mathrm{SiO}_{2}$-Oberfläche nur schlecht bestimmen, weil nicht der ganze Wassertropfen zusehen ist, jedoch sollte sich dieser Effekt durch Minimierung des Tropfenvolumens beseitigen lassen. Es kann deutlich gezeigt werden, dass ein abbildendes Ellipsometer, neben der Verwendung als Polarisationsmikroskop, dazu genutzt werden kann Kontaktwinkelmessungen durchzuführen. Zusätzlich bietet die Verwendung eines abbildenden Ellipsometers die Chance, die Stellen zuvor ellipsometrisch zu untersuchen. Dabei könnten Einflüsse, wie Mikrostrukturen oder Rauigkeit, auf den Kontaktwinkel untersucht werden. 


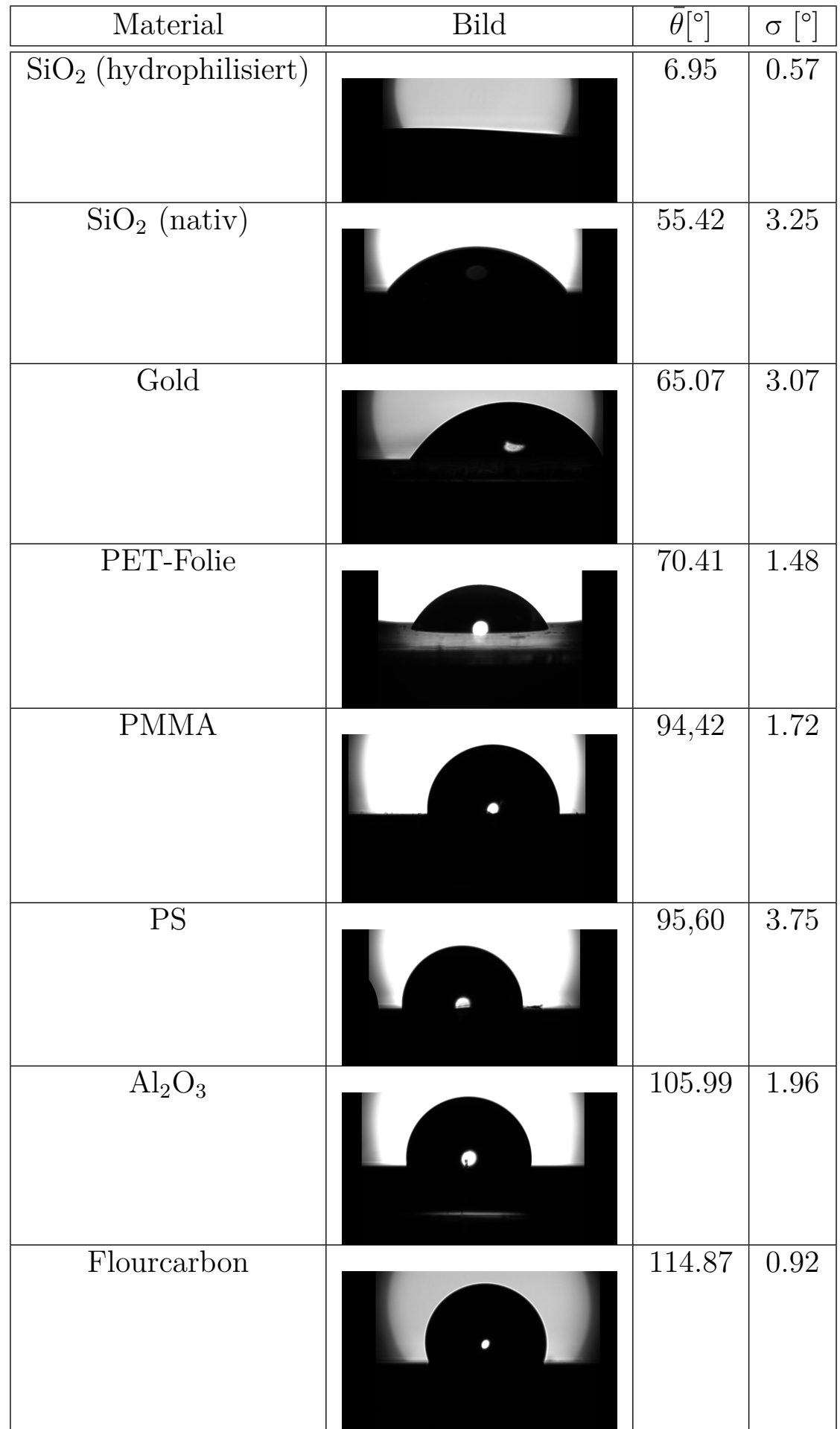

Tabelle 4.8 Kontaktwinkels von Wasser auf verschiedenen Materialien. 


\subsection{Spektroskopie}

$\mathrm{Zu}$ Beginn der Anfertigung dieser Arbeit stand dem Anwender für spektroskopische Messungen das Filterrad im Bereich von 360-1000 nm zur Verfügung. Dabei konnten Schichtdicken von bis zu $1 \mu \mathrm{m}$ spektroskopisch bestimmt werden. Neben der Erweiterung des spektroskopischen Bereichs wie UV bzw. NIR wurde gleichzeitig das Filterrad durch einen Monochromator mit durchstimmbaren Wellenlängen ersetzt.

\subsubsection{Monochromator}

Dabei wird als dispersives Element ein Reflektionsgitter verwendet, das neben einer Aufspaltung des polychromatischen Lichtes zu einer Bündelung des divergenten Strahles führt. Die Xenon-Lampe wurde durch eine gepulste Xenon-Lampe ausgetauscht, die deutlich mehr Intensität liefert. Im Vergleich zum Filterrad, der mit Transmissionsfiltern der jeweiligen Wellenlänge bestückt ist, hat der Monochromator 3 entscheidende Vorteile. Die Durchstimmbarkeit der Wellenlänge, die geringere Bandbreite und der geringere bzw. nicht auftretende Verschleiß. Die Transmissionsfilter beim Filterrad zeigten häufig nach längerem Gebrauch Zersetzungserscheinungen und mussten ausgetauscht werden. Beim Monochromator, der dispersiv arbeitet, bleibt ein solcher Zersetzungsprozess aus. Aufgrund der Anzahl an verfügbaren Datenpunkten beim Filterrad war die zu bestimmbare Schichtdicke bei spektroskopischen Messungen auf $1 \mu \mathrm{m}$ begrenzt. Mit dem Monochromator können nun jedoch deutlich dickere Schichtdicken spektroskopisch untersucht werden (bis $5 \mu \mathrm{m}$ wurden realisiert). Bei der Vermessung der maximalen Schichtdicke und anderen mikrometerdicken Schichten fiel auf, dass die im Ep4 Model simulierten Daten nicht zu den gemessenen Daten passten. Grund war die nicht Einbeziehung der Bandbreite in das simulierte Spektrum von Delta und Psi. Bei flachen Verläufen spielt die Bandbreite eine untergeordnete Rolle und fällt erst bei starken Oszillationen im Spektrum auf. Der Effekt der Bandbreite wurde in das Ep4-Model erfolgreich implementiert und getestet. Im nächsten Schritt werden den aufgenommenen Daten direkt die jeweilige Bandbreite angehangen und automatisch im Model berücksichtigt. Denn über den spektralen Bereich kann je nach Lichtquelle (durchstimmbarer Laser) und Gittertyp die Bandbreite $\Delta \lambda_{\mathrm{sp}}$ variieren. 

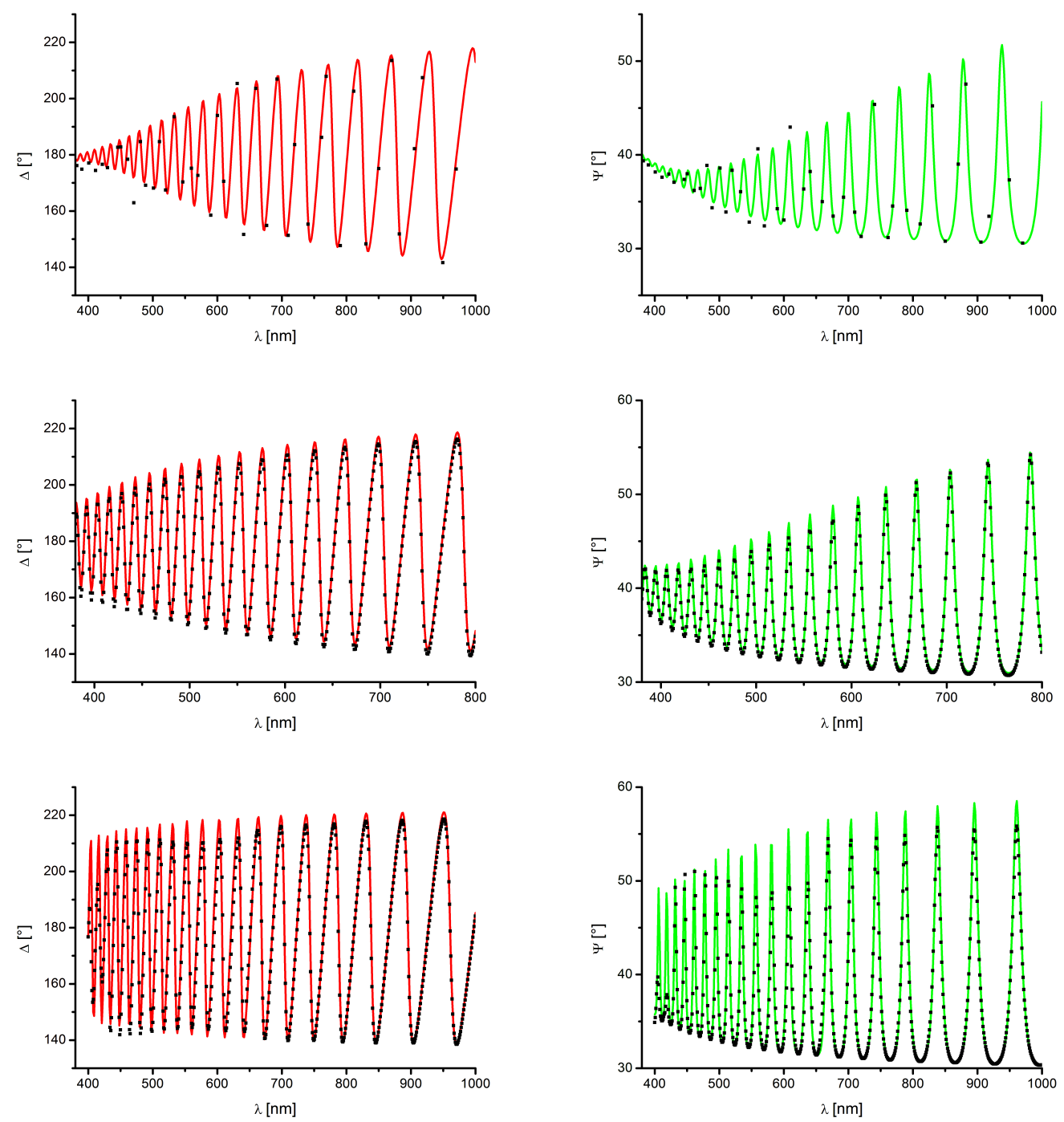

\begin{abstract}
Abbildung 4.44 Spektroskopische Ellipsometrie von $\mathrm{SiO}_{2}$ auf Si (100) unter Verwendung verschiedener Lichtquellen (oben: Filterrad $\left(\Delta \lambda_{\mathrm{sp}}=10 \mathrm{~nm}\right)$, mitte: Monochromator $\left(\Delta \lambda_{\mathrm{sp}}=5 \mathrm{~nm}\right)$, unten: durchstimmbarer Laser $\left(\Delta \lambda_{\mathrm{sp}}=1.8 \mathrm{~nm}\right)$.
\end{abstract}

Um den Effekt der Bandbreite bezüglich spektroskopsicher Messungen dicker transparenter Filme zu zeigen, wurde eine Probe mit etwa $5 \mu \mathrm{m}$ an $\mathrm{SiO}_{2}$ auf $\mathrm{Si}$ (100) ellipsometrisch vermessen. Dabei werden in jeder Abbildung sowohl die experimentellen Daten (Punkte) als auch das simulierte Spektrum aus dem Ep4-Model (Linie) gezeigt. Die optischen Parameter für $\mathrm{SiO}_{2}$ und $\mathrm{Si}(100)$ wurden aus der bestehenden Datenbank genutzt und lediglich nach der Schichtdicke des $\mathrm{SiO}_{2}$ gefittet. Bei den simulierten Daten wurde zusätzlich die Bandbreite berücksichtigt. Es wurde jeweils bei einem AOI $=50^{\circ}$ und unter Verwendung verschiedener Lichtquellen bzw. dispersiver Elemente gemessen. Oben sind die Daten dargestellt, die bei einer spektroskopischen Messung mittels Filterrad (Transmissionsfilter, $\Delta \lambda_{\mathrm{sp}}=10 \mathrm{~nm}$ ) entstanden. Es ist deutlich zu erkennen, dass es, aufgrund der großen Bandbreite, zu 
einer starken Stauchung der Oszillationen kommt. Der Effekt nimmt in Richtung des UV-Bereichs zu, weil die Breite der Oszillationen im Spektrum schmaler werden.

Das mit dem Monochromator (Transmissionsgitter, $\Delta \lambda_{\mathrm{sp}}=5 \mathrm{~nm}$ ) erhaltene Wellenlängenspektrum wird in der Mitte dargestellt. Der Effekt der Stauchung ist weniger stark ausgeprägt im Vergleich zum Filterrad. Als letzte Variante wurde die gleiche Messung mit einem durchstimmbaren Laser als Lichtquelle gemessen. Die sehr geringe spektrale Bandbreite $\Delta \lambda_{\mathrm{sp}}=1.8 \mathrm{~nm}$ führt erst im UV Beriech zu einer minimalen Stauchung des Wellenlängensprektums. Neben dem Effekt der Bandbreite ist deutlich zu erkennen das beim Filterrad lediglich 44 Datenpunkte mit Intervallen von über $10 \mathrm{~nm}$ generiert. Im Vergleich dazu wurde bei den anderen beiden Messungen Intervalle von $1 \mathrm{~nm}$ ausgewählt. Als Schichtdicke ergab sich bei der Messung mit dem Filterrad ein Wert von $5515.6 \pm 1.9 \mathrm{~nm}$ für die $\mathrm{SiO}_{2}$-Schicht, was gegenüber der Herstellerangabe einer Abweichung von $10 \%$ entsprechen würde. Bei den anderen beiden Messungen resultieren Schichtdicken von $5262.6 \pm 0.3 \mathrm{~nm}$ für den durchstimmbaren Laser und $5261.3 \pm 0.3 \mathrm{~nm}$ beim Monochromator. Die Abweichung der Schichtdicke stimmt mit dem vom Hersteller angegebenen Wert (5\%) überein.
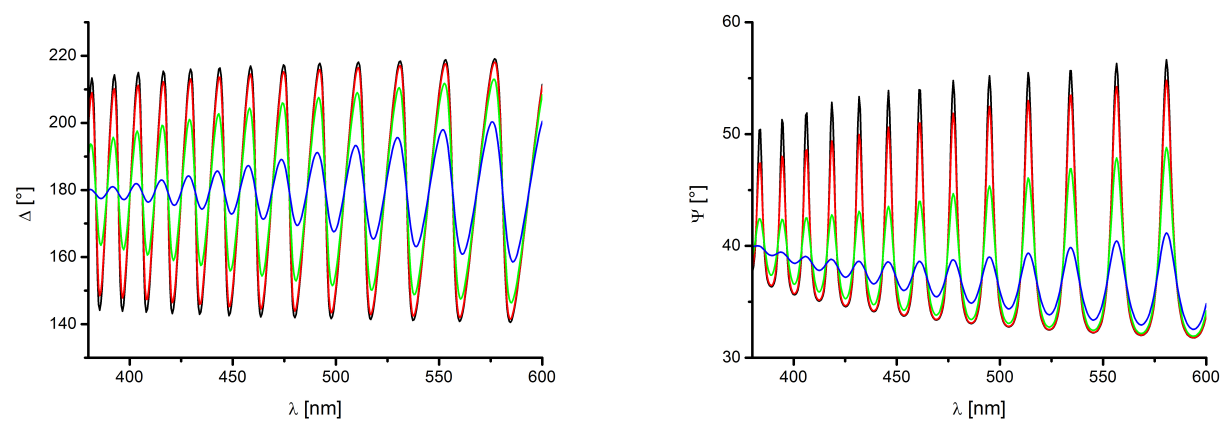

\begin{abstract}
Abbildung 4.45 Bandbreiten Effekt bei der Simulation von Delta/Psi-Wellenlängenspektren am Beispiel von $\mathrm{SiO}_{2}(\mathrm{~d}=5 \mathrm{~m})$ auf Si (100).
\end{abstract}

Anhand der Messungen wurde gezeigt, dass Schichtdicken über $5 \mu \mathrm{m}$ mit Hilfe von durchstimmbaren Lichtquellen Monochromator bzw. Laser mit geringerer Bandbreite als die Transmissionsfilter spektroskopisch eindeutig bestimmt werden können. Der Effekt der Bandbreite ist zur Verdeutlichung zusätzlich im Bereich von 380$600 \mathrm{~nm}$ in Abbildung 4.45 dargestellt. Die simulierten Daten basieren auf dem Probenmodel mit einer $5 \mu \mathrm{m}$ dicken $\mathrm{SiO}_{2}$-Schicht auf Silizium-Substrat und unter der Annahme eine Einfallswinkels von $50^{\circ}$. Es wird als Vergleich ein Spektrum ohne Einbeziehung der spektralen Bandbreite (schwarz) und mit spektraler Bandbreite $(2 \mathrm{~nm}=$ rot, $5 \mathrm{~nm}=$ grün, $10 \mathrm{~nm}=$ blau $)$ gezeigt.

Neben der Erhöhung der maximal bestimmbaren Schichtdicke können aufgrund der Durchstimmbarkeit der Wellenlänge die optischen Konstanten von Materialien genauer bestimmt werden, wie beispielsweise die Bandlücke von Halbleitern oder Absorptionspeaks innerhalb des gemessen Wellenlängenbereichs. 

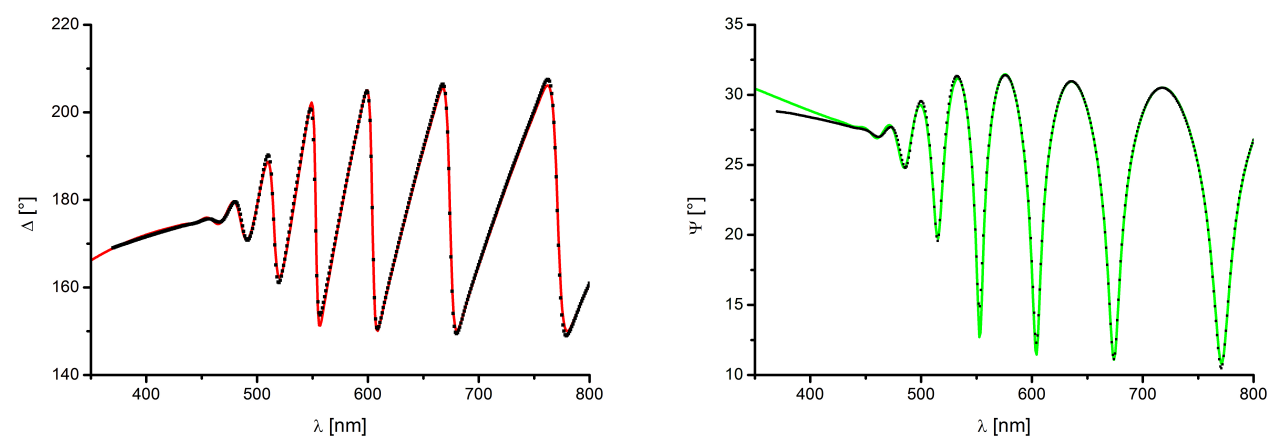

Abbildung 4.46 Delta/Psi-Wellenlängenspektrum von homogenen $\mathrm{As}_{2} \mathrm{~S}_{3}$ Film $(\mathrm{d}=960.5 \mathrm{~nm} \pm 0.1 \mathrm{~nm})$ auf Glas.

An diesem Beispiel wurden spektroskopische Messungen an $\mathrm{As}_{2} \mathrm{~S}_{3}$-Filmen bei einem $\mathrm{AOI}=55^{\circ} \mathrm{im}$ Bereich von 380-800 nm (1 nm Intervalle) durchgeführt. Für das Substrat (Glas) wurden die experimentellen Daten analog erhoben und die optischen Parameter mittels Cauchy-Dispersion beschrieben. Für das semitransparente Material $\mathrm{As}_{2} \mathrm{~S}_{3}$ wurde die Dispersion mittels Tauc-Lorentz-term (siehe Kapitel 2.6.4.6) beschrieben. In Abbildung 4.46 sieht man die gemessen und mittels Tauc-LorentzTerm simulierten Daten aus dem Ep4-Model im Vergleich.

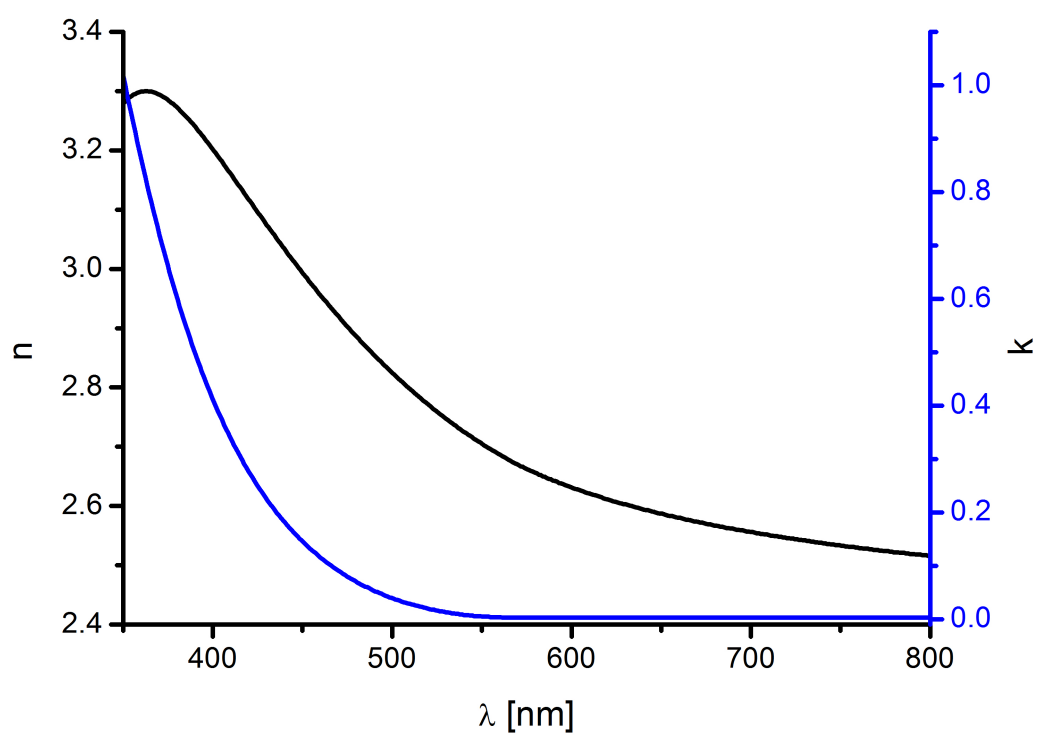

Abbildung 4.47 Dispersion von $\mathrm{As}_{2} \mathrm{~S}_{3}$. 


\begin{tabular}{|c|c|c|c|c|c|}
\hline Parameter & $\varepsilon_{\infty}$ & $\begin{array}{c}\mathrm{A} \\
{[\mathrm{eV}]}\end{array}$ & $\mathrm{E}_{0}[\mathrm{eV}]$ & $\begin{array}{c}\Gamma \\
{[\mathrm{eV}]}\end{array}$ & $\mathrm{E}_{\mathrm{g}}[\mathrm{eV}]$ \\
\hline \hline Wert & 2.741 & 81.433 & 3.858 & 1.716 & 2.180 \\
\hline
\end{tabular}

Tabelle 4.9 Tauc-Lorentz Parameter von $\mathrm{As}_{2} \mathrm{~S}_{3}$.

Die resultierenden Parameter des Tauc-Lorentz-Terms sind in Tabelle 4.9 zu finden. Die auf dem Tauc-Lorentz-Model basierenden $\mathrm{n}$ und $\mathrm{k}$ Werte sind in Abbildung 4.47 gezeigt. Es ist sehr schön zu erkennen, wie ab dem Bereich von $550 \mathrm{~nm}$ die Absorption des Materials immer stärker zum Tragen kommt. Die Oszillationen der Delta/PsiWerte, die aufgrund der Schichtdicke im transparenten Bereich zu sehen sind, nehmen deutlich ab. Ab einem Bereich von $450 \mathrm{~nm}$ sind keinerlei Informationen über die Schichtdicke des $\mathrm{As}_{2} \mathrm{~S}_{3}$ zu gewinnen. Aufgrund der eintretenden Absorption des Materials wird lediglich das einfallende Licht an der ersten Grenzfläche (Luft/ $\mathrm{As}_{2} \mathrm{~S}_{3}$ ) reflektiert, der zweite Reflex an der Grenzfläche zwischen Film und Substrat wird nicht detektiert. Eine exakte Bestimmung der Bandlücke des halbleitenden Materials konnte bei der Verwendung der Filterrads nicht erzielt werden. Die erhobenen optischen Parameter stimmen nahezu mit denen aus der Literatur überein, wobei marginale Abweichung aufgrund des Produktionsprozess möglich sind [53].

Den Einfluss der Bandbreite im Bezug auf Schichtdicken oberhalb von $1 \mu \mathrm{m}$ zeigt das folgende Beispiel. Dabei handelt es sich um einen homogenen Film von Siliziumnitrid auf Silizium-Substrat, der mittels Plasmagasphasenabscheidung hergestellt wurde.
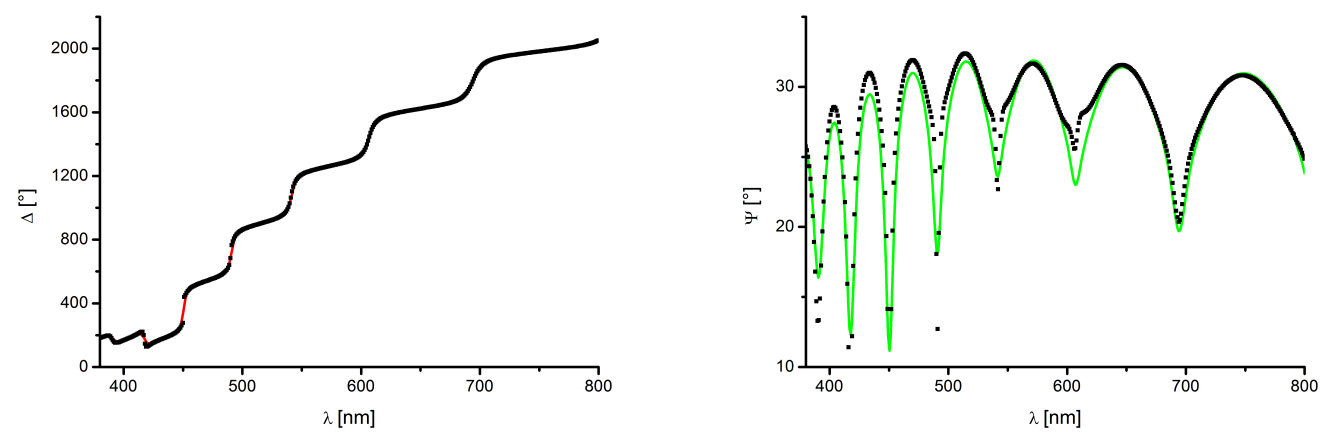

Abbildung 4.48 Delta/Psi-Wellenlängenspektrum $\mathrm{SiN}_{3}$ auf $\mathrm{Si}$ (100).

Das semitransparente Siliziumnitrid wurde mittels Tauc-Lorentz-Term beschrieben, die Ergebnisse der Modelleirung sind in Tabelle 4.10 gezeigt. Im Wellenlängenspektrum der ellipsometrischen Parameter Delta und Psi (Abbildung 4.48) ist im Vergleich zum $\mathrm{As}_{2} \mathrm{~S}_{3}$ kein deutlicher Übergang zwischen dem transparenten bis hin zum semitransparenten Verhalten zu erkennen. Das liegt an dem deutlich kleineren Absorptionskoeffizienten (Abbildung 4.49), so dass die aufgrund der Schichtdicke des $\mathrm{SiN}_{3}$ auftretenden Oszillationen im Spektrum erhalten bleiben. Erst unter Einbeziehung der Bandbreite der verwendeten Lichtquelle war es möglich, die optischen 
Parameter des Materials zu generieren, da gerade im UV-Bereich der Effekt der Bandbreite zum Tragen kommt.

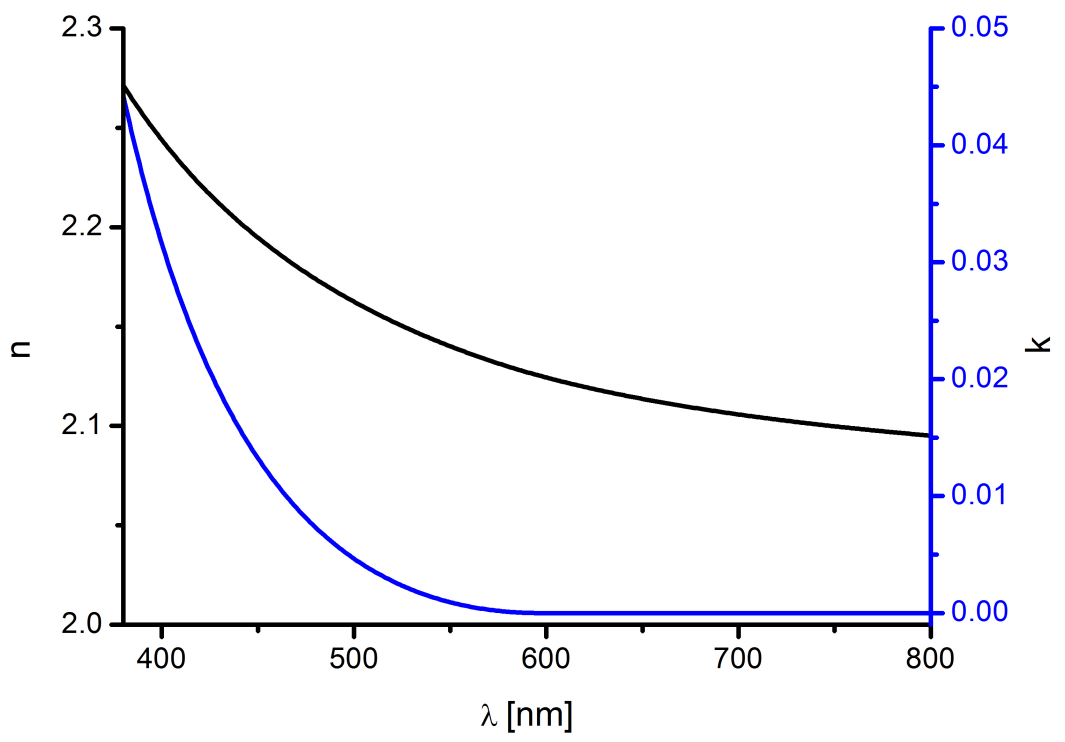

Abbildung 4.49 Dispersion von $\mathrm{SiN}_{3}$ auf Si (100).

\begin{tabular}{|c|c|c|c|c|c|}
\hline Parameter $\varepsilon_{\infty}$ & $\begin{array}{c}\mathrm{A} \\
{[\mathrm{eV}]}\end{array}$ & $\mathrm{E}_{0}[\mathrm{eV}]$ & $\begin{array}{c}\Gamma \\
{[\mathrm{eV}]}\end{array}$ & $\mathrm{E}_{\mathrm{g}}[\mathrm{eV}]$ \\
\hline \hline Wert & 3.013 & 20.991 & 5.291 & 1.339 & 2.075 \\
\hline
\end{tabular}

Tabelle 4.10 Tauc-Lorentz Parameter von $\mathrm{SiN}_{3}$. 


\title{
Neue Plastic Solar Cell Materialien DPIDT-2T
}

In der Forschung werden immer neue leitfähige Polymere synthetisiert, die noch effizienter das Sonnenlicht absorbieren. Hier wird ein Dihydropyrroloindoledion-derivat, dass durch Copolymerisation mit Thiophen ein breites Absoprtionsverhalten im visuellen Bereich zeigt [48]. Neben den optischen Parametern der dünnen Polymerfilme (Herstellung siehe Probenpräparation) sollte der Einfluß des Annealings ${ }^{32}$ betrachtet werden. Dazu wurden die einzelnen Proben an 9 Positionen spektroskopisch im Bereich von 380-800 nm (2 nm Intervalle) bei einem Einfallswinkel von $50^{\circ}$ vermessen. In Abbildung 4.50 sind die Spektren der Proben bei einer Rotationsgeschwindigkeit von $500 \mathrm{rpm}$ im Vergleich gezeigt. Beide Proben besitzen laut Datenanalyse in etwa die gleiche Schichtdicke $\left(d_{\text {as cast }}=98.8 \pm 0.2 \mathrm{~nm}, d_{\text {annealed }}=99.4 \pm 0.5 \mathrm{~nm}\right)$.

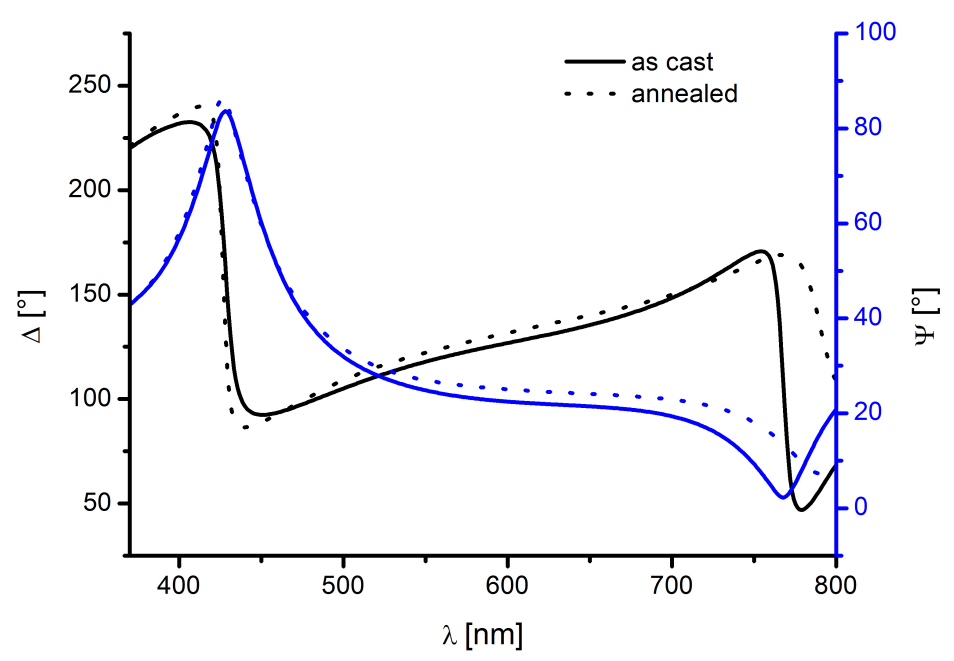

\begin{abstract}
Abbildung 4.50 Vergleich der Delta/Psi-Wellenlängenspektren von DPIDT-T2 (500 rpm).
\end{abstract}

Bei der Datenanalyse der Proben wurde das Substrat Si (100) mit einer $\mathrm{SiO}_{2}$-Schicht $(\mathrm{d}=3.3 \mathrm{~nm})$ unter Verwendung der Dispersionen aus der Datenbank simuliert. Das optische Verhalten des Dihydropyrroloindoledion-derivats wurde durch eine uniaxiale anisotrope Schicht modelliert. Die Ergebnisse für die Schichtdicke in Abhängigkeit der Rotationsgeschwindigkeit sind in Tabelle 4.11 zusammengefasst. 


\begin{tabular}{|c|c|c|}
\hline$[\mathrm{rpm}]$ & $\mathrm{d}$ (as cast) $[\mathrm{nm}]$ & $\mathrm{d}$ (annealed) $[\mathrm{nm}]$ \\
\hline \hline 500 & $98.8 \pm 0.2$ & $99.6 \pm 0.5$ \\
\hline 1000 & $64.4 \pm 0.1$ & $52.1 \pm 0.3$ \\
\hline 1500 & $48.8 \pm 0.2$ & $43.3 \pm 0.3$ \\
\hline 2000 & $47.1 \pm 0.2$ & $43.6 \pm 0.3$ \\
\hline
\end{tabular}

Tabelle 4.11 Schichtdicke von DPIDT-2T Filmen bei variierender Rotationsgeschwindigkeit beim Herstellungsprozess.

Die Dispersion des Materials wurde durch die Kombination von einem Tauc-LorentzTerm mit einem Gauss-Term beschrieben. Die resultierenden Werte für den Brechungsindex und dem Absoprtionskoeffizienten sind in Abbildung 4.51 gezeigt. Die optischen Parameter in der Probenebene werden als durchgezogene und senkrecht zur Probe als gestrichelte Linie dargestellt.
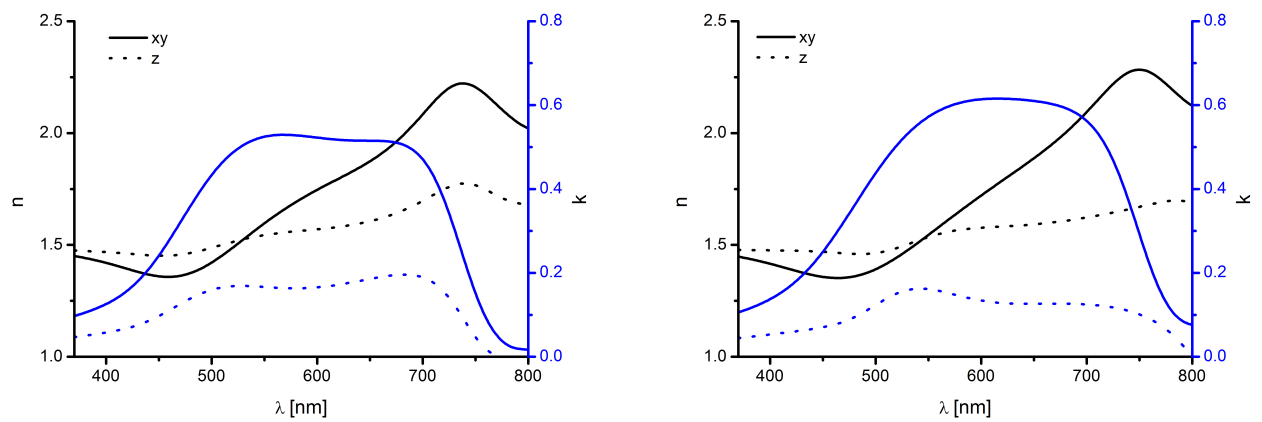

Abbildung 4.51 Dispersion DPIDT-T2 (as cast, annealed)

Auf der linken Seite der Abbildung ist die Dispersion von DPIDT-2T aller Proben (as cast) abgebildet. Wie in der Literatur bereits beschrieben, zeigt das Material eine sehr breite Absorption im visuellen Bereich. Vergleicht man die erhobenen optischen Parameter mit denen der ausgeheizten Proben (Abbildung, rechts), verbreitert sich die Absorption deutlich und der Wert für die Absoprtion in x- und y-Richtung nimmt zu. Betrachtet man den Brechungsindex ändern sich besonders ab $650 \mathrm{~nm}$ die Werte. Es ist zu vermute, dass dies an einer besseren Ausrichtung der Moleküle aufgrund des annealings innerhalb des Films liegt. Die zugrunde liegenden Modelparameter sind in Tabelle 4.12 gezeigt. 


\begin{tabular}{|c|c|c|c|c|}
\hline behandelt & Model & Parameter & xy & $\mathrm{Z}$ \\
\hline \multirow{8}{*}{ as cast } & & $\mathrm{eps}_{\infty}$ & $2.463 \pm 0.04$ & $2.286 \pm 0.009$ \\
\hline & \multirow{4}{*}{ Tauc-Lorentz } & $\mathrm{A}[\mathrm{eV}]$ & $49.579 \pm 0.930$ & $30.092 \pm 3.303$ \\
\hline & & $\mathrm{E}_{0}[\mathrm{eV}]$ & $1.681 \pm 0.002$ & $1.655 \pm 0.007$ \\
\hline & & $\Gamma[\mathrm{eV}]$ & $0.253 \pm 0.004$ & $0.223 \pm 0.019$ \\
\hline & & $\mathrm{E}_{\mathrm{g}}[\mathrm{eV}]$ & $1.566 \pm 0.002$ & $1.593 \pm 0.007$ \\
\hline & \multirow{3}{*}{ Gauß } & $\mathrm{A}$ & $0.86 \pm 0.01$ & $0.19 \pm 0.01$ \\
\hline & & $\mathrm{E}_{\mathrm{c}}[\mathrm{eV}]$ & $2.23 \pm 0.004$ & $2.41 \pm 0.01$ \\
\hline & & $\mathrm{B}[\mathrm{eV}]$ & $0.71 \pm 0.003$ & $0.49 \pm 0.03$ \\
\hline \multirow{8}{*}{ annealed } & & $\mathrm{eps}_{\infty}$ & $2.498 \pm 0.011$ & $2.267 \pm 0.017$ \\
\hline & \multirow{4}{*}{ Tauc-Lorentz } & $\mathrm{A}[\mathrm{eV}]$ & $56.755 \pm 2.089$ & $518.780 \pm 26.103$ \\
\hline & & $\mathrm{E}_{0}[\mathrm{eV}]$ & $1.646 \pm 0.003$ & $1.499 \pm 0.017$ \\
\hline & & $\Gamma[\mathrm{eV}]$ & $0.247 \pm 0.007$ & $0.014 \pm 0.001$ \\
\hline & & $\mathrm{E}_{\mathrm{g}}[\mathrm{eV}]$ & $1.552 \pm 0.004$ & $1.543 \pm 0.010$ \\
\hline & \multirow{3}{*}{ Gauß } & $\mathrm{A}$ & $1.02 \pm 0.02$ & $0.20 \pm 0.01$ \\
\hline & & $\overline{\mathrm{E}_{\mathrm{c}}[\mathrm{eV}]}$ & $2.09 \pm 0.01$ & $2.30 \pm 0.01$ \\
\hline & & $\mathrm{B}[\mathrm{eV}]$ & $0.84 \pm 0.01$ & $0.38 \pm 0.03$ \\
\hline
\end{tabular}

Tabelle 4.12 Modelparameter für DPIDT-2T Filme.

\section{N2200}

Ein anderer Vertreter dieser n-Typ Halbleiter Klasse, ein Copolymer aus Thiophen und einem Naphthalendicarboximidderivat, wurde ebenfalls untersucht. In diesem Falle lag der Fokus auf der Frage, welchen Einfluß das Lösungsmittels habe.

Bei dieser Probe wurde das Copolymer in Xylene gelöst. Im ellipsometrischen Kontrastbild (Abbildung 4.54 oben) ist eine deutliche Inhomogenität der Oberfläche zu erkennen. Es wurden 34 ROIs (10x Objektiv) gleichzeitig gemessen. Die Messungen wurden bei einem festen Einfallswinkel von $50^{\circ}$ und im Wellenlängenbereich von 380-800 nm (4 nm Intervalle) durchgeführt. Wie anhand des Kontrastbildes zu erwarten, zeigen die Wellenlängenspektren der ROI-basierten Messungen marginale Abweichungen. In Abbildung 4.52 sind die gemessenen und modellierten Wellenlängenspektren von ROI 5 und 20 gegenübergestellt.
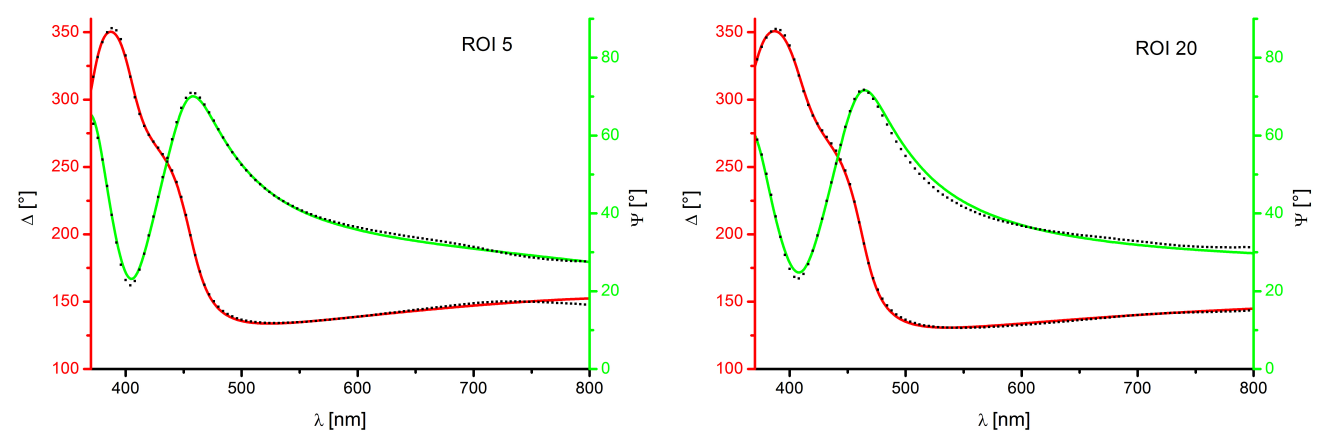

Abbildung 4.52 Gemessene und simultierte Delta/PsiWellenlängenspektren von N2200 (Xylene) auf Si (100). 
Die Dispersion des Copolymers (isotrop) wurde durch einen Tauc-Lorentz- und einen Lorentz-Term modelliert, deren Parameter neben der Schichtdicke gefittet wurden. Dabei beschreibt Tauc-Lorentz die Halbleitereigenschaften und Lorentz die Dotierung des Materials. Die resultierenden Dispersionen von ROI 5 (d $=76.7 \mathrm{~nm}$, Linie) und ROI 20 (d = $82.5 \mathrm{~nm}$, Punkte) sind in Abbildung 4.53 gezeigt.

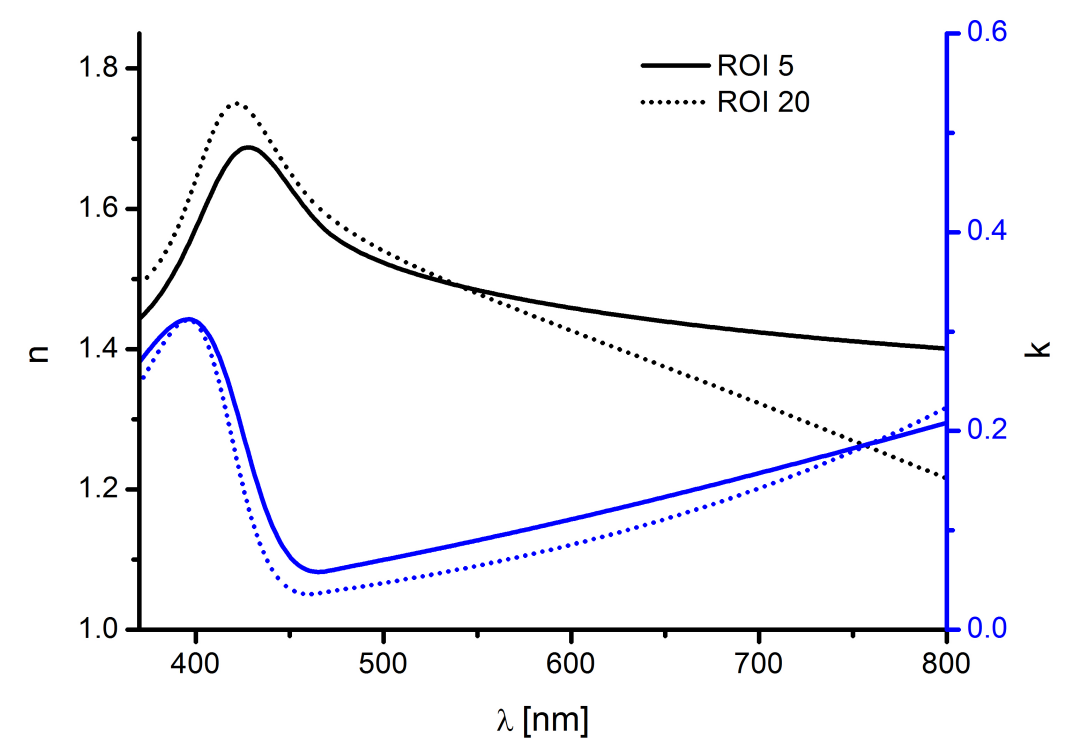

Abbildung 4.53 Dispersion von N2200 (Xylene) auf Si (100) der ROI-basierten Messungen.

Im direkten Vergleich der optischen Parameter der beiden ROIs sieht man einen deutlichen Unterschied. Dabei zeigt das Material von ROI 5 eine stärker ausgeprägte Absorption im visuellen Bereich, die im Vergleich zu ROI 20 in Richtung des IR-Bereich nicht so stark ansteigt. Der Kontrast im Bild kann einerseits durch die leicht verschiedene Schichtdicke als auch durch die unterschiedlichen optischen Eigenschaften des Materials kommen. Der Verdacht liegt nahe, dass der Film aufgrund des Lösungsmittels kristalline Teilbereiche aufweist [54].

Basierend auf dieser Modellierung wurden alle ROIs als Mischung aus beiden Zuständen beschrieben und durch eine effektive Beschreibung nach Bruggemann (Kapitel 2.6.5) ersetzt. Neben der Schichtdicke wurde zusätzlich der fraction guest factor als freier Parameter mit berechnet. Eine graphische Übersicht der sich ergebenen Werte und das dazugehörige Kontrastbild $(\lambda=440 \mathrm{~nm})$ sind in Abbildung 4.54 gezeigt. 


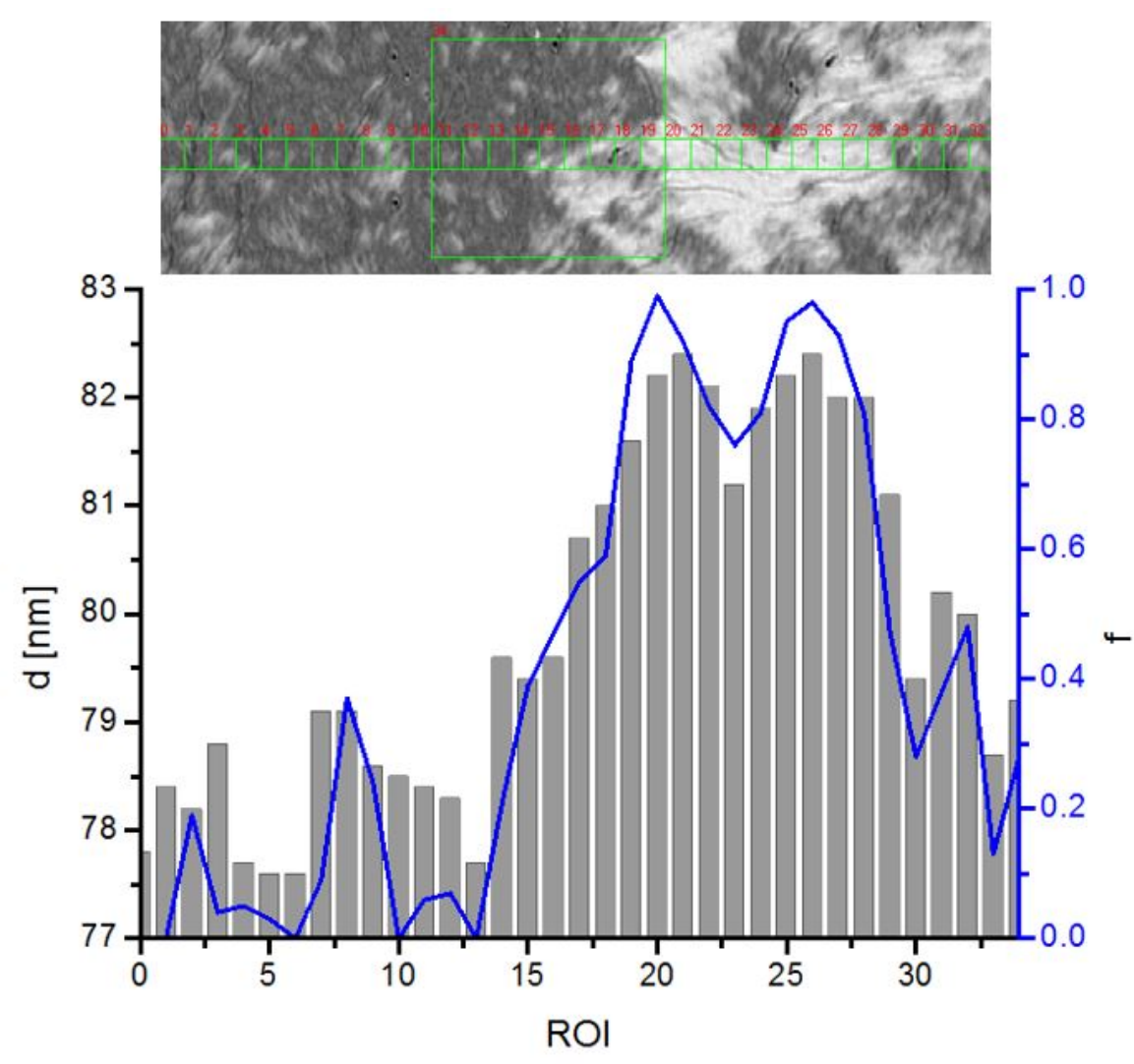

Abbildung 4.54 Ellipsometrisches Kontrastbild (10x Objektiv, $\lambda=440 \mathrm{~nm}$ ) und ROI Profil N2200 (Xylene) auf Si (100).

Auf den ersten Blick ist zwar ein leichter Zusammenhang zwischen Schichtdicke und fraction guest factor zu erkennen, schaut man aber genauer hin, ist dies nicht der Fall. Generell erhält man aus dem Ep4-Model eine Korellation beider Parameter von maximal $84 \%$.

Analog dazu wurde ein Film des Copolymers auf Si (100) aus eine Polymerösung mit Chlorbenzol hergestellt. Die ellipsometrischen Kontrast-Bilder zeigten eine homogene Oberfläche. In Abbildung 4.55 sind die experimentellen und modellierten Messdaten visualisiert. 

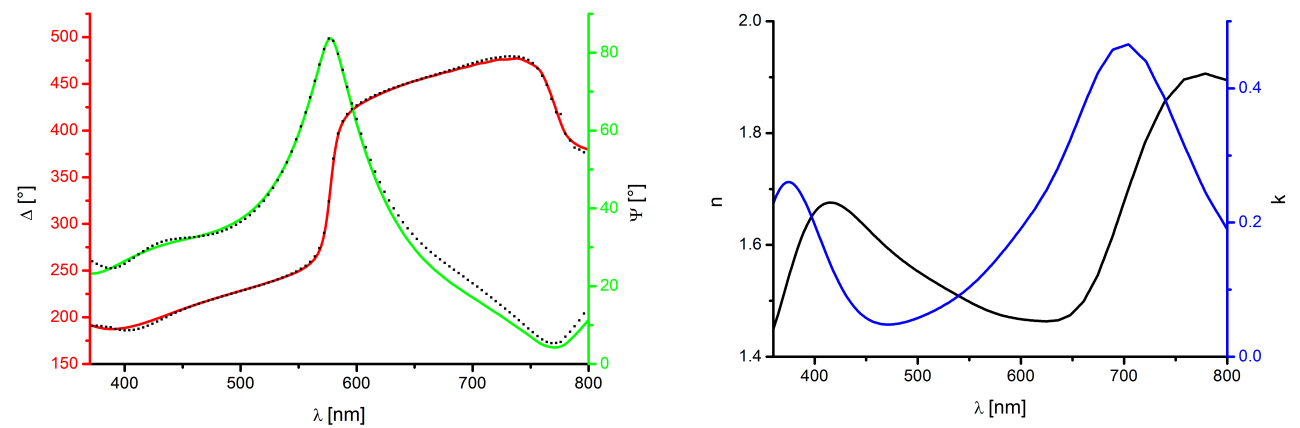

\begin{abstract}
Abbildung 4.55 Delta/Psi-Werte in Abhängikeit von der Wellelänge und der dazugehörigen Dispersion des N2200-Films (d $=125.6 \pm 10.7 \mathrm{~nm}$, Chlorbenzol) auf Si (100).
\end{abstract}

Im Vergleich zur vorherigen Dispersion des selben Materials zeigt sich eine deutliche Ausprägung eines Absorptionspeaks bei $700 \mathrm{~nm}$. Der UV-Bereich der Dispersion ähnelt sehr stark dem Verlauf aus Abbildung 4.53 des teilkristallinen N2200-Films. Vergleicht man die beiden Dispersionen mit denen in der Literatur, zeigt das Copolymer aus Chlorbenzol eine gute Übereinstimmung der Absorption, der dort mittels UV/VIS-Spektroskopie bestimmten [44]. Die Lage der Absorptionspeaks sind deckungsgleich mit denen aus der UV/VIS-Spektroskopie. Lediglich die Intensität der Absorption weicht beim N2200 Film aus Chlorbenzol $(\mathrm{d}=125.6 \pm 10.7 \mathrm{~nm}) \mathrm{im}$ Vergleich zum N2200 Film $(\mathrm{d}=90 \mathrm{~nm})$ aus der Literatur ab.

\title{
PTB7
}

Thiophene-derivate haben sich bei Plastik Solar Zellen als p-Typ Halbleiter bewährt. Besonders die Effizienz bei der Energieumwandlung ist eines der Hauptcharakteristika, die es zu es zu verbessern gilt. Ein neues Polymer bestehend aus einer Thieno[3,4b]thiophen und Benzodithiophen-Einheit zeigt gemäß Literatur eine starke Absorption im Bereich von 550-750 nm bei UV/VIS-Messungen [38]. Die optischen Parameter dieses neuen Donormaterials wurde spektroskopisch mittels abbildender Ellipsometrie untersucht. Dazu wurde die Probe an 9 Positionen im Bereich von 380-800 nm (4 nm Abstand) bei einem Einfallswinkel von 50 gemessen. Bei der Auswertung der Spektren wurde für die optische Beschreibung des Thiophenderivates eine Kombination aus Lorentz und Drude gewählt. Die gemessenen Delat/Psi-Werte und die darauf basierende Dispersion für PTB-7 sind in Abbilung 4.56 gezeigt.

Die Schichtdicke des PTB7 Films beträgt $44.7 \pm 1.2 \mathrm{~nm}$ und ist verhältnismässig dünn. Die in der Literatur beschriebene breite Absorption des leitfähigen Filmmaterials kann hier nicht gefunden werden [38]. Bei der Modelleirung ergibt sich eine deutliche Absorption bei $750 \mathrm{~nm}$ in der Dispersion, die durch den Lorentz-Term beschrieben wird.

\section{PCE10}

Nachdem bei Filmen mit PTB7 eine Energieeffizienz von $7.6 \%$ errreicht wurde, zeigt die Seitenkettenmodifikation der Benzodithiophen-Einheit des Polymers mit 2 Thiophenen eine deutliche Steigerung $(9.0 \%$ ) [38, 64]. Als bessere Alternative des 

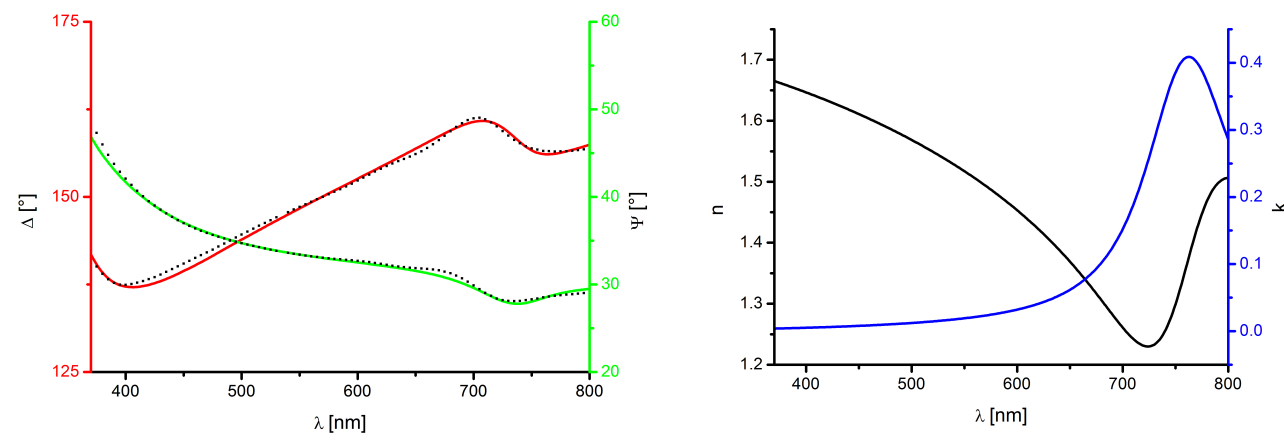

Abbildung 4.56 Delta/Psi-Werte in Abhängikeit von der Wellelänge und der dazugehörigen Dispersion PTB7-Films (d $=44.7 \pm 1.2 \mathrm{~nm})$ auf Si (100) .

leitfähigen Polymers wurde ein PCE10 Film auf Si (100) mittels spektroskopischer Ellipsometrie untersucht. Die Schichtdicke und Parameter des Modells (3 Lorentz + 1 Drude Term) für PCE10 wurden anhand der an 9 Positionen auf der Probe aufgenommen Daten berechnet. Die Ergebnisse der Datenanalyse und der experimentellen Daten für Delta/Psi sind in Abbildung $4.57 \mathrm{zu}$ sehen.
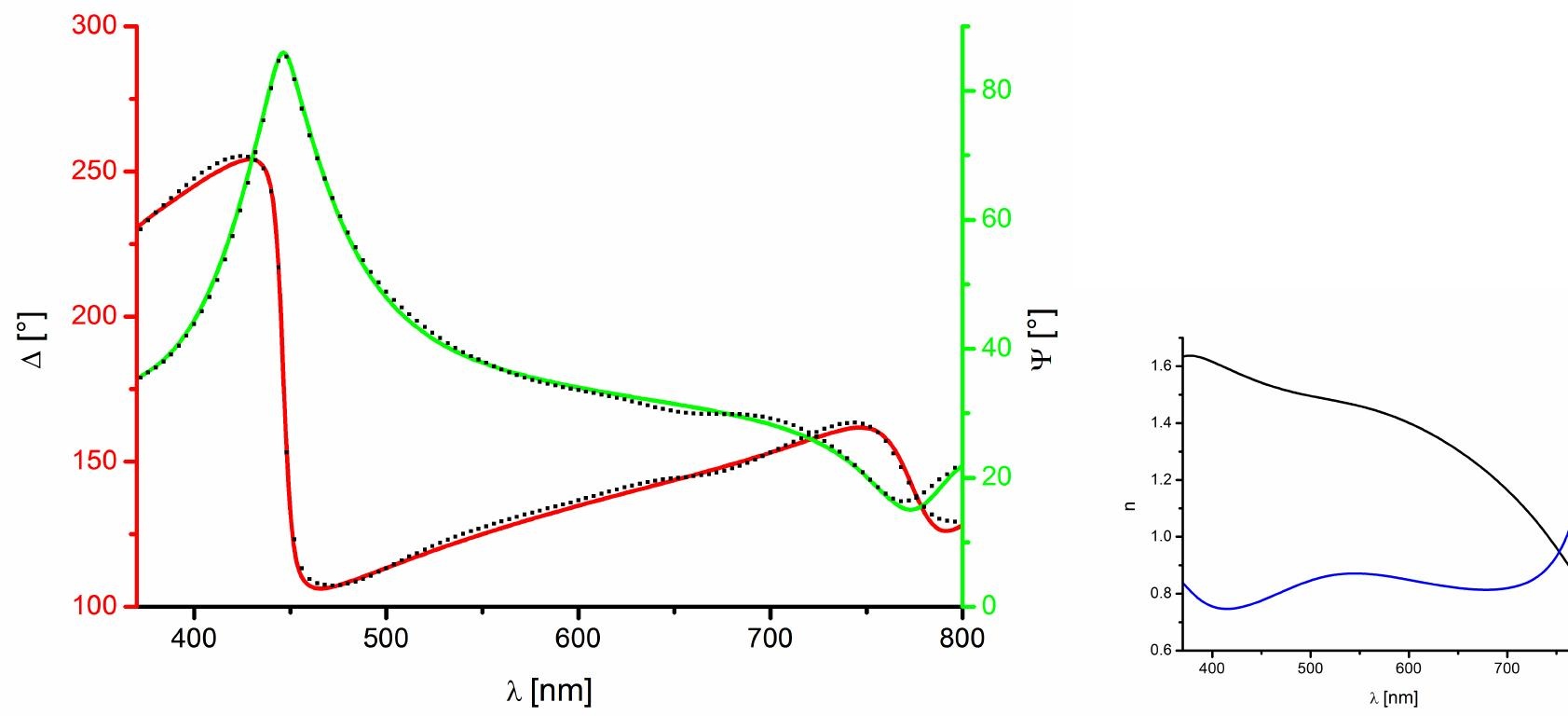

Abbildung 4.57 Delta/Psi-Werte in Abhängikeit von der Wellenlänge und der dazugehörigen Dispersion des PCE10-Films $(\mathrm{d}=84.2 \pm 1.2 \mathrm{~nm})$ auf Si $(100)$.

Als mittlere Schichtdicke für PCE10 wurde $84.2 \pm 1.2 \mathrm{~nm}$ erhalten. Die Oberfläche der Probe war im Kontrastbild homogen. Die gemittelten Werte sind in Tabelle 4.13 gezeigt. 


\begin{tabular}{|c|c|c|}
\hline Model & Parameter & Wert \\
\hline \hline & $\mathrm{eps}_{\infty}$ & $3.273 \pm 0.023$ \\
\hline Lorentz 1 & $\mathrm{E}_{0}[\mathrm{eV}]$ & $1.525 \pm 0.002$ \\
\hline & $\mathrm{A}\left[\mathrm{eV}^{2}\right]$ & $0.151 \pm 0.007$ \\
\hline & $\Gamma[\mathrm{eV}]$ & $0.112 \pm 0.003$ \\
\hline Lorentz 2 & $\mathrm{E}_{0}[\mathrm{eV}]$ & $2.384 \pm 0.004$ \\
\hline & $\mathrm{A}\left[\mathrm{eV}^{2}\right]$ & $1.598 \pm 0.028$ \\
\hline & $\Gamma[\mathrm{eV}]$ & $1.352 \pm 0.020$ \\
\hline Lorentz 3 & $\mathrm{E}_{0}[\mathrm{eV}]$ & $3.494 \pm 0.014$ \\
\hline & $\mathrm{A}\left[\mathrm{eV}{ }^{2}\right]$ & $1.108 \pm 0.064$ \\
\hline & $\Gamma[\mathrm{eV}]$ & $0.681 \pm 0.029$ \\
\hline Drude & $\mathrm{A}[\mathrm{eV}]$ & $6.96 \pm 0.050$ \\
\hline & $\Gamma[\mathrm{eV}]$ & 0 \\
\hline
\end{tabular}

Tabelle 4.13 Modelparameter für PCE10.

Ähnlich wie beim PTB7 sollte laut Literatur das Material PCE10 eine breite Absorption von 600-700 nm zeigen. In der berechneten Dispersion erhält man lediglich eine angedeutete Absorption außerhalb des Messbereichs $(\lambda>800 \mathrm{~nm})$ [64]. Somit führt die Seitenkettenmodifikation der Benzodithiophen-Einheit zu einer Verschiebung der Absorptionsbande in den NIR-Bereich. Zusätzlich zeig sich noch ein breite leicht ausgeprägte Absorptionsbande im visuellen Bereich $(\lambda=520 \mathrm{~nm})$. .

\section{CPF-4}

Um das gesamte Strahlungsprofil der Sonne zu nutzen, werden ebenfalls Supramoleküle in Form von modifizierten Fullerenen anstelle von Thiophenen bei Plastik Solar Zellen benutzt. Das CPF-4 besteht aus einem Carotenoid, das atomar über ein Porphyrinmolekül mit dem Fullerene verbunden ist [63]. Die experimentellen und simulierten Daten der spektroskopischen Messungen sind in Abbildung 4.58 gezeigt.
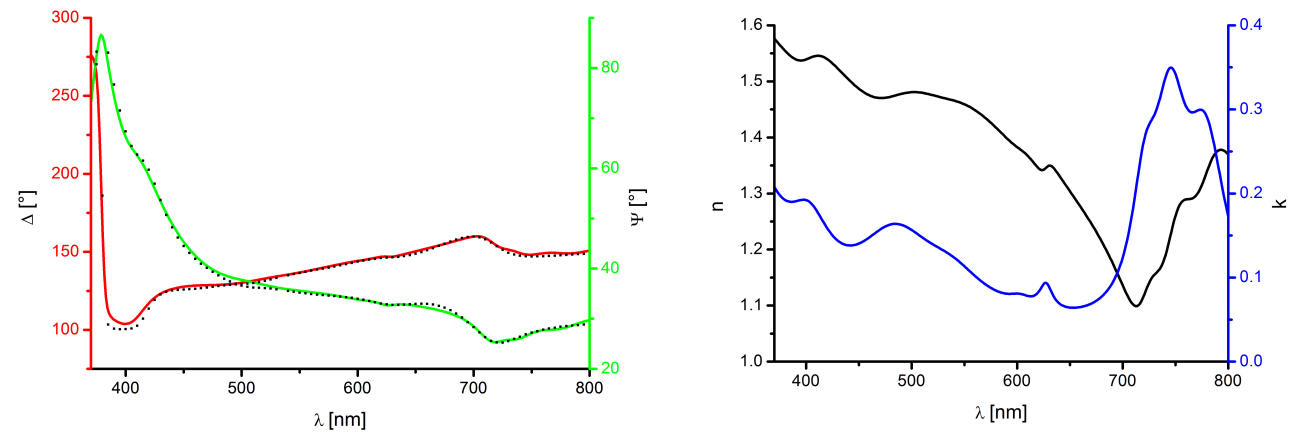

Abbildung 4.58 Delta/Psi-Werte in Abhängikeit von der Wellelänge und der dazugehörigen Dispersion CPF-Films $(\mathrm{d}=67.6 \pm 1.1 \mathrm{~nm})$ auf Si (100). 
Wie in der Literatur bereits beschrieben zeigt das Supramolekül die Absorptionsbanden der einzelnen Teilmoleküle (Fulleren, Carotenoid und Porphyrin) [63]. Zusätzlich zu den charakteristischen Banden zeigt das Supramolekül eine starke Absorption bei $750 \mathrm{~nm}$, die in etwa der der Thiophenderivaten entspricht.

\title{
Polyblend
}

Neben den reinen Schichtmaterialien wurden auch Gemische sogenannte Polyblends bestehend aus n- und p-Typ Halbleitermaterialien spektroskopisch untersucht und neben der Schichtdicke deren Dispersion erhalten.

\section{PTB7:N2200}

In der Abbildung 4.59 wird das Wellenlängensprektum sowie die Dispersion der Polyblend von PTB7:N2200 im Verhältnis von 1:1.2 gezeigt. Die charakteristischen Verläufe von $\mathrm{n}$ un $\mathrm{k}$ der reinen Materialien bleiben erhalten.
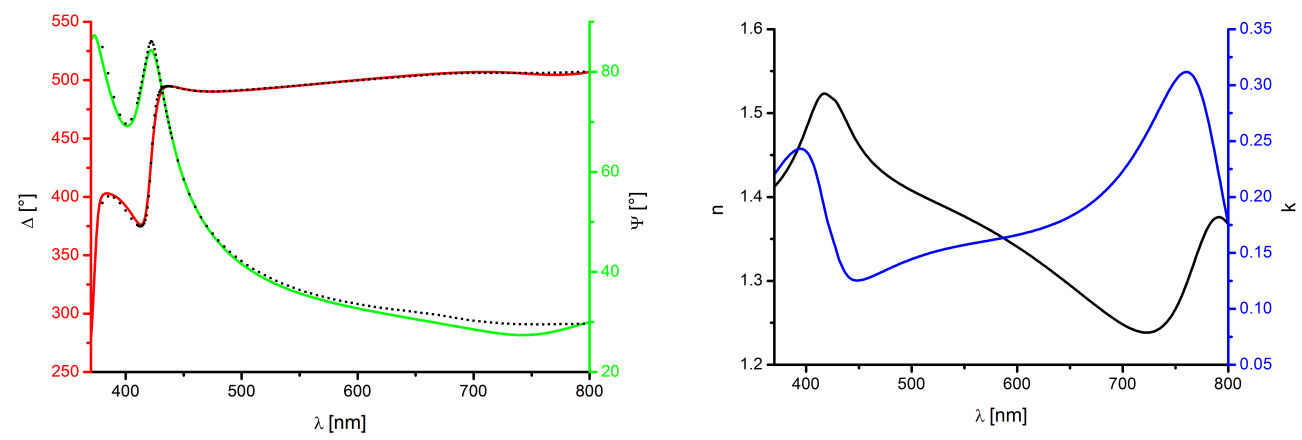

\begin{abstract}
Abbildung 4.59 Delta/Psi-Werte in Abhängikeit von der Wellelänge und der dazugehörigen Dispersion PTB7:N2200-Films (1:1.2, $\mathrm{d}=85.6 \pm 2.9 \mathrm{~nm})$ auf Si (100).
\end{abstract}

\section{PCE-10:N2200}

Das experimentellen und simulierten Daten der spektroskopischen Messungen der Polyblend von PCE-10:N2200 im Verhältnis von 1:1 wird in Abbildung 4.60 gezeigt. Im Vergleich zur Dispersion der reinen Schichtmaterialien ist die Absorption deutlich stärker ausgeprägt.

\section{CPF-4:N2200}

Die Dispersion und das Delta/Psi-Spektrum der Polyblend von CPF-4:N2200 im Verhältnis von 2:1 ist in Abbildung $4.61 \mathrm{zu}$ sehen. Dabei ist die charakteristische Bande in der Extinktion des CPF-4 in Richtung höherer Wellenlängen verschoben und deutlich stärker ausgeprägt. 

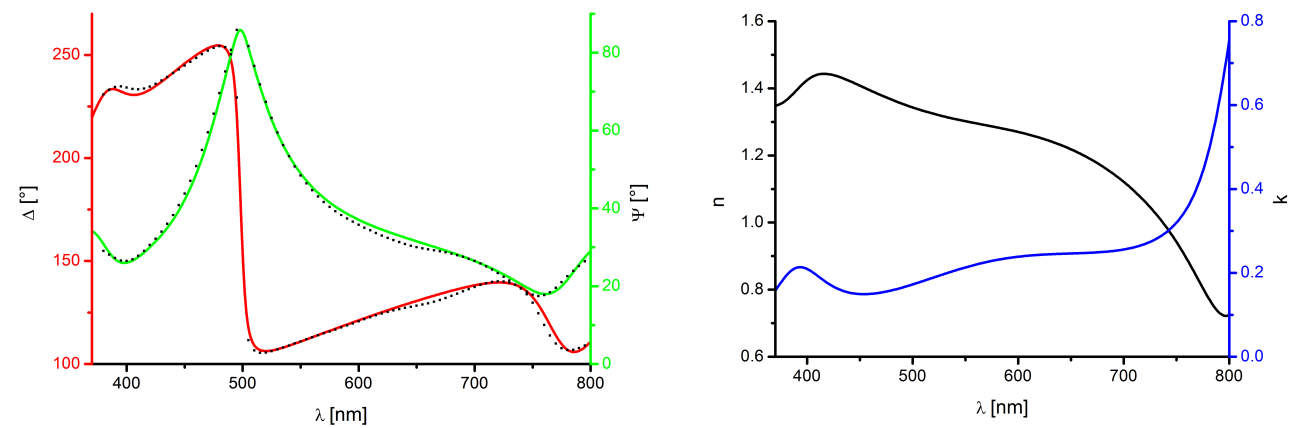

Abbildung 4.60 Delta/Psi-Werte in Abhängikeit von der Wellelänge und der dazugehörigen Dispersion PCE10:N2200-Films (1:1, $\mathrm{d}=120.4 \pm 3.8 \mathrm{~nm})$ auf Si (100).
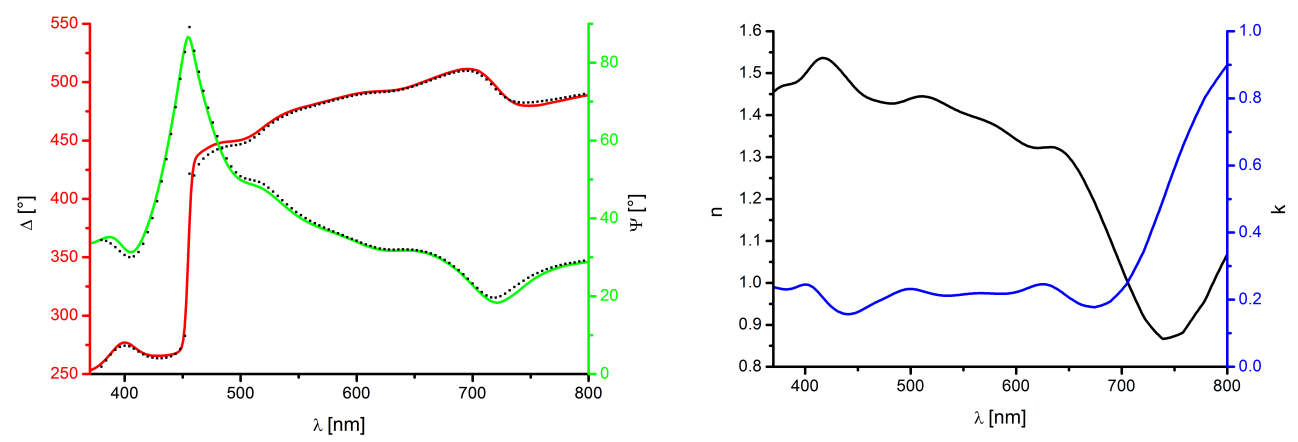

Abbildung 4.61 Delta/Psi-Werte in Abhängikeit von der Wellelänge und der dazugehörigen Dispersion CPF-4:N2200-Films (2:1, $\mathrm{d}=98.8 \pm 1.5 \mathrm{~nm})$ auf Si (100).

\subsubsection{UV/NIR-Erweiterung}

Als Erstes wurde das Ep3-SE System in den NIR-Bereich erweitert. Das Filterrad wurde um Transmissionsfilter für den NIR-Bereich ergänzt und eine NIR-Kamera implementiert. Nach dem Wechsel vom Filterrad zum Monochromator als dispersives Element, wurde um den kompletten Wellenlängenbereichvon 250-1700 nm abzudecken, wurde ein triaxialer Monochromator eingebunden, der zwischen den jeweiligen Gitter je nach gewünschter Wellenlänge wechselt. Aufgrund der abbildenden Methode musste zusätzlich ein Objektiv (Nanochromat NC2 12.5x/0,25, Technologie Manufaktur) entwickelt werden, das über den gesamten Wellenlängenbereich transparent ist. Zusätzlich wurd die Standardkamera durch eine UV/VIS Kamera und einer NIR Kamera ersetzt, zwischen denen je nach genutzter Wellenlänge mittels Klappspiegel hin und her geschaltet wird.

Die Erweiterung des Wellenlängenbereichs wird am Beispiel von Graphenflocken gezeigt. Als Startpunkt dienen die ersten spektroskopischen Messungen mittels abbildender Ellipsometrie, die von Wurstbauer et al. 2010 in Appl. Phys. Lett. veröffentlicht wurden [62]. An einer Monolage von Graphen auf Si (100) mit $300 \mathrm{~nm}$ 
$\mathrm{SiO}_{2}$ wurden Messungen im Bereich von 380-960 nm (20 nm Intervalle) und bei Einfallswinkeln von $40-49^{\circ}$ ( $3^{\circ}$ Intervalle) durchgeführt.

Wie in der Literatur beschrieben, wurden anhand des Drude Models die optischen Parameter des Graphens bestimmt. Zwei Jahre darauf modellierte Matkovic et al. in seiner Veröffentlichung die Dispersion von Graphen mittels Fanoresonanz [42]. Auch diese Untersuchungen wurden mittels spektroskopisch abbildender Ellipsometrie im Bereich von 360-800 nm durchgeführt.

In Abbildung 4.62 sind die Delta/Psi-Spektren einer Graphenprobe auf Si (100) mit $292.3 \mathrm{~nm} \mathrm{SiO}_{2}$ gezeigt. Die Messungen wurden bei einem festen Winkel von $50^{\circ}$ über den gesammten spektralen Bereich $(\lambda=250-1700 \mathrm{~nm})$ in $5 \mathrm{~nm}$ Intervallen aufgenommen. Wie in der Delta-Map gezeigt wurden die Delta/Psi-Wertepaare für das Substrat, die Monolage und Mehrfachlage des Graphens simultan gemessen.
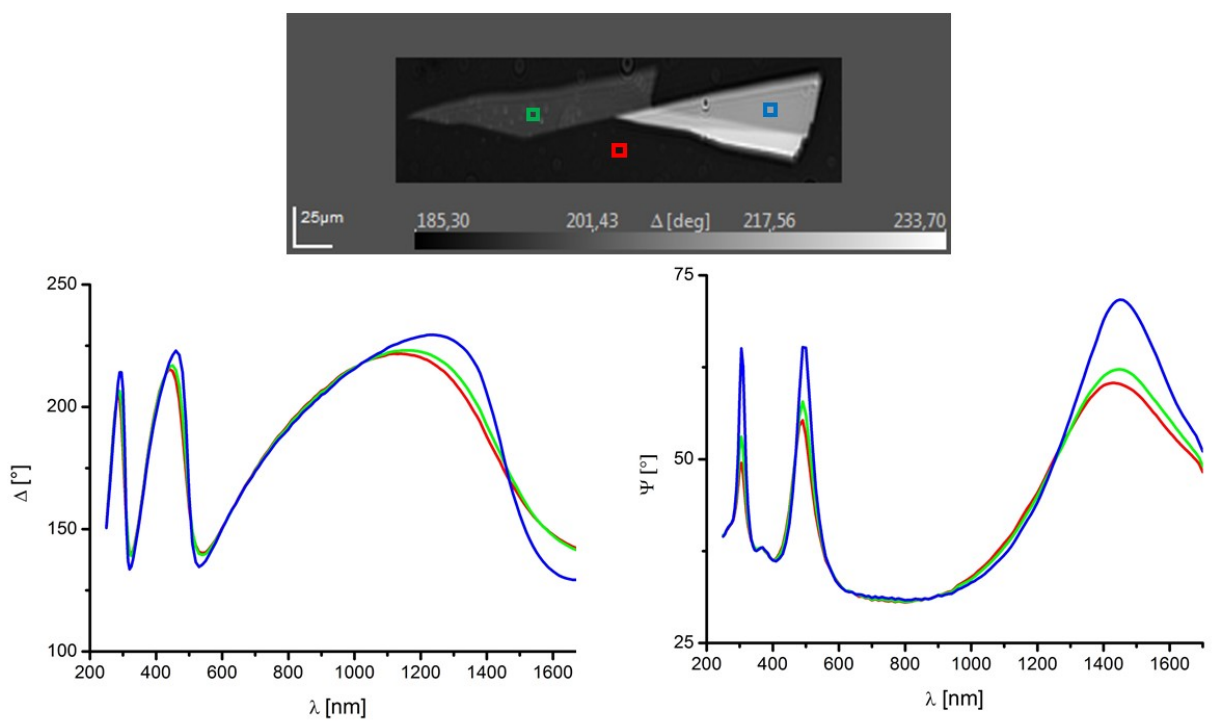

Abbildung 4.62 UV/NIR-Messung von Graphenflocken auf Si (100) mit $392.3 \mathrm{~nm} \mathrm{SiO}_{2}$ mit Delta-Map bei $480 \mathrm{~nm}$.

Neben der Schichtdicke der Mono- und Trilage der Graphenflocke wurde zusätzlich die Dispersion von Graphen im UV-NIR-Bereich bestimmt. Als Model zur Bestimmung der Schichdicke wurde anfangs das Fano-Model aus der Literatur genutzt. Dabei traten besonders im NIR-Bereich deutliche Abweichungen auf, weshalb für die Bestimmung der Schichtdicke von Graphen $\left(\mathrm{d}_{1 \mathrm{~L}}=0.34 \pm 0.2 \mathrm{~nm}\right.$, $\left.\mathrm{d}_{3 \mathrm{~L}}=1.02 \pm 0.2 \mathrm{~nm}\right)$ nur die Daten von 360-800 nm genutzt wurden. Alternativ dazu wurde eine Modelbeschreibung aus einem Drude- und Lorentz-Term gewählt. 


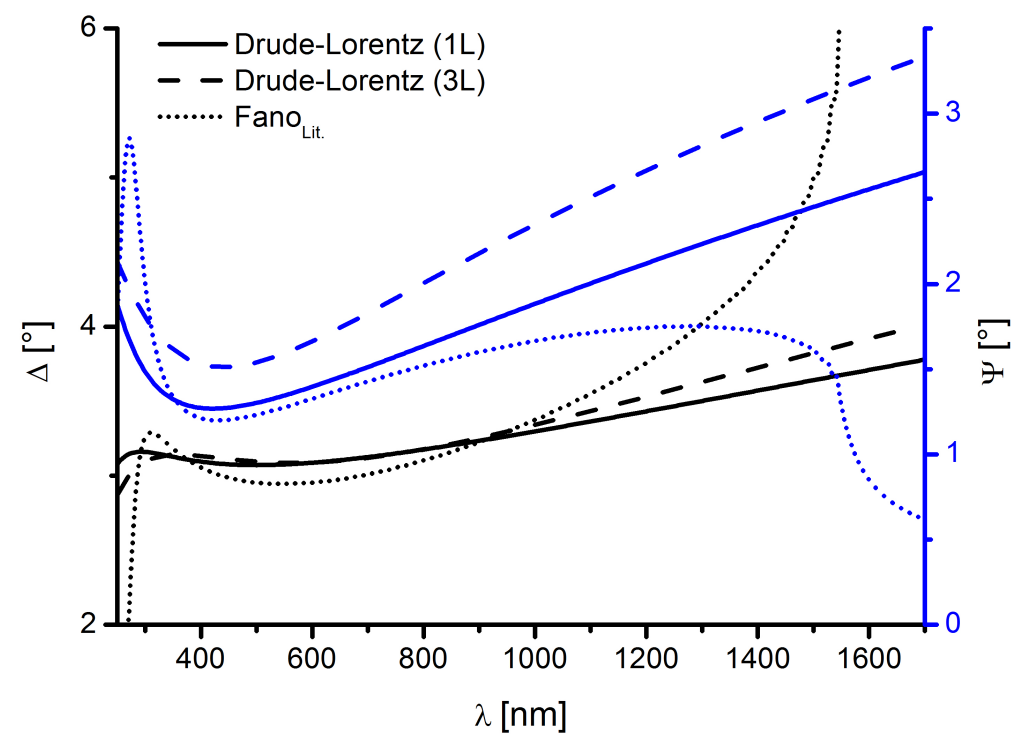

Abbildung 4.63 Vergleich der Dispersionen von Graphen modelliert mittels Fanoresonanz und Drude-Lorentz-Term.

Die Dispersion der Mono- und Trilage von Graphen sind im Vergleich zum FanoModel in Abbildung 4.63 gezeigt. Es ist deutlich zu erkennen, dass es außerhalb des UV/VIS-Bereichs zu deutlichen Abweichungen zwischen dem Fano-Model und dem Drude-Lorentz-Model kommt. Selbst die optischen Parameter einer Graphenmonolage unterscheidet sich von der Dispersion einer Trilage. So besitzt eine Trilage Graphen eine deutlich größere Absorption im NIR-Bereich, was einer stärkeren leitfähigkeit des Materials entspricht. An diesem Beispiel lässt sich gut erkennen, dass sich bei der Erweiterung des Wellenlängenbereichs erst neue Modelle etabliert und bislang bestehende überprüft werden müssen. 


\subsubsection{Spektroskopisches Mapping}

Bislang war das Messkonzept bei spektroskopischen Messungen so aufgebaut, dass im Vorfeld der Messung die gewünschten Bereiche mittels ROIs markiert wurden und das Signal jedes ROIs gemittelt aufgezeichnet wurde. Die Erweiterung der Software in mehreren Zonen automatisch spektroskopisch zu Mappen, erlaubt es dem Anwender sowohl gescannte als auch ungescannte Maps neben den numerischen Daten aufzunehmen. Erst bei der Auswertung der Daten entscheidet der Anwender, welche Bereiche zur Datenanalyse genutzt werden. Die Funktion des sogenannten Pixelshots (kleinster ROI) durch einen Stapel spektroskopisch aufgezeichneter Maps, sendet die entsprechenden Delta- und Psi-Daten ans Model zur Auswertung. Dabei können besonders kleine Strukturen bis hin zu einem Pixel ausgewertet werden.

Da besonders bei gescannten spektroskopischen Maps die Messzeit aufgrund des Scanningprozesses groß ist, wurde zusätzlich ein Tool entwickelt, einen möglichen Versatz der Maps zu korrigieren. Dieses Tool sorgt anhand von Kontrastbildern dafür, dass über die gesamte Messzeit sicher gestellt ist, dass es aufgrund von gerätespezifischen Bewegungen (wie beispielsweise dem Setzen des Goniometers) zu keinem Versatz der einzelnen Maps kommt. Der kompensierte Effekt tritt erst bei sehr hoher Auflösung (Pixel $=0.5 \mu \mathrm{m}$ ) auf und ist relevant bei Strukturen von $1.5 \mu \mathrm{m}$.

\section{Graphen}

An der im vorherigen Kapitel erwähnten Probe mit Graphenflocken auf SiliziumSubstrat mit einer $\mathrm{SiO}_{2}$ Schicht $(\mathrm{d}=292.3 \mathrm{~nm}$ ) wurden spektroskopische Maps (ungescannt) über den gesammten Wellenlängenbereich (250-1700 nm) aufgezeichnet. Die spektroskopischen Maps wurden unter Verwendung des 10x Objektivs und einem Einfallswinkel von $50^{\circ}$ aufgenommen. Ein Pixel der Map in x-Richtung entspricht umgerechnet $1 \mu \mathrm{m}$ auf der Probe.

Im oberen Teil der Abbildung ist im dem Kontrastbild der Probe ein Linienprofil (443 pixel) eingezeichnet, dessen spektroskopische Daten in den darunter folgenden Darstellungen gezeigt werden. Die mittleren 3D-Plots zeigen das Wellenlängenspektrum von Delta und Psi des Linienprofils. Um die sensitiven Bereiche zwischen Substrat und dem Graphen zu verdeutlichen, wurde von den Spektren ein Referenzspektrum (3. pixel) dividiert und die Differenz von Delta und Psi im unteren Teil der Abbildung visualisiert. Es ist deutlich zu erkennen, dass die größten Abweichungen in Delta und Psi bei den Wellenlängen erreicht werden, bei denen im spektralen Verlauf ein Maximum existiert (305 nm, $500 \mathrm{~nm}$ und $1500 \mathrm{~nm})$. Außerdem sind die Abweichungen zwischen einer Trilage und einer Monolage unterschiedlich. Somit wären Messungen bei 3 verschiedenen Wellenlängen ausreichend, um auf der Probe zwischen einer Mono- und Trilage zu differenzieren. Es sei jedoch erwähnt, dass diese Abweichungen zwischen Substrat und dem Graphen aus den optischen Eigenschaften sowie der Schichtdicke des $\mathrm{SiO}_{2}$ resultieren. Die Sensitivität bei $500 \mathrm{~nm}$ wird in der Literatur auch häufig genutzt, um mittels Lichtmikroskopie Graphenflocken zu betrachten.

$\mathrm{As}_{2} \mathrm{~S}_{3}$ 

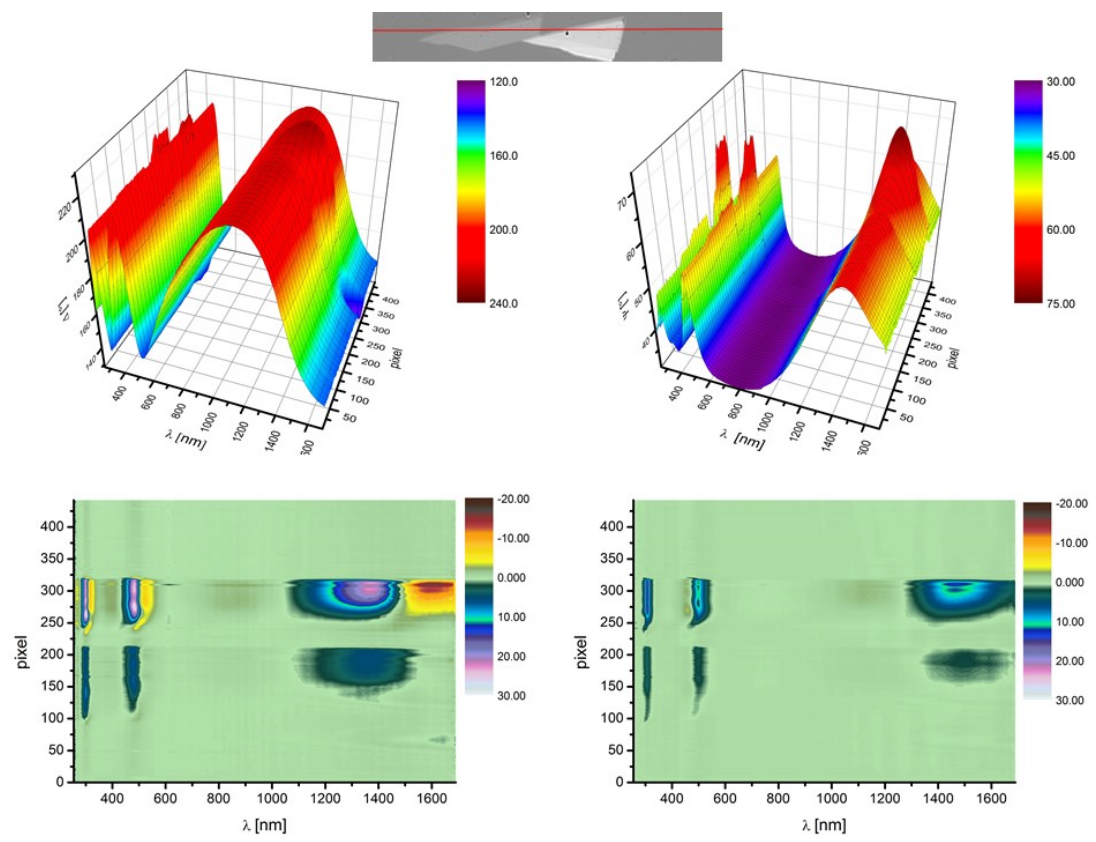

Abbildung 4.64 Übersicht der Messergebnisse eines Linienprofils spektroskopischer Maps von Graphen auf $\mathrm{Si}(100)$ mit $\mathrm{SiO}_{2}$ im UV/VIS/NIR-Bereich.

Auch an der $\mathrm{As}_{2} \mathrm{~S}_{3}$-Probe (siehe Kapitel 4.1.1.4) mit einer Schichtdicke von $960.5 \mathrm{~nm} \pm 0.1 \mathrm{~nm}$ wurden spektroskopische Maps im Bereich von 440-720 nm in $2 \mathrm{~nm}$ Intervallen aufgenommen. Die spektroskopischen Maps wurden an der Position mit idealer Belichtung unter Verwendung des 20x Objektivs und einem Einfallswinkel von 55 aufgenommen. Ein Pixel der Map in x-Richtung entspricht umgerechnet $0.7 \mu \mathrm{m}$ auf der Probe. Bei der Auswertung der Daten wurde analog zum vorherigen Probensystem ein horizontales Linienprofil (Länge $=76 \mu \mathrm{m}$ ) integriert (Abbildung 4.65, oben).

Im Kontrastbild der Probe ist das Linienprofil (109 pixel) eingezeichnet, dessen ellipsometrische Spektren in den darunter folgenden 3D-Plots gezeigt werden. Im unteren Teil der Abbildung 4.65 werden die Abweichungen von Delta und Psi bezogen auf das Referenzspektrum (3. pixel) visualisiert. Das Signal-Rausch-Verhältnis ist aufgrund des transparenten Substrats deutlich schlechter als im vorherigen Anwendungsfall und einer größeren lateralen Aufösung durch Verwendung des 20x Objektivs. Im unteren Teil der Abbildung ist das im Schichtmaterial projezierte holographische Gitter deutlicher zu erkennen. Auch hier sind die Abweichungen in Delta bzw. Psi bei den Wellenlänge am stärksten, dei denen das Wellenlängenspektrum ein Extrema besitzen. Wobei in diesem Beispiel Psi sensitiver ist und das holographische Gitter gut zu erkennen ist. Es handelt sich nicht um eine Variation der Schichtdicke des Materials, sondern um eine Varianz der optischen Parameter (Kapitel 4.1.1.4).

Beide Beispiele zeigen die maximale Informationsausbeute, die bei ellipsometrisch spektroskopischen Messungen mittels abbildender Ellipsometrie generiert werden können. Dazu wird eine maximale laterale Auflösung sowie die Sensitivtät der Ellipsometrie genutzt, um das Probensystem zu untersuchen. Da das Konzept des spektroskopischen Mappings vegleichsweise neu ist, steht bislang bei der Datenana- 

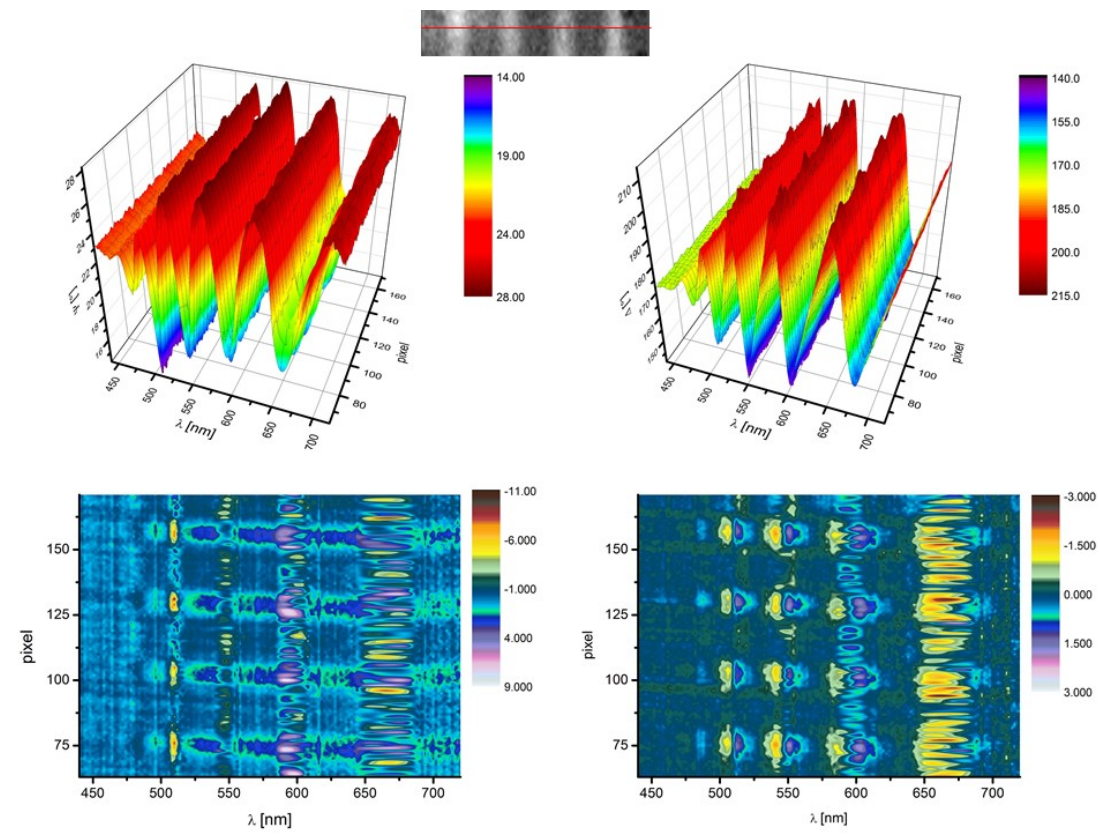

Abbildung 4.65 Übersicht der Messergebnisse eines Linienprofils spektroskopischer Maps von $\mathrm{As}_{2} \mathrm{~S}_{3}$ auf Glas im UV/VIS Bereich.

lyse (Ep4-Model) kein geeignetes Programm zur Verfügung. Besonders der Rechnungsprozess bezüglich der Parameter des jeweiligen Models und gleichzeitig der Schichtdicke ist noch nicht implementiert.

Durch das neues Messkonzept können im Nachhinein Daten ausgewählt und ausgewertet werden. Spektroskopische Maps enthalten zusätzlich Informationen über die Qualität der ausgewählten Bereiche, die bei rein nummerischen Daten nicht bekannt sind. 


\title{
4.3 Messablauf
}

Neben der Etablierung des spektroskopischen Mappings wurden ebenfalls andere Teilaspekte des Nullings verbessert. Neben der Anzahl der Iterationen sind ebenfalls Faktoren wie das Ausmass an Auslenkung der optischen Komponenten und Anzahl der gemessenen Zonen verantwortlich für die Messzeit. Diese Faktoren wurden beim abbildenden Ellipsometer untersucht und verbessert.

\subsubsection{Kompensator}

Betrachtet man die Bedingungen des Nullings (vgl. Gleichung 3.2), sollte der Kompensator exakt einem $\lambda / 4$-Plättchen entsprechen. Bei nur einer genutzten Wellenlänge lässt sich solch ein perfekter Kompensator realisieren. Für spektroskopische Messungen gelten die gleichen Bedingungen. In der Realität findet man kein optische Element, das über den gesamten spekktralen Bereich diese geforderte Eigenschaft erfüllt. Deshalb wurden bisher bei spektroskopischen Messungen 4 Zonen Messungen empfohlen, die solche gerätetechnischen Fehler durch Mitterlung korregieren. Diese sind jedoch sehr zeitaufwendig. Um die Inperfektion des Kompensators bei den spektroskopsichen Messungen zu berücksichtigen, wurde eine Art Korrektur in den Messablauf eingebaut. Dazu werden Charaktersitka des Kompensators wir retardierung und Achsenshift mittels spektralen 4 Zonen Messungen bestimmt und später bei der Messung mit einbezogen.
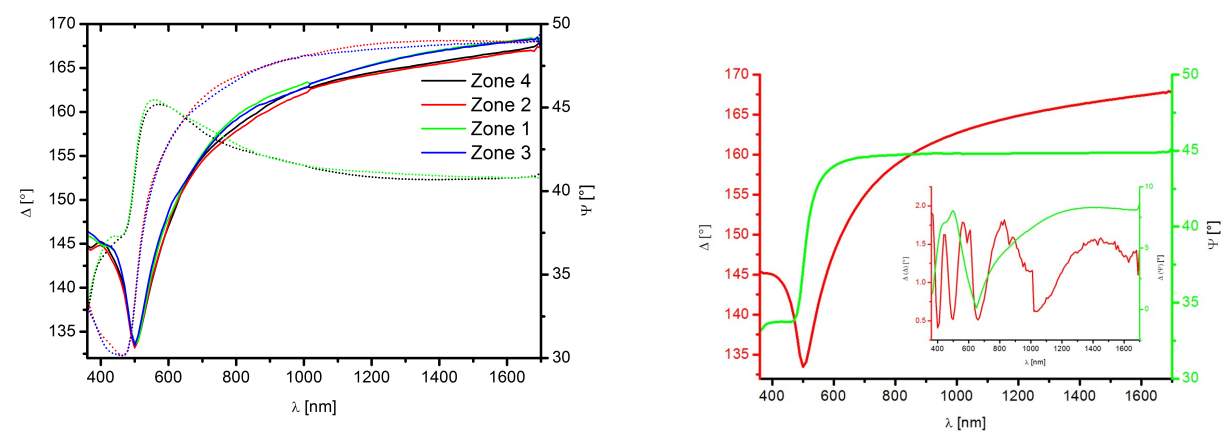

\begin{abstract}
Abbildung 4.66 Spektroskopische Messung von 360-1700 nm (4 Zonen) an intransparenter Goldoberfläche ohne Korrektur des Kompensators und Mittelwerte bzw. Abweichungen von Delta und Psi aus den 4 Zonen.
\end{abstract}

Auf der linken Seite der Abbildung 4.66 werden die 4 Zonen einer spektroskopische Messung von 360-1700 $\mathrm{nm}$ bei einem Einfallswinkel von $50^{\circ}$ an einer intransparenten Goldoberfläche gezeigt. Die Ergebnisse einer Mittelung der 4 Zonen sowie die mittlere Abweichung der Zonen ist auf der rechten Seite der Abbildung 4.66 graphisch dargestellt. Anhand der unkorrigierten Daten wird die Retardierung und Achsenshift des Kompensators iterativ berechnet. Der wellenabhängige Verlauf der Charakteristika des Kompenators sind in Abbildung 4.67 gezeigt. 

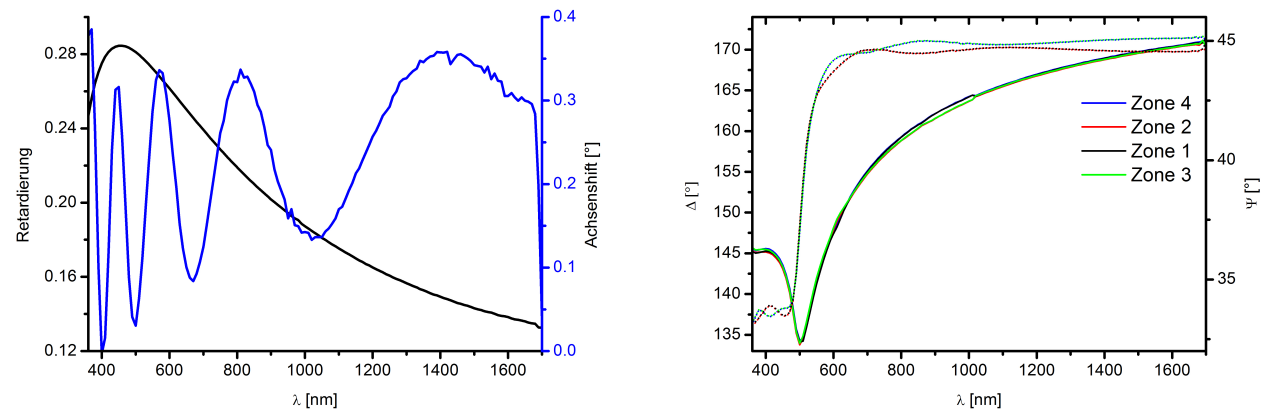

\begin{abstract}
Abbildung 4.67 Retardierung und Achsenshift des Kompensators und spektrokopische 4 Zonen Messung mit Kompensatorkorrektur.
\end{abstract}

Werden die beiden Charakteristika des Kompensators bei der spektroskopsichen Messung einbezogen, erhält man bei Messungen (2 Zonen) die gleiche Qualität wie bei 4 Zonen (Abbildung 4.67, rechts), wodurch sich die Messzeit sich halbieren lässt.

Die Charakterisierung des Kompensators lieferte einen Anknüpfungspunkt, um das Gerät auch im Rotating Compensator Modus zu betreiben. So könnten besonders inhomogene Proben (Schichtdickenvarianz > $100 \mathrm{~nm}$ ) mit abbildender Ellipsometrie untersucht werden. Die standardmäßige Implementierung in die Bedienungssoftware steht noch aus.

\title{
4.3.2 Fit
}

Bei der Datenanalyse wird neben dem RMSE-Wert (vgl. Gleichung 3.4) zusätzlich für jeden Datenpunkt die Abweichung der gemessenen Delta- bzw. Psi-Werte zum modellierten Wert gezeigt. Dabei erhielt man bei spektroskopischen Messungen mit nur einer Iteration oder großen Schrittweiten Abweichungen der simulierten zu den experimentellen Daten, die dem Verlauf des Spektrums ähneln. Zu Beginn dieser Arbeit wurden beim Nulling das Intensitätsminimum anhand der Polarisator- bzw. Analysatorstellung durch Regression einer Parabel bestimmt. Große Auslenkungen der optischen Komponenten oder Startpunkte der optischen Kompenten fern der Nulling Position, zeigen jedoch eine deutliche Abweichung des parabolischen Verhaltens bei der Aufzeichnung der Intensitätskurve. Dabei kommt es zu einem Fehler beispielsweise bei der Schichtdickenbestimmung von $\mathrm{SiO}_{2}$ (siehe Tabelle 4.14). Bei der Messung wurde eine homogene Probe im Wellenbereich von 380-800 nm (20 nm Intervalle) spektroskopisch mittels abbildender Ellipsometrie untersucht.

Dabei ist deutlich zu erkennen, dass es bei der Probe mit $\mathrm{SiO}_{2}$ auf Silizium-Substrat besonders zu Abweichungen in der berechneten Schichtdicke kommt, wenn beim Nulling-Prozess große ranges eingestellt werden. Die maximale Anzahl von drei Iterationen reicht bei beiden Fits aus, um die Ist-Schichtdicke zu bestimmen.

Um den Einfluss des Fits, sowie den Einfluss zwischen traditionellen und dem neu etablierten Ep4-Nullings zu ermitteln, wurde eine Probe (Abbildung 4.68) mit vier 


\begin{tabular}{|c||c|c|c|c|c|c|c|c|c|}
\hline \multicolumn{1}{|c||}{ ranges } & \multicolumn{3}{c|}{10} & \multicolumn{3}{c|}{25} & \multicolumn{3}{c|}{40} \\
\hline Iterationen & 1 & 3 & 5 & 1 & 3 & 5 & 1 & 3 & 5 \\
\hline Parabel & 291.8 & 291.7 & 291.7 & 292.1 & 291.7 & 291.7 & 292.4 & 291.7 & 291.7 \\
\hline Kosinus & 291.8 & 219.7 & 219.7 & 291.9 & 219.7 & 219.7 & 219.7 & 219.7 & 219.7 \\
\hline
\end{tabular}

Tabelle 4.14 Einfluss der Auslenkung (ranges) der optischen Komponenten auf die Bestimmung der Schichtdicke bei spektroskopischen Messungen.

verschiedenen $\mathrm{SiO}_{2}$-Schichtdicken auf Silizium-Substrat bei 40 ranges vermessen. Im Vergleich zum traditionellen Nulling, bei dem die Ist-Position der jeweils fixen optischen Komponente berücksichtigt wird, wird beim Ep4-Nulling die bestimmte Nullposition der optischen Komponenten in Bezug zur Position der anderen Komponente gesetzt. Nach Anwendung eines internen Algorythmus ergibt sich die absolute Minimumpositionen.

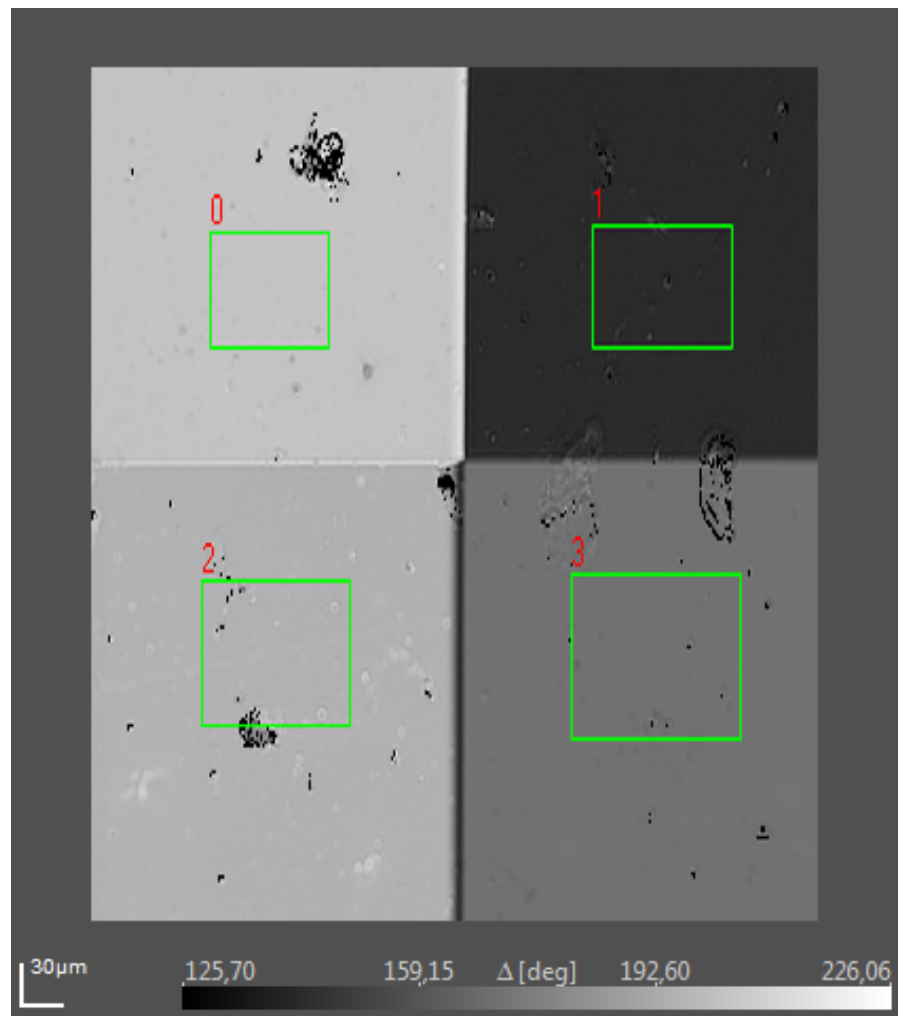

Abbildung 4.68 Delta-Map einer Probe mit vier Messbereichen verschiedener $\mathrm{SiO}_{2}$-Schichtdicken auf $\mathrm{Si}$ (100) bei $500 \mathrm{~nm}$.

Dazu wurden spektroskopische Messungen im Wellenlängenbereich von 380-800 nm in $20 \mathrm{~nm}$ Intervallen durchgeführt. Alle vier Areale wurden gleichzeitig und bei einem Einfallswinkel von $50^{\circ}$ mit einem 10x Objektiv gemessen. Anschließend wurden die Schichtdicken unter Einbezug der optischen Parameter der Materialien aus der Datenbank bestimmt. Neben einer hohen range der optischen Komponente von $40^{\circ}$ wurde eine Anzahl an Iterationen von 1 und 3 gewählt. Tabelle 4.15 zeigt die 
ermittelten Schichtdicken für die spektroskopische Messung mittels traditionellem Nulling, inklusive Fehler und RMSE-Wert.

\begin{tabular}{|c|c|c|c|c|c|c|}
\hline Fit & It. & & ROI 0 & ROI 1 & ROI 2 & ROI 3 \\
\hline \multirow{4}{*}{ Parabel } & \multirow{2}{*}{1} & $\mathrm{~d}\left(\mathrm{SiO}_{2}\right)[\mathrm{nm}]$ & $161.8 \pm 0.5$ & $71.5 \pm 0.3$ & $106.7 \pm 0.2$ & $14.2 \pm 0.2$ \\
\hline & & RMSE & 5.167 & 2.815 & 2.481 & 1.312 \\
\hline & \multirow{2}{*}{3} & $\mathrm{~d}\left(\mathrm{SiO}_{2}\right)[\mathrm{nm}]$ & $161.7 \pm 0.5$ & $71.8 \pm 0.3$ & $106.7 \pm 0.2$ & $14.3 \pm 0.2$ \\
\hline & & RMSE & 5.236 & 2.781 & 2.368 & 1.382 \\
\hline \multirow{4}{*}{ Kosinus } & \multirow{2}{*}{1} & $\mathrm{~d}\left(\mathrm{SiO}_{2}\right)[\mathrm{nm}]$ & $162.1 \pm 0.5$ & $71.6 \pm 0.2$ & $106.9 \pm 0.1$ & $14.2 \pm 0.2$ \\
\hline & & RMSE & 5.041 & 2.200 & 1.827 & 0.677 \\
\hline & \multirow{2}{*}{3} & $\mathrm{~d}\left(\mathrm{SiO}_{2}\right)[\mathrm{nm}]$ & $161.9 \pm 0.5$ & $71.7 \pm 0.2$ & $106.9 \pm 0.1$ & $14.2 \pm 0.2$ \\
\hline & & RMSE & 5.075 & 2.128 & 1.791 & 0.716 \\
\hline
\end{tabular}

Tabelle 4.15 Fit-Ergebnisse der $\mathrm{SiO}_{2}$-Probe unter Verwendung des traditionellen Nullings.

Die simultane Bestimmung der Schichtdicke mittels wellenlängenabhängiger Ellipsometrie zeigt bei der Verwendung des traditionellen Messvorgangs keine deutliche Minimierung des RMSE-Wertes - als Indiz für die Qualität der Messung -, wenn bei der Messung der Fit oder die Iterationanzahl variiert werden. Prinzipiell sind die RMSE-Werte groß, im Vergleich zum angestrebten Idealwert von 1. Erst durch Verwendung des Ep4-Nullings (Tabelle 4.16) zeigt sich eine deutlichere Übereinstimmung zwischen den generierten Datenwerten und dem simulierten Spektrum.

\begin{tabular}{|c|c|c|c|c|c|c|}
\hline Fit & It. & & ROI 0 & ROI 1 & ROI 2 & ROI 3 \\
\hline \multirow{4}{*}{ Parabel } & \multirow{2}{*}{1} & $\mathrm{~d}\left(\mathrm{SiO}_{2}\right)[\mathrm{nm}]$ & $162 \pm 0.3$ & $\overline{71.8 \pm 0.2}$ & $106.8 \pm 0.1$ & $14.3 \pm 0.3$ \\
\hline & & RMSE & 3.207 & 2.063 & 1.901 & 1.569 \\
\hline & \multirow{2}{*}{3} & $\mathrm{~d}\left(\mathrm{SiO}_{2}\right)[\mathrm{nm}]$ & $161.9 \pm 0.3$ & $71.8 \pm 0.2$ & $106.8 \pm 0.1$ & $14.4 \pm 0.3$ \\
\hline & & RMSE & 3.208 & 2.028 & 1.914 & 1.597 \\
\hline \multirow{4}{*}{ Kosinus } & \multirow{2}{*}{1} & $\mathrm{~d}\left(\mathrm{SiO}_{2}\right)[\mathrm{nm}]$ & $162.3 \pm 0.1$ & $71.8 \pm 0.1$ & $107 \pm 0.1$ & $14.4 \pm 0.2$ \\
\hline & & RMSE & 1.006 & 0.386 & 0.608 & 0.374 \\
\hline & \multirow{2}{*}{3} & $\mathrm{~d}\left(\mathrm{SiO}_{2}\right)[\mathrm{nm}]$ & $162.3 \pm 0.1$ & $71.7 \pm 0.1$ & $107 \pm 0.1$ & $14.3 \pm 0.2$ \\
\hline & & RMSE & 0.968 & 0.392 & 0.555 & 0.414 \\
\hline
\end{tabular}

Tabelle 4.16 Fit-Ergebnisse der $\mathrm{SiO}_{2}$-Probe unter Verwendung des Ep4-Nullings.

Eine Erhöhung der Iterationanzahl führt nicht zu einer Minderung des RMSEWertes. Die Fit-Analyse unter Verwendung der Kosinusfunktion führt bei großen Auslenkungen von Polaristor und Analystor zu einer deutlichen Verringerung des RMSE-Wertes, was einer exakteren Bestimmung der ellipsometrischen Parameter entspricht. Dabei werden, unabhängig von der Iteration, RMSE-Werte um 1 und kleiner vom Model berechnet.

Zusammenfassend lässt sich festhalten, dass der bisher angewendete Parabel-Fit zu leichten Abweichungen in der Bestimmung der absoluten Minimumposition kommt, wenn bei den Messungen große ranges genutzt werden. 
Neben der Qualität der Messwerte steht besonders die aufgewendete Messzeit im Vordergrund, die durch Halbierung der Anzahl der Zonen auf 2 und der Iterationen auf 1 erreicht wird und etwa nur noch einem Viertel der bisherigen Zeit entspricht. 


\subsection{Zellen}

Bei in-situ-Messungen werden Ellipsometer in Kombination mit Zellen genutzt. Die Messungen in dieser Arbeit wurden überwiegend ex-situ durchgeführt - und zwar an der Grenzfläche zwischen Probe und Luft unter Standardbedingungen (Atmosphärendruck, Umgebungstemperatur $\left(20^{\circ} \mathrm{C}\right)$ ). Durch die Verwendung von Zellen können physikalische Parameter wie Temperatur oder Luftfeuchtigkeit variiert werden. Bei geschlossenen Systemen können die Grenzflächen zwischen der Probe und Flüssigkeiten (fest-flüssig (solid-liquid, SL)) bzw. invers (flüssig-fest (liquid-solid, LS)) betrachtet werden. Zellen, wie die SL-Zelle, sind messtechnisch auf einen Einfallswinkel begrenzt, da ein orthogonaler Einfall des Lichtes erforderlich ist, um beim Übergang ins wässrige Medium eine Brechung des einfallenden Lichtkegels zu vermeiden. Bei Zellen, die im Kretschmann-Aufbau verwendet werden, ist die Limitierung des Einfallswinkels aufgehoben. Bei nicht orthogonalem Einfallswinkel entspricht der externe Einfallswinkel nicht dem internen Winkel, der unter Einbezug der optischen Parameter berechnet werden kann. In dieser Arbeit wurden zwei neue in-situ-Systeme mit dem abbildenen Ellipsometer kombiniert und an den jeweiligen Probensystemen getestet.

\subsubsection{Temperaturzelle}

Neben den klassischen Phasenübergängen zeigen amorphe Materialien wie Polymere und Lipide temperaturabhängige Charakteristika. Bei der Glastemperatur Tg kommt es zu einem Übergang von der harten glasartigen zur zähflüssigen gummiartigen Phase. Dabei bestimmt die Struktur des Moleküls die Glastemperatur. Häufig wird die dynamische Differenzkalorimetrie (DSC) als thermische Analysemethode eingesetzt, um die Lage des Übergangs zu bestimmen. Bei dünnen Schichten wird häufig die temperaturabhängige Ellipsometrie eingesetzt, da beim Phasenübergang eine Änderung der optischen Parameter zu beobachten ist. Aufgrund der Sensitivität lässt sich die Phasenübergangstemperatur von dünnen amorphen Materialien bestimmen. Im Rahmen der Dissertation wurde eine modifizierte Heizzelle (LinkAm, TM50) an das Gerät adaptiert. Die Zelle besteht prinzipiell aus einem Heizblock, der zusätzlich mittels flüssigem Stickstoff gekühlt werden kann, um eine Linearität der Temperatur bei Kühl- und Heiz-Zyklen zu gewährleisten. Die Zelle besitzt zusätzlich eine Abdeckung, um sie evakuieren zu können und so einen Wärmeaustausch zwischen Probe und Umgebung zu verhindern. Die Abdeckung der Zelle wurde bei den Messungen entfernt, da aufgrund des Arbeitsabstandes der Objektive keine Messungen im geschlossenen System möglich waren. Aus sicherheitstechnischen Gründen wurde auf eine Kühlung des Heizblocks durch flüssigen Stickstoff verzichtet. Die Kühlung der Probe erfolgte durch den Wärmeaustausch mit der Atmosphäre. Die temperaturabhängigen Messungen wurden bei einem Einfallswinkel von $60^{\circ}$ und unter Verwendung des 10x Objektivs durchgeführt. Als Lichtquelle wurde der Laser genutzt.

In Abbildung 4.69 wird die graphische Bestimmung der Glasübergangstemperatur von Polystyrol auf Silizium-Substrat anhand des temperaturabhängigen Verlaufs des 


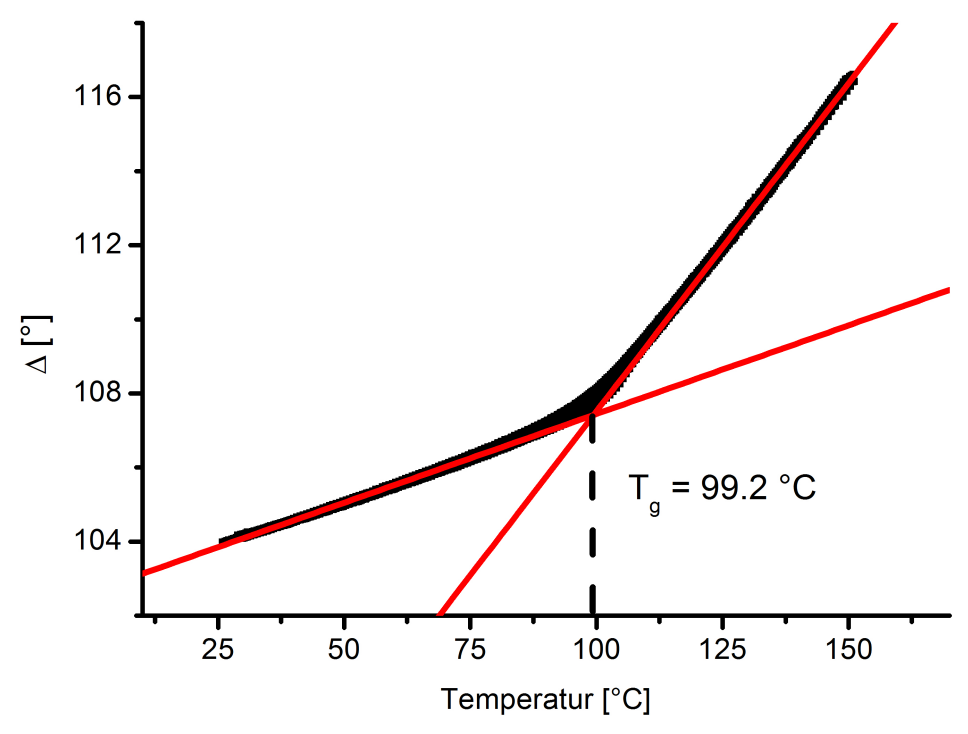

Abbildung 4.69 Glasübergangstemperatur von Polystyrol $(\mathrm{d}=112 \mathrm{~nm})$.

sensitiven Parameters Delta gezeigt. Bei der Messung wurden fünf Temperaturrampen bei variiernden Heiz- bzw. Kühlraten gefahren. Die Datenpunkte fallen übereinander, so dass der Glasübergang von Polystyrol unabhängig von der Heiz- bzw. Kühlrate bestimmt werden konnte. Die Schichtdicke wurde zuvor bei $20^{\circ} \mathrm{C}$ durch spektroskopische Ellipsometrie bestimmt. Der Wert für die Glasübergangstempera$\operatorname{tur}\left(\mathrm{T}_{\mathrm{g}}=99.2^{\circ} \mathrm{C}\right)$ stimmt mit dem aus der Literatur überein [13].

Zusätzlich wurde mit der Heizzelle und unter Verwendung der abbildenden Ellipsometrie die Zersetzungstemperatur (Abbildung 4.70) von Polystyrol auf SiliziumSubstraten bestimmt.

Bei den Proben handelt es sich um homogene dünne Filme, durch die abbildende Funktion lassen sich ebenfalls mikrosturkturierte polymere Filme untersuchen. Da die Temperaturänderung die optischen Eigenschaften des Substrats beeinflusst, wurde auf eine Umrechnung in Schichtdicke bzw. Brechungsindex des Polystyrols verzichtet. Um die Temperaturabhängigkeit des Substrates mit einzubeziehen, würde sich eine Probe empfehlen, bei der neben dem polymeren Film zugleich das blanke Substrat vermessen wird. Diese Option steht jedoch nur bei der Verwendung eines abbildenden Ellipsometers zur Verfügung 


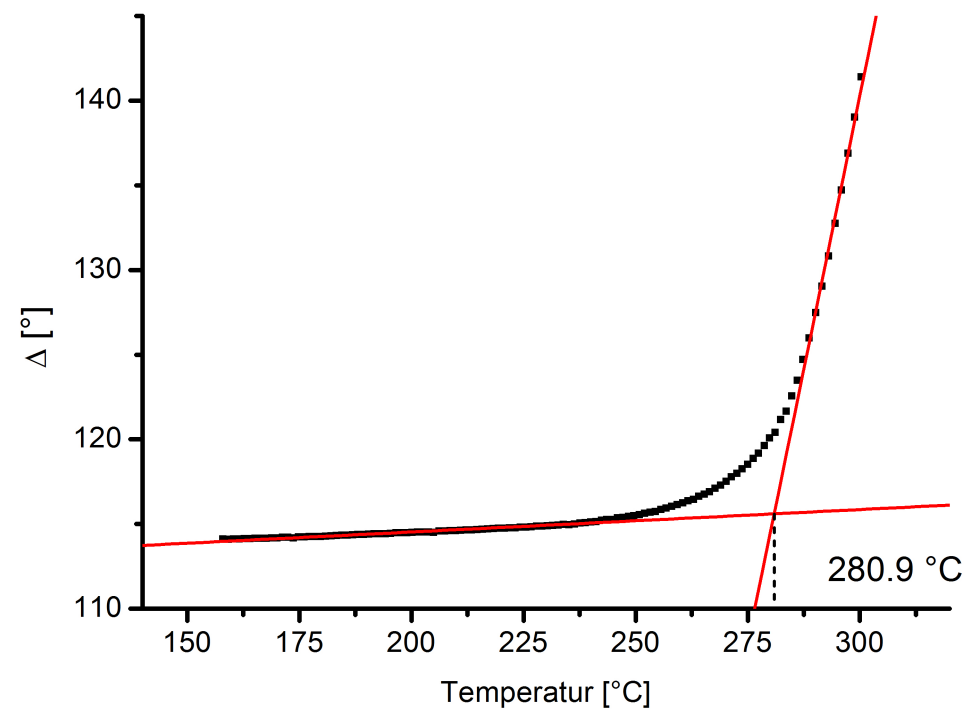

Abbildung 4.70 Zersetzungstemperatur von Polystyrol ( $\mathrm{d}=54$ $\mathrm{nm})$.

\subsubsection{Light-guides}

Neben den klassischen Grenzflächen fest-gasförmig, fest-flüssig und flüssig-gasförmig ist besonders für bipolare Reagenzien die Untersuchung an der flüssig-flüssig Grenzfläche von Bedeutung. Dabei werden neben der wässrigen Phasen unpolare Flüssigkeiten wie Öl oder Toluol genutzt. In dieser Arbeit wurden unter Verwendung von Light-guides Messungen an der flüssig-flüssig-Grenzfläche durchgeführt. Dabei sind wie in Abbildung 4.71 gezeigt, die Light-guides am Laserarm bzw. Objektiv befestigt. 


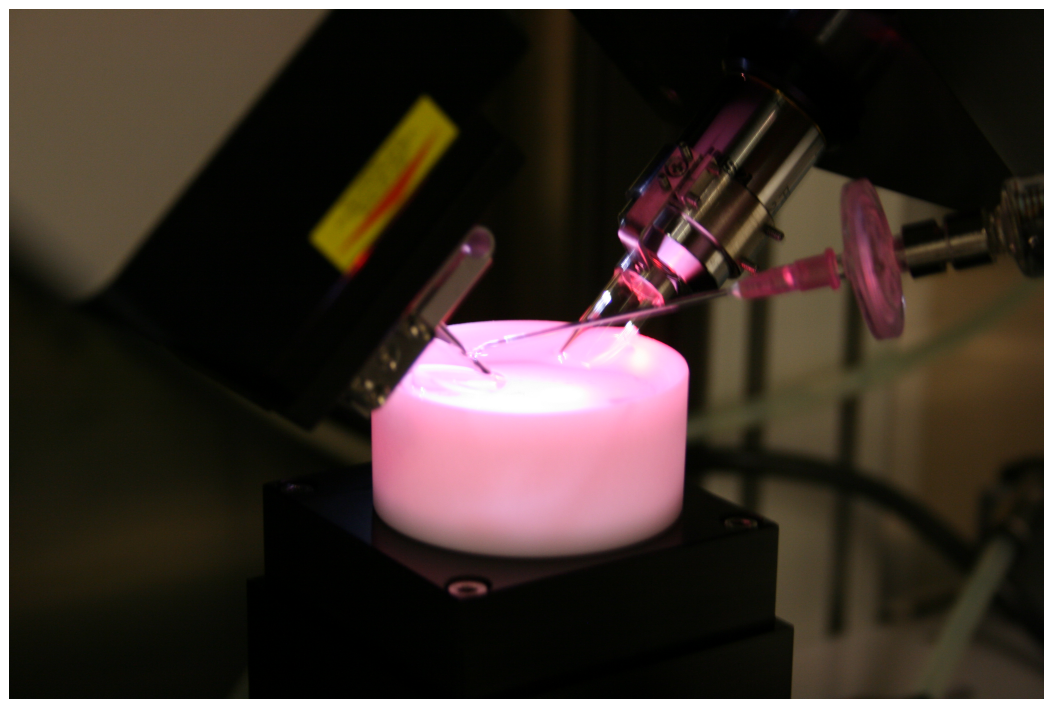

Abbildung 4.71 Light-guides bei Messung an der flüssig-flüssigGrenzfläche [1].

Unabhängig vom Einfallswinkel ist ein orthogonales Treffen des Lichtkegels ins wässrige Medium gewährleistet. Die Ligth-guides werden vor der Messung kalibriert und justiert um eine Doppelbrechung bzw. einen Polarisationseffekt ausschließen zu können. Als Gefäß wird ein runder Teflontrog genutzt, in dem sich am Grund eine Blackplate befindet, um Streulicht zu vermeiden. Da es sich beim Trog um ein offenes System handelt, wurde das häufig verwendete leicht flüchtige Toluol durch ein farbloses Feinöl ersetzt.

Zunächst wurde der geeignete Einfallswinkel für die Parafinöl/Wasser-Grenzfläche durch ellipsometrische Winkelspektren bestimmt. Die Ergebnisse des AOI-Spektrums (4 Zonen, $40-44^{\circ}, 0.2^{\circ}$ Intervalle) mit dem $5 x$ Objektiv und dem breitbandigen Laser $(\lambda=658 \mathrm{~nm})$ sind in Abbildung 4.72 gezeigt. 


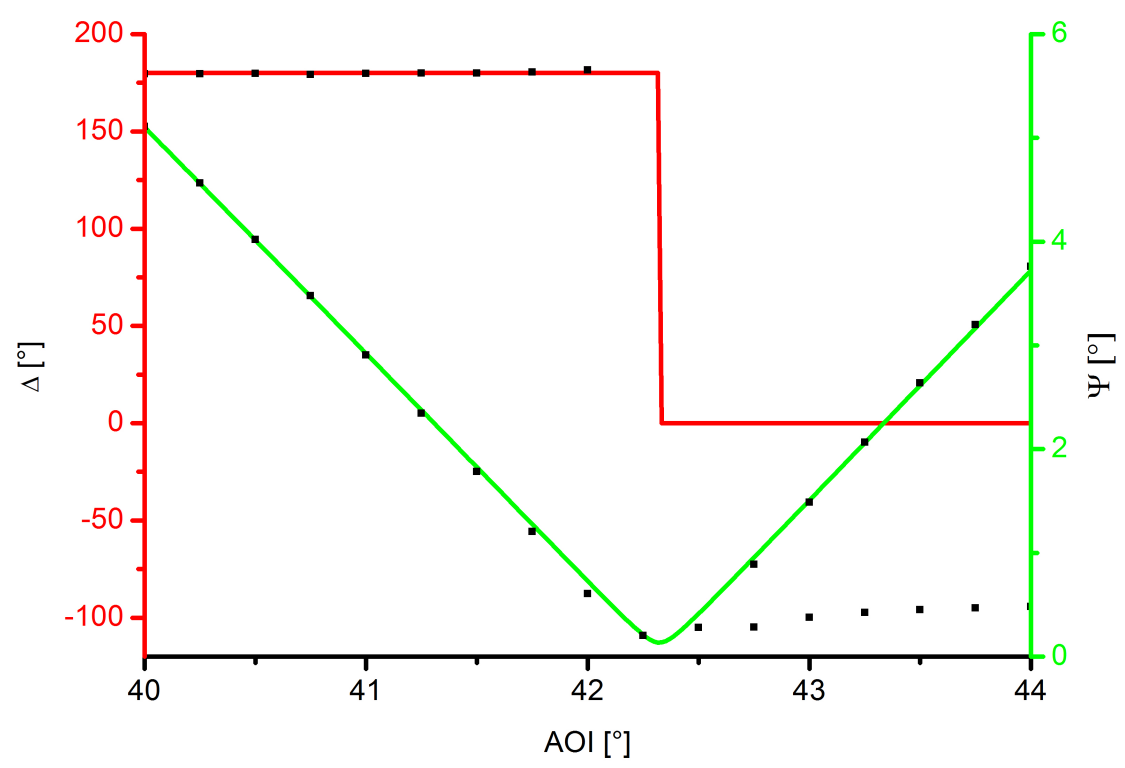

Abbildung 4.72 AOI-Spektrum an der Parafinöl/WasserGrenzfläche bei einer Wellenlänge von $658 \mathrm{~nm}$.

Bei der Datenanalyse wurde im Model das Feinöl als transparentes Material mit Hilfe von $\mathrm{n}+\mathrm{k}$-fix modelliert. Die optischen Parameter für Wasser bei $20^{\circ} \mathrm{C}$ entstammen aus der Datenbank. Bei der Modellierung zeigte sich besonders Psi deutlich sensitiver als Delta, weshalb zunächst nur die Psi-Werte zur Bestimmung von n berücksichtigt wurden.

Am gleichen System wurde bei einem festen Winkel von $40^{\circ}$ der Spektralbereich $\lambda=450-850 \mathrm{~nm}$ in vier Zonen vermessen. Als Model wurde unter Einbeziehung der Dispersion von Wasser aus der Datenbank für das Parafinöl ein Cauchy-Term verwendet. Die simulierten Daten für Psi entsprachen sehr gut dem realen System. In Delta zeigen sich jedoch noch deutliche Abweichungen. Im nächsten Schritt wurde eine Grenzschicht zwischen Wasser und Öl angenommen $(\mathrm{d}=0.37 \pm 0.04 \mathrm{~nm})$, die als Gemisch aus Wasser und Luft modelliert wurde (Bruggemann). Die Schichtdicke dieser Grenzschicht wurde neben den Cauchy-Koeffizienten als Fit-Parameter frei gegeben. Die Ergebnisse der Modellierung sowie die sich ergebene Dispersion für das Parafinöl sind in Abbildung 4.73 gezeigt. 

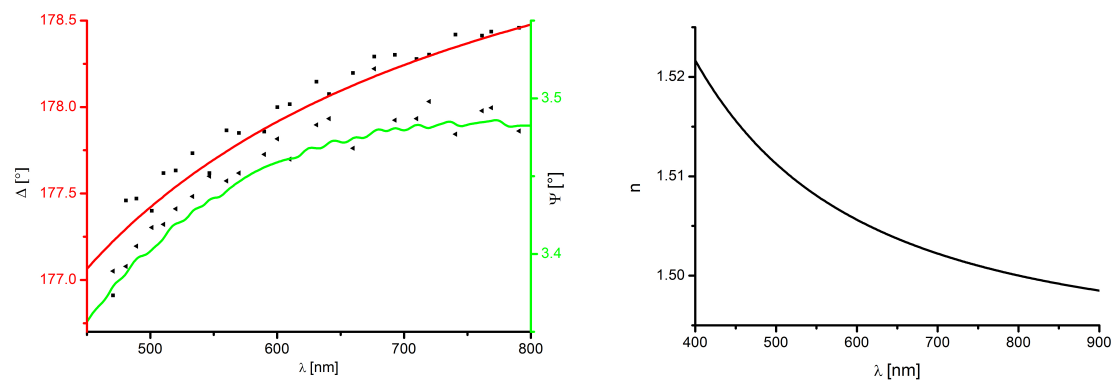

Abbildung 4.73 Delta/Psi-Wellenlängenspektrum an der Parafinöl/Wasser-Grenzfläche bei einem Einfallswinkel von $40^{\circ}$.

Nachdem $20 \mu \mathrm{L}$ einer Lösung an Polystyrol in Touol $(2.5 \mathrm{~g} / \mathrm{mL})$ an die Grenzfläche gegeben wurde, wurde nach dem Entweichen des Lösungsmittels analoge spektrokopsiche Messungen durchgeführt. Zusätzlich wurden Kontrast-Bilder und Maps bei bestimmten Wellenlängen des Polystyrolfilms aufgenommen.
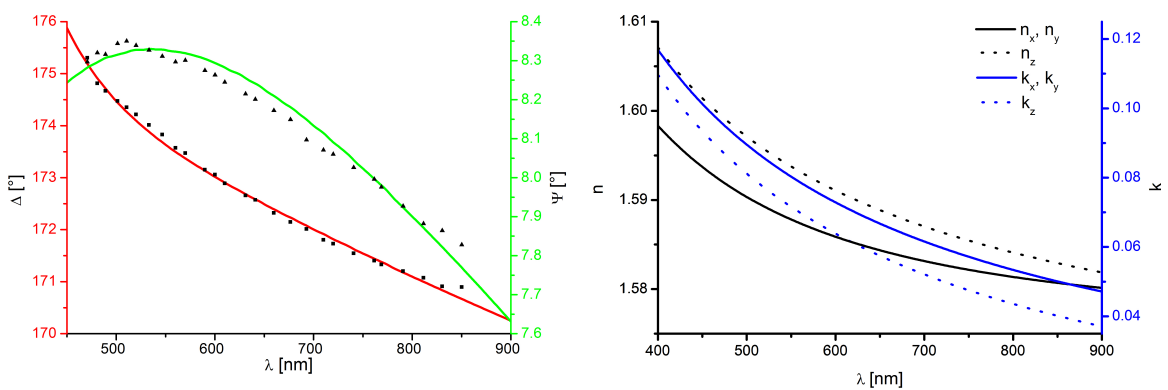

\begin{abstract}
Abbildung $\quad \mathbf{4 . 7 4}$ Delta/Psi-Wellenlängenspektrum eines anisotropen Polystyrolfilms (Dispersion, rechts) an der Parafinöl/Wasser-Grenzfläche bei einem Einfallswinkel von $40^{\circ}$.
\end{abstract}

Die Auswertung der Wellenlängenspektren der ROI basierenden Messung ergaben eine Schichtdicke von $120.4 \pm 2.6 \mathrm{~nm}$ für den Polystyrolfilm. Als Model wurden das optische Verhalten mit einer Anisotropie in der z-Ebene aus jeweils einem LorentzTerm beschrieben. Wobei der Brechungsindex unter dem der Literatur liegt [34]. Dieser Effekt lässt sich jedoch durch das Feinöl erklären, da es sich nicht um reines Polystyrol halten wird und es zu einer Mischung der Brechungsindizes beides Substanzen kommt. Im Gegensatz zu Polystyrol als bulk Material zeigen die modllierten optischen Parameter des Films eine leichte Absorption, wobei es sich um eine pseudo-Absoprtion halten könnte, die durch Streuzentren wie feine Luftblässchen im Polystyrol verursacht werden können. In Abbildung 4.75 sind die Psi-Map des Polystyrolfilms bei $658 \mathrm{~nm}$ und die basierend auf dem Model umgerechnete Schichtdickenkarte des Films an der Öl/Wasser-Grenzfläche gezeigt. Das Polystyrol bildet an der Grenzfläche zwischen dem Feinöl und Wasser einen homogenen Film aus. Dabei zeigt der Polystyrolfilm einen leichten Gradienten beim Übergang in den nicht benetzten Bereich an der Grenzfläche. 

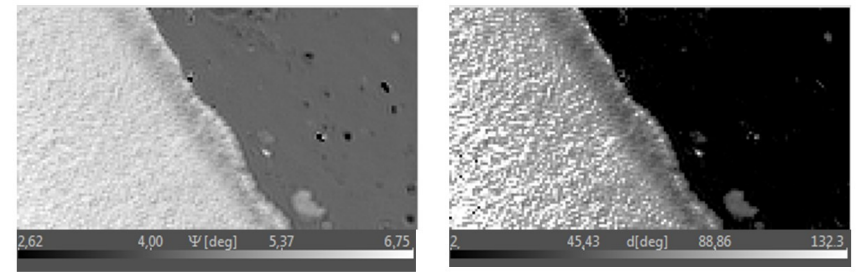

Abbildung 4.75 Psi- und Schichtdickenkarte eines PS-Films an der Öl/Wasser-Grenzfläche bei $658 \mathrm{~nm}$.

Durch Kombination von LB-Equipement für flüssig-flüssig-Grenzflächen ist es in Zukunft möglich spektroskopische Untersuchungen mittels abbildender Ellipsometrie durchzuführen. 


\section{Zusammenfassung und Ausblick}

Im Rahmen der angefertigten Arbeit konnte gezeigt werden, in wie weit die abbildende Ellipsometrie die Vorteile von Bildgebung, Ellipsometrie und Spektroskopie vereint. Neben den quantitativen und spektroskopischen Messungen konnten die gravierenden Vorteile der Messtechnik beim spektroskopischen Mapping demonstriert werden. Selbst atomare Schichten oder die minimale Varianz der Dispersion können detektiert werden und sind im Bereich der Erforschung von 2D-Materialien zukunftsweisend. Die laterale Auflösung ist zwar limitiert, die Auflösung in z-Richtung ist vergleichbar mit Aufnahmen von AFMs. Für die gesamtheitliche Nutzung der Technik werden zukünftig spektroskopische Maps im Vordergrund stehen, wobei die spektralen Maps mittels Alignment-Tool im Nachhinein korrigiert werden können. Mit Hilfe dieses Tools und kleineren Änderungen im Messablauf wäre es sogar möglich, bessere ellipsometrische Aufnahmen von Monolagen an der Luft-Wasser-Grenzfläche zu generieren, was bisher nicht umgesetzt worden ist.

Der bisherige negativ Faktor Messzeit konnte durch geeignete Korrekturen des Kompensators und Reduzierung der Iterationen deutlich verbessert werden. Durch Einführung des Ultra-Objektivs gelingt es, in einem bestimmten Winkelbereich ein komplett fokussiertes Bild zu generieren, jedoch wird durch die Scheinflugoptik eine schlechtere Auflösung im Vergleich zur Scanner Option erreicht. Die abbildende Ellipsometrie wird jedoch nie die Messgeschwindigkeit von nicht bildgebenden Ellipsometern erreichen.

Die Erweiterung des Wellenlängenbereichs ins UV bzw. NIR ermöglicht neue Anwendungsfelder für ellipsometrische Untersuchungen von dünnen Mikrostrukturen, deren Absorption in den jeweiligen Bereichen liegen. Somit wäre es möglich DNASpots auf herkömmlichem Glas im UV-Bereich mittels abbildender Ellipsometrie zu visualieren und so auf Marker, wie bei der Floureszenzmikroskopie, zu verzichten.

In dieser Arbeit konnten erstmals dünne, transparente, anisotrope Folien als Substrat unter Verwendung des Beamcutters gemessen werden. Wobei generell die Nutzung des Beamcutters ein zentraler Aspekt bei Messungen von transparenten Substraten mittels Ellipsometrie darstellt. Dabei sei besonders erwähnt, dass Substratdicken unter $50 \mu \mathrm{m}$ schwierig zu vermessen sind.

Durch die Etablierung des Monochromators bei der Gerätegeneration Ep4 können sowohl Schichtdicken größer $5 \mu \mathrm{m}$ gemessen als auch optische Charakteristika wie Absorptionsbanden und Bandlücken des Filmmaterials exakt bestimmt werden. Die Berücksichtigung der Bandbreite der genutzten Wellenlänge seitens des Ep4Models zeigt eine deutliche Verbesserung beim Fitting Prozess. Besonders bei SPRMessungen wurde bis dato keine Bandbreite berücksichtigt, was zu Abweichungen der Simulation zu den aufgenommenen Messwerten führte. Aufgrund der Durch- 
stimmbarkeit der Wellenlänge können Messungen aufgrund der größeren Anzahl an Messpunkten deutlich länger dauern.

Die Automatisierung des Messvorgangs und die Erweiterung der Software seitens der Datenanalyse und Darstellung von spektralen Maps sind zentrale Themen in der Forschung und Entwicklung der Firma Accurion GmbH. Dabei können die hohe Anzahl an Möglichkeiten und die Komplexität der Messtechnik den neuen Anwender anfänglich überfordern. An dieser Stelle ist ein deutlicher Optimierungsbedarf seitens des Anwender erkennbar, da bisher vorwiegend Universitäten oder andere Forschungseinrichtungen diese Messtechnik nutzen.

In dieser Arbeit wurde die Vielseitigkeit der Messtechnik gezeigt und es konnten erstmals mikrostrukturierte biaxial anisotrope Materialen charakterisiert werden. Mit Hilfe der Drehung der Probe bei p- oder s-polarisiertem einfallenden Licht kann schnell geprüft werden, ob es sich um eine isotrope oder anisotrope Probe handelt. Diese Verfahrensweise muss noch auomatisiert und als Standard ins System implementiert werden. Es werden zwar auch ohne Ausrichtung von anisotropen Proben Messwerte generiert, diese sind jedoch aufgrund der Kreuzpolarisation nicht zur Auswertung geeignet.

Zusammengefasst ist die bildgebende Ellipsometrie eine der Messmethoden im Bereich der Oberflächenanalytik, die neben komplementären Techniken wie RAMAN oder AFM zusätzliche Informationen (optische Parameter, Schichtdicke) über die Probe liefert. 


\section{Probenpräparation}

Einige Proben wurden in Kooperation mit anderen Universitäten gefertigt. Alle Informationen über die Fertigung sind in diesem Unterkapitel zusammengetragen.

\subsection{Selbst hergestellte Proben}

\subsubsection{Hydrophilisierung}

Die Oberflächen von $\mathrm{SiO}_{2}$ auf Silizium-Substraten wurden vor weiteren Behandlungen wie Spin-Coating oder Microcontakt Printing zunächst gereinigt und anschließend hydrophilisiert. Dazu wurden die Substrate zunächst für $10 \mathrm{~min}$ in Ethanol (reinst.) gelegt und anschließend mit Reinstwasser gespült. Zur Hydrophilisierung wurden die Substrate für 30 min in eine Lösung aus NH3/H2O2/Reinstwasser (v/v, 1:1:5) bei $70^{\circ} \mathrm{C}$ gegeben. Nach Spülen mit Reinstwasser (3 mal) wurden die Substrate trocken gepustet und entweder sofort weiter behandelt oder in Reinstwasser gelagert.

\subsubsection{Spin-Coating}

Die Beschichtung der Substrate wurde nach Aktivierung der Oberfläche mittels Spin-Coating abgeschieden. Dazu wurden die jeweiligen Proben mit einer bestimmten Rotationsgeschwindigkeit gedreht und eine Lösung des jeweiligen Materials im geeigneten Lösungsmittel auf die Substrate gegeben. Nach Verflüchtigung des Lösungsmittels erhält man einen dünnen Film, dessen Schichtdicke von der Rotationsgeschwindigkeit sowie der Konzentration der Lösung abhängt. Es wurde der Spin Coater WS-650-23NPP der Firma Laurell verwendet.

\subsubsection{Mikrokontakt Printing}

Zur Funktionalisierung von $\mathrm{SiO}_{2}$-Oberflächen wurden PDMS-Stempel (Polydimethoxysilan, Sylgrad 184) verwendet. Diese wurden durch Aushärten der beiden Komponenten $(\mathrm{A}+\mathrm{B}, 10: 1)$ in einer mikrostrukturierten Form (40x40 $\mu \mathrm{m}$ Quadrate, $\mathrm{h}=10 \mu \mathrm{m}$, Abstand zwischen der Struktur $=20 \mu \mathrm{m})$ gefertigt. Die PDMS-Stempel wurden mit der Oberfläche in eine Lösung von Oktadekyltrimethoxysilan in Toluol (getrocknet, $\mathrm{c}=5 \mathrm{mM}$ und $1 \mathrm{mM}$ ) getaucht, um so den Stempel zu benetzen. Nachdem das Lösungsmittel sichtbar verdampft war, wurde der Stempel leicht auf die hydrophiliiserten Substrate gedrückt und dort für 30 min bei Raumtemperatur unter Druck 
gehalten. Nachdem der PDMS-Stempel von der Oberfläche genommen wurde, folgte die Reiniung der Proben mit Toluol, Reinstwasser und Isopropanol. Nach anschließender Trockung erfolgte die Vermessung der Proben. Die Stempel wurden für 5 min in Toluol gelagert um das restliche Silan von Oberfläche zu lösen. Die gesäuberten Stempel fanden anschließend weitere Verwendung.

\subsection{Kooperation}

\subsubsection{Laserablation}

Die Probe mit homogener PMMA-Schicht auf $\mathrm{SiO}_{2} / \mathrm{Si}(100)$ wurde vom Laserlaboratorium Göttingen mit einem gepulsten Argon-Ionen Laser $(\lambda=193 \mathrm{~nm})$, einer Fluenz im Bereich 100 - $500 \mathrm{~mJ} / \mathrm{cm}^{2}$ und 1 - 5 Pulsen unter Standardbedingungen an Luft bestrahlt. Das Linienschema hat eine Breite von ca. $30 \mu \mathrm{m}$ und der Abstand zwischen den Linien beträgt in etwa $1 \mathrm{~mm}$. Die Proben wurden von $\mathrm{T}$. Fricke-Begemann (LaserLaboratorium Göttingen e.V.) hergestellt und für ellipsometrische Untersuchungen überlassen.

\subsubsection{Mikroprägelithographie von PET}

Der mittels Mikroprägelithographie strukturierte amorphe PET-Film auf SiliziumSubstrat wurde von V. Bliznyuk (Clemson University) präpariert.

\subsection{3 $\mathrm{As}_{2} \mathrm{~S}_{3}$}

Auf einem Glas Substrat wurde eine Schicht an $\mathrm{As}_{2} \mathrm{~S}_{3}$ (amorph) mit einer Wachstumsrate von $10 \mathrm{~nm} / \mathrm{s}$ bei Raumtemperatur mittels thermischer Verdampfung im Vakuum ( $\mathrm{p}=10^{-5}$ Torr) abgeschieden. Anschließend wurde ein holographisches Gitter $(\mathrm{d}=20 \mu \mathrm{m})$ durch intereferometrische Holographie mit einem DPSS-Laser (diode pumped solid state, $\lambda=532 \mathrm{~nm}$ ) auf die Probe projeziert. Dabei wurde dieser Vorgang an 3 Stellen der gleichen Probe durchgeführt und die Belichtungszeit des Vorgangs variiert. Alle drei Belichtungszustände (unterbelichtet, korrekt belichtet und überbelichtet) wurden ellipsometrisch charakterisiert [47].

Die Proben wurden von A.Meshalkin (Academy of Sciences of Moldova) hergestellt und für ellipsometrische Studien zur Verfügung gestellt.

\subsubsection{Mikrokristalle}

Die anisotropen Mikrokristalle wuchsen durch Selbstorganisation unter Lösungsmittels Einfluss der Atmosphäre. Die Proben wurden von E. Y. Poimanova (Donetsk National University)

hergestellt. 


\subsubsection{PET-Folie}

Die PET-Folien wurden mit den Plastik Solar Zellen Materialien von F. Bammer (TU Wien) mittels Druckverfahren beschichtet und für ellipsometrischen Studien zur Verfügung gestellt.

\subsubsection{Graphen}

Die Graphenflocken wurden mittels Scotch-Tape-Methode von A. Matkovic (University of Belgrade) auf das Silizium-Substrat gebracht und für ellipsometrische Studien überlassen.

\subsubsection{Plastik Solar Zellen}

Die Synthese und Probenpräparation mittels Spin-Coating wurden für DPIDT-2T vom Arbeitskreis J. W. Rumer (Imperial College London) und die anderen Materialien (N2200, PTB-7, PCE 10, CPF4) von C. MCNeill (Monash University) durchgeführt und für ellipsometrische Untersuchungen freigegeben. 


\section{Appendix}




\section{Abbildungsverzeichnis}

2.1 lineare Polarisation . . . . . . . . . . . . . . . . . . . . . . . 12

2.2 zirkuläre Polarisation . . . . . . . . . . . . . . . . . . . . . 12

2.3 elliptische Polarisation . . . . . . . . . . . . . . . . . . . . . . . 13

2.4 Reflexion an einer Grenzfläche . . . . . . . . . . . . . . . . . . . . . 14

2.5 Brewsterwinkel SF10 . . . . . . . . . . . . . . . . . . . 16

2.6 Vergleich Pseudo-Brewsterwinkel . . . . . . . . . . . . . . . . . . 17

2.7 Totalreflektion . . . . . . . . . . . . . . . . . . . . . . 18

2.8 optische Interferenz . . . . . . . . . . . . . . . . . . . . . . . . . . . . 19

2.9 Dispersion von Weißlicht an einem Glasprisma. . . . . . . . . . . . 25

2.10 Dispersionsschema . . . . . . . . . . . . . . . . . . 26

2.11 Lorentz-Oszillator . . . . . . . . . . . . . . . . . . . . . . . . . . . . . . . . . . . . . . . . . . . .

2.12 Lorentz-Dispersion . . . . . . . . . . . . . . . . . . . 29

2.13 Vergleich Isolator, Halbleiter, Dye, Metall . . . . . . . . . . . . . . . . 29

2.14 Dispersion nach Sellmeier . . . . . . . . . . . . . . . . . 31

2.15 Dispersion Cauchy . . . . . . . . . . . . . . . . . . . 32

2.16 Urbach . . . . . . . . . . . . . . . . . . . . . . . . . . . . . . . . . . . . .

2.17 Gauss-Oszillator . . . . . . . . . . . . . . . . . . . . . . . . . . . . . . . 35

2.18 Drude-Modell . . . . . . . . . . . . . . . . . . 36

2.19 Tauc-Lorentz . . . . . . . . . . . . . . . . . . . . . . 38

2.20 Tauc-Lorentz-Urbach . . . . . . . . . . . . . . . . . . . . . . . . . . . . . . . . . . . . . . . . .

2.21 Sphärische Modelle . . . . . . . . . . . . . . . . . . . . . . . . . . . . . 40

2.22 Laminare Mosaikstruktur . . . . . . . . . . . . . . . . . . . 41

3.1 Delta-Psi vs. Schichtdicke von $\mathrm{SiO}_{2}(\lambda=658 \mathrm{~nm})$ als transparente Schicht auf Si (100). . . . . . . . . . . . . . . . . . . 43

3.2 Delta-Psi vs. Schichtdicke von $\mathrm{SiO}_{\mathrm{x}}(\lambda=658 \mathrm{~nm})$ als semi-transparente Schicht auf Si (100). . . . . . . . . . . . . . . . 43

3.3 Hochaufgelöstes Winkelspektrum eines transparenten Polymerfilms ( $\mathrm{d}=14042 \mathrm{~nm}, \mathrm{n}=1.4136)$ auf Si (100) bei $\lambda=658 \mathrm{~nm} . \quad . . . . \quad$. 44

3.4 Spektroskopische Messung an einem intransparenten Gold-Film und deren Dispersion im Vergleich zur Datenbank. . . . . . . . . . . . 45

3.5 Absorption und Desorption von Protein (bovine serum albumin, BSA) an hydrophilisierter $\mathrm{SiO}_{2}$ Oberfläche mit den ellipsometrischen Messdaten (links) und der berechneten Proteinschichtdicke (rechts). . . . . 46

3.6 Schematische Abbildung eines Nulling Ellipsometers in PCSA-Kinfiguration inklsuive Darstellung des Polarisationszustandes des Lichtstrahls. . . 47

3.7 Anzahl von Veröffentlichungen im Zeitraum von 1990-2015. . . . . . . 49

3.8 Aufbau einer EP4-SE. . . . . . . . . . . . . . . . . . . . . . . 50 
3.9 Region of Interest in einem gescannten Image einer mikrostrukturiten

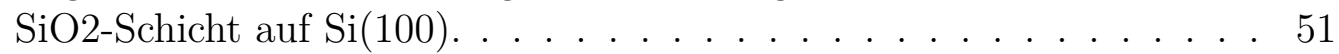

3.10 Bildkorrektur eines Grabs einer strukturierten $\mathrm{SiO} 2$ Schicht auf $\mathrm{Si}(100)$ aufgenommen bei einem AOI von $50^{\circ}$ und Verwendung des 50x Objektivs. . . . . . . . . . . . . . . . 52

3.11 Scanning Prozess . . . . . . . . . . . . . . . . 53

3.12 Mapping von Delta einer mikrostruktuirerten SiO2 Schicht auf $\mathrm{Si}(100) 54$

3.13 Schematischer Verlauf der Datenanalyse bei der Ellipsometrie. . . . . 56

3.14 FTIR-Spektrometer . . . . . . . . . . . . . . . . 58

3.15 Evaneszentes Feld . . . . . . . . . . . . . . . . . . 59

$3.16 \mathrm{AFM} \ldots \ldots \ldots \ldots \ldots$

3.17 Bestimmung des Kontaktwinkels von Reinstwasser an Al2O3 Oberfläche mittels dropshape_analysis (ImageJ) . . . . . . . . . 63

4.1 Delta map OTS SAM . . . . . . . . . . . . . . . . . . 65

4.2 3D Plot d-map OTS SAM . . . . . . . . . . . . . 66

4.3 3D-Plot der erhaltenen Schichtdickenkarte des mikrostruktuierten PMMAFilms (100 mJ, 5 Pulse) auf $\mathrm{SiO}_{2} / \mathrm{Si}$ (100) und Histogramm inklusive des Abtrags an PMMA durch Laserablation. . . . . . . . . . . . 67

4.4 Darstellung Abtrag von PMMA . . . . . . . . . . . . . . . 68

4.5 3D Darstellung der Schichtdickenkarten von mikrostrukturierten PETFilmen auf Siliziumsubstraten mit hexagonaler (links) und linearer (rechts) Anordnung. . . . . . . . . . . . . . . . . 69

4.6 3D Plot der AFM-Aufnahmen an mikrostruktirierten PET-Film auf Siliziumsubstraten mit hexagonaler und linearer Anordnung. . . . . . 70

4.7 Fotografie der Einkristalle des Thiophene-Phenylen Co-Oligomer auf Siliziumsubstrate mit thermischen $\mathrm{SiO}_{2}(\mathrm{~d}=300 \mathrm{~nm}) . \quad \ldots . . . \quad .75$

4.8 Ellipsometrische Kontrastkarte der Einkristalle bei AOI $=60^{\circ}$ und $\lambda=510 \mathrm{~nm}$ inklusive Markierung der Messbereiche der spektroskopischen Untersuchungen an Multilayer (gelb) und Monolayer (blau). . . 76

4.9 Links: Ellipsometrische Kontrastkarte des Einkristalls mit Multilagen $\left(\mathrm{AOI}=60^{\circ}, \lambda=600 \mathrm{~nm}\right.$ und $\Theta=55^{\circ}$ ), rechts: graphische Darstellung des detektierten Signals bei p- bzw. s-polarisierten Licht zur Bestimmung des pseudo-isotropen Punktes. . . . . . . . . . . . . . 77

4.10 Graphische Darstellung von Delta/Psi-Wellenlängenspektren $(\lambda=400$ $750 \mathrm{~nm}, 5 \mathrm{~nm}$ Intervalle) bei zwei verschieden Einfallswinkeln $\left(\mathrm{AOI}=50^{\circ}\right.$, $\left.60^{\circ}\right)$ an den pseudo-isotropen Punkten $\left(\Theta=55^{\circ}, 145^{\circ}\right)$ von HexTTBTT-Hex. . . . . . . . . . . . . . . 77

4.11 Dispersion von $\mathrm{n}$ (Linie) und $\mathrm{k}$ (Punkte) des biaxial anisotropen organischen Halbleitermaterials Hex-TTBTT-Hex $(\mathrm{d}=41.2 \pm 0.2 \mathrm{~nm}) . \quad 78$

4.12 Schichtdickenkarte des mehrschichtigen Einkristalls $\left(\Theta=145^{\circ}\right)$, sowie die quantiative Analyse zur Bestimmung der molekularen Schicht $\left(\mathrm{d}_{\text {molekulare Schicht }}=3.49 \pm 0.2 \mathrm{~nm}\right)$. 
4.13 Links: Ellipsometrische Kontrastkarte des Einkristalls mit Multilagen (AOI $=60^{\circ}, \lambda=600 \mathrm{~nm}$ und $\Theta=12^{\circ}$ ), rechts: graphische Darstellung des detektierten Signals bei p- bzw. s-polarisierten Licht zur Bestimmung des pseudo-isotropen Punktes. . . . . . . . . . . . .

4.14 Graphische Darstellung von Delta/Psi-Wellenlängenspektren $(\lambda=400$ $750 \mathrm{~nm}, 5 \mathrm{~nm}$ Intervalle) bei zwei verschieden Einfallswinkeln $\left(\mathrm{AOI}=50^{\circ}, 60^{\circ}\right)$ an den pseudo-isotropen Punkten $\left(\Theta=12^{\circ}, 102^{\circ}\right)$ von Monolagen Hex-TTBTT-Hex $\left(\mathrm{d}_{\text {Monolage }}=3.09 \pm 0.07 \mathrm{~nm}\right) . \ldots . . . . . .881$

4.15 Delta- und Psi-Mittelwerte und deren Standardabweichung für die Messungen des Standardsysteme vom $\mathrm{SiO}_{2}$ (30.6 nm (links), $116.8 \mathrm{~nm}$ (rechts)) auf Si (100) in Abhängigkeit vom Einfallswinkel. . . . . . . .

4.16 Gradient in Y-Richtung der ellipsometrischen Maps in Abhängigkeit vom Einfallswinkel für $\mathrm{SiO}_{2}$ (30.6 nm (links), $116.8 \mathrm{~nm}$ (rechts)) auf

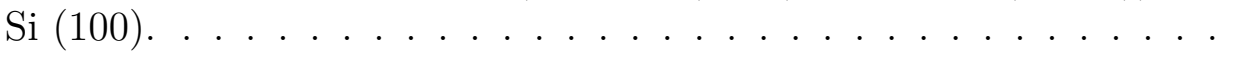

4.17 Sensitivität (oben) und relativierter über die Objektive gemittelter Gradient in Y-Richtung (unten) der beiden Proben bei unterschiedlicher Schichtdicke für $\mathrm{SiO}_{2}$ auf $\mathrm{Si}$ (100) in Abhängigkeit vom Einfallswinkel. . . . . . . . . . . . . . . . . . . 84

4.18 Kalibrierung der Luft-Wasser-Grenzfläche UltraBAM. . . . . . . . . . 85

4.19 LB-Isotherme, UltraBAM Bilder sowie Histogramme der berechneten Schichtdicke von 4-Cyano-4-[(dodecylsulfanylthiocarbonyl)sulfanyl]pentansäure. 86

4.20 Delta-/Schichtdicken-Map (AOI $=70^{\circ}, \lambda=658 \mathrm{~nm}$ ) und Histogramme des LB-Film Übertrags vom RAFT-Agenz auf $\mathrm{SiO}_{2} / \mathrm{Si}(100)$. . . 87

4.21 Prinzip des Beamcutters bei der abbildenden Ellipsometrie inklusive Strahlengang . . . . . . . . . . . . . . . . . 88

4.22 Beamcutter der Ep3 Generation (links) mit Befestigung am Laserarm und rechts am z-Lift senkrecht zur Probenebene (Ep4) [1] . . . . . . 89

4.23 Theta Scan PET-Film (amorph) und PET-Folie (kristallin). . . . . . 91

4.24 Brechungsindex der PET-Folie bei $658 \mathrm{~nm}$ in Abhängigkeit von Theta. 92

4.25 Delta/Psi-Wellenlängenspektrum ITO auf PET Folie. . . . . . . . . . 94

4.26 Dispersion ITO auf PET-Folie. . . . . . . . . . . . . . . . . . . 94

4.27 Delta/Psi-Wellenlängenspektrum PEDOT:PSS auf PET Folie. . . . . 95

4.28 Erhaltene Dispersion von PEDOT:PSS auf PET-Folie. . . . . . . . . 96

4.29 Delta-/Psi-Wellenlängenspektrum von P3HT:PCBM auf PET Folie. . 97

4.30 Ermittelte Dispersion von P3HT:PCBM auf PET-Folie. . . . . . . . . 98

4.31 Delta-Map und Säulendiagramm der Schichtdicken von P3HT:PCBM des ROI-Profils. . . . . . . . . . . . . . . . . 98

4.32 Übersicht über die Schichtdickenvariation von P3HT:PCBM auf PETFolie innerhalb der ROI-Profile in Abhängikeit von der Liniendichte.

4.33 Übersicht der gemittelten Schichtdicke und des relativen Fehlers der P3HT:PCBM-Schicht auf PET-Folie in Abhängigkeit der Liniendichte. 100 4.34 Delta-/Psi-Wellenlängenspektrum $\mathrm{AlQ}_{3}$ auf PET Folie. . . . . . . . . 101

4.35 Dispersion von $\mathrm{AlQ}_{3}$ auf PET. . . . . . . . . . . . . . . . . . . . . . . 102

4.36 Delta-/Psi-Wellenlängenspektren von PEDOT:PSS auf Glas bei variiender Rotationsgeschwindigkeit. . . . . . . . . . . . . . . . 103 
4.37 Schichtdicke versus Rotationsgeschwindigkeit PEDOT:PSS. . . . . . . 104

4.38 ATR-FTIR Spektrum von PEDOT:PSS auf Glas. . . . . . . . . . . . 104

4.39 (Links) Fingerprint Bereich des ATR-FTIR-Spektrums von PEDOT:PSS

Filmen bei variierender Roataionsgeschwindigkeit des Herstellunsgprozesses sowie (rechts) lineare Korrelation zwischen Schichtdicke und Fläche der Absorptionsbande. . . . . . . . . . . . . . . . . . . 105

4.40 Delta-/Psi-Wellenlängenspektren von P3HT:PCBM auf Glas bei variierender Rotationsgeschwindigkeit. . . . . . . . . . . . . 106

4.41 Schichtdicke versus Rotationsgeschwindigkeit von P3HT:PCBM. . . . 106

4.42 ATR-FTIR Spektrum von P3HT:PCBM auf Glass. . . . . . . . . . . 107

4.43 (Links) Fingerprint Bereich des ATR-FTIR-Spektrums von P3HT:PCBM Filmen mit variierender Roataionsgeschwindigkeit beim Herstellunsgprozess sowie (rechts) lineare Korrelation zwischen Schichtdicke und Fläche der CH-Absorptionsbande. . . . . . . . . . . . . . . 107

4.44 Spektroskopische Ellipsometrie von $\mathrm{SiO}_{2}$ auf $\mathrm{Si}$ (100) unter Verwendung verschiedener Lichtquellen (oben: Filterrad $\left(\Delta \lambda_{\mathrm{sp}}=10 \mathrm{~nm}\right)$, mitte: Monochromator $\left(\Delta \lambda_{\mathrm{sp}}=5 \mathrm{~nm}\right)$, unten: durchstimmbarer Laser $\left(\Delta \lambda_{\mathrm{sp}}=1.8 \mathrm{~nm}\right) . \ldots \ldots \ldots . \ldots \ldots 11$

4.45 Bandbreiten Effekt bei der Simulation von Delta/Psi-Wellenlängenspektren

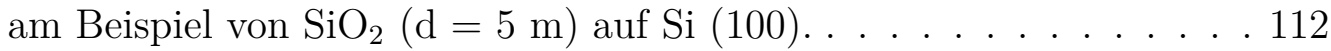

4.46 Delta/Psi-Wellenlängenspektrum von homogenen $\mathrm{As}_{2} \mathrm{~S}_{3}$ Film $(\mathrm{d}=960.5 \mathrm{~nm}$ $\pm 0.1 \mathrm{~nm})$ auf Glas. . . . . . . . . . . . . . . . . . . 113

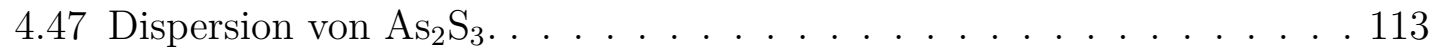

4.48 Delta/Psi-Wellenlängenspektrum $\mathrm{SiN}_{3}$ auf $\mathrm{Si}(100)$. . . . . . . . . . . 114

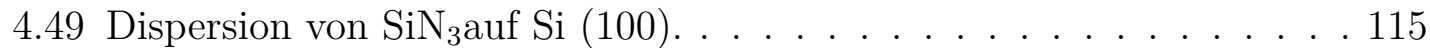

4.50 Vergleich der Delta/Psi-Wellenlängenspektren von DPIDT-T2 (500

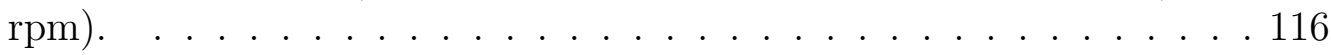

4.51 Dispersion DPIDT-T2 (as cast, annealed) . . . . . . . . . . . . 117

4.52 Gemessene und simultierte Delta/Psi-Wellenlängenspektren von N2200 (Xylene) auf Si (100) . . . . . . . . . . . . . . . . . . . 118

4.53 Dispersion von N2200 (Xylene) auf Si (100) der ROI-basierten Messungen. . . . . . . . . . . . . . . . . . . . . . 119

4.54 Ellipsometrisches Kontrastbild (10x Objektiv, $\lambda=440 \mathrm{~nm}$ ) und ROI Profil N2200 (Xylene) auf Si (100). . . . . . . . . . . . . . . . 120

4.55 Delta/Psi-Werte in Abhängikeit von der Wellelänge und der dazugehörigen Dispersion des N2200-Films $(\mathrm{d}=125.6 \pm 10.7 \mathrm{~nm}$, Chlorbenzol) auf Si (100). . . . . . . . . . . . . . . . . . . . 121

4.56 Delta/Psi-Werte in Abhängikeit von der Wellelänge und der dazugehörigen Dispersion PTB7-Films $(\mathrm{d}=44.7 \pm 1.2 \mathrm{~nm})$ auf Si (100)

4.57 Delta/Psi-Werte in Abhängikeit von der Wellenlänge und der dazugehörigen Dispersion des PCE10-Films ( $\mathrm{d}=84.2 \pm 1.2 \mathrm{~nm})$ auf Si (100)

4.58 Delta/Psi-Werte in Abhängikeit von der Wellelänge und der dazugehörigen Dispersion CPF-Films $(\mathrm{d}=67.6 \pm 1.1 \mathrm{~nm})$ auf Si (100). . . . 123 
4.59 Delta/Psi-Werte in Abhängikeit von der Wellelänge und der dazugehörigen Dispersion PTB7:N2200-Films (1:1.2, $\mathrm{d}=85.6 \pm 2.9 \mathrm{~nm})$ auf

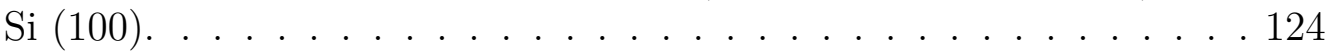

4.60 Delta/Psi-Werte in Abhängikeit von der Wellelänge und der dazugehörigen Dispersion PCE10:N2200-Films (1:1, d = $120.4 \pm 3.8 \mathrm{~nm})$

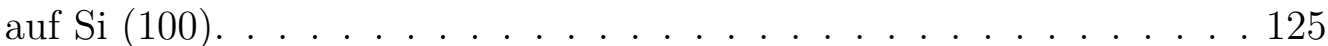

4.61 Delta/Psi-Werte in Abhängikeit von der Wellelänge und der dazugehörigen Dispersion CPF-4:N2200-Films $(2: 1, \mathrm{~d}=98.8 \pm 1.5 \mathrm{~nm})$ auf

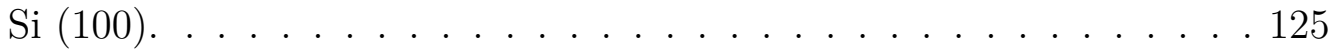

4.62 UV/NIR-Messung von Graphenflocken auf Si (100) mit $392.3 \mathrm{~nm} \mathrm{SiO}_{2}$ mit Delta-Map bei $480 \mathrm{~nm} . \quad$. . . . . . . . . . . . . . . . . . . 126

4.63 Vergleich der Dispersionen von Graphen modelliert mittels Fanoresonanz und Drude-Lorentz-Term. . . . . . . . . . . . . . . . . . . . 127

4.64 Übersicht der Messergebnisse eines Linienprofils spektroskopischer Maps von Graphen auf $\mathrm{Si}$ (100) mit $\mathrm{SiO}_{2}$ im UV/VIS/NIR-Bereich. . 129

4.65 Übersicht der Messergebnisse eines Linienprofils spektroskopischer Maps von $\mathrm{As}_{2} \mathrm{~S}_{3}$ auf Glas im UV/VIS Bereich.

4.66 Spektroskopische Messung von 360-1700 nm (4 Zonen) an intransparenter Goldoberfläche ohne Korrektur des Kompensators und Mittelwerte bzw. Abweichungen von Delta und Psi aus den 4 Zonen. . . . . 131

4.67 Retardierung und Achsenshift des Kompensators und spektrokopische 4 Zonen Messung mit Kompensatorkorrektur. . . . . . . . . . . . . . 132

4.68 Delta-Map einer Probe mit vier Messbereichen verschiedener $\mathrm{SiO}_{2}{ }^{-}$ Schichtdicken auf Si (100) bei $500 \mathrm{~nm}$. . . . . . . . . . . . . 133

4.69 Glasübergangstemperatur von Polystyrol $(\mathrm{d}=112 \mathrm{~nm}) . \quad \ldots . . . .137$

4.70 Zersetzungstemperatur von Polystyrol $(\mathrm{d}=54 \mathrm{~nm}) . \quad \ldots . . .138$

4.71 Light-guides bei Messung an der flüssig-flüssig-Grenzfläche [1] . . . . . 139

4.72 AOI-Spektrum an der Parafinöl/Wasser-Grenzfläche bei einer Wellenlänge von $658 \mathrm{~nm} . \quad$. . . . . . . . . . . . . . . . . . . . . 140

4.73 Delta/Psi-Wellenlängenspektrum an der Parafinöl/Wasser-Grenzfläche bei einem Einfallswinkel von $40^{\circ}$. . . . . . . . . . . . . . . . . . . 141

4.74 Delta/Psi-Wellenlängenspektrum eines anisotropen Polystyrolfilms (Dispersion, rechts) an der Parafinöl/Wasser-Grenzfläche bei einem Einfallswinkel von 40․ . . . . . . . . . . . . . . . . . . . . 141

4.75 Psi- und Schichtdickenkarte eines PS-Films an der Öl/Wasser-Grenzfläche bei $658 \mathrm{~nm}$. . . . . . . . . . . . . . . . . . . . . . 142

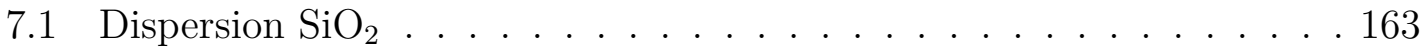

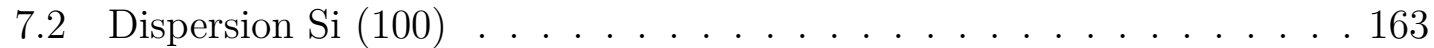

7.3 Dispersion PMMA . . . . . . . . . . . . . . . . . . . . . . . . . . 164

7.4 Dispersion PS . . . . . . . . . . . . . . . . . . . . . . . 164

7.5 Dispersion PET (amorph) . . . . . . . . . . . . . 165 


\section{Tabellenverzeichnis}

2.1 Jones-Vektoren für Polarisationszustände . . . . . . . . . . . . . . . . 13

2.2 Beziehungen optische Konstanten . . . . . . . . . . . . . . . . . . 24

3.1 Laterale Aufösung und Größe des Sichtfgeldes in x-Richtung der eingesetzten Objektive. . . . . . . . . . . . . . . . 51

4.1 Übersicht Schichtdicke von $\mathrm{SiO}_{2}$ und mikropatterned OTS. . . . . . . 66

4.2 Abtrag PMMA . . . . . . . . . . . . . . . . . 68

4.5 Schichtdicke und optische Parameter $(\lambda=658 \mathrm{~nm})$ von $\mathrm{As}_{2} \mathrm{~S}_{3}$ bei verschiedenen Belichtungszeiten. . . . . . . . . . . . . . 72

4.3 3D-Darstellung zugeschnittener Delta/Psi-Maps und Histogramme der gesamten Delta/Psi-Maps $(\lambda=658 \mathrm{~nm})$ von $\mathrm{As}_{2} \mathrm{~S}_{3}$ bei variierender Belichtung. . . . . . . . . . . . . . . . . 73

4.4 3D-Darstellung zugeschnittener $\mathrm{n} / \mathrm{k}$-Maps und Histogramme der gesamten $\mathrm{n} / \mathrm{k}$-Maps $(\lambda=658 \mathrm{~nm})$ von $\mathrm{As}_{2} \mathrm{~S}_{3}$ bei variierender Belichtung. 74

4.6 Delta-Maps (links, AOI $=50^{\circ}, \lambda=550 \mathrm{~nm}$ ) der Monolagen des Einkristalls an den pseudo-istropen Punkten $\left(\Theta=12^{\circ}, 102^{\circ}\right)$ und die resultierenden Schichtdickenkarten (mitte) inklusive Histogramm Darstellung (rechts) zur quantitativen Bestimmung der Schichtdicke der Monolage $\left(\mathrm{d}_{\text {Monolage }}=3.16 \pm 0.09 \mathrm{~nm}\right) \ldots \ldots$. . . . . . . 80

4.7 Brechungsindex von uniaxial anisotroper PET-Folie. . . . . . . . . . . 92

4.8 Kontaktwinkels von Wasser auf verschiedenen Materialien. . . . . . . 109

4.9 Tauc-Lorentz Parameter von $\mathrm{As}_{2} \mathrm{~S}_{3}$. . . . . . . . . . . . . . . . . . . . 114

4.10 Tauc-Lorentz Parameter von $\mathrm{SiN}_{3}$. . . . . . . . . . . . . . . . . . . 115

4.11 Schichtdicke von DPIDT-2T Filmen bei variierender Rotationsgeschwindigkeit beim Herstellungsprozess. . . . . . . . . . . . . . . . . . 117

4.12 Modelparameter für DPIDT-2T Filme. . . . . . . . . . . . . . . . . 118

4.13 Modelparameter für PCE10. . . . . . . . . . . . . . . . . . . . 123

4.14 Einfluss der Auslenkung (ranges) der optischen Komponenten auf die Bestimmung der Schichtdicke bei spektroskopischen Messungen. . . . 133

4.15 Fit-Ergebnisse der $\mathrm{SiO}_{2}$-Probe unter Verwendung des traditionellen Nullings. . . . . . . . . . . . . . . . . . . . . . . . . . . . . . . . 134

4.16 Fit-Ergebnisse der $\mathrm{SiO}_{2}$-Probe unter Verwendung des Ep4-Nullings. . 134 


\section{Literaturverzeichnis}

[1] Broschüre ep4. Technical report, Accurion GmbH, 2016.

[2] Ole Albrektsen, René Lynge Eriksen, Sergey M Novikov, D Schall, M Karl, Sergey I Bozhevolnyi, and Adam Cohen Simonsen. High resolution imaging of few-layer graphene. Journal of Applied Physics, 111(6):064305, 2012.

[3] MI Alonso, S Funke, A González, M Garriga, PO Vaccaro, AR Goñi, A Ruiz, $\mathrm{M}$ Alonso, and $\mathrm{PH}$ Thiesen. Spectroscopic imaging ellipsometry of selfassembled sige/si nanostructures. Applied Surface Science, 2016.

[4] Mirjam Andreasson-Ochsner, Zhikang Fu, Sylvia May, Low Ying Xiu, Madhavan Nallani, and Eva-Kathrin Sinner. Selective deposition and self-assembly of triblock copolymers into matrix arrays for membrane protein production. Langmuir, 28(4):2044-2048, 2012.

[5] Hans Arwin, Stefan Welin-Klintström, and Roger Jansson. Off-null ellipsometry revisited: basic considerations for measuring surface concentrations at solid/liquid interfaces. Journal of colloid and interface science, 156(2):377-382, 1993.

[6] David E Aspnes. Optical properties of thin films. Thin solid films, 89(3):249$262,1982$.

[7] Rasheed MA Azzam and Nicholas Mitchell Bashara. Ellipsometry and polarized light. North-Holland. sole distributors for the USA and Canada, Elsevier Science Publishing Co., Inc., 1987.

[8] Indu Bala and Santanu Kumar Pal. Rod-disc oligomeric liquid crystal based on 4-cyanobiphenyl and truxene core. Liquid Crystals, 43(7):963-971, 2016.

[9] D Beaglehole. Performance of a microscopic imaging ellipsometer. Review of scientific instruments, 59(12):2557-2559, 1988.

[10] Dwight W Berreman. Optics in stratified and anisotropic media: $4 \times 4$-matrix formulation. JOSA, 62(4):502-510, 1972.

[11] Gianangelo Bracco and Bodil Holst. Surface science techniques. Springer Science \& Business Media, 2013.

[12] Madhumita Choudhuri, Alokmay Datta, Chitra Murli, D Bhattacharyya, and SC Gadkari. Long-timescale dynamics of thiol capped au nanoparticle clusters at the air-water interface. In AIP Conference Proceedings, volume 1591, pages 904-906. AIP, 2014.

[13] GB DeMaggio, WE Frieze, DW Gidley, Ming Zhu, HA Hristov, and AF Yee. Interface and surface effects on the glass transition in thin polystyrene films. Physical Review Letters, 78(8):1524, 1997. 
[14] Wolfgang Demtröder. Experimentalphysik 2. Elektrizität und Optik, Kapitel, $11,1995$.

[15] AB Djurišić, CY Kwong, TW Lau, EH Li, ZT Liu, HS Kwok, LSM Lam, and WK Chan. Optical functions of tris (8-hydroxyquinoline) aluminum (alq3) by spectroscopic ellipsometry. Applied Physics A, 76(2):219-223, 2003.

[16] Paul Drude. Ueber die gesetze der reflexion und brechung des lichtes an der grenze absorbirender krystalle. Annalen der Physik, 268(12):584-625, 1887.

[17] Mart-Mari Duvenhage, Martin Ntwaeaborwa, Hendrik G Visser, Pieter J Swarts, Jannie C Swarts, and Hendrik C Swart. Determination of the optical band gap of alq 3 and its derivatives for the use in two-layer oleds. Optical Materials, 42:193-198, 2015.

[18] Editorial. It's still all about graphene. Nature Materials, 10:1, 2011.

[19] Hans Elwing. Protein absorption and ellipsometry in biomaterial research. Biomaterials, 19(4-5):397-406, 1998.

[20] Simon Faiss, Steffen Schuy, Daniela Weiskopf, Claudia Steinem, and Andreas Janshoff. Phase transition of individually addressable microstructured membranes visualized by imaging ellipsometry. The Journal of Physical Chemistry $B, 111(50): 13979-13986,2007$.

[21] AS Ferlauto, GM Ferreira, JM Pearce, CR Wronski, RW Collins, Xunming Deng, and Gautam Ganguly. Analytical model for the optical functions of amorphous semiconductors from the near-infrared to ultraviolet: Applications in thin film photovoltaics. Journal of Applied Physics, 92(5):2424-2436, 2002.

[22] Hiroyuki Fujiwara. Spectroscopic ellipsometry: principles and applications. John Wiley \& Sons, 2007.

[23] S Funke, B Miller, E Parzinger, P Thiesen, AW Holleitner, and U Wurstbauer. Imaging spectroscopic ellipsometry of mono-and few-layer mos2. arXiv preprint arXiv:1603.06644, 2016.

[24] Henri Gavin. The levenberg-marquardt method for nonlinear least squares curve-fitting problems, 2011.

[25] Andre K Geim and Konstantin S Novoselov. The rise of graphene. Nature materials, 2007.

[26] HU Gremlich and H Günzler. Ir-spektroskopie: Eine einführung, 2003.

[27] DJ Hayton and TE Jenkins. On the frustration of back-surface reflection from transparent substrates in ellipsometry. Measurement Science and Technology, 15(2):N17, 2003.

[28] James N Hilfiker, Brandon Pietz, Bill Dodge, Jianing Sun, Nina Hong, and Stefan Schoeche. Spectroscopic ellipsometry characterization of coatings on biaxially anisotropic polymeric substrates. Applied Surface Science, 2016.

[29] Andrew R Hind, Suresh K Bhargava, and Anthony McKinnon. At the solid/liquid interface: Ftir/atr - the tool of choice. Advances in colloid and interface science, 93(1):91-114, 2001. 
[30] Dirk Hoenig. Charakterisierung von Monofilmen and Wasser/Luft-Grenzfläche durch Brewster-Winkel-Mikroskopie. PhD thesis, 1991.

[31] GE Jellison Jr and FA Modine. Parameterization of the optical functions of amorphous materials in the interband region. Applied Physics Letters, 69(3):371-373, 1996.

[32] Gang Jin, Roger Jansson, and Hans Arwin. Imaging ellipsometry revisited: developments for visualization of thin transparent layers on silicon substrates. Review of scientific instruments, 67(8):2930-2936, 1996.

[33] Peter B Johnson and R-W_ Christy. Optical constants of the noble metals. Physical review B, 6(12):4370, 1972.

[34] Stefka Nikolova Kasarova, Nina Georgieva Sultanova, Christo Dimitrov Ivanov, and Ivan Dechev Nikolov. Analysis of the dispersion of optical plastic materials. Optical Materials, 29(11):1481-1490, 2007.

[35] Yusuke Kobayashi, Rina Hirai, Daiki Ito, Yasushi Yamamoto, Keijiro Taga, and Zameer Shervani. Morphology observation of novel 3, 4-bis-alkyloxycarbonylhexanedioic acid monolayer on water surface by dropping method. Journal of Biophysical Chemistry, 7(02):43, 2016.

[36] Erik Langereis, SBS Heil, HCM Knoops, W Keuning, MCM Van De Sanden, and WMM Kessels. In situ spectroscopic ellipsometry as a versatile tool for studying atomic layer deposition. Journal of Physics D: Applied Physics, 42(7):073001, 2009.

[37] A Laskarakis and S Logothetidis. On the optical anisotropy of poly (ethylene terephthalate) and poly (ethylene naphthalate) polymeric films by spectroscopic ellipsometry from visible-far ultraviolet to infrared spectral regions. Journal of applied physics, 99(6):066101, 2006.

[38] Yongye Liang, Zheng Xu, Jiangbin Xia, Szu-Ting Tsai, Yue Wu, and Gang Li. For the bright future bulk heterojunction polymer solar cells with power conversion efficiency of 7.4\%. Advanced Materials, 22(20), 2010.

[39] Xueliang Liu, Sven Huettner, Zhuxia Rong, Michael Sommer, and Richard H Friend. Solvent additive control of morphology and crystallization in semiconducting polymer blends. Advanced Materials, 24(5):669-674, 2012.

[40] M Losurdo, M Giangregorio, P Capezzuto, G Bruno, R De Rosa, F Roca, C Summonte, J Pla, and R Rizzoli. Parametrization of optical properties of indium-tin-oxide thin films by spectroscopic ellipsometry: Substrate interfacial reactivity. Journal of Vacuum Science \&5 Technology A, 20(1):37-42, 2002.

[41] Obrift-Lieutenant Malus. Eine neue optische erscheinung, die polarisirung der lichtstrahlen betreffend. Annalen der Physik, 38(7):237-248, 1811.

[42] Aleksandar Matković, Angela Beltaos, Marijana Milićević, Uroš Ralević, Borislav Vasić, Djordje Jovanović, and Radoš Gajić. Spectroscopic imaging ellipsometry and fano resonance modeling of graphene. Journal of Applied Physics, 112(12):123523, 2012. 
[43] D De Sousa Meneses, M Malki, and P Echegut. Structure and lattice dynamics of binary lead silicate glasses investigated by infrared spectroscopy. Journal of non-crystalline solids, 352(8):769-776, 2006.

[44] Cheng Mu, Peng Liu, Wei Ma, Kui Jiang, Jingbo Zhao, Kai Zhang, Zhihua Chen, Zhanhua Wei, Ya Yi, Jiannong Wang, et al. High-efficiency all-polymer solar cells based on a pair of crystalline low-bandgap polymers. Advanced $M a$ terials, 26(42):7224-7230, 2014.

[45] Mette Marie Bruun Nielsen and Adam Cohen Simonsen. Imaging ellipsometry of spin-coated membranes: mapping of multilamellar films, hydrated membranes, and fluid domains. Langmuir, 29(5):1525-1532, 2013.

[46] AL Richard et al. Interface and surface effects on the glass-transition temperature in thin polymer films. Faraday Discussions, 98:219-230, 1994.

[47] C Roeling, P Thiesen, A Meshalkin, E Achimova, V Abashkin, A Prisacar, and $\mathrm{G}$ Triduh. Imaging ellipsometry mapping of photo-induced refractive index in as 2 s 3 films. Journal of Non-Crystalline Solids, 365:93-98, 2013.

[48] Joseph W Rumer, Sheng-Yao Dai, Matthew Levick, Youngju Kim, MarieBeatrice Madec, Raja S Ashraf, Zhenggang Huang, Stephan Rossbauer, Bob Schroeder, Laure Biniek, et al. Dihydropyrroloindoledione-based copolymers for organic electronics. Journal of Materials Chemistry C, 1(15):2711-2716, 2013 .

[49] Dirk Schmaljohann, Mirko Nitschke, Roland Schulze, Andreas Eing, Carsten Werner, and Klaus-Jochen Eichhorn. In situ study of the thermoresponsive behavior of micropatterned hydrogel films by imaging ellipsometry. Langmuir, 21(6):2317-2322, 2005.

[50] Steffen Schuy, Simon Faiss, Nicholas C Yoder, Venkateshwarlu Kalsani, Krishna Kumar, Andreas Janshoff, and Reiner Vogel. Structure and thermotropic phase behavior of fluorinated phospholipid bilayers: a combined attenuated total reflection ftir spectroscopy and imaging ellipsometry study. The Journal of Physical Chemistry B, 112(28):8250-8256, 2008.

[51] Aurélien F Stalder, Tobias Melchior, Michael Müller, Daniel Sage, Thierry Blu, and Michael Unser. Low-bond axisymmetric drop shape analysis for surface tension and contact angle measurements of sessile drops. Colloids and Surfaces A: Physicochemical and Engineering Aspects, 364(1):72-81, 2010.

[52] RA Synowicki. Suppression of backside reflections from transparent substrates. physica status solidi (c), 5(5):1085-1088, 2008.

[53] RA Synowicki and Thomas E Tiwald. Optical properties of bulk c-zro 2, c-mgo and a-as 2 s 3 determined by variable angle spectroscopic ellipsometry. Thin Solid Films, 455:248-255, 2004.

[54] Yaqi Tang and Christopher R McNeill. All-polymer solar cells utilizing low band gap polymers as donor and acceptor. Journal of Polymer Science Part B: Polymer Physics, 51(6):403-409, 2013. 
[55] Harland Tompkins and Eugene A Irene. Handbook of ellipsometry. William Andrew, 2005.

[56] Harland G Tompkins and William A McGahan. Spectroscopic ellipsometry and reflectometry: a user's guide. Wiley, 1999.

[57] A Tsanova, A Jordanova, T Dzimbova, T Pajpanova, E Golovinsky, and Z Lalchev. Interaction of methionine-enkephalins with raft-forming lipids: monolayers and bam experiments. Amino acids, 46(5):1159-1168, 2014.

[58] Yuliang Wang and Marya Lieberman. Growth of ultrasmooth octadecyltrichlorosilane self-assembled monolayers on sio2. Langmuir, 19(4):1159-1167, 2003.

[59] JW Weber, VE Calado, and MCM Van de Sanden. Optical constants of graphene measured by spectroscopic ellipsometry. Applied Physics Letters, 97(9):091904, 2010.

[60] James L Wilbur, Amit Kumar, Enoch Kim, James L Whitesides, George MWilbur, Amit Kumar, Enoch Kim, and George M Whitesides. Microfabrication by microcontact printing of self-assembled monolayers. Advanced Materials, 6(78):60-604, 1994.

[61] Joshua D Willott, Timothy J Murdoch, Ben A Humphreys, Steve Edmondson, Grant B Webber, and Erica J Wanless. Critical salt effects in the swelling behavior of a weak polybasic brush. Langmuir, 30(7):1827-1836, 2014.

[62] Ulrich Wurstbauer, Christian Röling, Ursula Wurstbauer, Werner Wegscheider, Matthias Vaupel, Peter H Thiesen, and Dieter Weiss. Imaging ellipsometry of graphene. arXiv preprint arXiv:1008.3206, 2010.

[63] EA Zakhidov, AM Kokhkharov, Sh K Nematov, RA Nusretov, N Sh Ashurov, VO Kuvondikov, AA Saparbaev, EP Normatov, and BA Normuminov. Spectral characteristics of a carotenoid-porphyrin-fullerene supramolecule, a promising material for organic photovoltaic devices. Applied Solar Energy, 51(3):195-201, 2015.

[64] Shaoqing Zhang, Long Ye, Wenchao Zhao, Delong Liu, Huifeng Yao, and Jianhui Hou. Side chain selection for designing highly efficient photovoltaic polymers with 2d-conjugated structure. Macromolecules, 47(14):4653-4659, 2014. 


\section{Abkürzungsverzeichnis}

$\alpha$ Absorptionskoeffizient

$\beta$ Pasenveränderung

$\Delta$ Phasenshift

$\delta$ Phase

$\epsilon$ dielektische Konstante

$\epsilon_{0}$ Dielektrizitätskonstante im Vakuum

$\Gamma$ Dämpfungskonstante

$\Gamma$ Dämpfungskonstante

$\gamma_{f}$ Dämpfungskonstante Drude Modell

$\in$ Element von

$\lambda_{p}$ Plasmawellenlänge Drude Modell

$\mathbb{C}$ komplexe Zahl

$\omega$ Kreisfrequenz

$\omega_{0}$ Resonanzfrequenz

$\omega_{p}$ Plasmafrequenz Drude Modell

$\phi_{B}$ Brewsterwinkel

$\phi_{i}$ Einfallswinkel

$\phi_{r}$ Reflexionswinkel

$\phi_{t}$ Brechwinkel

$\rho$ komplexes Verhältnis der Reflektionskoeffizient

$\sigma$ Leitfähigskonstante

$0 \quad$ Material 0 
1 Material 1

i einfallend

$p \quad$ parallel

$r$ reflektiert

$s$ senkrecht

$t$ transmittiert

A Amplitude

A Cauchy Parameter

$B$ Halbwertsbreite Gauss Modell

$B$ Sellmeier Parameter

$C$ Sellmeier Parameter

c Lichtgeschwindigkeitskonstante

d Schichtdicke

$E$ Elektrisches Feld

e Elektronenladung

$E_{0}$ Resonanzenergie

$E_{1}$ Offesetenergie Tauc-Lorentz-Urbach Modell

$E_{b}$ Bandlücke Urbach Modell

$E_{c}$ Resonanzerergie Gauss Modell

$E_{g}$ Bandlückel

$E_{p}$ Parameter Cody-Lorentz-Urbach Modell

$E_{u}$ Urbachenergie Tauc-Lorentz-Urbach Modell

$f_{a}$ Fraktionsfaktor

$G_{C}$ Codyterm Cody-Lorentz-Urbach Modell

$G_{T}$ Tauc Term Tauc-Lorentz Modell

$k$ Extinktionskoeffizient

$k_{z}$ Absorptionskoeffizient 
lambda Wellenlänge

$m_{e}$ Elektronenmasse

$n$ Brechungsindex

$q$ Screening faktor

$R$ Reflexion

$r$ Fresnelsche Reflexionskoeffizient

$t$ Fresnelsche Transmissionskoeffizient

$t$ Zeit

$U_{1}$ Urbachparameter Urbach Modell

$U_{2}$ Urbachenergie Urbach Modell

Ñ komplexer Brechungsindex

AFM Rasterkraftmikroskopie

SEM Rasterelektronenmikroskopie

TEM Transmissionselektronenmikroskopie 


\section{Dispersionen}

$\mathrm{SiO}_{2}$, Siliziumdioxid [Sopra database]

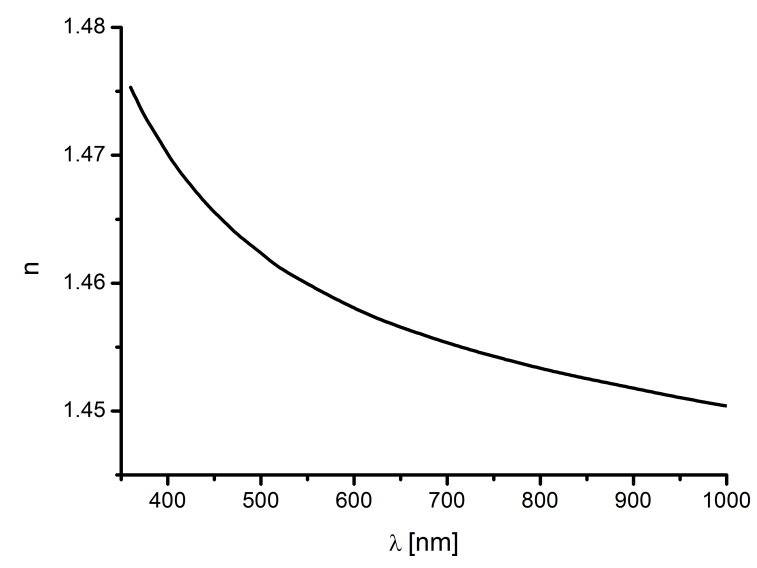

Abbildung 7.1 Dispersion $\mathrm{SiO}_{2}$

Si (100), Silizium [Sopra database]

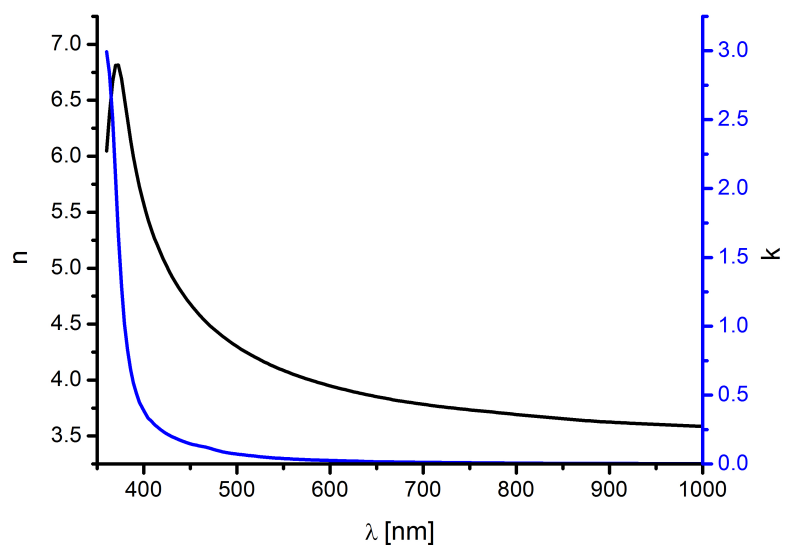

Abbildung 7.2 Dispersion Si (100)

PMMA, Polymethylmethacrylat [34] 


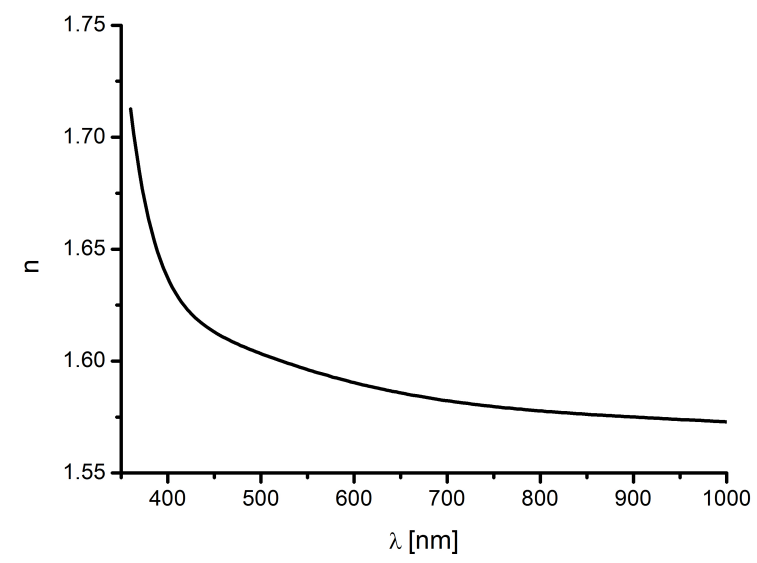

Abbildung 7.3 Dispersion PMMA

PS, Polystyrol [34]

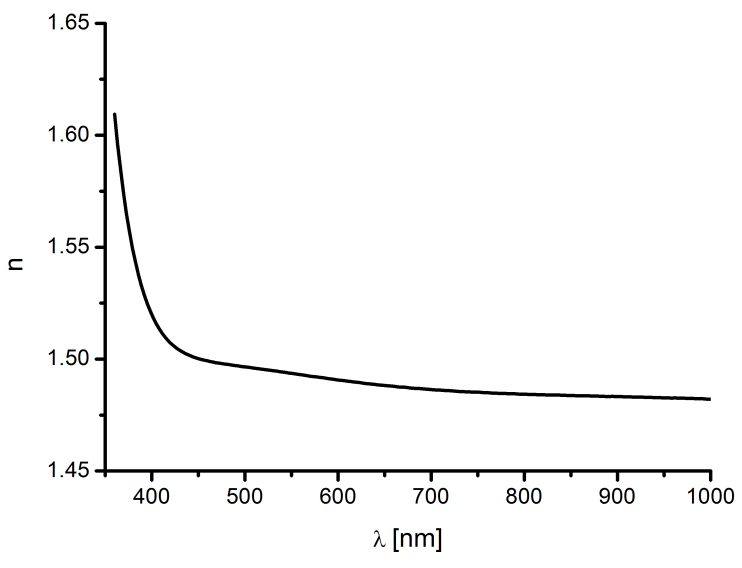

Abbildung 7.4 Dispersion PS

PET Polyethylenterephthalat, Cauchy-Model 


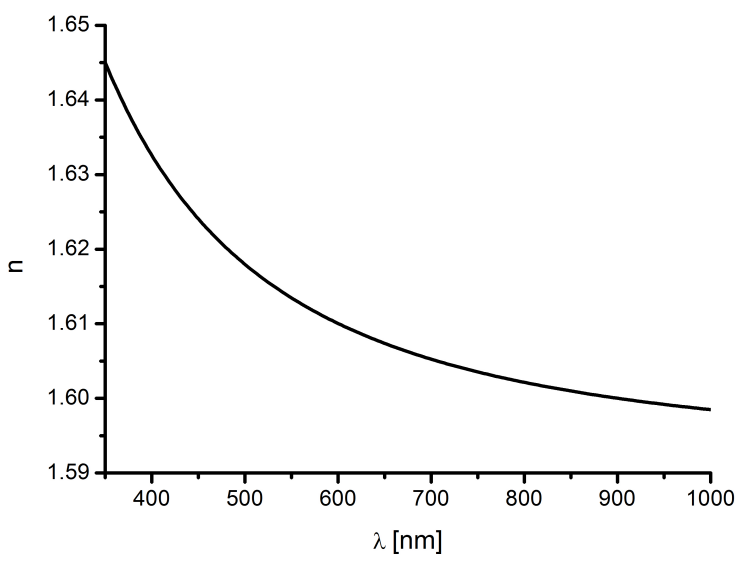

Abbildung 7.5 Dispersion PET (amorph) 


\section{Chemikalien}

PMMA, Sigam Aldrich, Mw $=120.000$<smiles>COC(=O)C(C)(C)CC(C)(C)C</smiles>

PS, Sigam Aldrich, $\mathrm{Mw}=280.000$<smiles>CC(C)(C)C(c1ccccc1)C(C)(C)C</smiles>

4-Cyano-4-[(dodecylsulfanylthiocarbonyl)sulfanyl]pentanoic acid, Sigma Aldrich<smiles>CCCCCCCCCCCCCCCCCCC(=O)O</smiles>

Polydimethylsiloxan (PDMS), Sylgard

Octadecyltrimethoxysilane, Sigma Aldrich

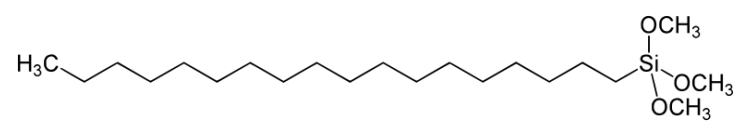

Wasserstoffperoxid (30 \%), Sigma Aldrich

Ammoniak (konz.), Sigma Aldrich

Toluol, Sigma Aldrich

Poly-3,4-ethylendioxythiophen-polystyrolsulfonat (PEDOT:PSS), Sigma Aldrich 
<smiles>CC(C)(C)c1sc(C(C)(C)C)c2c1OCCO2</smiles><smiles>CC(C)(C)CC(c1ccc(S(C)(=O)=O)cc1)C(C)(C)C</smiles>

Poly(3-hexylthiophen-2,5-diyl) (P3HT), Sigma Aldrich

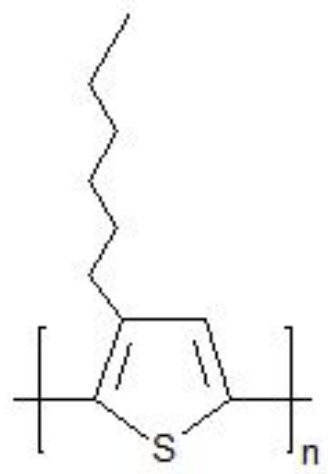

Polyethylenterephthalat (PET), Hostohan<smiles>COC(=O)c1ccc(C(=O)OCC(C)(C)C)cc1</smiles>

[6,6]-Phenyl-C61Buttersäuremethylester (PCBM)

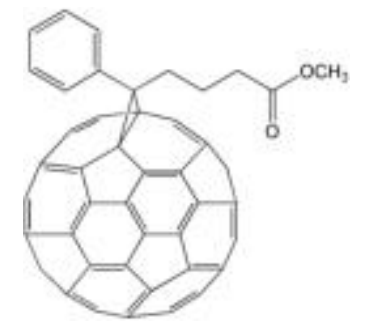

Aluminium-tris(8-hydroxychinolin) (AlQ3), Sigma Aldrich 


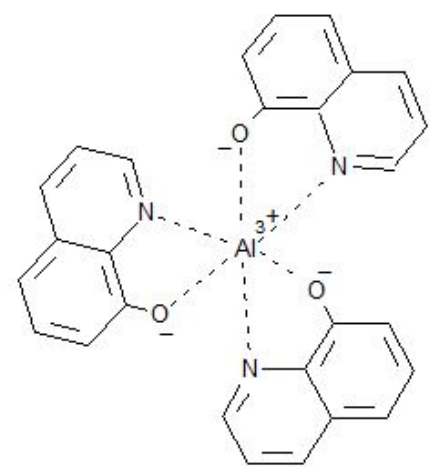

PEIE Polyethylenimin ethoxyliert

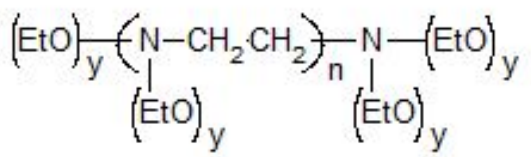

Poly[4,8-bis(5-(2-ethylhexyl)thiophen-2-yl)benzo[1,2-b;4,5-b']dithiophene-2,6-diyl-alt(4-(2-ethylhexyl)-3-fluorothieno[3,4-b] thiophene-)-2-carboxylate-2-6-diyl)] (PBDTTTEFT,PCE10) [64]<smiles></smiles>

Poly [[4,8-bis[(2-ethylhexyl)oxy]benzo[1,2-b:4,5-b']dithiophene-2,6-diyl][3-fluoro-2-[(2ethylhexyl)carbonyl]thieno[3,4-b]thiophenediyl]] (PTB7 ) [38]<smiles>CCCCCOc1c2cc(C(C)(C)C)sc2c(O)c2cc(-c3sc(F)c4c(F)c(C(=O)O)sc34)sc12</smiles>

Carotenoid-Porphyrin-Fulleren (CPF4) [63] 


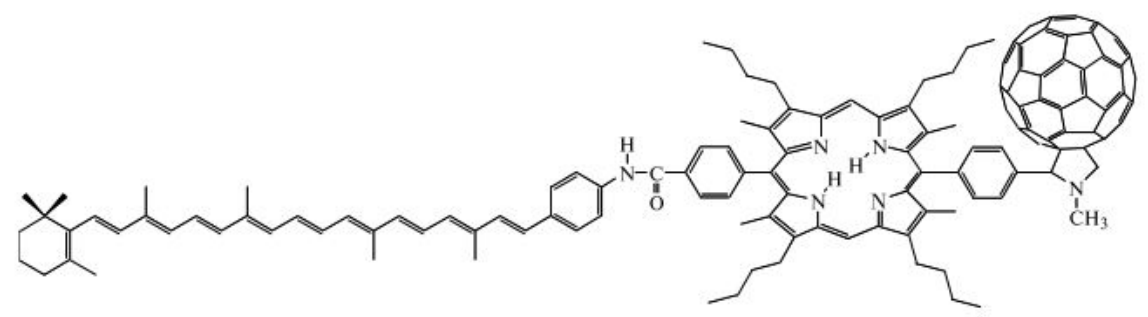

N2200 Poly\{[N,N-9bis(2-octyldodecyl)-naphthalene-1,4,5,8-bis(dicarboximide)-2,6-diyl]alt5,59-(2,29-bithiophene) $\},(\mathrm{P}(\mathrm{NDI} 2 \mathrm{OD}-\mathrm{T} 2))[44]$

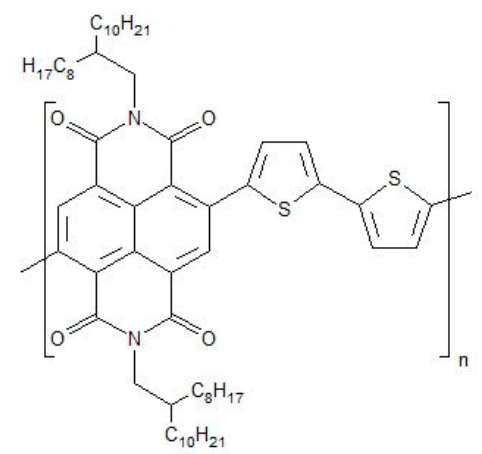

Poly-(3Z)-(7Z)-1,5-bis(2-octyldodecyl)-3,7-bis-[(5-thiophen-2-yl)-5,7-dihydro-1H,3Hpyrrolo[2,3-f]indole-2,6-dione-thiophene (DPIDT-2T) [48]

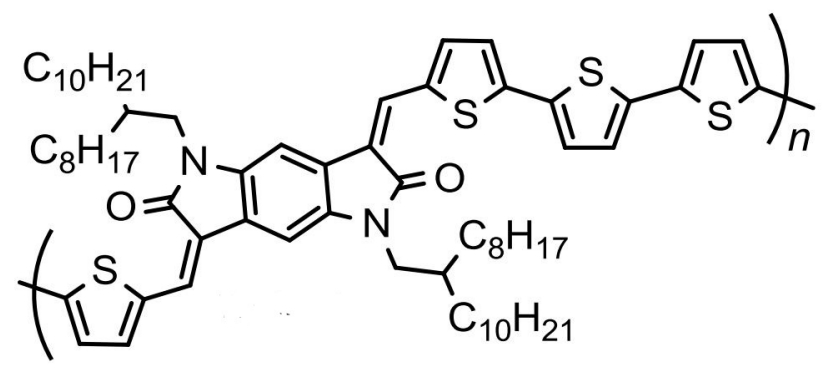




\section{Danksagung}

Mein Dank gilt meiner Familie und Freunden, die mich während der Bearbeitung meiner Dissertation unterstützt und an mich geglaubt haben. Besonders danken möchte ich Philipp Ahlborn und Ellen Rakete für die Unterstützung und vielen lieben Worte, während der Erarbeitung meiner Dissertation. Einen großen Dank an Prof. Philipp Vana für die Betreuung meiner Doktorarbeit. Der Accurion GmbH danke ich für die Möglichkeit zur Anfertigung der Dissertation. Des weiteren möchte ich mich bei T. Fricke-Begemann (LaserLaboratorium Göttingen e.V.), V. Bliznyuk (Clemson University), A.Meshalkin (Academy of Sciences of Moldova), E. Y. Poimanova (Donetsk National University), F. Bammer (TU Wien), A. Matkovic (University of Belgrade), J. W. Rumer (Imperial College London), C. MCNeill (Monash University) für die Kooperation und Unterstützung meiner wissenschaftlichen Arbeit durch Probensysteme bedanken. 


\section{Eidesstattliche Erklärung}

Hiermit erkläre ich an Eides statt, dass ich die vorliegende Arbeit selbstständig verfasst und keine als die angegebenen Quellen und Hilfsmittel verwendet habe. Die aus fremden Quellen direkt oder indirekt übernommenen Stellen sind als solche kenntlich gemacht.

Die Arbeit wurde bisher in gleicher oder ähnlicher Form keinem anderen Prüfungsamt vorgelegt und auch nicht veröffentlicht.

Göttingen, den 14. Juli 2017 